\title{
SPECTRAL INEQUALITY AND RESOLVENT ESTIMATE FOR THE BI-LAPLACE OPERATOR
}

\author{
JÉRÔME LE ROUSSEAU AND LUC ROBBIANO
}

\begin{abstract}
Aвstract. On a compact Riemannian manifold with boundary, we prove a spectral inequality for the bi-Laplace operator in the case of so-called "clamped" boundary conditions, that is, homogeneous Dirichlet and Neumann conditions simultaneously. We also prove a resolvent estimate for the generator of the damped plate semigroup associated with these boundary conditions. The spectral inequality allows one to observe finite sums of eigenfunctions for this fourth-order elliptic operator, from an arbitrary open subset of the manifold. Moreover, the constant that appears in the inequality grows as $\exp \left(C \mu^{1 / 4}\right)$ where $\mu$ is the largest eigenvalue associated with the eigenfunctions appearing in the sum. This type of inequality is known for the Laplace operator. As an application, we obtain a null-controllability result for a higher-order parabolic equation. The resolvent estimate provides the spectral behavior of the plate semigroup generator on the imaginary axis. This type of estimate is known in the case of the damped wave semigroup. As an application, we deduce a stabilization result for the damped plate equation, with a log-type decay.

The proofs of both the spectral inequality and the resolvent estimate are based on the derivation of different types of Carleman estimates for an elliptic operator related to the bi-Laplace operator: in the interior and at some boundaries. One of these estimates exhibits a loss of one full derivative. Its proof requires the introduction of an appropriate semi-classical calculus and a delicate microlocal argument.
\end{abstract}

KEYwORDs: high-order operators; boundary value problem; spectral inequality; resolvent estimate; interpolation inequality; controllability; stabilization; Carleman estimate; semiclassical calculus.

AMS 2010 SUBJeCT CLASSIFICATION: 35B45; 35J30; 35J40; 35K25; 35S15; 74K20; 93B05; 93B07; 93D15.

\section{Contents}

1. Introduction

1.1. On Carleman estimates

1.2. A method to prove the spectral inequality for the Laplace operator

1.3. Outline of the proof of the spectral inequality for the bi-Laplace operator 8

1.4. On Carleman estimates for higher-order elliptic operators 11

1.5. Some perspectives 12

1.6. Notation 13

1.7. Some basic properties of the bi-Laplace operator 13

Date: October 5, 2018.

Part of this article was written when both authors were on a leave at Insitut Henri-Poincaré, Paris. They wish to thank the institute for its hospitality. Both authors acknowledge support from Agence Nationale de la Recherche (grant ANR-13-JS01-0006 - iproblems - Problèmes Inverses). The authors also wish to thank two anonymous referees who have contributed to improve the presentation of this article by their remarks. 
2. $\quad$ Estimate away from boundaries 14

2.1. Simple-characteristic property of second-order factors 14

2.2. Local Carleman estimates away from boundaries 15

3. Estimate at the boundary $\{s=0\} \quad 17$

3.1. Tangential semi-classical calculus and associated Sobolev norms 17

$\begin{array}{ll}\text { 3.2. Statement of the Carleman estimate } & 18\end{array}$

3.3. Sub-ellipticity property 18

3.4. Proof of the estimate at $\{s=0\} \quad 19$

4. Estimate at the boundary $\left(0, S_{0}\right) \times \partial \Omega \quad 20$

4.1. A semi-classical calculus with three parameters 20

4.2. Local setting and statement of the Carleman estimate 26

$\begin{array}{lll}4.3 . & \text { Root properties } & 27\end{array}$

4.4. Microlocal regions 30

4.5. Proof strategies in the three microlocal regions 32

4.6. Microlocal estimate in the region $\mathcal{E}_{-} \quad 33$

4.7. Microlocal estimate in the region $\mathcal{E}_{0} \backslash F \quad 34$

4.8. $\quad$ Microlocal estimate in the region $F \quad 38$

4.9. Proof of the Carleman estimate of Theorem 4.17 40

5. Spectral inequality and application 43

5.1. An interpolation inequality 43

5.2. Spectral inequality 50

5.3. A null-controllability result for a higher-order parabolic equation 51

6. Resolvent estimate and application 51

6.1. Resolvent estimate 51

6.2. Proof of Lemma 6.1 52

6.3. A stabilization result for the plate equation 53

Appendices 53

A. Proofs of some technical results 53

A.1. Proof of the estimate optimality in the case of symbol flatness 53

A.2. Proofs associated with the semi-classical calculus 56

B. Elliptic and sub-elliptic estimates at the boundary $\left(0, S_{0}\right) \times \partial \Omega \quad 60$

B.1. Roots with negative imaginary part: a perfect elliptic estimate 60

$\begin{array}{lll}\text { B.2. } & \text { Sub-ellipticity quantification } & 60\end{array}$

$\begin{array}{lll}\text { B.3. } & \text { Estimates for first-order factors } & 61\end{array}$

B.4. Estimate concatenations 64

B.5. An Estimate for $Q_{k}$

Conflict of interest: $\quad 72$

$\begin{array}{ll}\text { References } & 72\end{array}$ 


\section{INTRODUCTION}

Let $A$ be the positive Laplace operator on a compact Riemannian manifold $(\Omega, \mathfrak{g})$, of dimension $d \geq 1$, with nonempty boundary $\partial \Omega$. In local coordinates, it reads

$$
A=-\Delta=|\mathfrak{g}|^{-1 / 2} \sum_{1 \leq i, j \leq d} D_{i}\left(|\mathfrak{g}|^{1 / 2} \mathfrak{g}^{i j} D_{j}\right),
$$

where $D=-i \partial$. For boundary conditions, say of homogeneous Dirichlet type ${ }^{1}$, we denote by $0<\omega_{1} \leq \cdots \leq \omega_{j} \leq \cdots$, the eigenvalues of the operator $A$, associated with a family $\left(\phi_{j}\right)_{j \in \mathbb{N}}$ of eigenfunctions that form a Hilbert basis for $L^{2}(\Omega)$. We refer to this selfadjoint operator as the Dirichlet Laplace operator. The following spectral inequality originates from [LR95, LZ98, JL99].

Theorem 1.1. Let $\mathscr{O}$ be an open subset of $\Omega$. There exists $C>0$ such that

$$
\|u\|_{L^{2}(\Omega)} \leq C e^{C \omega^{1 / 2}}\|u\|_{L^{2}(\mathscr{O})}, \quad \omega>0, \quad u \in \operatorname{Span}\left\{\phi_{j} ; \omega_{j} \leq \omega\right\}
$$

It provides an observation estimate of finite sums of eigenfunctions. The constant $C e^{C \omega^{1 / 2}}$ in the inequality is in fact optimal if $\mathscr{O} \Subset \Omega$ [JL99, LL12], and can be seen as a measure of the loss of orthogonality of the eigenfunctions $\phi_{j}$ when restricted to $\mathscr{O}$. This inequality has various applications. It can be used to prove the null-controllability of the heat equation [LR95] (see also the review article [LL12]), the null-controllability of the thermoelasticity system [LZ98], the null-controllability of the thermoelastic plate system [BN02, Mi107], and the null-controllability of some systems of parabolic PDEs [Léa10]. It can also be used to estimate the $(d-1)$-Hausdorff measure of the nodal set of finite sums of eigenfunctions of $A$, in the case of an analytic Riemannian manifold [JL99], recovering the result of [Lin91], that generalizes a result of [DF88] for eigenfunctions.

Consider now the unbounded operator acting on $H_{0}^{1}(\Omega) \times L^{2}(\Omega)$

$$
\mathcal{A}=\left(\begin{array}{cc}
0 & -1 \\
A & \alpha
\end{array}\right),
$$

with domain $D(\mathcal{A})=\left(H^{2}(\Omega) \cap H_{0}^{1}(\Omega)\right) \times H_{0}^{1}(\Omega)$, where $\alpha(x)$ is a nonnegative function. One can prove the following resolvent estimate [Leb96].

Theorem 1.2. Let $\mathscr{O}$ be an open subset of $\Omega$ and $\alpha$ be such that $\alpha(x) \geq \delta>0$ on $\mathscr{O}$. Then, the unbounded operator i $\sigma \mathrm{Id}-\mathcal{A}$ is invertible on $\mathscr{H}=H_{0}^{1}(\Omega) \times L^{2}(\Omega)$ for all $\sigma \in \mathbb{R}$ and there exist $K>0$ and $\sigma_{0}>0$ such that

$$
\left\|(i \sigma \mathrm{Id}-\mathcal{A})^{-1}\right\|_{\mathscr{L}(\mathscr{H}, \mathscr{H})} \leq K e^{K|\sigma|}, \quad \sigma \in \mathbb{R},|\sigma| \geq \sigma_{0} .
$$

This resolvent estimate allows one to deduce a logarithmic type stabilization result for the damped wave equation

$$
\partial_{t}^{2} y+A y+\alpha \partial_{t} y=0, \quad y_{\mid t=0}=y_{0}, \partial_{t} y_{\mid t=0}=y_{1}, \quad y_{\mid[0,+\infty) \times \partial \Omega}=0,
$$

for $y_{0}$ and $y_{1}$ chosen sufficiently regular, e.g. $\left(y_{0}, y_{1}\right) \in D(\mathcal{A})$ [Leb96, Bur98, BD08].

It is quite natural to wish to obtain similar inequalities for higher-order elliptic operators on $\Omega$, along with appropriate boundary conditions. The bi-Laplace operator, that can be encountered in models originating from elasticity for example, appears as a natural candidate for such a study. To understand some of the issues associated with the boundary conditions one may wish to impose let us consider the case of a spectral inequality of the form of (1.1). If the boundary conditions

\footnotetext{
${ }^{1}$ What we describe is yet valid for more general boundary conditions of Lopatinskii type for the Laplace operator.
} 
used for the bi-Laplace operator precisely make it the square of the Laplace operator $A$ (with its boundary conditions) then the spectral inequality is obvious as the eigenfunctions are the same for the two operators and $\lambda_{j} \geq 0$ is an eigenvalue of the bi-Laplace operator if and only if $\sqrt{\lambda_{j}}$ is one for the Laplace operator. To be clearer, let us consider the positive Dirichlet Laplace operator $A$. If $A^{2}$ is the bi-Laplace operator on $\Omega$ along with the boundary conditions $u_{\mid \partial \Omega}=0$ and $\Delta u_{\mid \partial \Omega}=0$, then the family $\left(\phi_{j}\right)_{j \in \mathbb{N}}$ introduced above, is in fact composed of eigenfunctions for $A^{2}$ associated with the eigenvalues $\lambda_{j}=\omega_{j}^{2}$. This set of boundary conditions is known as the "hinged" boundary conditions. We refer to this operator as the "hinged" bi-Laplace operator, and, for this operator, with Theorem 1.1, we directly have the following spectral inequality, for $\mathscr{O} \subset \Omega$,

$$
\|u\|_{L^{2}(\Omega)} \leq C e^{C \lambda^{1 / 4}}\|u\|_{L^{2}(\mathscr{O})}, \quad \lambda>0, \quad u \in \operatorname{Span}\left\{\phi_{j} ; \lambda_{j} \leq \lambda\right\} .
$$

One is naturally inclined to consider another set of boundary conditions, the so-called "clamped" boundary conditions, $u_{\mid \partial \Omega}=0$ and $\partial_{\nu} u_{\mid \partial \Omega}=0$, where $v$ is the outward normal to $\partial \Omega$. We refer to this operator as the "clamped" bi-Laplace operator. It is sometimes referred to as the DirichletNeumann bi-Laplace operator. Eigenfunctions of the "clamped" bi-Laplace operator are not related to eigenfunctions of the Dirichlet Laplace operator. In fact, observe that an eigenfunction of the "clamped" bi-Laplace operator cannot be an eigenfunction for the Laplace operator $A$, independently of the boundary conditions used for $A$. Indeed, from unique continuation arguments, if a $H^{2}$-function $\phi$ is such that $A \phi=\lambda \phi$ on $\Omega$ and $\phi_{\mid \partial \Omega}=\partial_{\nu} \phi_{\mid \partial \Omega}=0$, then $\phi$ vanishes identically. Thus, a spectral inequality for the "clamped" bi-Laplace cannot be deduced from a similar inequality for the Laplace operator $A$ with some well chosen boundary conditions. Yet, such an inequality is valuable to have at hand, in particular as the "clamped" bi-Laplace operator appears naturally in models. It is however often disregarded in the mathematical literature and replaced by the "hinged" bi-Laplace operator for which analysis can be more direct, in particular for the reasons we put forward above. A resolvent estimate of the form of (1.2) is also of interest towards stabilization results.

The main purpose of the present article is to show that a spectral inequality of the form of (1.1) and a resolvent estimate of the form (1.2) hold for the "clamped" bi-Laplace operator and, more generally, to provide some analysis tools to carefully study fourth-order operators that have a product structure. Carleman estimates will be central in the analysis here and we shall show how their derivation is feasible when the so-called sub-ellipticity condition does not hold, which is typical for product operators. If $B$ is the "clamped" bi-Laplace operator, that is, the unbounded operator $B=\Delta^{2}$ on $L^{2}(\Omega)$, with domain $D(B)=H^{4}(\Omega) \cap H_{0}^{2}(\Omega)$, which turn $B$ into a selfadjoint operator, let $\left(\varphi_{j}\right)_{j \in \mathbb{N}}$ be a family of eigenfunctions of $B$ that form a Hilbert basis for $L^{2}(\Omega)$, associated with the eigenvalues $0<\mu_{1} \leq \cdots \leq \mu_{j} \leq \cdots$ (the selfadjointness of $B$ and the existence of such a family are recalled in Section 1.7 below). We shall prove the following spectral inequality.

Theorem 1.3 (Spectral inequality for the "clamped" bi-Laplace operator). Let $\mathscr{O}$ be an open subset of $\Omega$. There exists $C>0$ such that

$$
\|u\|_{L^{2}(\Omega)} \leq C e^{C \mu^{1 / 4}}\|u\|_{L^{2}(\mathscr{O})}, \quad \mu>0, \quad u \in \operatorname{Span}\left\{\varphi_{j} ; \mu_{j} \leq \mu\right\} .
$$

Note that the spectral inequality of Theorem 1.3 was recently proven in [AE13] and [Gao16]. In [AE13] the coefficients and the domain are assumed to be analytic (the techniques used for the proof are then very different and exploit the analytic properties of the eigenfunctions). In [Gao16], the result is obtained in one space dimension; yet, therein, the factor $e^{C \mu^{1 / 4}}$ is replaced by $e^{C \mu^{1 / 2}}$, yielding a weaker form of the spectral inequality.

We shall present a null controllability result for the parabolic equation associated with $B$ which is a consequence of this spectral inequality. Such a result can be found in [AE13, EMZ15] in the case of analytic coefficients and domain. Here, coefficients are only assumed smooth. We 
conjecture that regularity could be lowered as low as $W^{1, \infty}$ for the coefficients in the principal part of the operator. This would require further developments in the line of what is done in [DCFL $\left.{ }^{+} 17\right]$ for instance. This would however significantly increase the size of the present article. Note that the analytic setting allows the authors of [AE13, EMZ15, EMZ17] to obtain control properties by only requiring the control domain to be of positive measure.

We shall also prove a resolvent estimate for the unbounded operator acting on $H_{0}^{2}(\Omega) \times L^{2}(\Omega)$,

$$
\mathcal{B}=\left(\begin{array}{cc}
0 & -1 \\
B & \alpha
\end{array}\right),
$$

with domain $D(\mathcal{B})=\left(H^{4}(\Omega) \cap H_{0}^{2}(\Omega)\right) \times H_{0}^{2}(\Omega)$, where $\alpha(x)$ is a nonnegative function.

Theorem 1.4. Let $\mathscr{O}$ be an open subset of $\Omega$ and $\alpha$ be such that $\alpha(x) \geq \delta>0$ on $\mathscr{O}$. Then, the unbounded operator i $\sigma \mathrm{Id}-\mathcal{B}$ is invertible on $\mathscr{H}=H_{0}^{2}(\Omega) \times L^{2}(\Omega)$ for all $\sigma \in \mathbb{R}$ and there exist $K>0$ and $\sigma_{0}>0$ such that

$$
\left\|(i \sigma \mathrm{Id}-\mathcal{B})^{-1}\right\|_{\mathscr{L}(\mathscr{H}, \mathscr{H})} \leq K e^{K|\sigma|^{1 / 2}}, \quad \sigma \in \mathbb{R},|\sigma| \geq \sigma_{0} .
$$

We shall present a log type stabilization result that is a consequence of Theorem 1.4 for the following damped plate equation

$$
\partial_{t}^{2} y+\Delta^{2} y+\alpha \partial_{t} y=0, \quad y_{\mid t=0}=y_{0}, \partial_{t} y_{\mid t=0}=y_{1}, \quad y_{\mid[0,+\infty) \times \partial \Omega}=\partial_{v} y_{\mid[0,+\infty) \times \partial \Omega}=0 .
$$

Both the proofs of the spectral inequality and the resolvent estimate are based on Carleman estimates for the fourth-order operator $P=D_{s}^{4}+B$.

The subject of the present article is connected to that of unique continuation, in particular through the use of Carleman estimates. Moreover, the spectral inequality of Theorem 1.3 is a quantified version of the unique continuation property for finite sums of eigenfunctions. There is an extensive literature on unique continuation for differential operators; yet, positive results require assumptions on the operator or on the hypersurface across which unique continuation is pursued. For instance, a simple-root assumption is often made following the work of A. Calderón [Cal58] or the celebrated strong pseudo-convexity condition is assumed following the work of L. Hörmander [Hör58, Hör63]. For second-order elliptic operators (with smooth complex coefficients) these assumptions are fulfilled. However, for higher-order operators they may not be satisfied. Counterexamples for the non uniqueness of fourth-order and higher-order operators with smooth coefficients can be found in [Pli61] and [Hör75]. See also the monograph [Zui83] for manifold positive and negative results. The question of strong unique continuation is also of interest for higher-order operators; see for instance [AB80] for a positive result and [Ali80] for a large class of negative results. Note that the above literature concerns unique continuation properties away from boundaries. For the results of Theorems 1.3 and 1.4 the analysis we use mainly focuses on the neighborhood of the boundary of the open set $\Omega$. There are few results on unique continuation near a boundary. Under the strong pseudo-convexity condition the unique continuation property can be obtained, even for higher-order operators; see [Tat96] and [BL15]. For the operator $P=D_{s}^{4}+B$ that we consider here, the strong pseudo-convexity property fails to hold near the boundary and also away from it. General approaches as developed in [Tat96, BL15] cannot be used. This is one of the interests of the present article.

1.1. On Carleman estimates. Carleman estimates are weighted a priori inequalities for the solutions of a partial differential equation (PDE), where the weight is of exponential type. For a partial differential operator $Q$ away from boundaries, it takes the form:

$$
\left\|e^{\tau \varphi} w\right\|_{L^{2}} \lesssim\left\|e^{\tau \varphi} Q w\right\|_{L^{2}}, \quad w \in \mathscr{C}_{c}^{\infty}(\Omega), \tau \geq \tau_{0} .
$$


The exponential weight involves a parameter $\tau$ that can be taken as large as desired. Additional terms in the l.h.s., involving derivatives of $u$, can be obtained depending on the order of $Q$ and on the joint properties of $Q$ and $\varphi$. For instance for a second-order operator $Q$, such an estimate can take the form

$$
\tau^{3 / 2}\left\|e^{\tau \varphi} u\right\|_{L^{2}}+\tau^{1 / 2}\left\|e^{\tau \varphi} D_{x} u\right\|_{L^{2}} \lesssim\left\|e^{\tau \varphi} Q u\right\|_{L^{2}}, \quad \tau \geq \tau_{0}, u \in \mathscr{C}_{c}^{\infty}(\Omega)
$$

One says that this estimate is characterized by the loss of a half derivative. This terminology originates from the underlying semi-classical calculus where one gives the same strengths to the parameter $\tau$ and to $D$. Whereas $Q$ is a second-order operator, the l.h.s. only exhibits derivatives or powers of $\tau$ of order 3/2. For most operators, this cannot be improved [Hör63, Hör85a]. In the proof of a Carleman estimate one introduces the so-called conjugated operator $Q_{\varphi}=e^{\tau \varphi} Q e^{-\tau \varphi}$, and estimate (1.5) reads

$$
\tau^{3 / 2}\|v\|_{L^{2}}+\tau^{1 / 2}\left\|D_{x} v\right\|_{L^{2}} \lesssim\left\|Q_{\varphi} v\right\|_{L^{2}}, \quad \tau \geq \tau_{0}, v=e^{\tau \varphi} u, \quad u \in \mathscr{C}_{c}^{\infty}(\Omega) .
$$

This type of estimate was used for the first time by T. Carleman [Car39] to achieve uniqueness properties for the Cauchy problem of an elliptic operator. Later, A.-P. Calderón and L. Hörmander further developed Carleman's method [Cal58, Hör58]. To this day, the method based on Carleman estimates remains essential to prove unique continuation properties; see for instance [Zui83] for an overview. On such questions, more recent advances have been concerned with differential operators with singular potentials, starting with the contribution of D. Jerison and C. Kenig [JK85]. There, Carleman estimates rely on $L^{p}$-norms rather than $L^{2}$-norms as in the estimates above. The proof of such $L^{p}$ Carleman estimates is very delicate. The reader is also referred to [Sog89, KT01, KT02, DSF05, KT05]. In more recent years, the field of applications of Carleman estimates has gone beyond the original domain; they are also used in the study of:

- Inverse problems, where Carleman estimates are used to obtain stability estimates for the unknown sought quantity (e.g. coefficient, source term) with respect to norms on measurements performed on the solution of the PDE, see e.g. [BK81, Isa98, Kub00, IIY03]; Carleman estimates are also fundamental in the construction of complex geometrical optic solutions that lead to the resolution of inverse problems such as the Calderón problem with partial data [KSU07, DSFKSU09].

- Control theory for PDEs; Carleman estimates yield the null controllability of linear parabolic equations [LR95] and the null controllability of classes of semi-linear parabolic equations [FI96, Bar00, FCZ00]. They can also be used to prove unique continuation properties, that in turn are crucial for the treatment of low frequencies for exact controllability results for hyperbolic equations as in [BLR92].

To indicate how the spectral inequality of Theorem 1.3 for the bi-Laplace operator $B$ can be proven by means of Carleman estimates, we first review a method, that yields the spectral inequality of Theorem 1.1 for the Laplace operator $A$. In this introductory section, we have chosen to mainly focus on the method of proof of the spectral inequality; a comprehensive presentation including a presentation of the proof of the resolvent estimates of Theorems 1.2 and 1.4 would not bring any further insight to the reader as the line of arguments is quite similar.

1.2. A method to prove the spectral inequality for the Laplace operator. The method we describe here originates from [LR95]. We consider the elliptic operator $P_{A}=D_{s}^{2}+A$ on $Z=$ $\left(0, S_{0}\right) \times \Omega$, for some $S_{0}>0$ meant to remain fixed. We also pick $0<\alpha<S_{0} / 2$. Three different types of Carleman estimates are proven for the operator $P_{A}$ : (i) in the interior of $\left(0, S_{0}\right) \times \Omega$; (ii) at the boundary $\{s=0\} \times \Omega$; (iii) at the boundary $\left(\alpha, S_{0}-\alpha\right) \times \partial \Omega$. The three regions where these Carleman estimates are derived are illustrated in Figure 1. It is simpler to first describe Case (i), 


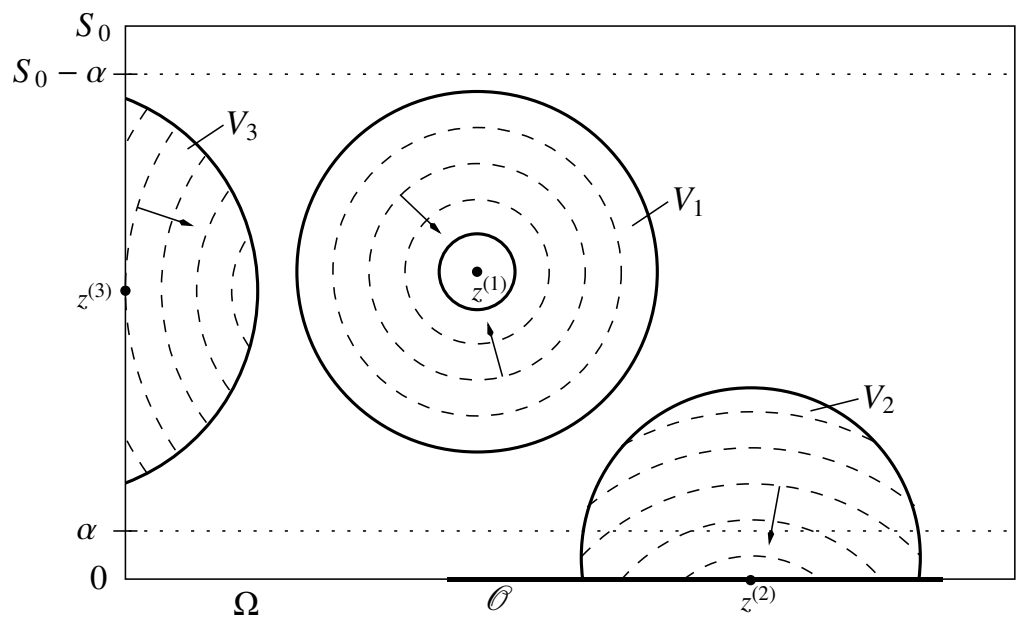

FIGURE 1. Location and geometry of the three types of estimates. Dashed are level sets for the weight functions $\varphi$ used in the Carleman estimates. Arrows represent the directions of the (non vanishing) gradient of $\varphi$.

that is, the estimate in the interior. In Figure 1, this corresponds to the neighborhood $V_{1}$ of some point $z^{(1)} \in Z$. There, the Carleman estimate for this operator $P_{A}$ is of the form described above, that is,

$$
\tau^{3 / 2}\left\|e^{\tau \varphi} w\right\|_{L^{2}(Z)}+\tau^{1 / 2}\left\|e^{\tau \varphi} D_{z} w\right\|_{L^{2}(Z)} \lesssim\left\|e^{\tau \varphi} P_{A} w\right\|_{L^{2}(Z)},
$$

where the weight function $\varphi=\varphi(z)$ is real-valued with a non-vanishing gradient, $\tau$ is a large positive parameter, and $w$ is any smooth function compactly supported in $V_{1}$. In fact, this estimate holds if the so-called sub-ellipticity condition is fulfilled by $P_{A}$ and $\varphi$. If $p_{A}(z, \zeta)$ is the principal symbol of $P_{A}$, the sub-ellipticity condition in $V_{1}$ reads

$$
p_{A}(z, \zeta+i \tau d \varphi(z))=0 \Rightarrow \frac{1}{2 i}\left\{\overline{p_{A}(z, \zeta+i \tau d \varphi(z))}, p_{A}(z, \zeta+i \tau d \varphi(z))\right\}>0,
$$

for $z \in V_{1}, \zeta \in \mathbb{R}^{d+1}$, and $\tau \geq 0$. It is in fact equivalent to a Carleman estimate of the form (1.6) for $P_{A}$ (see [Hör63] or [LL12]). Observe that $p_{A}(z, \zeta+i \tau d \varphi(z))$ is the semi-classical principal symbol of the conjugated operator $P_{A, \varphi}=e^{\tau \varphi} P_{A} e^{-\tau \varphi}$.

The function $\varphi$ is chosen of the form $\varphi(z)=\exp \left(-\gamma\left|z-z^{(1)}\right|^{2}\right)$ and $V_{1}$ is an annulus around $z^{(1)}$, thus avoiding where the gradient of $\varphi$ vanishes (see Figure 1). For $\gamma>0$ chosen sufficiently large, one can prove that the sub-ellipticity condition (1.7) holds and thus estimate (1.6) is achieved (see e.g. [LR95] or [LL12]).

From estimate (1.6), one can deduce the following local interpolation inequality, for all $r>0$ chosen sufficiently small, for some $\delta \in(0,1)$ (see e.g. [LR95]),

$$
\|v\|_{H^{1}\left(B\left(z^{(1)}, 3 r\right)\right)} \lesssim\|v\|_{H^{1}(Z)}^{1-\delta}\left(\left\|P_{A} v\right\|_{L^{2}(Z)}+\|v\|_{H^{1}\left(B\left(z^{(1)}, r\right)\right)}\right)^{\delta}, \quad v \in H^{2}(Z) .
$$

We now consider Case (ii). In a neighborhood $V_{2}$ of a point $z^{(2)} \in\{0\} \times \mathscr{O}$, one can derive an estimate of the same form as (1.6), yet, with two trace terms in the r.h.s., that is,

$$
\sum_{|\beta| \leq 1} \tau^{3 / 2-|\beta|}\left\|e^{\tau \varphi} D^{\beta} w\right\|_{L^{2}(Z)} \lesssim\left\|e^{\tau \varphi} P_{A} w\right\|_{L^{2}(Z)}+\tau^{1 / 2}\left(\left|e^{\tau \varphi} w_{\mid s=0^{+}}\right|_{H^{1}(\mathscr{O})}+\left|e^{\tau \varphi} \partial_{s} w_{\mid s=0^{+}}\right|_{L^{2}(\mathscr{O})}\right),
$$


for $\tau \geq \tau_{0} \geq 1$ and $w$ smooth up to the boundary $\left\{s=0\right.$, with $\operatorname{supp}(w) \cap Z \subset V_{2}$, with $V_{2}$ as represented in Figure 1. This can be obtained by locally choosing a weight function of the form $\varphi(z)=\exp (\gamma \psi(z))$ with $\psi(z)$ such that $\partial_{s} \psi(z) \leq-C<0$ in $V_{2}$ and choosing the parameter $\gamma>0$ sufficiently large (see e.g. [LZ98]). We use the notation \|..\| for functions in the interior of the domain and |.| for functions on the boundaries.

From estimate (1.9) one deduces the following local interpolation inequality: there exist $V \subset V_{2}$ and $\delta \in(0,1)$ such that

$$
\|v\|_{H^{1}(V \cap Z)} \lesssim\|v\|_{H^{1}(Z)}^{1-\delta}\left(\left\|P_{A} v\right\|_{L^{2}(Z)}+\left|v_{\mid s=0^{+}}\right|_{H^{1}(\mathscr{O})}+\left|\partial_{s} v_{\mid s=0^{+}}\right|_{L^{2}(\mathscr{O})}\right)^{\delta}, \quad v \in H^{2}(Z) .
$$

We finally consider Case (iii). In a neighborhood of a point $z^{(3)} \in\left(\alpha, S_{0}-\alpha\right) \times \partial \Omega$, one can derive an estimate of the same form as (1.6), yet, with a single trace term in the r.h.s., that is,

$$
\begin{aligned}
\left.\sum_{|\beta| \leq 1} \tau^{3 / 2-|\beta|}\left|\left\|e^{\tau \varphi} D^{\beta} w\right\|_{L^{2}(Z)}+\tau^{1 / 2}\right| e^{\tau \varphi} \partial_{\nu} w_{\mid \partial Z}\right|_{L^{2}\left(\left(\alpha, S_{0}-\alpha\right) \times \partial \Omega\right)} \\
\\
\quad \lesssim\left\|e^{\tau \varphi} P_{A} w\right\|_{L^{2}(Z)}+\sum_{\left|\beta^{\prime}\right| \leq 1} \tau^{3 / 2-\left|\beta^{\prime}\right|}\left|e^{\tau \varphi} D_{\top}^{\beta^{\prime}} w_{\mid \partial Z}\right|_{L^{2}\left(\left(\alpha, S_{0}-\alpha\right) \times \partial \Omega\right)},
\end{aligned}
$$

for $\tau \geq \tau_{0} \geq 1$ and $w$ smooth up to the boundary $\left(\alpha, S_{0}-\alpha\right) \times \partial \Omega$, with $\operatorname{supp}(w) \cap Z \subset V_{3}$, with $V_{3}$ as represented in Figure 1. This can be obtained by locally choosing a weight function of the form $\varphi(z)=\exp (\gamma \psi(z))$ with $\psi(z)$ such that $\partial_{\nu} \psi(z) \leq-C<0$ in $V_{3}$, where $v$ is the outward normal to $\partial \Omega$, and choosing the parameter $\gamma>0$ sufficiently large (see e.g. [LR95]). Here, for $\left|\beta^{\prime}\right| \geq 1$, $D_{\mathrm{T}}^{\beta^{\prime}}$ stand as differentiations in the tangential directions only, along vector fields that form a local frame.

From estimate (1.11) one deduces the following local interpolation inequality: there exist $V \subset$ $V_{3}$, with $\bar{V}$ neighborhood of $z^{(3)}$ in $\bar{Z}$, some open subset $\mathscr{Q} \subset V_{3}$ with positive distance to the boundary, and $\delta \in(0,1)$ such that

$$
\|v\|_{H^{1}(V \cap Z)} \lesssim\|v\|_{H^{1}(Z)}^{1-\delta}\left(\left\|P_{A} v\right\|_{L^{2}(Z)}+\|v\|_{H^{1}(\mathscr{Q})}\right)^{\delta}, \quad v \in H^{2}(Z), \quad v_{\left(0, S_{0}\right) \times \partial \Omega}=0 .
$$

The three interpolation inequalities (1.8), (1.10), and (1.12) can be used to form a global interpolation inequality, by means of compactness arguments. In particular, the interior inequality (1.8) permits the "propagation" of the estimate. Then, there exists $\delta \in(0,1)$, such that

$$
\|v\|_{H^{1}\left(\left(\alpha, S_{0}-\alpha\right) \times \Omega\right)} \lesssim\|v\|_{H^{1}(Z)}^{1-\delta}\left(\left\|P_{A} v\right\|_{L^{2}(Z)}+\left|v_{\mid s=0^{+}}\right|_{H^{1}(\mathscr{O})}+\left|\partial_{s} v_{\mid s=0^{+}}\right|_{L^{2}(\mathscr{O})}\right)^{\delta},
$$

for $v \in H^{2}(Z)$ satisfying $v_{\mid\left(0, S_{0}\right) \times \partial \Omega}=0$. This inequality then implies the spectral property for the Laplace operator for $u=\sum_{\omega_{j} \leq \omega} u_{j} \phi_{j} \in \operatorname{Span}\left\{\phi_{j} ; \omega_{j} \leq \omega\right\}$, if applied to a well chosen function $v(s, x)$, namely,

$$
v(s, x)=\sum_{\omega_{j} \leq \omega} u_{j} \omega_{j}^{-1 / 2} \sinh \left(\omega_{j}^{1 / 2} s\right) \phi_{j}(x) .
$$

Details can for instance be found in [LL12]. In the present paper, we shall apply this approach for the bi-Laplace operator, the argument is provided in details in Section 5.2.

1.3. Outline of the proof of the spectral inequality for the bi-Laplace operator. Above we described how Carleman estimates can be used to prove a spectral inequality of the form given in Theorem 1.1 for the Laplace operator. To prove the spectral inequality of Theorem 1.3 for the "clamped" bi-Laplace operator, we shall prove several Carleman estimates for the following fourth-order elliptic operator

$$
\left.P=D_{s}^{4}+\Delta^{2} \quad \text { on } Z=0, S_{0}\right) \times \Omega .
$$


As for $P_{A}$ above, we shall prove such estimates at three different locations: (i) in the interior of $\left(0, S_{0}\right) \times \Omega$, in Section 2; (ii) at the boundary $\{s=0\} \times \Omega$, in Section 3; (iii) at the boundary $\left(\alpha, S_{0}-\alpha\right) \times \partial \Omega$, in Section 4. In Section 5, these three types of estimations are then used to achieve local interpolation inequalities that can be used to prove, first, a global interpolation inequality and, second, the spectral inequality of Theorem 1.3. Note that for the proof of the resolvent estimate of Theorem 1.4 only steps (ii) and (iii) are needed.

Cases (i) and (ii). The weight functions that we shall use will be the same as that used for the operator $P_{A}$ for Cases (i) and (ii). In Case (ii), the estimate we obtain for $P$ takes the form

$$
\begin{aligned}
\tau^{-1 / 2} \sum_{|\alpha| \leq 4}\left\|\tau^{4-|\alpha|} e^{\tau \varphi} D_{s, x}^{\alpha} u\right\|_{L^{2}(Z)} \lesssim\left\|e^{\tau \varphi} P u\right\|_{L^{2}(Z)} & \\
& +\sum_{j=0}^{3}\left(\tau^{7 / 2-j}\left|e^{\tau \varphi} D_{s}^{j} u_{\mid s=0^{+}}\right|_{L^{2}(\Omega)}+\left|e^{\tau \varphi} D_{s}^{j} u_{\mid s=0^{+}}\right|_{H^{7 / 2-j}(\Omega)}\right),
\end{aligned}
$$

for functions localized near a point $z^{(2)} \in\{0\} \times \mathscr{O}$, with $\mathscr{O} \subset \Omega$. We have observation terms at the boundary $\{s=0\}$. We use the notation $\|$.$\| for functions in the interior of the domain and |.| for$ functions on the boundaries.

Note that this estimate is characterized by the loss of half-derivative, similarly to the estimate one can derive for $P_{A}$. In fact, the sub-ellipticity condition holds in $V_{2}$ despite the fact that $P_{\varphi}=$ $e^{\tau \varphi} P e^{-\tau \varphi}$ can be written as a product of two operators, $P_{\varphi}=Q_{1} Q_{2}$, as, here, $\operatorname{char}\left(Q_{1}\right) \cap \operatorname{char}\left(Q_{2}\right)=$ $\emptyset$.

In Case (i), however, the estimate we obtain is characterized by the loss of one full derivative, taking the form

$$
\sum_{|\alpha| \leq 4} \tau^{3-|\alpha|}\left\|e^{\tau \varphi} D^{\alpha} u\right\|_{L^{2}(Z)} \lesssim\left\|e^{\tau \varphi} P u\right\|_{L^{2}(Z)},
$$

for functions compactly supported away from boundaries. In fact, this loss cannot be improved as explained in Section 1.4. Here also, the operator $P_{\varphi}$ can be written as a product of two operators, $P_{\varphi}=Q_{1} Q_{2}$, and here, as opposed to Case (ii), we have $\operatorname{char}\left(Q_{1}\right) \cap \operatorname{char}\left(Q_{2}\right) \neq \emptyset$.

We provide fairly short proofs of the Carleman estimates in Cases (i) and (ii) in Sections 2 and 3. Note, however, that the loss of a full derivative in Case (i) does not create any obstruction to the derivation of a local interpolation inequality in Section 5.

Remark 1.5. Sub-ellipticity does not hold in $V_{1}$. The reader should note that the failure of the sub-ellipticity property does not automatically imply a loss of one full derivative. The phenomena that can occur require a fine analysis to be understood. This is carried out in [Ler88]. Roughly speaking, if sub-ellipticity does not hold, and if some iterated Poisson brackets vanish up to order $k$ and an iterated Poisson bracket of order $k+1$ is positive, then an estimate can be obtained with a loss of $k /(k+1)$ derivative. In the present case, as we can prove that the loss of one full derivative cannot be improved, we then know that all the iterated Poisson brackets used in [Ler88] vanish. The essential problem is that the conjugated operator $P_{\varphi}$ can be written as a product of two operators $Q_{1} Q_{2}$, and in the case $\operatorname{char}\left(Q_{1}\right) \cap \operatorname{char}\left(Q_{2}\right) \neq \emptyset$, not only does sub-ellipticity not hold, but we see that the iterated Poisson brackets also vanish.

Case (iii). This case is delicate and the derivation of the Carleman estimate at the boundary $\left(\alpha, S_{0^{-}}\right.$ $\alpha) \times \partial \Omega$ is one of the main results of the present article. This case is also precisely where we have to take into account the boundary conditions for the bi-Laplace operator $B$. The estimate we obtain in Case (iii) in Section 4 is characterized by the loss of one full derivative and, as for case (i), this cannot be improved as explained in Section 1.4. This is a source of major complications for the proof of the Carleman estimate itself. As in Case (i) this, however, does not create any obstruction 
in the derivation of the local interpolation inequality in Section 5. In fact, the proof of the local Carleman estimate in $V_{3}$, a neighborhood of a point of the boundary $\left(\alpha, S_{0}-\alpha\right) \times \partial \Omega$, requires microlocal arguments. This implies the introduction of microlocalization operators that realize some partition of unity in phase space over $V_{3}$. For each induced microlocal region, a Carleman estimate is derived. One region is less favorable: there, the fourth-order conjugated operator $P_{\varphi}$ can we written as a product of four first-order factors, and two of them fail to be elliptic. Moreover, their characteristic sets intersect; sub-ellipticity does not hold there and, in fact, this generates a loss of a full derivative in the estimation. There, the a priori estimate one derives permits to only estimate the semi-classical $H^{3}$-norm, viz., $\|w\|_{3, \tau} \asymp \tau^{3}\|w\|_{L^{2}}+\|w\|_{H^{3}}$. In other microlocal regions over $V_{3}$, the conjugated operator $P_{\varphi}$ exhibits at most a non elliptic first-order factor only yielding a half derivative loss as sub-ellipticity holds. If one does not proceed carefully, the derivation in the least favorable region yields error terms that can be of the same strength as the norm $\|w\|_{3, \tau}$, preventing to conclude positively to the Carleman estimate.

We define the weight function in the form $\varphi(z)=e^{\gamma \psi(z)}$ and keep track of the parameter $\gamma$ that is meant to be large. The function $\psi$ is chosen such that $\partial_{\nu} \psi \leq-C<0$ in a neighborhood of a point of the boundary where we try to derive the Carleman estimate. The use of an exponential form for the weight function can already be found in the seminal work of L. Hörmander ([Hör63, Section 8.6] and [Hör85a, Section 28.3]), in connexion with the celebrated notions of pseudo-convexity and strong pseudo-convexity. This introduces a second large parameter. Several authors have derived Carleman estimates for some operators in which the dependence upon the second large parameters is explicit. See for instance [FI96]. Such result can be very useful to address applications such as inverse problems. On such questions see for instance [El100, EI00, IK08, BY12]. In [Le 15], an analysis framework is introduced, based on the Weyl-Hörmander calculus ([Hör79], [Hör85b, Sections 18.4-18.6]), that allows one to describe the explicit dependence of Carleman upon the second large parameter $\gamma$ for general classes of operators. That analysis is carried out away from boundaries. Here, we use that approach by means of a tangential Weyl-Hörmander calculus. The introduction of the second large parameter $\gamma$ allows us to handle some error terms in the derivation of the Carleman estimate in $V_{3}$. This is however not sufficient to have control over all the error terms that appear in the microlocal region within $V_{3}$ where sub-ellipticity does not hold, since the operator under study is a product of two second-order operators (see above).

Yet, when one attempts to derive the estimate, one realizes that the derivation is possible in the case $\varphi$, and thus $\psi$, only depend on the normal variable to the boundary. Yet, for the interpolation inequality we wish to derive at the boundary $\left(\alpha, S_{0}-\alpha\right) \times \partial \Omega$, some convexity of the level sets of the weight function $\varphi$ is needed: $\varphi$ cannot be constant along the boundary. This is illustrated in Figure 1 (in the neighborhood $V_{3}$ ). We thus introduce the function $\psi_{\varepsilon}(z)=\psi\left(\varepsilon z^{\prime}, z_{N}\right)$, where $z^{\prime}$ denotes the tangential variables and $z_{N}$ denotes the normal variable (in local coordinates where the boundary is given by $\left.\left\{z_{N}=0\right\}\right)$, and we set $\varphi(z)=e^{\gamma \psi_{\varepsilon}(z)}$. Here, $\varepsilon$ is a small parameter, $\varepsilon \in(0,1)$. Keeping track of the dependence of the microlocal estimates in this third parameter too then allows one to obtain a Carleman estimate, at the boundary, with a weight function with some convexity of its level sets with respect to the boundary. This is precisely done by extending some of the work of [Le 15] and introducing a Weyl-Hörmander calculus, with three parameters: the large semiclassical parameter $\tau$, the second large parameter $\gamma$, and this new parameter $\varepsilon \in(0,1)$ that controls the convexity of the level sets of the weight function. Note that even in the case $\psi=\psi\left(z_{n}\right)$, the proof of the Carleman estimate relies on taking the second parameter $\gamma$ sufficiently large (see the end of Proposition 4.25 below). The introduction of the parameter $\varepsilon$ alone would not be sufficient. Only the joint introduction of the two parameters allows us to conclude positively to the Carleman estimate in the microlocal region where a full derivative is lost. 
All the different microlocal estimates need to be derived within the refined semi-classical calculus with three parameters. Arguments are based on the ellipticity or sub-ellipticity of the different factors building the fourth-order operator $P_{\varphi}$, and the position of theirs roots in the complex plane. This analysis follows in part from the different works [Be103, LR10, LR11, LL13, CR14].

Eventually, the various microlocal estimates we obtain need to be patched together. This procedure generates commutators of the fourth operator $P_{\varphi}$ and the microlocal cut-offs, leading to some third-order error terms that can be handled thanks to the better microlocal estimates obtained away from the least favorable region.

Near a point of the boundary $\partial Z=\left(0, S_{0}\right) \times \partial \Omega$ locally written in the form $\left\{x_{d}=0\right\}$ with $Z=\left(0, S_{0}\right) \times \Omega=\left\{x_{d}>0\right\}$, the estimate we obtain, for $\tau$ and $\gamma$ large and $\varepsilon$ small, is of the form

$$
\gamma \sum_{|\alpha| \leq 4}\left\|\tilde{\tau}^{3-|\alpha|} e^{\tau \varphi} D_{s, x}^{\alpha} u\right\|_{L^{2}(Z)}+\sum_{0 \leq j \leq 3}\left|e^{\tau \varphi} D_{x_{d}}^{r} u_{\mid \partial Z}\right|_{7 / 2-j, \tilde{\tau}} \lesssim\left\|e^{\tau \varphi} P u\right\|_{L^{2}(Z)}+\sum_{j=0,1}\left|e^{\tau \varphi} D_{x_{d}}^{j} u_{\mid \partial Z}\right|_{7 / 2-j, \tilde{\tau}} .
$$

On the 1.h.s. we find norms of all traces; on the r.h.s. we only have observation with the traces $u_{\mid \partial Z}$ and $D_{x_{d}} u_{\mid \partial Z}$ associated with the clamped boundary conditions. Here $\tilde{\tau}=\tau \gamma \varphi$.

1.4. On Carleman estimates for higher-order elliptic operators. If $Q$ is an elliptic operator of even order $m$, and $\varphi$ is a weight function such that the couple $(P, \varphi)$ satisfies the sub-ellipticity condition (as stated above), then a Carleman estimate can be obtained, even at a boundary, for instance with the results of [BL15]. We use those results in Section 3 for the proof of the Carleman estimate at the boundary $\{s=0\}$.

If $m \geq 4$, it is however quite natural to not have the sub-ellipticity condition, in particular if the operator $Q$ is in the form of a product of two operators, say $Q=Q_{1} Q_{2}$. Denote by $q, q_{1}$, and $q_{2}$ the principal symbols of $Q, Q_{1}$, and $Q_{2}$ respectively. The conjugated operator $Q_{\varphi}=e^{\tau \varphi} Q e^{-\tau \varphi}$ reads $Q_{\varphi}=Q_{1, \varphi} Q_{2, \varphi}$, with $Q_{k, \varphi}=e^{\tau \varphi} Q_{k} e^{-\tau \varphi}, k=1,2$. If we have $\operatorname{char}\left(Q_{1, \varphi}\right) \cap \operatorname{char}\left(Q_{2, \varphi}\right) \neq \emptyset$ then the sub-ellipticity condition fails to hold. In fact, if $q_{\varphi}, q_{1, \varphi}$, and $q_{2, \varphi}$ are the semi-classical principal symbols of $Q_{\varphi}, Q_{1, \varphi}$, and $Q_{2, \varphi}$, that is, $q_{\varphi}=q(z, \zeta+i \tau d \varphi(z))$ and $q_{k, \varphi}=q_{k}(z, \zeta+i \tau d \varphi(z)), k=1,2$, we can write

$$
\left\{\bar{q}_{\varphi}, q_{\varphi}\right\}=\left|q_{1, \varphi}\right|^{2}\left\{\bar{q}_{2, \varphi}, q_{2, \varphi}\right\}+\left|q_{2, \varphi}\right|^{2}\left\{\bar{q}_{1, \varphi}, q_{1, \varphi}\right\}+f\left|q_{1, \varphi}\right|\left|q_{2, \varphi}\right|,
$$

for some function $f$. Thus $\left\{\bar{q}_{\varphi}, q_{\varphi}\right\}$ vanishes if $q_{1, \varphi}=q_{2, \varphi}=0$. Then, the sub-ellipticity property of (1.7) cannot hold for $Q$.

Observe that in the above example we have $d_{z, \zeta} q(z, \zeta+i \tau d \varphi(z))=0$ if $q_{2}(z, \zeta+i \tau d \varphi(z))=$ $q_{1}(z, \zeta+i \tau d \varphi(z))=0$. The following proposition (that applies to operators that need not be elliptic) shows that in such case of symbol "flatness", the Carleman estimate we can derive for $Q$ exhibits at least a loss of one full derivative.

Proposition 1.6. Let $Q=Q\left(z, D_{z}\right)$ be a smooth operator of order $m \geq 1$ in $Z$, an open subset of $\mathbb{R}^{N}$. Assume further that there exist a smooth weight function $\varphi(z), C>0, \tau_{1}>0$, a multi-index $\alpha$ with $0 \leq|\alpha| \leq m$, and $\delta \geq 0$ such that

$$
\tau^{m-1-|\alpha|+\delta}\left\|e^{\tau \varphi} D_{z}^{\alpha} u\right\|_{L^{2}} \leq C\left\|e^{\tau \varphi} Q u\right\|_{L^{2}}
$$

for $\tau \geq \tau_{1}$ and for $u \in \mathscr{C}^{\infty}\left(\mathbb{R}^{N}\right)$ with $\operatorname{supp}(u) \subset Z$. Let $q(z, \zeta)$ be the principal symbol of $Q$. If there exist $z_{0} \in Z, \zeta_{0} \in \mathbb{R}^{N}$ and $\tau_{0}>0$ such that $\theta_{0}^{\alpha} \neq 0$, with $\theta_{0}=\zeta_{0}+i \tau_{0} d \varphi\left(z_{0}\right)$, and

$$
q\left(z_{0}, \theta_{0}\right)=q_{\varphi}\left(z_{0}, \zeta_{0}, \tau_{0}\right)=0, \quad d_{z, \zeta} q\left(z_{0}, \theta_{0}\right)=0,
$$

then $\delta=0$.

In other words, if there is a point $\left(x_{0}, \xi_{0}, \tau_{0}\right)$ where the symbol $q_{\varphi}$ vanishes at second order, then if a Carleman estimate holds it exhibits at least the loss of a full derivative.

We refer to Section A.1 for a proof. 
Remark 1.7. This loss of at least one full derivative shows that the analysis of [Ler88] cannot be applied here, as it concerns Carleman estimate with losses of less that one derivative. In particular, one can check that iterated Poisson brackets used in [Ler88] all vanish at points where $q_{\varphi}$ vanishes at second order.

In dimension greater than 1 , this proposition applies to the bi-Laplace operator $B$ introduced above on the manifold $\Omega$. If $a(x, \xi)$ is the principal symbol of the Laplace operator in a local chart $V$, for all $x_{0} \in V$, there exists $\xi_{0}$ and $\tau_{0}>0$ such that $a\left(x_{0}, \xi_{0}+i \tau_{0} d_{x} \varphi\left(x_{0}\right)\right)=0$. Then, the symbol $b=a^{2}$ vanishes at second order at $\left(x_{0}, \xi_{0}+i \tau_{0} d_{x} \varphi\left(x_{0}\right)\right.$. Hence, we cannot hope for a Carleman estimate for $B$ with a loss of less than one full derivative. In fact, such an estimate can be obtained by using twice in cascade the Carleman estimate for the Laplace operator. This is consistent, as the estimate for the Laplace operator exhibits a loss a half derivative in dimension greater than 1 (if $\varphi$ is chosen such that sub-ellipticity holds - see [LL12]).

In dimension one, however, $B=D_{x}^{4}$ and the conjugated operator $\left(D_{x}+i \tau d \varphi(x)\right)^{4}$ is elliptic (in the sense of semi-classical operators) if $d \varphi(x) \neq 0$ in $\Omega$. Then, the resulting Carleman estimate is characterized by no derivative loss.

Concerning the operator $P=D_{s}^{4}+B$ in $Z=\left(0, S_{0}\right) \times \Omega$, that is central in the present article, we write $P=P_{1} P_{2}$ with $P_{k}=(-1)^{k} i D_{s}^{2}+A$. Setting $P_{k, \varphi}=e^{\tau \varphi} P_{k} e^{-\tau \varphi}$, with semi-classical principal symbols given by

$$
p_{k, \varphi}(z, \zeta, \tau)=(-1)^{k} i\left(\sigma+i \tau \partial_{s} \varphi(z)\right)^{2}+a\left(x, \xi+i \tau d_{x} \varphi(z)\right), \quad k=1,2,
$$

where $z=(s, x) \in Z$ and $\zeta=(\sigma, \xi) \in \mathbb{R}^{1+d}=\mathbb{R}^{N}$. Let $d \geq 2$. If, for some $z_{0} \in Z$, we have $\partial_{s} \varphi\left(z_{0}\right)=0$, if we choose $\xi_{0} \in \mathbb{R}^{d}$ and $\tau_{0}>0$ such that $a\left(x_{0}, \xi_{0}+i \tau_{0} d_{x} \varphi\left(z_{0}\right)\right)=0$, then for $\sigma_{0}=0$, we have $\zeta_{0}=\left(0, \xi_{0}\right) \in \mathbb{R}^{N}$ and $\theta_{0}=\left(0, \xi_{0}\right)+i \tau_{0}\left(0, d_{x} \varphi\left(z_{0}\right)\right)$ and $p_{k, \varphi}\left(z_{0}, \zeta_{0}, \tau_{0}\right)=p_{k}\left(z_{0}, \theta_{0}\right)=0$ and $d_{z, \zeta} p\left(z_{0}, \theta_{0}\right)=0$, where $p$ and $p_{k}$ are the principal symbols of $P$ and $P_{k}, k=1,2$. Hence, in a neighborhood of $z_{0}$, Proposition 1.6 applies.

This situation occurs in Cases (i) and (iii) described in Section 1.3 and Figure 1. In the neighborhoods $V_{1}$ and $V_{3}$ introduced there, we have points where $\partial_{s} \varphi$ vanishes (as can observed by the shapes of the level sets of $\varphi$ in Figure 1). This explains why we can only obtain estimates with a loss of one full derivative for those cases. In case (ii), however, this does not occur, and there we obtain an estimation with only a loss of a half derivative.

1.5. Some perspectives. The present article deals with the natural "clamped" boundary conditions, that is, homogeneous Dirichlet and Neumann conditions simultaneously. In the light of the results obtained here and those that can be obtained for very general boundary conditions of Lopatinskii type in [Tat96, BL15], for instance for unique continuation through the derivation of Carleman estimates at the boundary for general elliptic operators with complex coefficient in cases where the sub-ellipticity property hold, one is inclined to attempt to prove estimates similar to those proven in the present article, in the case of an operator, such as the operator $P=D_{s}^{4}+B$ studied here, for which the sub-ellipticity condition cannot hold everywhere and for general boundary conditions of Lopatinskii type.

Here, we considerer the bi-Laplace operator $B=\Delta^{2}$. It would be of interest to consider more general polyharmonic operators such as $\Delta^{k}, k \in \mathbb{N}$, on $\Omega$ along with natural boundary conditions, e.g.,

$$
u_{\mid \partial \Omega}=0, \ldots, \partial_{v}^{k-1} u_{\mid \partial \Omega}=0,
$$

or more general Lopatinskii type conditions. 
1.6. Notation. We shall use some spaces of smooth functions in the closed half space. We set

$$
\mathscr{S}\left(\overline{\mathbb{R}_{+}^{N}}\right)=\left\{u_{\mid \mathbb{R}_{+}^{N}} ; u \in \mathscr{S}\left(\mathbb{R}^{N}\right)\right\} .
$$

The reader needs to be warned that in some sections $z \in \mathbb{R}^{N}$ will denote $(x, s)$, with $x \in \mathbb{R}^{d}=$ $\mathbb{R}^{N-1}$ and $s \in \mathbb{R}$, and thus, there, $z_{N}=s$. This is the case in Section 3. In other sections, $z$ will denote $(s, x)$, and thus there $z_{N}=x_{d}$. This is the case of Section 4 and Appendices A.2 and B.

Some specific notation for semi-classical tangential operators will be introduced in Section 3.1, and they allow us to derive the Carleman estimate for $D_{s}^{4}+B$ at the boundary $\{0\} \times \Omega$ (Cases (i) above). Semi-classical calculus is characterized by the presence of a large parameter denoted by $\tau$ here, that is precisely the large parameter that appears in the Carleman estimates (for readers familiar with semi-classical analysis this is done by taking $\tau=1 / h$ where $h$ is the Planck constant.)

A special class of semi-classical calculus is also introduced in Section 4.1 and is characterized by three parameters. This calculus is essential in the proof of the Carleman estimate for $D_{s}^{4}+B$ at the boundary $\left(0, S_{0}\right) \times \partial \Omega$ (Case (iii) above).

In this article, when the constant $C$ is used, it refers to a constant that is independent of the semi-classical parameters, e.g. $\tau, \gamma, \varepsilon$. Its value may however change from one line to another. If we want to keep track of the value of a constant we shall use another letter.

For concision, we use the notation $\lesssim$ for $\leq C$, with a constant $C>0$. We also write $a \asymp b$ to denote $a \lesssim b \lesssim a$. As done above, we shall use the notation $\|$.$\| for functions in the interior of the$ domain and $|$.$| for functions on the boundaries.$

We finish this introductory section by stating some basic properties of the "clamped" bi-Laplace operator that will be used at places in this article (some were implicitly used above).

1.7. Some basic properties of the bi-Laplace operator. We recall here some facts on the "clamped" bi-Laplace operator. We define the operator $B=\Delta^{2}$ on $L^{2}(\Omega)$ with domain $D(B)=H^{4}(\Omega) \cap H_{0}^{2}(\Omega)$.

Proposition 1.8. The operator $(B, D(B))$ is selfadjoint on $L^{2}(\Omega)$ and maximal monotone.

In particular, if $\mu \geq 0$, there exists $C>0$ such that, for any $f \in L^{2}(\Omega)$, there exists a unique $u \in D(B)$ such that

$$
\Delta^{2} u+\mu u=f \text {, and }\|u\|_{H^{4}(\Omega)} \leq C\|f\|_{L^{2}(\Omega)} .
$$

This can be proven by first finding a unique solution in $H_{0}^{2}(\Omega)$ with the Lax-Milgram theorem and then applying Theorem 20.1.2 in [Hör85b, Section 20.1]. Note in particular that $\left\|\Delta^{2} u\right\|_{L^{2}}$ is a equivalent norm on $H^{4}(\Omega) \cap H_{0}^{2}(\Omega)$ by (1.15).

As a consequence of Proposition 1.8 we have the existence of a Hilbert basis for $L^{2}(\Omega)$ made of eigenfunctions.

Corollary 1.9. There exist $\left(\mu_{j}\right)_{j \in \mathbb{N}} \subset \mathbb{R}$, and $\left(\varphi_{j}\right)_{j \in \mathbb{N}} \subset D(B)$ such that

$$
0<\mu_{1} \leq \mu_{2} \leq \cdots \leq \mu_{j} \leq \cdots, \quad \lim _{j \rightarrow \infty} \mu_{j}=+\infty, \quad B \varphi_{j}=\mu_{j} \varphi_{j},
$$

and the family $\left(\varphi_{j}\right)_{j}$ forms a Hilbert basis for $L^{2}(\Omega)$.

Corollary 1.10. The operator $(B, D(B))$ generates an analytic $C_{0}$-semigroup $S(t)$ on $L^{2}(\Omega)$.

For $T>0, y_{0} \in L^{2}(\Omega)$, and $f \in L^{2}\left(0, T ; H^{-2}(\Omega)\right)$, there exists a unique

$$
y \in L^{2}\left([0, T] ; H_{0}^{2}(\Omega)\right) \cap \mathscr{C}\left([0, T] ; L^{2}(\Omega)\right) \cap H^{1}\left(0, T ; H^{-2}(\Omega)\right),
$$

given by $y(t)=S(t) y_{0}+\int_{0}^{t} S(t-s) f(s) d s$, such that

$$
\partial_{t} y+\Delta^{2} y=f \text { for } t \in(0, T) \text { a.e., } \quad y_{\mid t=0}=y_{0}
$$


For semigroup theory we refer the reader to [Paz83].

For the operator $\mathcal{B}$ defined in (1.4) we have the following property.

Proposition 1.11. The spectrum of $\mathcal{B}$ is contained in $\{z \in \mathbb{C} ; \operatorname{Re}(z)>0\}$. Moreover, for $z \in \mathbb{C}$ such that $\operatorname{Re} z<0$, we have

$$
\left\|\left(z \operatorname{Id}_{\mathscr{H}}-\mathcal{B}\right) U\right\|_{\mathscr{H}} \geq|\operatorname{Re} z|\|U\|_{\mathscr{H}}, \quad U \in D(\mathcal{B}),
$$

with $\mathscr{H}=H_{0}^{2}(\Omega) \times L^{2}(\Omega)$.

With the Hille-Yoshida theorem [Paz83, Theorem 3.1, Chapter 1] we then have the following results.

Corollary 1.12. The unbounded operator $(\mathcal{B}, D(\mathcal{B}))$ generates a $C_{0}$-semigroup of contraction $\Sigma(t)$ on $\mathscr{H}$.

Corollary 1.13. For $\left(y_{0}, y_{1}\right) \in D(\mathcal{B})$ there exists a unique

$$
y \in \mathscr{C}^{2}\left([0,+\infty) ; L^{2}(\Omega)\right) \cap \mathscr{C}^{1}\left([0,+\infty) ; H_{0}^{2}(\Omega)\right) \cap \mathscr{C}^{0}([0,+\infty) ; D(B)),
$$

such that

$$
\partial_{t}^{2} y+\Delta^{2} y+\alpha \partial_{t} y=0 \text { in } L^{\infty}\left((0,+\infty) ; L^{2}(\Omega)\right), \quad y_{\mid t=0}=y_{0}, \partial_{t} y_{\mid t=0}=y_{1} .
$$

The solution is given by the first component of $\Sigma(t) Y_{0}$ with $Y_{0}=\left(y_{0}, y_{1}\right)$. The energy $t \mapsto E(y)(t)$ with

$$
E(y)(t)=\frac{1}{2}\left\|\partial_{t} y(t)\right\|_{L^{2}(\Omega)}^{2}+\frac{1}{2}\|\Delta y(t)\|_{L^{2}(\Omega)}^{2},
$$

is nonincreasing: for $0 \leq t_{1} \leq t_{2}$ we have $E(y)\left(t_{2}\right)-E(y)\left(t_{1}\right)=-\int_{t_{1}}^{t_{2}}\left\|\alpha^{1 / 2} \partial_{t} y(t)\right\|_{L^{2}(\Omega)}^{2} d t$.

\section{ESTIMATE AWAY FROM BOUNDARIES}

For operators exhibiting at most double (complex) roots, estimates can be found in the proof of Theorem 28.1.8 in [Hör85a]. Here, the structure of the operator $P$ is explicit which allows one to expose the argumentation in a self contained yet short presentation.

2.1. Simple-characteristic property of second-order factors. We consider the augmented operator $P=D_{s}^{4}+B$ in $Z=\left(0, S_{0}\right) \times \Omega$, remaining away from boundaries here. We write

$$
P=P_{1} P_{2}, \quad \text { with } P_{k}=(-1)^{k} i D_{s}^{2}+A .
$$

Here, we show that $P_{1}$ and $P_{2}$ both satisfy the so-called simple characteristic property in the case of a weight function whose differential does not vanish.

Let $\ell(z, \zeta)$, with $(z, \zeta) \in \mathbb{R}^{N} \times \mathbb{R}^{N}$, be polynomial of degree $m$ in $\zeta$, with smooth coefficient in $z$. For $z \mapsto M(z) \in \mathbb{R}^{N} \backslash\{0\}$, we introduce the map

$$
\begin{aligned}
\rho_{z, \zeta, M}: \mathbb{R}_{+} & \rightarrow \mathbb{C}, \\
\theta & \mapsto \ell(z, \zeta+i \theta M(z)) .
\end{aligned}
$$

Definition 2.1. Let $W$ be an open set of $\mathbb{R}^{N}$. We say that $\ell$ satisfies the simple-characteristic property in direction $M$ in $\bar{W}$ if, for all $z \in \bar{W}$, we have $\zeta=0$ and $\theta=0$ when the map $\rho_{z, \zeta, M}$ has a double root.

We can formulate this condition as follows

$$
\ell(z, \zeta+i \theta M(z))=d_{\zeta} \ell(z, \zeta+i \theta M(z))(M(z))=0 \quad \Rightarrow \quad \zeta=0, \theta=0 .
$$


Lemma 2.2. Let $W$ be an open set of $\mathbb{R}^{N}$. If $N \geq 3$ and $\ell(z, \zeta)$ is of order two (with complex coefficients) and elliptic for $z \in \bar{W}$, then for any map $z \mapsto M(z) \in \mathbb{R}^{N} \backslash\{0\}, \ell$ satisfies the simplecharacteristic property in direction $M$ in $\bar{W}$.

Proof. The proof can be adapted from classical ideas (see [LM68, proof of Proposition 1.1, Chapter 2] or [Hör83]). We consider the polynomial $f_{z, \zeta, M}(t)=\ell(z, \zeta+t M(z))$ where $t$ is a complex variable, for $z \in \bar{W}, \zeta \in \mathbb{R}^{N}$.

If $\zeta$ is colinear to $M(z)$, e.g. $\zeta=\alpha M(z)$ then $f_{z, \zeta, M}(t)=(\alpha+t)^{2} \ell(z, M(z))$. Because of the ellipticity of $\ell, \ell(z, M(z)) \neq 0$, and we only have $t=-\alpha$ as a double real root for $f$.

We set $J=\mathbb{R}^{N} \backslash \operatorname{Span}(M(z))$. Note that $z$ is fixed here and $\operatorname{Span}(M(z))$ is a vector line. The set $J$ is connected as $N \geq 3$. Let now $\zeta \in J$, that is, $\zeta$ is not colinear to $M(z)$. As $\ell$ is elliptic, the roots of $f_{z, \zeta, M}$ cannot be real numbers. We denote by $\mathrm{m}^{+}(\zeta)$ and $\mathrm{m}^{-}(\zeta)$ the number of roots with positive and negative imaginary parts, respectively. We have $2=m^{+}(\zeta)+m^{-}(\zeta)$. Since roots are continuous w.r.t. $\zeta$ and cannot be real, they remain in the upper- or lower-half complex plane as $\zeta$ varies in $J$, as $J$ is connected, meaning that $m^{+}$and $m^{-}$are then invariant. In particular, $m^{+}(\zeta)=m^{+}(-\zeta)$ and $m^{-}(\zeta)=m^{-}(-\zeta)$. Observing however, that if $t_{0}$ is a root of $t \mapsto \ell(z, \zeta+t M(z))$ then $-t_{0}$ is a root of $t \mapsto \ell(z,-\zeta+t M(z))$, we find that $m^{+}(\zeta)=m^{-}(-\zeta)$. This gives $m^{+}(\zeta)=m^{-}(\zeta)=1$. Hence, complex roots are simple.

In any case, we see that if the map $\theta \mapsto \rho_{z, \zeta, M}=f_{z, \zeta, M}(i \theta)$ has a double real root $\theta_{0}$ then $\theta_{0}=0$ and $\zeta=0$. The simple-characteristic property is thus fulfilled.

If we consider a weight function $\psi=\psi(s, x)$, for the operators $P_{k}, k=1,2$, introduced in (2.1), we have the following proposition.

Proposition 2.3. Let $k=1$ or 2 . Assume that $d \psi \neq 0$ in $\overline{\left(0, S_{0}\right) \times \Omega}$. Then, $P_{k}$ satisfies the simple-characteristic property in direction $d \psi$ in $\overline{\left(0, S_{0}\right) \times \Omega}$.

Proof. Here, the dimension is $N=d+1$. The case $d \geq 2$ is treated in Lemma 2.2. It only remains to treat the case of dimension $d=1$. Then, the principal symbol of $A$ reads $a(x, \xi)=\alpha(x) \xi^{2}$, with $\alpha(x) \geq C>0$. We set $M(z)=\left(M_{\sigma}(z), M_{\xi}(z)\right)=d \psi(z) \in \mathbb{R}^{N} \backslash\{0\}$. We write $\rho$ in place of $\rho_{\left(z_{0}, \zeta_{0}, M\right)}$ for concision.

With $\zeta=(\sigma, \xi)$, we have

$$
\begin{aligned}
\rho(\theta) & =p_{k}\left(z_{0}, \zeta+i \theta M\right)=(-1)^{k} i\left(\sigma+i \theta M_{\sigma}\right)^{2}+\alpha\left(x_{0}\right)\left(\xi+i \theta M_{\xi}\right)^{2} \\
& =\alpha\left(x_{0}\right) \xi^{2}-\alpha\left(x_{0}\right)\left(\theta M_{\xi}\right)^{2}-2(-1)^{k} \theta \sigma M_{\sigma}+i\left((-1)^{k} \sigma^{2}-(-1)^{k}\left(\theta M_{\sigma}\right)^{2}+2 \theta \alpha\left(x_{0}\right) \xi M_{\xi}\right) .
\end{aligned}
$$

We thus have $\frac{1}{2} \partial_{\theta} \rho(\theta)=-\alpha\left(x_{0}\right) \theta M_{\xi}^{2}-(-1)^{k} \sigma M_{\sigma}+i\left(\alpha\left(x_{0}\right) \xi M_{\xi}-(-1)^{k} \theta M_{\sigma}^{2}\right)$. Assuming that $M_{\sigma} \neq 0$, if $\partial_{\theta} \rho=0$ we find

$$
\theta=(-1)^{k} \frac{\alpha\left(x_{0}\right) \xi M_{\xi}}{M_{\sigma}^{2}}, \quad \text { and } \sigma=-\alpha\left(x_{0}\right)^{2} \xi \frac{M_{\xi}^{3}}{M_{\sigma}^{3}} .
$$

This yields $\rho=\alpha\left(x_{0}\right) \xi^{2}\left(1+(-1)^{k} i \alpha\left(x_{0}\right) M_{\xi}^{2} / M_{\sigma}^{2}\right)\left(1+\alpha\left(x_{0}\right)^{2} M_{\xi}^{4} / M_{\sigma}^{4}\right)$. In this case, we thus have $\rho=\partial_{\theta} \rho=0$ if and only if $\theta=0$ and $\zeta=(\sigma, \xi)=(0,0)$.

We assume now that $M_{\sigma}=0$. Since $M \neq 0$, we find that $\partial_{\theta} \rho=0$ implies $\theta=0$ and $\xi=0$. Then $\rho=0$ gives $\sigma=0$. Hence, in any case, the simple characteristic property is fulfilled.

2.2. Local Carleman estimates away from boundaries. Let $V$ be an open subset of $Z=\left(0, S_{0}\right) \times$ $\Omega$. We set $z=(s, x)$. Let $L=L\left(z, D_{z}\right)$ be a differential operator of order $m$, with smooth principal symbol, $\ell(z, \zeta)$. 
Definition 2.4. Let $\varphi(z)$ be defined and smooth in $\bar{V}$ and such that $|d \varphi| \geq C>0$. We say that the couple $(L, \varphi)$ satisfies the sub-ellipticity condition in $\bar{V}$ if we have

$$
\begin{aligned}
& \ell(z, \zeta+i \tau \varphi(z))=0 \Rightarrow \\
& \quad \frac{1}{2 i}\{\overline{\ell(z, \zeta+i \tau d \varphi(z))}, \ell(z, \zeta+i \tau d \varphi(z))\}=\{\operatorname{Re} \ell(z, \zeta+i \tau d \varphi(z)), \operatorname{Im} \ell(z, \zeta+i \tau d \varphi(z))\}>0,
\end{aligned}
$$

for all $z \in \bar{V}$ and $\zeta \in \mathbb{R}^{N}$ and $\tau>0$.

Let $\psi(z)$ be smooth in $\bar{V}$ and such that $|d \psi| \geq C>0$ in $\bar{V}$. We define $\varphi(z)=\exp (\gamma \psi(z))$. Sub-ellipticity for the couple $\left(P_{k}, \varphi\right)$ can be easily achieved by the following lemma.

Lemma 2.5. The couple $\left(P_{k}, \varphi\right)$ satisfies the sub-ellipticity condition in $\bar{V}$ for $\gamma>0$ chosen sufficiently large.

Proof. By Proposition 2.3 we see that $P_{k}$ satisfies the simple-characteristic property in direction $d \psi$ in $\bar{V}$. This implies that $\psi$ is strongly pseudo-convex with respect to $P_{k}$ in the sense given in [Hör85a, Section 28.3] at every point in $V$. We then obtain that the couple $\left(P_{k}, \varphi\right)$ satisfies the subellipticity condition in $\bar{V}$ for $\gamma>0$ chosen sufficiently large by Proposition 28.3.3 in [Hör85a].

A consequence of the sub-ellipticity property is the following Carleman estimate for $P_{k}$ in $V$, that is, away from boundaries.

Proposition 2.6. Let $k=1$ or 2 . Let $\varphi=\exp (\gamma \psi)$ with $|d \psi| \geq C>0$ in $\bar{V}$. For $\gamma>0$ chosen sufficiently large, there exist $C>0$ and $\tau_{0}$ such that

$$
\sum_{|\alpha| \leq 2} \tau^{3 / 2-|\alpha|}\left\|e^{\tau \varphi} D_{z}^{\alpha} u\right\|_{L^{2}(Z)} \leq C\left\|e^{\tau \varphi} P_{k} u\right\|_{L^{2}(Z)},
$$

for $\tau \geq \tau_{0}$ and $u \in \mathscr{C}_{c}^{\infty}(V)$.

We refer to [Hör85a, Theorem 28.2.3] for a proof. In fact, to incorporate the term associated with $|\alpha|=2$ see [Hör63]. This estimate is characterized by the loss of a half derivative.

From this estimate for $P_{k}, k=1,2$, we deduce the following estimate for the operator $P=P_{1} P_{2}$.

Proposition 2.7. Let $\varphi=\exp (\gamma \psi)$ with $|d \psi| \geq C>0$ in $\bar{V}$. For $\gamma>0$ chosen sufficiently large, there exist $C>0$ and $\tau_{0}$ such that

$$
\sum_{|\alpha| \leq 4} \tau^{3-|\alpha|}\left\|e^{\tau \varphi} D_{z}^{\alpha} u\right\|_{L^{2}(Z)} \leq C\left\|e^{\tau \varphi} P u\right\|_{L^{2}(Z)}
$$

for $\tau \geq \tau_{0}$ and $u \in \mathscr{C}_{c}^{\infty}(V)$.

This estimate is characterized by the loss of a full derivative.

Proof. With the estimate of Proposition 2.6 for the operator $P_{1}$ applied to $P_{2} u \in \mathscr{C}^{\infty}(V)$ we have

$$
\sum_{|\alpha| \leq 2} \tau^{3 / 2-|\alpha|}\left\|e^{\tau \varphi} D_{z}^{\alpha} P_{2} u\right\|_{L^{2}(Z)} \lesssim\left\|e^{\tau \varphi} P u\right\|_{L^{2}(Z)} .
$$

Observing that $\left[D_{z}^{\alpha}, P_{2}\right]$ is a differential operator of order $1+|\alpha|$ we obtain

$$
\sum_{|\alpha| \leq 2} \tau^{3 / 2-|\alpha|}\left\|e^{\tau \varphi} P_{2} D_{z}^{\alpha} u\right\|_{L^{2}(Z)} \lesssim\left\|e^{\tau \varphi} P u\right\|_{L^{2}(Z)}+\sum_{|\beta| \leq 3} \tau^{5 / 2-|\beta|}\left\|e^{\tau \varphi} D_{z}^{\beta} u\right\|_{L^{2}(Z)} .
$$

Applying now the estimate of Proposition 2.6 for the operator $P_{2}$ to $D_{z}^{\alpha} u \in \mathscr{C}^{\infty}(V)$ we obtain

$$
\sum_{|\delta| \leq 2} \tau^{3 / 2-|\delta|}\left\|e^{\tau \varphi} D_{z}^{\delta+\alpha} u\right\|_{L^{2}(Z)} \lesssim\left\|e^{\tau \varphi} P_{2} D_{z}^{\alpha} u\right\|_{L^{2}(Z)} .
$$


With (2.4) we then obtain

$$
\begin{aligned}
\sum_{|\alpha| \leq 4} \tau^{3-|\alpha|}\left\|e^{\tau \varphi} D_{z}^{\alpha} u\right\|_{L^{2}(Z)} & \asymp \sum_{|\delta| \leq 2|\alpha| \leq 2} \tau^{3-|\delta|-|\alpha|}\left\|e^{\tau \varphi} D_{z}^{\delta+\alpha} u\right\|_{L^{2}(Z)} \\
& \lesssim\left\|e^{\tau \varphi} P u\right\|_{L^{2}(Z)}+\sum_{|\beta| \leq 3} \tau^{5 / 2-|\beta|}\left\|e^{\tau \varphi} D_{z}^{\beta} u\right\|_{L^{2}(Z)} .
\end{aligned}
$$

We then conclude by choosing $\tau>0$ sufficiently large.

\section{Estimate at THE Boundary $\{s=0\}$}

3.1. Tangential semi-classical calculus and associated Sobolev norms. Considering boundary problems, we shall locally use coordinates so that the geometry is that of the half space

$$
\overline{\mathbb{R}_{+}^{N}}=\left\{z \in \mathbb{R}^{N}, z_{N}>0\right\}, \quad z=\left(z^{\prime}, z_{N}\right) \text { with } z^{\prime} \in \mathbb{R}^{N-1}, z_{N} \in \mathbb{R} .
$$

We shall use the notation $\varrho=(z, \zeta, \tau)$ and $\varrho^{\prime}=\left(z, \zeta^{\prime}, \tau\right)$ in this section. (This notation is not to be confused with that introduced and used in Section 4 and Appendix B.)

Let $a\left(\varrho^{\prime}\right) \in \mathscr{C}^{\infty}\left(\overline{\mathbb{R}_{+}^{N}} \times \mathbb{R}^{N-1}\right)$, with $\tau$ as a parameter in $[1,+\infty)$ and $m \in \mathbb{R}$, be such that, for all multi-indices $\alpha, \beta$, we have

$$
\left|\partial_{z}^{\alpha} \partial_{\zeta^{\prime}}^{\beta} a\left(\varrho^{\prime}\right)\right| \leq C_{\alpha, \beta} \lambda_{\mathrm{T}, \tau}^{m-|\beta|}, \quad z \in \overline{\mathbb{R}_{+}^{N}}, \zeta^{\prime} \in \mathbb{R}^{N-1}, \tau \in[1,+\infty),
$$

where $\lambda_{\mathrm{T}, \tau}^{2}=\left|\zeta^{\prime}\right|^{2}+\tau^{2}$. We write $a \in S_{\mathrm{T}, \tau}^{m}$. We also define $S_{\mathrm{T}, \tau}^{-\infty}=\cap_{r \in \mathbb{R}} S_{\mathrm{T}, \tau}^{r}$. For $a \in S_{\mathrm{T}, \tau}^{m}$ we call principal symbol, $\sigma(a)$, the equivalence class of $a$ in $S_{\mathrm{T}, \tau}^{m} / S_{\mathrm{T}, \tau}^{m-1}$. Note that we have $\lambda_{\mathrm{T}, \tau}^{m} \in S_{\mathrm{T}, \tau}^{m}$.

If $a\left(\varrho^{\prime}\right) \in S_{\mathrm{T}, \tau}^{m}$, we set

$$
\mathrm{Op}_{\mathrm{T}}(a) u(z):=(2 \pi)^{-(N-1)} \int_{\mathbb{R}^{N-1}} e^{i\left(z^{\prime}, \zeta^{\prime}\right)} a\left(\varrho^{\prime}\right) \hat{u}\left(\zeta^{\prime}, z_{N}\right) d \zeta^{\prime},
$$

for $u \in \mathscr{S}\left(\overline{\mathbb{R}_{+}^{N}}\right)$, where $\hat{u}$ is the partial Fourier transform of $u$ with respect to the tangential variables $z^{\prime}$. We denote by $\Psi_{\mathrm{T}, \tau}^{m}$ the set of these pseudo-differential operators. For $A \in \Psi_{\mathrm{T}, \tau}^{m}, \sigma(A)=\sigma(a)$ will be its principal symbol in $S_{\mathrm{T}, \tau}^{m} / S_{\mathrm{T}, \tau}^{m-1}$. We also set $\Lambda_{\mathrm{T}, \tau}^{m}=\mathrm{Op}_{\mathrm{T}}\left(\lambda_{\mathrm{T}, \tau}^{m}\right), m \in \mathbb{R}$.

Let $m \in \mathbb{N}$ and $m^{\prime} \in \mathbb{R}$. If we consider $a$ of the form

$$
a(\varrho)=\sum_{j=0}^{m} a_{j}\left(\varrho^{\prime}\right) \zeta_{N}^{j}, \quad a_{j} \in S_{\mathrm{T}, \tau}^{m+m^{\prime}-j},
$$

we define $\mathrm{Op}(a):=\sum_{j=0}^{m} \mathrm{Op}_{\mathrm{T}}\left(a_{j}\right) D_{z_{N}}^{j}$. We write $a \in S_{\tau}^{m, m^{\prime}}$ and $\mathrm{Op}(a) \in \Psi_{\tau}^{m, m^{\prime}}$.

We define the following norm, for $m \in \mathbb{N}$ and $m^{\prime} \in \mathbb{R}$,

$$
\begin{aligned}
& \|u\|_{m, m^{\prime}, \tau} \asymp \sum_{j=0}^{m}\left\|\Lambda_{\mathrm{T}, \tau}^{m+m^{\prime}-j} D_{z_{N}}^{j} u\right\|_{+} \\
& \|u\|_{m, \tau}=\|u\|_{m, 0, \tau} \asymp \sum_{j=0}^{m}\left\|\Lambda_{\mathrm{T}, \tau}^{m-j} D_{z_{N}}^{j} u\right\|_{+}, \quad u \in \mathscr{S}\left(\overline{\mathbb{R}_{+}^{N}}\right),
\end{aligned}
$$

where $\|.\|_{+}:=\|\cdot\|_{L^{2}\left(\mathbb{R}_{+}^{N}\right)}$. We have

$$
\|u\|_{m, \tau} \asymp \sum_{\substack{|\alpha| \leq m \\ \alpha \in \mathbb{N}^{N}}} \tau^{m-|\alpha|}\left\|D^{\alpha} u\right\|_{+}
$$

and in the case $m^{\prime} \in \mathbb{N}$ we have

$$
\|u\|_{m, m^{\prime}, \tau} \asymp \sum_{\alpha_{N} \leq m} \sum_{\substack{|\alpha| \leq m+m^{\prime} \\ \alpha=\left(\alpha^{\prime}, \alpha_{N}\right) \in \mathbb{N}^{N}}} \tau^{m+m^{\prime}-|\alpha|}\left\|D^{\alpha} u\right\|_{+} .
$$


If $m, m^{\prime} \in \mathbb{N}$ and $m^{\prime \prime}, m^{\prime \prime \prime} \in \mathbb{R}$, and if $a \in S_{\mathrm{T}, \tau}^{m^{\prime \prime}}$, then we have

$$
\left\|\mathrm{Op}_{\mathrm{T}}(a) u\right\|_{m^{\prime}, m^{\prime \prime \prime}, \tau} \leq C\|u\|_{m^{\prime}, m^{\prime \prime}+m^{\prime \prime \prime}, \tau}, \quad u \in \mathscr{S}\left(\overline{\mathbb{R}_{+}^{N}}\right) .
$$

If $a \in S_{\mathrm{T}, \tau}^{m, m^{\prime \prime}}$, then we have

$$
\left\|\mathrm{Op}_{\mathrm{T}}(a) u\right\|_{m^{\prime}, m^{\prime \prime \prime}, \tau} \leq C\|u\|_{m+m^{\prime}, m^{\prime \prime}+m^{\prime \prime \prime}, \tau}, \quad u \in \mathscr{S}\left(\overline{\mathbb{R}_{+}^{N}}\right) .
$$

The following argument will be used on many occasions in what follows, for $m \in \mathbb{N}, m^{\prime}, \ell \in \mathbb{R}$, with $\ell>0$,

$$
\|w\|_{m, m^{\prime}, \tau} \ll\|w\|_{m, m^{\prime}+\ell, \tau} .
$$

At the boundary $\left\{z_{N}=0\right\}$ we define the following norms, for $m \in \mathbb{N}$ and $m^{\prime} \in \mathbb{R}$,

$$
|\operatorname{tr}(u)|_{m, m^{\prime}, \tau}^{2}=\sum_{j=0}^{m}\left|\Lambda_{\mathrm{T}, \tau}^{m-j+m^{\prime}} D_{z_{N}}^{j} u_{z_{N}=0^{+}}\right|_{L^{2}\left(\mathbb{R}^{N-1}\right)}^{2}, \quad u \in \mathscr{S}\left(\overline{\mathbb{R}_{+}^{N}}\right) .
$$

3.2. Statement of the Carleman estimate. In this section, we consider $z=(x, s) \in \mathbb{R}^{N}$ with $x \in \mathbb{R}^{d}$ and $s \in \mathbb{R}$. We also set $Z=\Omega \times\left(0, S_{0}\right)$. We write $x=z^{\prime}$ and $s=z_{N}$, in connexion with the notation introduced for the tangential calculus in Section 3.1.

Let $z_{0}=\left(x_{0}, 0\right)$ with $x_{0} \in \Omega$. We consider a function $\psi \in \mathscr{C}^{\infty}\left(\mathbb{R}^{N}\right)$ such that $\partial_{s} \psi(z) \leq-C<0$ in a bounded open neighborhood $V$ of $z_{0}$ in $\mathbb{R} \times \Omega$. We then set $\varphi(z)=e^{\gamma \psi(z)}$.

Using the notation introduced in Section 3.1 for semi-classical norms, we have the following Carleman estimate at the boundary $\Omega \times\{0\}$ for functions defined in $\{s \geq 0\} \cap V$.

Theorem 3.1. Let $P=D_{s}^{4}+B=D_{s}^{4}+\Delta^{2}$ on $Z=\Omega \times\left(0, S_{0}\right)$. Let $W$ be an open set of $\mathbb{R}^{N}$ such that $W \Subset V$. For $\gamma>0$ chosen sufficiently large, there exist $\tau_{0} \geq 1$ and $C>0$ such that

$$
\sum_{|\alpha| \leq 4} \tau^{7 / 2-|\alpha|}\left\|e^{\tau \varphi} D_{s, x}^{\alpha} u\right\|_{L^{2}(Z)} \leq C\left(\left\|e^{\tau \varphi} P u\right\|_{L^{2}(Z)}+\sum_{j=0}^{3}\left|\operatorname{tr}\left(e^{\tau \varphi} D_{s}^{j} u\right)\right|_{0,7 / 2-j, \tau}\right),
$$

for $\tau \geq \tau_{0}$ and for $u=w_{\mid Z}$, with $w \in \mathscr{C}_{c}^{\infty}\left(\mathbb{R}^{d} \times \mathbb{R}\right)$ and $\operatorname{supp}(w) \subset W$.

This Carleman estimate is characterized by the loss of a half derivative.

Corollary 3.2. Let $P=D_{s}^{4}+B=D_{s}^{4}+\Delta^{2}$ on $Z=\Omega \times\left(0, S_{0}\right)$. Let $W$ be an open set of $\mathbb{R}^{N}$ such that $W \Subset V$. For $\gamma>0$ chosen sufficiently large, there exist $\tau_{0} \geq 1$ and $C>0$ such that

$$
\sum_{|\alpha| \leq 3} \tau^{7 / 2-|\alpha|}\left\|e^{\tau \varphi} D_{s, x}^{\alpha} u\right\|_{L^{2}(Z)} \leq C\left(\left\|e^{\tau \varphi} P u\right\|_{L^{2}(Z)}+\tau^{1 / 2} \sum_{j=0}^{3}\left|\operatorname{tr}\left(e^{\tau \varphi} D_{s}^{j} u\right)\right|_{0,3-j, \tau}\right),
$$

for $\tau \geq \tau_{0}$ and for $u=w_{\mid Z}$, with $w \in \mathscr{C}_{c}^{\infty}\left(\mathbb{R}^{d} \times \mathbb{R}\right)$ and $\operatorname{supp}(w) \subset W$.

Proofs are given below.

3.3. Sub-ellipticity property. As in Section 2.1 , we write $P=P_{1} P_{2}$ with $P_{k}=(-1)^{k} i D_{s}^{2}+A$, and $P_{\varphi}=e^{\tau \varphi} P e^{-\tau \varphi}=Q_{1} Q_{2}$ with $Q_{k}=e^{\tau \varphi} P_{k} e^{-\tau \varphi}$. The principal symbol of $q_{k}$, in the sense of semi-classical operators, is given by

$$
q_{k}(z, \zeta, \tau)=(-1)^{k} i\left(\sigma+i \hat{\tau}_{\sigma}\right)^{2}+a\left(x, \xi+i \hat{\tau}_{\xi}\right), \quad \hat{\tau}(z, \tau)=\left(\hat{\tau}_{\xi}, \hat{\tau}_{\sigma}\right)=\tau d \varphi \in \mathbb{R}^{N},
$$

where $a(x, \xi)$ denotes the principal symbol of the Laplace operator $A$.

Recalling the definition of the semi-classical characteristic set of a (pseudo-)differential operator $A$, with principal symbol $a(\varrho)$,

$$
\operatorname{char}(A)=\left\{\varrho=(z, \zeta, \tau) \in \bar{V} \times \mathbb{R}^{N} \times \mathbb{R}_{+} ;(\zeta, \tau) \neq(0,0) \text {, and } a(\varrho)=0\right\},
$$

we have the following results for the characteristic sets of $Q_{k}, k=1,2$. 
Lemma 3.3. In $\bar{V}$, we have $\operatorname{char}\left(Q_{1}\right) \cap \operatorname{char}\left(Q_{2}\right)=\emptyset$.

Proof. Let $\varrho=(z, \zeta, \tau) \in \bar{V} \times \mathbb{R}^{N} \times \mathbb{R}_{+}$, with $(\zeta, \tau) \neq(0,0)$, be such that $q_{1}(\varrho)=q_{2}(\varrho)=0$, which reads $(-1)^{k} i\left(\sigma+i \hat{\tau}_{\sigma}\right)^{2}+a\left(x, \xi+i \hat{\tau}_{\xi}\right)=0$, for both $k=1$ and $k=2$, meaning that we have

$$
\left(\sigma+i \hat{\tau}_{\sigma}\right)^{2}=0, \quad a\left(x, \xi+i \hat{\tau}_{\xi}\right)=0 .
$$

In particular this implies $\sigma=0$ and $\hat{\tau}_{\sigma}=\tau \partial_{s} \varphi=0$. As here $\partial_{s} \varphi \neq 0$ we thus have $\sigma=\tau=0$. With $\tau=0$, we have $\hat{\tau}_{\xi}=0$, and we thus obtain $a(x, \xi)=0$, implying $\xi=0$ because of the ellipticity of $a(x, \xi)$.

Lemma 3.4. Let $L_{1}$ and $L_{2}$ be differential operators in $V$. Let $\varphi \in \mathscr{C}^{\infty}(Z)$ and $\operatorname{set} L_{k, \varphi}=e^{\tau \varphi} L_{k} e^{-\tau \varphi}$, $k=1,2$. Assume that $\operatorname{char}\left(L_{1, \varphi}\right) \cap \operatorname{char}\left(L_{2, \varphi}\right)=\emptyset$. Then the couple $\left(L_{1} L_{2}, \varphi\right)$ satisfies the subellipticity condition of Definition 2.4 in $\bar{V}$ if and only if both $\left(L_{k}, \varphi\right), k=1,2$, satisfy this property.

Proof. We denote by $\ell_{k}$, the principal symbols of $L_{k, \varphi}, k=1,2$, and $\ell=\ell_{1} \ell_{2}$ the principal symbol of $e^{\tau \varphi} L_{1} L_{2} e^{-\tau \varphi}$. We observe that

$$
\{\bar{\ell}, \ell\}=\left|\ell_{1}\right|^{2}\left\{\overline{\ell_{2}}, \ell_{2}\right\}+\left|\ell_{2}\right|^{2}\left\{\overline{\ell_{1}}, \ell_{1}\right\}+f\left|\ell_{1}\right|\left|\ell_{2}\right|,
$$

for some function $f$. If $(\ell, \varphi)$ satisfies the sub-ellipticity condition and if $\ell_{1}(\varrho)=0$, with $\varrho=$ $(z, \zeta, \tau) \in \bar{V} \times \mathbb{R}^{N} \times \mathbb{R}_{+}$, then $\ell_{2}(\varrho) \neq 0$ and $0<\{\bar{\ell}, \ell\}(\varrho) / i=\left|\ell_{2}\right|^{2}\left\{\bar{\ell}_{1}, \ell_{1}\right\} / i$, thus yielding the sub-ellipticity condition at $\varrho$ for $\ell_{1}$. The same argument applies for $\ell_{2}$.

Let us now assume that $\ell_{1}$ and $\ell_{2}$ both satisfy the sub-ellipticity condition. If $\ell(\varrho)=0$ then either $\ell_{1}(\varrho)=0$ or $\ell_{2}(\varrho)=0$. Let us assume that $\ell_{1}(\varrho)=0$. Then $\ell_{2}(\varrho) \neq 0$ and $\left\{\bar{\ell}_{1}, \ell_{1}\right\}(\varrho) / i>0$. We then have $\{\bar{\ell}, \ell\}(\varrho) / i=\left|\ell_{2}(\varrho)\right|^{2}\left\{\bar{\ell}_{1}, \ell_{1}\right\}(\varrho) / i>0$.

By Lemma 2.5, the couples $\left(P_{k}, \varphi\right)$ satisfy the sub-ellipticity condition in $\bar{V}$. From Lemmata 3.3 and 3.4 we deduce the following result.

Corollary 3.5. The couple $(P, \varphi)$ satisfies the sub-ellipticity condition of Definition 2.4 in $\bar{V}$.

3.4. Proof of the estimate at $\{s=0\}$. The proof of Theorem 3.1 uses Lemma 4.3 in [BL15].

Proof of Theorem 3.1. We denote by $a(\varrho)$ the principal symbol of $\left(P_{\varphi}+P_{\varphi}^{*}\right) / 2$ and by $b(\varrho)$ that of $\left(P_{\varphi}-P_{\varphi}^{*}\right) /(2 i)$. We have $a \in S_{\tau}^{4,0}$ and $b \in S_{\tau}^{3,1}$. We set $A=\mathrm{Op}(a)$ and $B=\mathrm{Op}(b)$ and

$$
Q_{a, b}(w)=2 \operatorname{Re}(A w, B w)_{+} .
$$

The sub-ellipticity of $(P, \varphi)$ given by Corollary 3.5 reads

$$
a(\varrho)=b(\varrho)=0 \Rightarrow\{a, b\}>0, \quad \varrho \in \bar{V} \times \mathbb{R}^{N} \times \mathbb{R}_{+} .
$$

With Lemma 4.3 in [BL15], we obtain, for some $C>0$ and $C^{\prime}>0$, for $\tau \geq 1$ chosen sufficiently large,

$$
C\|v\|_{4, \tau}^{2} \leq C^{\prime}\left(\|A v\|_{+}^{2}+\|B v\|_{+}^{2}+|\operatorname{tr}(v)|_{3,1 / 2, \tau}^{2}\right)+\tau\left(Q_{a, b}(v)-\operatorname{Re} \mathscr{B}_{a, b}(v)\right),
$$

where $\left|\mathscr{B}_{a, b}(v)\right| \lesssim|\operatorname{tr}(v)|_{3,1 / 2, \tau}^{2}$, for $v=w_{\mid Z}$, with $w \in \mathscr{C}_{c}^{\infty}\left(\mathbb{R}^{d} \times \mathbb{R}\right)$ and $\operatorname{supp}(w) \subset W$. We thus obtain

$$
\tau^{-1}\|v\|_{4, \tau}^{2} \lesssim\|(A+i B) v\|_{+}^{2}+|\operatorname{tr}(v)|_{3,1 / 2, \tau}^{2} .
$$

As we have $P_{\varphi}=A+i B \bmod \Psi_{\tau}^{3,0}$, by taking $\tau$ sufficiently large, with the usual semi-classical argument (3.1) we obtain

$$
\tau^{-1 / 2}\|v\|_{4, \tau} \lesssim\left\|P_{\varphi} v\right\|_{+}+|\operatorname{tr}(v)|_{3,1 / 2, \tau} .
$$

The conclusion of the proof is then classical. 
Proof of Corollary 3.2. Let $W^{\prime}$ be an open set of $\mathbb{R}^{N}$ such that $W \Subset W^{\prime} \Subset V$ and let $\chi, \tilde{\chi} \in \mathscr{C}_{c}^{\infty}\left(W^{\prime}\right)$ be such that $\chi \equiv 1$ in a neighborhood of $W$ and $\tilde{\chi} \equiv 1$ in a neighborhood of $\operatorname{supp}(\chi)$.

We may apply estimate (3.2), an equivalent form of the estimate of Theorem 3.1, to the function $\tau^{1 / 2} \chi(z) \Lambda_{\mathrm{T}, \tau}^{-1 / 2} v$, for $v=w_{\mid Z}$, with $w \in \mathscr{C}_{c}^{\infty}\left(\mathbb{R}^{d} \times \mathbb{R}\right)$ and $\operatorname{supp}(w) \subset W$. Observe that we have

$$
\chi(z) \Lambda_{\mathrm{T}, \tau}^{-1 / 2} v=\Lambda_{\mathrm{T}, \tau}^{-1 / 2} v+R_{0,-M} v, \quad P_{\varphi} \chi(z) \Lambda_{\mathrm{T}, \tau}^{-1 / 2} v=\tilde{\chi}(z) P_{\varphi} \Lambda_{\mathrm{T}, \tau}^{-1 / 2} v+R_{4,-M} v,
$$

because of the support of $v$, with $R_{0,-M} \in \Psi_{\tau}^{0,-M}$, and $R_{4,-M} \in \Psi_{\tau}^{4,-M}$, for any $M \in \mathbb{N}$.

Setting $\tilde{v}=\tau^{1 / 2} \Lambda_{\mathrm{T}, \tau}^{-1 / 2} v \in \mathscr{S}\left(\overline{\mathbb{R}_{+}^{N}}\right)$, we thus obtain, with (3.2),

$$
\tau^{-1 / 2}\|\tilde{v}\|_{4, \tau} \lesssim\left\|\tilde{\chi} P_{\varphi} \tilde{v}\right\|_{+}+|\operatorname{tr}(\tilde{v})|_{3,1 / 2, \tau}+\|v\|_{4,-M, \tau} .
$$

We then observe that we have

$$
\tau^{-1 / 2}\|\tilde{v}\|_{4, \tau}=\left\|\Lambda_{\mathrm{T}, \tau}^{-1 / 2} v\right\|_{4, \tau}=\|v\|_{4,-1 / 2, \tau} .
$$

We also have $|\operatorname{tr}(\tilde{v})|_{3,1 / 2, \tau}=\tau^{1 / 2}|\operatorname{tr}(v)|_{3,0, \tau}$, as $\left[D_{s}, \Lambda_{\mathrm{T}, \tau}^{r}\right]=0, r \in \mathbb{R}$. Next, as $\left[\tilde{\chi} P_{\varphi}, \Lambda_{\mathrm{T}, \tau}^{-1 / 2}\right] \in$ $\Psi_{\tau}^{4,-3 / 2}$, we have

$$
\left\|\tilde{\chi} P_{\varphi} \tilde{v}\right\|_{+} \lesssim \tau^{1 / 2}\left\|\Lambda_{\mathrm{T}, \tau}^{-1 / 2} \tilde{\chi} P_{\varphi} v\right\|_{+}+\tau^{1 / 2}\|v\|_{4,-3 / 2, \tau} \lesssim\left\|P_{\varphi} v\right\|_{+}+\tau^{1 / 2}\|v\|_{4,-3 / 2, \tau} .
$$

From (3.3), we thus obtain

$$
\|v\|_{4,-1 / 2, \tau} \lesssim\left\|P_{\varphi} v\right\|_{+}+\tau^{1 / 2}|\operatorname{tr}(\tilde{v})|_{3,0, \tau}+\tau^{1 / 2}\|v\|_{4,-3 / 2, \tau} .
$$

With the usual semi-classical argument (3.1) we conclude the proof, as $\|v\|_{4,-1 / 2, \tau} \gtrsim \tau^{1 / 2}\|v\|_{3, \tau}$.

4. Estimate at the boundary $\left(0, S_{0}\right) \times \partial \Omega$

4.1. A semi-classical calculus with three parameters. We set $\mathcal{W}=\mathbb{R}^{N} \times \mathbb{R}^{N}, N=d+1$, often referred to as phase-space. A typical element of $\mathcal{W}$ will be $X=(s, x, \sigma, \xi)$, with $s \in \mathbb{R}, x \in \mathbb{R}^{d}$, $\sigma \in \mathbb{R}$, and $\xi \in \mathbb{R}^{d}$. We also write $x=\left(x^{\prime}, x_{d}\right), x^{\prime} \in \mathbb{R}^{d-1}, x_{d} \in \mathbb{R}$, and accordingly $\xi=\left(\xi^{\prime}, \xi_{d}\right)$.

With $s$ and $x$ playing very similar rôle in the definition of the calculus, we set $z=(s, x) \in \mathbb{R}^{N}$, $z^{\prime}=\left(s, x^{\prime}\right) \in \mathbb{R}^{N-1}$, and $z_{N}=x_{d}$. We also set $\zeta=(\sigma, \xi) \in \mathbb{R}^{N}, \zeta^{\prime}=\left(\sigma, \xi^{\prime}\right) \in \mathbb{R}^{N-1}$, and $\zeta_{N}=\xi_{d}$.

In this section, we shall consider a weight function of the form

$$
\varphi_{\gamma, \varepsilon}(z)=e^{\gamma \psi_{\varepsilon}(z)}, \quad \psi_{\varepsilon}(z)=\psi\left(\varepsilon z^{\prime}, z_{N}\right)
$$

with $\gamma$ and $\varepsilon$ as parameters, satisfying $\gamma \geq 1, \varepsilon \in[0,1]$, and $\psi \in \mathscr{C}^{\infty}\left(\mathbb{R}^{N}\right)$. To define a proper pseudo-differential calculus, we assume the following properties of $\psi$ :

$$
\psi \geq C>0, \quad\left\|\psi^{(k)}\right\|_{L^{\infty}}<\infty, k \in \mathbb{N} .
$$

In particular, there exists $k>0$ such that

$$
\sup _{\mathbb{R}^{N}} \psi \leq(k+1) \inf _{\mathbb{R}^{N}} \psi
$$

4.1.1. A class of semi-classical symbols. We introduce the following class of tangential symbols depending on the variables $z \in \mathbb{R}^{N}, \zeta^{\prime} \in \mathbb{R}^{N-1}$ and $\hat{t} \in \mathbb{R}^{N}$. We set $\hat{\lambda}_{\mathrm{T}}^{2}=\left|\zeta^{\prime}\right|^{2}+|\hat{t}|^{2}$.

Definition 4.1. Let $m \in \mathbb{R}$. We say that $a\left(z, \zeta^{\prime}, \hat{t}\right) \in \mathscr{C}^{\infty}\left(\overline{\mathbb{R}_{+}^{N}} \times \mathbb{R}^{N-1} \times \mathbb{R}^{N}\right)$ belong to the class $S_{\mathrm{T}, \hat{t}}^{m}$ if, for all multi-indices $\alpha \in \mathbb{N}^{N}, \beta \in \mathbb{N}^{N-1}, \delta \in \mathbb{N}^{N}$, there exists $C_{\alpha, \beta, \delta}>0$ such that

$$
\left|\partial_{z}^{\alpha} \partial_{\zeta^{\prime}}^{\beta} \partial_{\hat{t}}^{\delta} a\left(z, \zeta^{\prime}, \hat{t}\right)\right| \leq C_{\alpha, \beta, \delta} \hat{\lambda}_{\mathrm{T}}^{m-|\beta|-|\delta|}, \quad\left(z, \zeta^{\prime}, \hat{t}\right) \in \overline{\mathbb{R}_{+}^{N}} \times \mathbb{R}^{N-1} \times \mathbb{R}^{N},|\hat{t}| \geq 1 .
$$

If $\Gamma$ is a conic open set of $\overline{\mathbb{R}_{+}^{N}} \times \mathbb{R}^{N-1} \times \mathbb{R}^{N}$, we say that $a \in S_{\mathrm{T}, \hat{t}}^{m}$ in $\Gamma$ if the above property holds for $\left(z, \zeta^{\prime}, \hat{t}\right) \in \Gamma$. 
Note that, as opposed to usual semi-classical symbols, we ask for some regularity with respect to the semi-classical parameter that is a vector of $\mathbb{R}^{N}$ here.

This class of symbols will not be used as such to define a class of pseudo-differential operators but rather to generate other classes of symbols and associated operators in a more refined semiclassical calculus that we present now.

4.1.2. Metrics. For $\tau_{*} \geq 2$, we set

$$
\begin{aligned}
& \mathcal{M}=\mathbb{R}^{N} \times \mathbb{R}^{N} \times\left[\tau_{*},+\infty\right) \times[1,+\infty) \times[0,1], \\
& \mathcal{M}_{\top}=\overline{\mathbb{R}_{+}^{N}} \times \mathbb{R}^{N-1} \times\left[\tau_{*},+\infty\right) \times[1,+\infty) \times[0,1] .
\end{aligned}
$$

We denote by $\varrho=(z, \zeta, \tau, \gamma, \varepsilon)$ a point in $\mathcal{M}$ and by $\varrho^{\prime}=\left(z, \zeta^{\prime}, \tau, \gamma, \varepsilon\right)$ a point in $\mathcal{M}_{\mathrm{T}}$.

We set $\tilde{\tau}=\tau \gamma \varphi_{\gamma, \varepsilon}(z) \in \mathbb{R}_{+}$. For simplicity, even though $\tilde{\tau}$ is independent of $\zeta^{\prime}$, we shall write $\tilde{\tau}=\tilde{\tau}\left(\varrho^{\prime}\right)$, when we wish to keep in mind that $\tilde{\tau}$ is not a simple parameter but rather a function. As $\psi>0, \tau \geq \tau_{*}$, and $\gamma \geq 1$,we note that we have $\tilde{\tau} \geq \tau_{*}$. We then set

$$
\lambda_{\tilde{\tau}}^{2}=\lambda_{\tilde{\tau}}^{2}(\varrho)=|\zeta|^{2}+\tilde{\tau}\left(\varrho^{\prime}\right)^{2}, \quad \lambda_{\mathrm{T}, \tilde{\tau}}^{2}=\lambda_{\mathrm{T}, \tilde{\tau}}^{2}\left(\varrho^{\prime}\right)=\left|\zeta^{\prime}\right|^{2}+\tilde{\tau}\left(\varrho^{\prime}\right)^{2} .
$$

The explicit dependences of $\lambda_{\tilde{\tau}}$ and $\lambda_{\mathrm{T}, \tilde{\tau}}$ upon $\varrho$ and $\varrho^{\prime}$ are now dropped to ease notation in this section. Similarly, we shall write $\varphi(z)$, or simply $\varphi$, in place of $\varphi_{\gamma, \varepsilon}(z)$.

We consider the following metric on phase-space $\mathcal{W}=\mathbb{R}^{N} \times \mathbb{R}^{N}$

$$
g=(1+\gamma \varepsilon)^{2}\left|d z^{\prime}\right|^{2}+\gamma^{2}\left|d z_{N}\right|^{2}+\lambda_{\tilde{\tau}}^{-2}|d \zeta|^{2},
$$

for $\tau \geq \tau_{*}, \gamma \geq 1$, and $\varepsilon \in[0,1]$. (Note that this metric is not to be confused with the Riemannian metric $g$ on $\Omega$.)

On the phase-space $\mathcal{W}^{\prime}=\mathbb{R}^{N} \times \mathbb{R}^{N-1}$ adapted to a tangential calculus, we consider the following metric:

$$
g_{\mathrm{T}}=(1+\gamma \varepsilon)^{2}\left|d z^{\prime}\right|^{2}+\gamma^{2}\left|d z_{N}\right|^{2}+\lambda_{\mathrm{T}, \tilde{\tau}}^{-2}\left|d \zeta^{\prime}\right|^{2},
$$

for $\tau \geq \tau_{*}, \gamma \geq 1$, and $\varepsilon \in[0,1]$.

The first result of this section shows that the metric $g$ on $\mathcal{W}$ defines a Weyl-Hörmander pseudodifferential calculus, and that both $\varphi$ and $\lambda_{\tilde{\tau}}$ have the properties to be used as proper order functions. For a presentation of the Weyl-Hörmander calculus we refer to [Ler10], [Hör85b, Sections 18.4-6] and [Hör79].

Proposition 4.2. The metric $g$ and the order functions $\varphi_{\gamma, \varepsilon}, \lambda_{\tilde{\tau}}$ are admissible, in the sense that, the following properties hold (uniformly with respect to the parameters $\tau, \gamma$, and $\varepsilon$ ):

(1) $g$ satisfies the uncertainty principle, that is $h_{g}^{-1}=\gamma^{-1} \lambda_{\tilde{\tau}} \geq 1$.

(2) $\varphi_{\gamma, \varepsilon}, \lambda_{\tilde{\tau}}$ and $g$ are slowly varying;

(3) $\varphi_{\gamma, \varepsilon}, \lambda_{\tilde{\tau}}$ and $g$ are temperate.

We refer to Appendix A.2.1 for a proof. Similarly, we have the following proposition.

Proposition 4.3. The metric $g_{\top}$ and the order functions $\varphi_{\gamma, \varepsilon}, \lambda_{\mathrm{T}, \tilde{\tau}}$ are admissible. For the tangential calculus we have $h_{g_{\top}}^{-1}=(1+\varepsilon \gamma)^{-1} \lambda_{\mathrm{T}, \tilde{\tau}} \geq 1$.

Note that the proof of the uncertainty principle uses that $\tau_{*} \geq 2$. The condition $\tau_{*} \geq 1$ would suffice if we chose $\psi \geq \ln (2)$. We preferred not to add this technical condition on the weight function $\psi$.

Consequently, $\tilde{\tau}\left(\varrho^{\prime}\right)$ is also an admissible order function for both calculi. 
4.1.3. Symbols. Let $a(\varrho) \in \mathscr{C}^{\infty}\left(\mathbb{R}^{N} \times \mathbb{R}^{N}\right)$, with $\tau, \gamma$, and $\varepsilon$ acting as parameters, and $m, r \in \mathbb{R}$, be such that for all multi-indices $\alpha, \beta \in \mathbb{N}^{N}$, with $\alpha=\left(\alpha^{\prime}, \alpha_{N}\right)$, we have

$$
\left|\partial_{z}^{\alpha} \partial_{\zeta}^{\beta} a(\varrho)\right| \leq C_{\alpha, \beta} \gamma^{\left|\alpha_{N}\right|}(1+\varepsilon \gamma)^{\left|\alpha^{\prime}\right|} \tilde{\tau}^{r} \lambda_{\tilde{\tau}}^{m-|\beta|}, \quad \varrho \in \mathcal{M} .
$$

With the notation of [Hör85b, Sections 18.4-18.6] we then have $a(\varrho) \in S\left(\tilde{\tau}^{r} \lambda_{\tilde{\tau}}^{m}, g\right)$.

Similarly, let $a\left(\varrho^{\prime}\right) \in \mathscr{C}^{\infty}\left(\overline{\mathbb{R}_{+}^{N}} \times \mathbb{R}^{N-1}\right)$, with $\tau, \gamma$, and $\varepsilon$ acting as parameters, and $m \in \mathbb{R}$. If for all multi-indices $\alpha=\left(\alpha^{\prime}, \alpha_{N}\right) \in \mathbb{N}^{N}, \beta^{\prime} \in \mathbb{N}^{N-1}$, we have

$$
\left|\partial_{z}^{\alpha} \partial_{\zeta^{\prime}}^{\beta^{\prime}} a\left(\varrho^{\prime}\right)\right| \leq C_{\alpha, \beta^{\prime}} \gamma^{\left|\alpha_{N}\right|}(1+\varepsilon \gamma)^{\left|\alpha^{\prime}\right|} \tilde{\tau}^{r} \lambda_{\mathrm{T}, \tilde{\tau}}^{m-\left|\beta^{\prime}\right|}, \quad \varrho^{\prime} \in \mathcal{M}_{\mathrm{T}},
$$

we then write $a\left(\varrho^{\prime}\right) \in S\left(\tilde{\tau}^{r} \lambda_{\mathrm{T}, \tilde{\tau}}^{m}, g_{\mathrm{T}}\right)$. Observe that $S\left(\tilde{\tau}^{r} \lambda_{\mathrm{T}, \tilde{\tau}}^{m}, g_{\mathrm{T}}\right) \subset S\left(\lambda_{\mathrm{T}, \tilde{\tau}}^{r+m}, g_{\mathrm{T}}\right)$.

The principal symbol associated with $a\left(\varrho^{\prime}\right) \in S\left(\tilde{\tau}^{r} \lambda_{\mathrm{T}, \tilde{\tau}}^{m}, g_{\mathrm{T}}\right)$ is given by its equivalence class in $S\left(\tilde{\tau}^{r} \lambda_{\mathrm{T}, \tilde{\tau}}^{m}, g_{\mathrm{T}}\right) / S\left((1+\varepsilon \gamma) \tilde{\tau}^{r} \lambda_{\mathrm{T}, \tilde{\tau}}^{m-1}, g_{\mathrm{T}}\right)$. We denote this principal part by $\sigma(a)$. Often, an homogeneous representative can be selected and the principal part is then identified with this particular representative of the equivalence class. (Conic sets and homogeneous symbols are precisely defined in Section 4.1.5 below.)

We define the following class of symbols, that are polynomial with respect to $\xi_{N}$,

$$
S_{\tilde{\tau}}^{m, m^{\prime}}=\sum_{j=0}^{m} S\left(\lambda_{\mathrm{T}, \tilde{\tau}}^{m+m^{\prime}-j}, g_{\mathrm{T}}\right) \zeta_{N}^{j} .
$$

For $a(\varrho) \in S_{\tilde{\tau}}^{m, m^{\prime}}$, with $a(\varrho)=\sum_{j=0}^{m} a_{j}\left(\varrho^{\prime}\right) \zeta_{N}^{j}$, with $a_{j}\left(\varrho^{\prime}\right) \in S\left(\lambda_{\mathrm{T}, \tilde{\tau}}^{m+m^{\prime}-j}, g_{\mathrm{T}}\right)$, we denote its principal part by $\sigma(a)(\varrho)=\sum_{j=0}^{m} \sigma\left(a_{j}\right)\left(\varrho^{\prime}\right) \zeta_{N}^{j}$.

For this calculus with parameters to make sense, it is important to check that $\lambda_{\tilde{\tau}} \in S\left(\lambda_{\tilde{\tau}}, g\right)$ and $\lambda_{\top, \tilde{\tau}} \in S\left(\lambda_{\top, \tilde{\tau}}, g_{\top}\right)$ and $\tilde{\tau} \in S(\tilde{\tau}, g) \cap S\left(\tilde{\tau}, g_{\top}\right)$. In fact, the latter property implies the first two.

Lemma 4.4. We have $\tilde{\tau}=\tau \gamma \varphi_{\gamma, \varepsilon} \in S(\tilde{\tau}, g) \cap S\left(\tilde{\tau}, g_{\top}\right)$.

We refer to Section A.2.2 for a proof.

4.1.4. A semi-classical cotangent vector. We set $\hat{\tau}=\tau d_{z} \varphi_{\gamma, \varepsilon}(z)=\tau \gamma \varphi_{\gamma, \varepsilon}(z) d_{z} \psi_{\varepsilon}(z)=\tilde{\tau}\left(\varrho^{\prime}\right) d_{z} \psi_{\varepsilon}(z) \in$ $\mathbb{R}^{N}$. As for $\tilde{\tau}$, we shall write $\hat{\tau}=\hat{\tau}\left(\varrho^{\prime}\right)$, when we wish to keep in mind that $\hat{\tau}$ is not a constant cotangent vector. Note that $\hat{\tau}=\left(\hat{\tau}^{\prime}, \hat{\tau}_{N}\right)$ with

$$
\hat{\tau}^{\prime}\left(\varrho^{\prime}\right)=\tilde{\tau}\left(y^{\prime}\right) d_{z^{\prime}} \psi_{\varepsilon}(z)=\varepsilon \tilde{\tau}\left(y^{\prime}\right) d_{z^{\prime}} \psi\left(\varepsilon z^{\prime}, z_{N}\right), \quad \hat{\tau}_{N}\left(\varrho^{\prime}\right)=\tilde{\tau}\left(y^{\prime}\right) \partial_{z_{N}} \psi\left(\varepsilon z^{\prime}, z_{N}\right) .
$$

As $d_{z^{\prime}} \psi_{\varepsilon} \in S\left(\varepsilon, g_{\mathrm{T}}\right)$ and $\partial_{z_{N}} \psi_{\varepsilon} \in S\left(1, g_{\mathrm{T}}\right)$, we have the following result.

Lemma 4.5. We have $\hat{\tau}^{\prime} \in S(\varepsilon \tilde{\tau}, g)^{N-1} \cap S\left(\varepsilon \tilde{\tau}, g_{\mathrm{T}}\right)^{N-1}$ and $\hat{\tau}_{N} \in S(\tilde{\tau}, g) \cap S\left(\tilde{\tau}, g_{\mathrm{T}}\right)$.

For later use, we also introduce the following notation:

$$
\begin{aligned}
& \hat{\tau}_{\sigma}=\hat{\tau}_{\sigma}\left(\varrho^{\prime}\right)=\tau \partial_{s} \varphi_{\gamma, \varepsilon}(z) \in \mathbb{R}, \quad \hat{\tau}_{\xi}=\hat{\tau}_{\xi}\left(\varrho^{\prime}\right)=\tau d_{x} \varphi_{\gamma, \varepsilon}(z) \in \mathbb{R}^{N-1}=\mathbb{R}^{d}, \\
& \hat{\tau}_{\xi_{d}}=\hat{\tau}_{\xi_{d}}\left(\varrho^{\prime}\right)=\tau \partial_{x_{d}} \varphi_{\gamma, \varepsilon}(z) \in \mathbb{R}, \quad \hat{\tau}_{\xi^{\prime}}=\hat{\tau}_{\xi^{\prime}}\left(\varrho^{\prime}\right)=\tau d_{x^{\prime}} \varphi_{\gamma, \varepsilon}(z) \in \mathbb{R}^{N-2}=\mathbb{R}^{d-1} .
\end{aligned}
$$

We then have

$$
\hat{\tau}=\left(\hat{\tau}_{\sigma}, \hat{\tau}_{\xi}\right)=\left(\hat{\tau}_{\sigma}, \hat{\tau}_{\xi^{\prime}}, \hat{\tau}_{\xi_{d}}\right), \quad \hat{\tau}^{\prime}=\left(\hat{\tau}_{\sigma}, \hat{\tau}_{\xi^{\prime}}\right), \quad \hat{\tau}_{N}=\hat{\tau}_{\xi_{d}} .
$$

Even thought the following lemma is very elementary, we state it for futur reference.

Lemma 4.6. Let $V$ be an open set of $\mathbb{R}^{N}$ such that $\partial_{x_{d}} \psi(z) \geq C>0$ for $z \in V$. Then, we have

$$
|\hat{\tau}| \asymp \hat{\tau}_{\xi_{d}} \asymp \tilde{\tau}, \quad z \in V .
$$

Proof. As $\left\|\psi^{\prime}\right\|_{\infty} \leq C$, if $\partial_{x_{d}} \psi \geq C>0$ for $z \in V \subset \mathbb{R}^{N}$, then we have $|\hat{\tau}| \lesssim \tilde{\tau} \lesssim \hat{\tau}_{\xi_{d}}$ and thus the result. 
4.1.5. Conic sets and homogeneity. We recall that a set $\Gamma \subset \overline{\mathbb{R}_{+}^{N}} \times \mathbb{R}^{N-1} \times \mathbb{R}^{N}$ is said to be conic if $\left(z, \zeta^{\prime}, \hat{t}\right) \in \Gamma$ implies that $\left(z, v \zeta^{\prime}, v \hat{t}\right) \in \Gamma$ for all $v>0$.

We introduce the map

$$
\begin{aligned}
\kappa: \mathcal{M}_{\mathrm{T}} & \rightarrow \overline{\mathbb{R}_{+}^{N}} \times \mathbb{R}^{N-1} \times \mathbb{R}^{N}, \\
\varrho^{\prime}=\left(z, \zeta^{\prime}, \tau, \gamma, \varepsilon\right) & \mapsto\left(z, \zeta^{\prime}, \hat{\tau}\left(\varrho^{\prime}\right)\right) .
\end{aligned}
$$

Throughout Section 4 and Appendix B, we shall use the following terminology.

Definition 4.7. An open subset $\mathscr{U}$ of $\mathcal{M}_{\top}$ is said to be conic if $\Gamma=\kappa(\mathscr{U})$ is conic in $\overline{\mathbb{R}_{+}^{N}} \times \mathbb{R}^{N-1} \times \mathbb{R}^{N}$.

A function $f: \mathscr{U} \rightarrow E, E$ a vector space, is said to be homogeneous of degree $m$ if $f$ takes the form $f=g \circ \kappa$ with $g: \overline{\mathbb{R}_{+}^{N}} \times \mathbb{R}^{N-1} \times \mathbb{R}^{N} \rightarrow E$ such that $g(z, v \zeta, v \hat{t})=v^{m} g(z, \zeta, \hat{t})$, for $v>0$.

In other words, conic sets and homogeneity are to be understood with respect to the variables $(z, \zeta, \hat{\tau})$ instead of the variables $(z, \zeta, \tau, \gamma, \varepsilon)$, where, as above, $\hat{\tau}=\tau d_{z} \varphi_{\gamma, \varepsilon}(z)=\tau \gamma \varphi_{\gamma, \varepsilon}(z) d_{z} \psi_{\varepsilon}(z)$.

If $\mathscr{U}$ is a conic open subset of $\mathcal{M}_{\top}$ we shall say that $a \in S\left(\tilde{\tau}^{r} \lambda_{\mathrm{T}, \tilde{\tau}}^{m}, g_{\top}\right)$ in $\mathscr{U}$ if property (4.6) holds in $\mathscr{U}$, with a similar terminology for symbols that satisfy the defining property of $S_{\tilde{\tau}}^{m, m^{\prime}}$ in $\mathscr{U}$.

In what follows, the following lemma will be used for instance, to generate cutoff functions. It will also be used to obtain symbols with the adapted homogeneity with respect to $\zeta^{\prime}$ and $\hat{\tau}$. We refer to Section A.2.3 for a proof.

Lemma 4.8. Let $\mathscr{U}$ be a conic open subset of $\mathcal{M}_{\top}$ and set $\Gamma=\kappa(\mathscr{U})$. Assume also that $|\hat{\tau}| \asymp \tilde{\tau}$ in $\mathscr{U}$. Let $m \in \mathbb{R}$ and $\hat{a}\left(z, \zeta^{\prime}, \hat{t}\right) \in S_{\mathrm{T}, \hat{t}}^{m}$ in $\Gamma$ (as given in Definition 4.1). We then have a $\left(\varrho^{\prime}\right)=$ $\hat{a} \circ \kappa\left(\varrho^{\prime}\right) \in S\left(\lambda_{\mathrm{T}, \tilde{\tau}}^{m}, g_{\mathrm{T}}\right)$ in $\mathscr{U}$. In fact, if $\hat{a}$ is polynomial in $\left(\zeta^{\prime}, \hat{t}\right)$ the assumption $|\hat{\tau}| \asymp \tilde{\tau}$ in $\mathscr{U}$ is not needed.

The following lemma is elementary.

Lemma 4.9. Let $\mathscr{U}$ be a conic open subset of $\mathcal{M}_{\mathrm{T}}$ and let $a \in S\left(\tilde{\tau}^{r} \lambda_{\mathrm{T}, \tilde{\tau}}^{m}, g_{\mathrm{T}}\right)$ in $\mathscr{U}$. Let $\chi \in S\left(1, g_{\mathrm{T}}\right)$ in $\mathcal{M}_{\mathrm{T}}$, with $\operatorname{supp}(\chi) \subset \mathscr{U}$. Then, $\chi a \in S\left(\tilde{\tau}^{r} \lambda_{\mathrm{T}, \tilde{\tau}}^{m}, g_{\mathrm{T}}\right)$ in $\mathcal{M}_{\mathrm{T}}$.

4.1.6. Operators and Sobolev bounds. For $a \in S\left(\tilde{\tau}^{r} \lambda_{\tilde{\tau}}^{m}, g\right)$ we define the following pseudo-differential operator in $\mathbb{R}^{N}$ :

$$
\mathrm{Op}(a) u(z)=(2 \pi)^{-N} \int_{\mathbb{R}^{N}} e^{i z \cdot \zeta} a(z, \zeta, \tau, \gamma, \varepsilon) \hat{u}(\zeta) d \zeta, \quad u \in \mathscr{S}\left(\mathbb{R}^{N}\right),
$$

where $\hat{u}$ is the Fourier transform of $u$. In the sense of oscillatory integrals, we have

$$
\mathrm{Op}(a) u(z)=(2 \pi)^{-N} \iint_{\mathbb{R}^{2 N}} e^{i(z-y) \cdot \zeta} a(z, \zeta, \tau, \gamma, \varepsilon) u(y) d \zeta d y .
$$

The associated class of pseudo-differential operators is denoted by $\Psi\left(\tilde{\tau}^{r} \lambda_{\tilde{\tau}}^{m}, g\right)$. If $a$ is polynomial in the variables $\zeta$ and $\hat{\tau}\left(\varrho^{\prime}\right)=\tilde{\tau} d_{z} \psi_{\varepsilon}(z)$, we then write $\operatorname{Op}(a) \in \mathscr{D}\left(\tilde{\tau}^{r} \lambda_{\tilde{\tau}}^{m}, g\right)$.

Tangential operators are defined similarly. For $a \in S\left(\tilde{\tau}^{r} \lambda_{\mathrm{T}, \tilde{\tau}}^{m}, g_{\mathrm{T}}\right)$ we set

$$
\mathrm{Op}_{\mathrm{T}}(a) u(z)=(2 \pi)^{-(N-1)} \iint_{\mathbb{R}^{2 N-2}} e^{i\left(z^{\prime}-y^{\prime}\right) \cdot \zeta^{\prime}} a\left(z, \zeta^{\prime}, \tau, \gamma, \varepsilon\right) u\left(y^{\prime}, z_{N}\right) d \zeta^{\prime} d y^{\prime},
$$

for $u \in \mathscr{S}\left(\overline{\mathbb{R}_{+}^{N}}\right)$, where $z \in \overline{\mathbb{R}_{+}^{N}}$. We write $A=\operatorname{Op}_{\mathrm{T}}(a) \in \Psi\left(\tilde{\tau}^{r} \lambda_{\mathrm{T}, \tilde{\tau}}^{m}, g_{\mathrm{T}}\right)$. We set $\Lambda_{\mathrm{T}, \tilde{\tau}}^{m}=\operatorname{Op}_{\mathrm{T}}\left(\lambda_{\mathrm{T}, \tilde{\tau}}^{m}\right)$. 
We also introduce the following class of operators that act as differential operators in the $z_{N}$ variable and as tangential pseudo-differential operators in the $z^{\prime}$ variables:

$$
\Psi_{\tilde{\tau}}^{m, r}=\sum_{j=0}^{m} \Psi\left(\lambda_{\mathrm{T}, \tilde{\tau}}^{m+r-j}, g_{\mathrm{T}}\right) D_{z_{N}}^{j}, \quad m \in \mathbb{N}, r \in \mathbb{R}
$$

that is, $\mathrm{Op}(a) \in \Psi_{\tilde{\tau}}^{m, r}$ if $a \in S_{\tilde{\tau}}^{m, r}$. Operators of this class can be applied to functions that are only defined on the half-space $\left\{z_{N} \geq 0\right\}$.

At places, it will be handy to use the Weyl quantization for tangential operators, namely with $a \in S\left(\tilde{\tau}^{r} \lambda_{\mathrm{T}, \tilde{\tau}}^{m}, g_{\mathrm{T}}\right)$ we define

$$
\mathrm{Op}_{\mathrm{T}}{ }^{w}(a) u(z)=(2 \pi)^{-(N-1)} \iint_{\mathbb{R}^{2 N-2}} e^{i\left(z^{\prime}-y^{\prime}\right) \cdot \zeta^{\prime}} a\left(\left(z^{\prime}+y^{\prime}\right) / 2, z_{N}, \zeta^{\prime}, \tau, \gamma, \varepsilon\right) u\left(y^{\prime}, z_{N}\right) d \zeta^{\prime} d y^{\prime} .
$$

This quantification is often advantageous as $\mathrm{Op}_{T}^{w}(a)^{*}=\mathrm{Op}_{\mathrm{T}}{ }^{w}(\bar{a})$, and thus, for the symbol $a$ real, the operator $\mathrm{Op}_{\mathrm{T}}{ }^{w}(a)$ is (formally) selfadjoint. Note that $\mathrm{Op}_{\mathrm{T}}(a)-\mathrm{Op}_{\mathrm{T}}{ }^{w}(a) \in(1+\varepsilon \gamma) \Psi\left(\tilde{\tau}^{r} \lambda_{\mathrm{T}, \tilde{\tau}}^{m-1}, g_{\mathrm{T}}\right)$.

We now present some Sobolev-bound type result that we shall use in what follows. We use the following notation

$$
\|.\|_{+}=\|\cdot\|_{L^{2}\left(\overline{\mathbb{R}_{+}^{N}}\right)}, \quad(., .)_{+}=(., .){ }_{L^{2}\left(\overline{\mathbb{R}_{+}^{N}}\right)},
$$

for the $L^{2}$-norm on the half space $\overline{\mathbb{R}_{+}^{N}}$ and the associated scalar product.

We have the following lemma whose proof is similar to that of Lemma 2.7 in [Le 15].

Lemma 4.10. Let $r, m \in \mathbb{R}$ and $a \in S\left(\tilde{\tau}^{r} \lambda_{\mathrm{T}, \tilde{\tau}}^{m}, g_{\mathrm{T}}\right)$. There exists $C>0$ such that, for $\tau$ sufficiently large,

$$
\left|\left(\mathrm{Op}_{\mathrm{T}}(a) u, v\right)_{+}\right| \leq C\left\|\mathrm{Op}_{\mathrm{T}}\left(\tilde{\tau}^{r^{\prime}} \lambda_{\mathrm{T}, \tilde{\tau}}^{m^{\prime}}\right) u\right\|_{+}\left\|\mathrm{Op}_{\mathrm{T}}\left(\tilde{\tau}^{r^{\prime \prime}} \lambda_{\mathrm{T}, \tilde{\tau}}^{m^{\prime \prime}}\right) v\right\|_{+}, \quad u, v \in \mathscr{S}\left(\overline{\mathbb{R}_{+}^{N}}\right) .
$$

for $r=r^{\prime}+r^{\prime \prime}, m=m^{\prime}+m^{\prime \prime}$, with $r^{\prime}, r^{\prime \prime} \in \mathbb{R}, m^{\prime}, m^{\prime \prime} \in \mathbb{R}$.

This contains the estimate

$$
\left\|\mathrm{Op}_{\mathrm{T}}\left(\tilde{\tau}^{r^{\prime}} \lambda_{\mathrm{T}, \tilde{\tau}}^{m^{\prime}}\right) \mathrm{Op}_{\mathrm{T}}(a) u\right\|_{+} \leq C\left\|\mathrm{Op}_{\mathrm{T}}\left(\tilde{\tau}^{r+r^{\prime}} \lambda_{\mathrm{T}, \tilde{\tau}}^{m+m^{\prime}}\right) u\right\|_{+}, \quad u \in \mathscr{S}\left(\overline{\mathbb{R}_{+}^{N}}\right),
$$

for $r, m^{\prime} \in \mathbb{R}$. The proof of Lemma 4.10 relies in the fact that, for $r, m \in \mathbb{R}$,

$$
\mathrm{Op}_{\mathrm{T}}\left(\tilde{\tau}^{r} \lambda_{\mathrm{T}, \tilde{\tau}}^{m}\right) \mathrm{Op}_{\mathrm{T}}\left(\tilde{\tau}^{-r} \lambda_{\mathrm{T}, \tilde{\tau}}^{-m}\right)=\mathrm{Id}+R_{1},
$$

with $R_{1} \in(1+\varepsilon \gamma) \Psi\left(\lambda_{\mathrm{T}, \tilde{\tau}}^{-1}, g_{\mathrm{T}}\right)$ and $\left\|R_{1}\right\|_{L^{2} \rightarrow L^{2}} \ll 1$ for $\tau$ large.

Note also that we have the following result (see Section A.2.4 for a proof).

Lemma 4.11. We have

$$
\left\|\mathrm{Op}_{\mathrm{T}}\left(\tilde{\tau}^{r} \lambda_{\mathrm{T}, \tilde{\tau}}^{m}\right) u\right\|_{+} \asymp\left\|\mathrm{Op}_{\mathrm{T}}\left(\lambda_{\mathrm{T}, \tilde{\tau}}^{m}\right) \tilde{\tau}^{r} u\right\|_{+}, \quad u \in \mathscr{S}\left(\overline{\mathbb{R}_{+}^{N}}\right),
$$

and

$$
\left|\mathrm{Op}_{\mathrm{T}}\left(\tilde{\tau}^{r} \lambda_{\mathrm{T}, \tilde{\tau}}^{m}\right) u_{z_{N}=0^{+}}\right|_{L^{2}\left(\mathbb{R}^{N-1}\right)} \asymp\left|\mathrm{Op}_{\mathrm{T}}\left(\lambda_{\mathrm{T}, \tilde{\tau}}^{m}\right) \tilde{\tau}^{r} u_{z_{N}=0^{+}}\right|_{L^{2}\left(\mathbb{R}^{N-1}\right)}, \quad u \in \mathscr{S}\left(\mathbb{R}^{N-1}\right),
$$

for $\tau$ chosen sufficiently large.

We define the following semi-classical Sobolev norms

$$
\begin{gathered}
|u|_{m, \tilde{\tau}}=\left|\Lambda_{\mathrm{T}, \tilde{\tau}}^{m} u_{\mid z_{N}=0^{+}}\right|_{L^{2}\left(\mathbb{R}^{N-1}\right)}, \quad m \in \mathbb{R}, \quad u \in \mathscr{S}\left(\mathbb{R}^{N-1}\right), \\
\|u\|_{m, \tilde{\tau}} \asymp \sum_{j=0}^{m}\left\|\Lambda_{\mathrm{T}, \tilde{\tau}}^{m-j} D_{z_{N}}^{j} u\right\|_{+}, \quad m \in \mathbb{N}, \quad u \in \mathscr{S}\left(\overline{\mathbb{R}_{+}^{N}}\right) .
\end{gathered}
$$


We also set, for $m \in \mathbb{N}$ and $m^{\prime} \in \mathbb{R}$,

$$
\|u\|_{m, m^{\prime}, \tilde{\tau}} \asymp \sum_{j=0}^{m}\left\|\Lambda_{\mathrm{T}, \tilde{\tau}}^{m-j+m^{\prime}} D_{z_{N}}^{j} u\right\|_{+}, \quad u \in \mathscr{S}\left(\overline{\mathbb{R}_{+}^{N}}\right) .
$$

At the boundary $\left\{z_{N}=0\right\}$ we define the following norms, for $m \in \mathbb{N}$ and $m^{\prime} \in \mathbb{R}$,

$$
\left.|\operatorname{tr}(u)|_{m, m^{\prime}, \tilde{\tau}} \asymp \sum_{j=0}^{m} \mid \Lambda_{\mathrm{T}, \tilde{\tau}}^{m-j+m^{\prime}} D_{z_{N}}^{j} u_{z_{N}=0^{+}}\right)\left.\right|_{L^{2}\left(\mathbb{R}^{N-1}\right)}, \quad u \in \mathscr{S}\left(\overline{\mathbb{R}_{+}^{N}}\right) .
$$

The following argument will be used on many occasions in what follows, for $r, r^{\prime}, m \in \mathbb{R}$, and $\ell>0$,

$$
\gamma^{r}\left\|\tilde{\tau}^{r^{\prime}} w\right\|_{m, \tilde{\tau}} \ll\left\|\tilde{\tau}^{r^{\prime}+\ell} w\right\|_{m, \tilde{\tau}} \lesssim\left\|\tilde{\tau}^{r^{\prime}} w\right\|_{m+\ell, \tilde{\tau}},
$$

for $\tau$ chosen sufficiently large, as $\gamma^{r} \lesssim \varphi_{\gamma, \varepsilon}=\exp \left(\gamma \psi_{\varepsilon}\right)$ since $\psi_{\varepsilon} \geq C>0$. We have similar such inequalities for the other norms introduced above.

With the above results we deduce the following two propositions.

Proposition 4.12. Let $r, m \in \mathbb{R}$, and $a \in S\left(\tilde{\tau}^{r} \lambda_{\mathrm{T}, \tilde{\tau}}^{m}, g_{\top}\right)$. Then, for $r^{\prime}, m^{\prime} \in \mathbb{R}$, there exists $C>0$ such that

$$
\left|\tilde{\tau}^{r^{\prime}} \operatorname{Op}_{\mathrm{T}}(a) u_{z_{N}=0^{+}}\right|_{m^{\prime}, \tilde{\tau}} \leq C\left|\tilde{\tau}^{r+r^{\prime}} u_{Z_{N}=0^{+}}\right|_{m+m^{\prime}, \tilde{\tau}}, \quad u \in \mathscr{S}\left(\overline{\mathbb{R}_{+}^{N}}\right),
$$

for $\tau$ sufficiently large.

Proposition 4.13. Let $r, m^{\prime} \in \mathbb{R}, m \in \mathbb{N}$, and $a \in \tilde{\tau}^{r} S_{\tilde{\tau}}^{m, m^{\prime}}$. Then, for $r^{\prime}, m^{\prime \prime \prime} \in \mathbb{R}$ and $m^{\prime \prime} \in \mathbb{N}$, there exists $C>0$ such that

$$
\left\|\tilde{\tau}^{r^{\prime}} \mathrm{Op}(a) u\right\|_{m^{\prime \prime}, m^{\prime \prime \prime}, \tilde{\tau}} \leq C\left\|\tilde{\tau}^{r+r^{\prime}} u\right\|_{m+m^{\prime \prime}, m^{\prime}+m^{\prime \prime \prime}, \tilde{\tau}}, \quad u \in \mathscr{S}\left(\overline{\mathbb{R}_{+}^{N}}\right),
$$

for $\tau$ sufficiently large.

Similarly to Lemma 4.11, we have the following equivalences for norms.

Lemma 4.14. Let $m \in \mathbb{N}$ and $r, m^{\prime} \in \mathbb{R}$. We have, for $\tau$ chosen sufficiently large,

$$
\left\|\tilde{\tau}^{r} w\right\|_{m, m^{\prime}, \tilde{\tau}} \asymp \sum_{j=0}^{m}\left\|D_{z_{N}}^{j}\left(\tilde{\tau}^{r} w\right)\right\|_{0, m+m^{\prime}-j, \tilde{\tau}} \asymp \sum_{j=0}^{m}\left\|\tilde{\tau}_{j}^{r^{\prime}} \Lambda_{\mathrm{T}, \tilde{\tau}}^{m_{j}^{\prime \prime}} D_{z_{N}}^{j}\left(\tilde{\tau}_{j}^{r_{j}^{\prime \prime}} \Lambda_{\mathrm{T}, \tilde{\tau}}^{m_{j}^{\prime \prime \prime}} w\right)\right\|_{+},
$$

where $r=r_{j}^{\prime}+r_{j}^{\prime \prime}$, and $m+m^{\prime}-j=m_{j}^{\prime \prime}+m_{j}^{\prime \prime \prime}$, with $r_{j}^{\prime}, r_{j}^{\prime \prime} \in \mathbb{R}$ and $m_{j}^{\prime \prime}, m_{j}^{\prime \prime \prime} \in \mathbb{R}, j=1, \ldots, m$. Similarly, we have

$$
\left|\operatorname{tr}\left(\tilde{\tau}^{r} w\right)\right|_{m, m^{\prime}, \tilde{\tau}} \asymp \sum_{j=0}^{m}\left|D_{z_{N}}^{j}\left(\tilde{\tau}^{r} w\right)_{z_{N}=0^{+}}\right|_{m+m^{\prime}-j, \tilde{\tau}} \asymp \sum_{j=0}^{m}\left|\tilde{\tau}^{r_{j}^{\prime}} \Lambda_{\mathrm{T}, \tilde{\tau}}^{m_{j}^{\prime \prime}}\left(D_{z_{N}}^{j}\left(\tilde{\tau}_{j}^{r_{j}^{\prime \prime}} \Lambda_{\mathrm{T}, \tilde{\tau}}^{m_{j}^{\prime \prime \prime}} w\right)\right)_{z_{N}=0^{+}}\right|_{L^{2}\left(\mathbb{R}^{n-1}\right)} .
$$

See Section A.2.5 for a proof.

Proposition 4.15 (local tangential Gårding inequality). Let $W_{0}, W_{1}$ be two open sets of $\mathbb{R}^{N}$, with $W_{0} \Subset W_{1}$. Let $a\left(\varrho^{\prime}\right) \in S\left(\tilde{\tau}^{r} \lambda_{\mathrm{T}, \tilde{\tau}}^{m}, g_{\mathrm{T}}\right)$, with principal part $a_{r, m}$. If there exist $C>0$ and $R>0$ such that

$$
\operatorname{Re} a_{r, m}\left(\varrho^{\prime}\right) \geq C \tilde{\tau}^{r} \lambda_{\mathrm{T}, \tilde{\tau}}^{m}, \quad z \in W_{1}, \quad \zeta^{\prime} \in \mathbb{R}^{N-1}, \quad \tau \geq \tau_{*}, \quad \lambda_{\mathrm{T}, \tilde{\tau}} \geq R,
$$

then for any $0<C^{\prime}<C$ there exists $\tau_{1} \geq \tau_{*}$ such that

$$
\operatorname{Re}\left(\mathrm{Op}_{\mathrm{T}}(a) u, u\right)_{+} \geq C^{\prime}\left\|\tilde{\tau}^{r / 2} u\right\|_{0, m / 2, \tilde{\tau}}^{2}, \quad \tau \geq \tau_{1} .
$$

for $u=w_{\mid Z}$, with $w \in \mathscr{C}_{c}^{\infty}\left(\left(0, S_{0}\right) \times \mathbb{R}^{d}\right)$ and $\operatorname{supp}(w) \subset W_{0}$.

In many occurrences, we shall use the following microlocal version of the Gårding inequality. 
Proposition 4.16 (microlocal tangential Gårding inequality). Let $\mathscr{U} \subset \mathcal{M}_{\mathrm{T}}$ be a conic open set. Let also $\chi\left(\varrho^{\prime}\right) \in S\left(1, g_{\mathrm{T}}\right)$ be homogeneous of degree zero and such that $\operatorname{supp}(\chi) \subset \mathscr{U}$. Let $r, m \in \mathbb{R}$ and $a\left(\varrho^{\prime}\right) \in S\left(\tilde{\tau}^{r} \lambda_{\mathrm{T}, \tilde{\tau}}^{m}, g_{\mathrm{T}}\right)$, with principal part $a_{r, m}$. If there exist $C>0$ and $R>0$ such that

$$
\operatorname{Re} a_{r, m}\left(\varrho^{\prime}\right) \geq C \tilde{\tau}^{r} \lambda_{\mathrm{T}, \tilde{\tau}}^{m}, \quad \varrho^{\prime} \in \mathscr{U}, \quad \tau \geq \tau_{*}, \quad \lambda_{\mathrm{T}, \tilde{\tau}} \geq R,
$$

then for any $0<C^{\prime}<C, M \in \mathbb{N}$, there exist $C_{M}$ and $\tau_{0} \geq \tau_{*}$ such that

$$
\operatorname{Re}\left(\mathrm{Op}_{\mathrm{T}}(a) \mathrm{Op}_{\mathrm{T}}(\chi) u, \mathrm{Op}_{\mathrm{T}}(\chi) u\right)_{+} \geq C^{\prime}\left\|\tilde{\tau}^{r / 2} \mathrm{Op}_{\mathrm{T}}(\chi) u\right\|_{0, m / 2, \tilde{\tau}}^{2}-C_{M}\|u\|_{0,-M, \tilde{\tau}}^{2},
$$

for $u \in \mathscr{S}\left(\overline{\mathbb{R}_{+}^{N}}\right)$ and $\tau \geq \tau_{0}$.

4.2. Local setting and statement of the Carleman estimate. To explain the construction of the phase function, it is useful to use a particular set of coordinates. We set $Z=\left(0, S_{0}\right) \times \Omega$ and $\partial Z=\left(0, S_{0}\right) \times \partial \Omega$.

Let $z_{0}=\left(s_{0}, x_{0}\right) \in \partial Z$. In a neighborhood $V$ of $z_{0}$ in $\mathbb{R}^{N}$, using normal geodesic coordinates for the $x$ variable, we can express the principal part of the Laplace operator $A$ in the following form

$$
A=D_{x_{d}}^{2}+R\left(x, D_{x^{\prime}}\right)
$$

where $R\left(x, D_{x^{\prime}}\right)$ is a tangential differential operator of order 2 with principal symbol $r\left(x, \xi^{\prime}\right)$,

$$
r\left(x, \xi^{\prime}\right) \geq C\left|\xi^{\prime}\right|^{2},
$$

where $C>0$. We denote by $\tilde{r}\left(x, \xi^{\prime}, \eta^{\prime}\right)$ the associated real symmetric bilinear form. The boundary $\left(0, S_{0}\right) \times \partial \Omega$ is locally given by $\left\{z_{N}=0\right\}=\left\{x_{d}=0\right\}$.

Without any loss of generality we shall assume that $V$ is a bounded open set.

We then let $\psi(z)$ be defined in $\mathbb{R}^{N}$ and fulfilling the properties listed in (4.2) with moreover,

$$
\partial_{x_{d}} \psi(z)=\partial_{z_{N}} \psi(z) \geq C>0, \quad z \in V,
$$

and we set $\varphi_{\gamma, \varepsilon}(z)=\exp \left(\gamma \psi_{\varepsilon}(z)\right)$ with $\psi_{\varepsilon}(z)=\psi\left(\varepsilon s, \varepsilon x^{\prime}, x_{d}\right)$, for $\gamma \geq 1$ and $\varepsilon \in[0,1]$. As mentioned above, we shall often write $\varphi$ in place if $\varphi_{\gamma, \varepsilon}$ for the sake of concision.

The main result of this section is the following Carleman estimate.

Theorem 4.17. Let $P=D_{s}^{4}+A^{2}$. Let $z_{0}=\left(s_{0}, x_{0}\right) \in\left(0, S_{0}\right) \times \partial \Omega$. Let $\varphi(z)=\varphi_{\gamma, \varepsilon}(z)$ be defined as above. There exists an open neighborhood $W$ of $z_{0}$ in $\left(0, S_{0}\right) \times \mathbb{R}^{d}, W \subset V$, and there exist $\tau_{0} \geq \tau_{*}$, $\gamma_{0} \geq 1, \varepsilon_{0} \in(0,1]$, and $C>0$ such that

$$
\begin{aligned}
\gamma \sum_{|\alpha| \leq 4}\left\|\tilde{\tau}^{3-|\alpha|} e^{\tau \varphi} D_{s, x}^{\alpha} u\right\|_{+}+\sum_{0 \leq j \leq 3}\left|e^{\tau \varphi} D_{x_{d}}^{r} u_{\mid \partial Z}\right|_{7 / 2-j \tilde{\tau}} & \\
& \leq C\left(\left\|e^{\tau \varphi} P u\right\|_{+}+\sum_{j=0,1} \mid e^{\tau \varphi} D_{x_{d}}^{j} u_{|\partial Z|_{7 / 2-j, \tilde{\tau}}}\right),
\end{aligned}
$$

for $\tau \geq \tau_{0}, \gamma \geq \gamma_{0}, \varepsilon \in\left[0, \varepsilon_{0}\right]$, and for $u=w_{\mid Z}$, with $w \in \mathscr{C}_{c}^{\infty}\left(\left(0, S_{0}\right) \times \mathbb{R}^{d}\right)$ and $\operatorname{supp}(w) \subset W$.

As written in Case (iii) of Section 1.3, the proof we provide of this theorem is based on a decomposition of phase-space in three microlocal regions and the derivation of an adapted estimate in each one of these regions. The definition of these three regions is based on the properties of the roots of the principal symbol of $P$ viewed as a polynomial function of degree four in the variable $\xi_{d}$. We start with the analysis of those properties in the next section and define the microlocal regions in Section 4.4 below. In section 4.5 we provide a proof scheme for a microlocal Carleman estimate in each of the three regions. Then, in Sections 4.6-4.8 we precisely state and prove the microlocal estimate associated with each region. Finally, in Section 4.9 the various microlocal estimates are patched together, to yield the estimate of Theorem 4.17. 
4.3. Root properties. Here, $z$ will be assumed to be an element of $V$ so that all the symbols are well defined. We write, as in Sections 2 and 3,

$$
P=P_{2} P_{1}, \quad \text { with } P_{k}=(-1)^{k} i D_{s}^{2}+A .
$$

Setting $P_{\varphi}=e^{\tau \varphi} P e^{-\tau \varphi}$ we have

$$
P_{\varphi}=Q_{2} Q_{1}, \quad \text { with } Q_{k}=e^{\tau \varphi} P_{k} e^{-\tau \varphi}=(-1)^{k} i\left(D_{s}+i \tau \partial_{s} \varphi(z)\right)^{2}+A_{\varphi},
$$

with, in the selected normal geodesic coordinates,

$$
A_{\varphi}=e^{\tau \varphi} A e^{-\tau \varphi}=\left(D_{x_{d}}+i \tau \partial_{x_{d}} \varphi(z)\right)^{2}+R\left(x, D_{x^{\prime}}+i \tau d_{x^{\prime}} \varphi(z)\right), \quad z=(s, x) .
$$

In fact, we shall write $Q_{k}$ in the following form

$$
Q_{k}=\left(D_{x_{d}}+i \tau \partial_{x_{d}} \varphi(z)\right)^{2}+M_{k}, \quad M_{k}=(-1)^{k} i\left(D_{s}+i \tau \partial_{s} \varphi(z)\right)^{2}+R\left(x, D_{x^{\prime}}+i \tau d_{x^{\prime}} \varphi(z)\right) .
$$

This form will allow us, when a smooth square root $H_{k}$ of $M_{k}$ is available in the tangential calculus associated with $g_{\mathrm{T}}$, to write, up to lower order terms,

$$
Q_{k}=\left(D_{x_{d}}+i \tau \partial_{x_{d}} \varphi+i H_{k}\right)\left(D_{x_{d}}+i \tau \partial_{x_{d}} \varphi-i H_{k}\right),
$$

and, then, we shall base our derivation of a Carleman estimate for $P$ on estimates for first-order factors. This approach was introduced in the seminal work of A.-P. Calderón [Ca158]. It has been used recently to address boundary and interface problems in the derivation of Carleman estimates [LL13, CR14]. Of course, the two smooth square roots, $H_{1}$ and $H_{2}$, may not always be available. Still, on the occurrence of such a case, we shall find that the operators $Q_{1}$ and $Q_{2}$ will be characterized by perfectly elliptic estimates at the boundary, that is, one can estimate the semi-classical Sobolev norm of the solution in $\Omega$ as well as the counterpart norms for the traces of normal derivatives of the solution on $\partial \Omega$ (with the natural $1 / 2$ derivative shift for the traces) -see Section B.1. As a preliminary to this analysis, we shall study the properties of the principal symbols of $Q_{1}$ and $Q_{2}$ and the properties of their roots.

We denote the principal parts of $Q_{k}$ and $M_{k}$ by $q_{k}$ and $m_{k}$, which gives, with $\varrho=(z, \zeta, \tau, \gamma, \varepsilon)$ and $\zeta=(\sigma, \xi)$,

$$
q_{k}(\varrho)=\left(\xi_{d}+i \tau \partial_{x_{d}} \varphi(z)\right)^{2}+m_{k}\left(\varrho^{\prime}\right)=\left(\xi_{d}+i \hat{\tau}_{\xi_{d}}\left(\varrho^{\prime}\right)\right)^{2}+m_{k}\left(\varrho^{\prime}\right)
$$

with

$$
m_{k}\left(\varrho^{\prime}\right)=(-1)^{k} i\left(\sigma+i \hat{\tau}_{\sigma}\left(\varrho^{\prime}\right)\right)^{2}+r\left(x, \xi^{\prime}+i \hat{\tau}_{\xi^{\prime}}\left(\varrho^{\prime}\right)\right),
$$

recalling the definition of $\hat{\tau}\left(\varrho^{\prime}\right)$ introduced in Section 4.1.4 and using the notation (4.7)-(4.8).

For $\hat{t}=\left(\hat{t}_{\sigma}, \hat{t}_{\xi}\right) \in \mathbb{R} \times \mathbb{R}^{d}$, with $\hat{t}_{\xi}=\left(\hat{t}_{\xi^{\prime}}, \hat{t}_{\xi_{d}}\right) \in \mathbb{R}^{d-1} \times \mathbb{R}$, we set

$$
\hat{q}_{k}(z, \zeta, \hat{t})=\left(\xi_{d}+i \hat{t}_{\xi_{d}}\right)^{2}+\hat{m}_{k}\left(z, \zeta^{\prime}, \hat{t}\right), \quad \hat{m}_{k}\left(z, \zeta^{\prime}, \hat{t}\right):=(-1)^{k} i\left(\sigma+i \hat{t}_{\sigma}\right)^{2}+r\left(x, \xi^{\prime}+i \hat{t}_{\xi^{\prime}}\right) .
$$

We have $q_{k}(\varrho)=\hat{q}_{k}(z, \zeta, \hat{\tau})$ and $m_{k}\left(\varrho^{\prime}\right)=\hat{m}_{k}\left(z, \zeta^{\prime}, \hat{\tau}\right)$.

We now study the roots of $\hat{q}_{k}\left(z, \zeta^{\prime}, \xi_{d}, \hat{t}\right)$, with $\zeta^{\prime}=\left(\sigma, \xi^{\prime}\right)$, when viewed as a polynomial in the variable $\xi_{d}$, with the other variables, $z, \zeta^{\prime}$, and $\hat{t}$ acting as parameters. To that purpose, we introduce the following quantity

$$
\hat{\mu}_{k}\left(z, \zeta^{\prime}, \hat{t}\right):=4 \hat{t}_{\xi_{d}}^{2} \operatorname{Re} \hat{m}_{k}\left(z, \zeta^{\prime}, \hat{t}\right)-4 \hat{t}_{\xi_{d}}^{4}+\left(\operatorname{Im} \hat{m}_{k}\left(z, \zeta^{\prime}, \hat{t}\right)\right)^{2} .
$$

We choose $\hat{h}_{k}\left(z, \zeta^{\prime}, \hat{t}\right) \in \mathbb{C}$ such that

$$
\operatorname{Re} \hat{h}_{k}\left(z, \zeta^{\prime}, \hat{t}\right) \geq 0 \text { and } \hat{h}_{k}\left(z, \zeta^{\prime}, \hat{t}\right)^{2}=\hat{m}_{k}\left(z, \zeta^{\prime}, \hat{t}\right) .
$$

We may then write

$$
\hat{q}_{k}(z, \zeta, \hat{t})=\left(\xi_{d}+i \hat{t}_{\xi_{d}}\right)^{2}+\hat{h}_{k}^{2}\left(z, \zeta^{\prime}, \hat{t}\right)=\left(\xi_{d}-\hat{\rho}_{k,+}\left(z, \zeta^{\prime}, \hat{t}\right)\right)\left(\xi_{d}-\hat{\rho}_{k,-}\left(z, \zeta^{\prime}, \hat{t}\right)\right),
$$


with

$$
\hat{\rho}_{k, \pm}\left(z, \zeta^{\prime}, \hat{t}\right)=-i \hat{t}_{\xi_{d}} \pm i \hat{h}_{k}\left(z, \zeta^{\prime}, \hat{t}\right) .
$$

The choice of $\hat{h}_{k}$ is unique if $\hat{m}_{k} \notin \mathbb{R}_{-}$. The results of this section are yet valid in the case $\hat{m}_{k} \in \mathbb{R}_{-}$; however, in the following sections, those results based on the factorization (4.29) will only be used in settings where $\hat{m}_{k} \in \mathbb{R}_{-}$does not occur.

We give some properties of the roots $\hat{\rho}_{k, \pm}\left(z, \zeta^{\prime}, \hat{t}\right)$.

Lemma 4.18. We assume that $\hat{t}_{\xi_{d}} \geq 0$. Let $k=1$ or 2 . The roots $\hat{\rho}_{k,+}\left(z, \zeta^{\prime}, \hat{t}\right)$ and $\hat{\rho}_{k,-}\left(z, \zeta^{\prime}, \hat{t}\right)$ are both homogeneous of degree one in $\left(\zeta^{\prime}, \hat{t}\right)$, and such that

$$
\operatorname{Im} \hat{\rho}_{k,-} \leq-\hat{t}_{\xi_{d}} \leq \operatorname{Im} \hat{\rho}_{k,+} .
$$

We also have

$$
\hat{\rho}_{k,-}=\hat{\rho}_{k,+} \quad \Leftrightarrow \quad \hat{\rho}_{k,-}=\hat{\rho}_{k,+}=-i \hat{t}_{\xi_{d}} \quad \Leftrightarrow \quad \hat{m}_{k}=0 .
$$

Moreover, if $\hat{t}_{\xi_{d}}>0$, we have

$$
\operatorname{Im} \hat{\rho}_{k,+} \lesseqgtr 0 \quad \Leftrightarrow \quad \hat{\mu}_{k} \lesseqgtr 0 .
$$

In particular, if $\hat{t}_{\xi_{d}}>0$, observe that the root $\hat{\rho}_{k,-}$ remains in the lower half complex plane, independently of the values of $z, \zeta^{\prime}$, and $\hat{t}$, while the root $\hat{\rho}_{k,+}$ may cross the real line.

Proof. The roots can be chosen continuous with respect to $\zeta$ and $\hat{t}$ and homogeneity comes naturally. Observe that $\operatorname{Im} \hat{\rho}_{k, \pm}=-\hat{t}_{\xi_{d}} \pm \operatorname{Re} \hat{h}_{k}$. As $\operatorname{Re} \hat{h}_{k} \geq 0$ then (4.31) is clear. The form of $\hat{\rho}_{k, \pm}$ above yields the equivalences in (4.32).

Finally, as $\operatorname{Im} \hat{\rho}_{k,+} \lesseqgtr 0$ is equivalent to $\operatorname{Re} \hat{h}_{k} \lesseqgtr \hat{t}_{\xi_{d}}$, Lemma 4.19 below implies (4.33), since $\operatorname{Re} \hat{h}_{k} \geq 0$ and $\hat{t}_{\xi_{d}}>0$.

Lemma 4.19. Let $t \in \mathbb{C}$ and $m=t^{2}$. We then have, for $x_{0} \in \mathbb{R}$ such that $x_{0} \neq 0$,

$$
|\operatorname{Re} t| \lesseqgtr\left|x_{0}\right| \quad \Leftrightarrow \quad 4 x_{0}^{2} \operatorname{Re} m-4 x_{0}^{4}+(\operatorname{Im} m)^{2} \lesseqgtr 0 .
$$

Proof. Let $t=x+i y$. We have $\operatorname{Re} m=x^{2}-y^{2}$ and $\operatorname{Im} m=2 x y$ and we observe that

$$
4 x_{0}^{2} \operatorname{Re} m-4 x_{0}^{4}+(\operatorname{Im} m)^{2}=4\left(x_{0}^{2}+y^{2}\right)\left(x^{2}-x_{0}^{2}\right),
$$

which gives the result.

Corollary 4.20. We assume that $\hat{t}_{\xi_{d}}>0$. Let $k=1$ or 2 . If $C>0$, there exists $C^{\prime}>0$ such that

$$
\hat{\mu}_{k}\left(z, \zeta^{\prime}, \hat{t}\right) \geq C\left(|\hat{t}|^{2}+\left|\zeta^{\prime}\right|^{2}\right)^{2} \Rightarrow \operatorname{Im} \hat{\rho}_{k,+}\left(z, \zeta^{\prime}, \hat{t}\right) \geq C^{\prime} \hat{\lambda}_{\mathrm{T}}, \quad \hat{\lambda}_{\mathrm{T}}=\left(|\hat{t}|^{2}+\left|\zeta^{\prime}\right|^{2}\right)^{1 / 2},
$$

for $\left(z, \zeta^{\prime}, \hat{t}\right) \in \overline{V \cap \mathbb{R}_{+}^{N}} \times \mathbb{R}^{N-1} \times \mathbb{R}^{N}$.

Proof. We consider the compact set (recall that $V$ is bounded)

$$
\mathscr{C}=\left\{\left(z, \zeta^{\prime}, \hat{t}\right) \in \overline{V \cap \mathbb{R}_{+}^{N}} \times \mathbb{R}^{N-1} \times \mathbb{R}^{N} ; \hat{\lambda}_{\mathrm{T}}=1\right\} .
$$

The inequality $\hat{\mu}_{k} \geq C$ yields a compact set $K$ of $\mathscr{C}$. By (4.33) in Lemma 4.18, we have $\operatorname{Im} \hat{\rho}_{k,+} \geq$ $C^{\prime}>0$ on $K$, and we conclude by homogeneity.

Proposition 4.21. We assume that $\hat{t}_{\xi_{d}} \geq 0$. Let $k=1$ or 2 , we have the following properties:

(1) There exist $\theta_{0} \in(0,1)$ and $C>0$ such that if

$$
z \in V \text { and }|\hat{t}| \leq \theta_{0} \hat{\lambda}_{\mathrm{T}},
$$

then the roots $\hat{\rho}_{k, \pm}$ are simple and non real, and moreover

$$
\operatorname{Im} \hat{\rho}_{k,+} \geq C \hat{\lambda}_{\mathrm{T}}, \quad \operatorname{Im} \hat{\rho}_{k,-} \leq-C \hat{\lambda}_{\mathrm{T}} \quad\left(z, \zeta^{\prime}, \hat{t}\right) \in V \times \mathbb{R}^{N-1} \times \mathbb{R}^{N},
$$

with $\hat{\lambda}_{\mathrm{T}}=\left(|\hat{t}|^{2}+\left|\zeta^{\prime}\right|^{2}\right)^{1 / 2}$. 
(2) There exists $C>0$ such that

$$
0 \leq \hat{t}_{\xi_{d}} \leq C\left(\left|\hat{t}^{\prime}\right|+\left|\zeta^{\prime}\right|\right), \quad \text { and }\left|\zeta^{\prime}\right| \leq C|\hat{t}|,
$$

if $\hat{\rho}_{k,+} \in \mathbb{R}$, where $\hat{t}^{\prime}=\left(\hat{t}_{\sigma}, \hat{t}_{\xi^{\prime}}\right)$. In such case, the value of the imaginary part of the second root is prescribed and nonpositive: $\operatorname{Im} \hat{\rho}_{k,-}=-2 \hat{t}_{\xi_{d}}$.

(3) There exists $C>0$ such that $\left|\hat{t}^{\prime}\right| / C \leq\left|\zeta^{\prime}\right| \leq C\left|\hat{t}^{\prime}\right|$, if $\hat{q}_{k}$ has a double root.

Finally, if $\hat{t}_{\xi_{d}}>0$ and if $\left|\hat{t}^{\prime}\right| / \hat{t}_{\xi_{d}}$ is sufficiently small, and if the polynomial $\hat{q}_{k}, k=1$ or 2 , has a double root, then both roots of the second symbol, $\hat{q}_{k^{\prime}}$ with $k^{\prime} \neq k$, are in the lower half complex plane. More precisely, there exist $C_{0}, C_{1}>0$ such that if $\left|\hat{t}^{\prime}\right| \leq C_{0} \hat{t}_{\xi_{d}}$ then

$$
\hat{\rho}_{k,+}=\hat{\rho}_{k,-} \Rightarrow \operatorname{Im} \hat{\rho}_{k^{\prime}, \pm} \leq-C_{1} \hat{t}_{\xi_{d}} .
$$

Proof. Proof of point (1). Because of homogeneity it is sufficient to assume that $\left(\zeta^{\prime}, \hat{t}\right)$ is on the sphere $\mathbb{S}=\left\{\hat{\lambda}_{\mathrm{T}}=1\right\}$. If $\hat{t}=0$, then we have $\hat{m}_{k}=\hat{h}_{k}^{2}=r\left(x, \xi^{\prime}\right)+(-1)^{k} i \sigma^{2}$. Observe that $\hat{m}_{k} \neq 0$ here. Otherwise $\sigma=0$ and $\xi^{\prime}=0$, which cannot hold as $\left|\zeta^{\prime}\right|=1$. Moreover $\operatorname{Re} \hat{m}_{k} \geq 0$. Hence, we have $\operatorname{Re} \hat{h}_{k}>0$. Then we write

$$
\hat{q}_{k}=\xi_{d}^{2}+\hat{h}_{k}^{2}=\left(\xi_{d}+i \hat{h}_{k}\right)\left(\xi_{d}-i \hat{h}_{k}\right),
$$

yielding $\hat{\rho}_{k,-}=-i \hat{h}_{k}$ and $\hat{\rho}_{k,+}=i \hat{h}_{k}$, which gives $\operatorname{Im} \hat{\rho}_{k,-}<0$ and $\operatorname{Im} \hat{\rho}_{k,+}>0$. As $\mathbb{S} \cap\{\hat{t}=0\}$ is compact we find that $\operatorname{Im} \hat{\rho}_{k,-} \leq-C<0$ and $\operatorname{Im} \hat{\rho}_{k,+} \geq C>0$, for some $C>0$, for $\left|\zeta^{\prime}\right|=1$ and $\hat{t}=0$. Then, using a compactness argument once more, using the continuity of the roots, there exist $\theta_{0} \in(0,1)$ such that

$$
\operatorname{Im} \hat{\rho}_{k,-}\left(z, \zeta^{\prime}, \hat{t}\right) \leq-C^{\prime}<0, \quad \operatorname{Im} \hat{\rho}_{k,+}\left(z, \zeta^{\prime}, \hat{t}\right) \geq C^{\prime}>0,
$$

if $z \in \bar{V}$ and $|\hat{t}| \leq \theta_{0}$, recalling that $V$ is bounded. We then obtain (4.34) in $V$ by homogeneity. In particular this excludes having double roots and real roots.

Proof of point (2). Observe that the inequality $\left|\zeta^{\prime}\right| \leq C \mid \hat{t}$, in the case of a real root is simply another formulation of part of point (1). Next, we observe that $\left|\hat{m}_{k}\right| \lesssim\left|\hat{t}^{\prime}\right|^{2}+\left|\zeta^{\prime}\right|^{2}$ implies $\left|\operatorname{Re} \hat{h}_{k}\right| \lesssim\left|\hat{t}^{\prime}\right|+\left|\zeta^{\prime}\right|$. Since having $\hat{\rho}_{k,+} \in \mathbb{R}$ is equivalent to $\operatorname{Re} \hat{h}_{k}=\hat{t}_{\xi_{d}}$ by (4.30), we thus obtain $\hat{t}_{\xi_{d}} \lesssim\left|\hat{t}^{\prime}\right|+\left|\zeta^{\prime}\right|$. As $\operatorname{Im} \hat{\rho}_{k,-}=-\hat{t}_{\xi_{d}}-\operatorname{Re} \hat{h}_{k}$, we then have $\operatorname{Im} \hat{\rho}_{k,-}=-2 \hat{t}_{\xi_{d}}$.

Proof of point (3) The equation $\hat{m}_{k}=0$, which is equivalent to having a double root, reads

$$
r\left(x, \xi^{\prime}\right)-r\left(x, \hat{t}_{\xi^{\prime}}\right)-(-1)^{k} 2 \sigma \hat{t}_{\sigma}=0, \quad \sigma^{2}-\hat{t}_{\sigma}^{2}+(-1)^{k} 2 \tilde{r}\left(x, \xi^{\prime}, \hat{t}_{\xi^{\prime}}\right)=0,
$$

with $\tilde{r}\left(x, \xi^{\prime}, \eta^{\prime}\right)$ defined below (4.19). From (4.36), using that $r(x,$.$) is uniformly positive definite,$ we obtain

$$
\left|\xi^{\prime}\right|^{2} \lesssim\left|\hat{\xi}_{\xi^{\prime}}\right|^{2}+|\sigma|\left|\hat{t}_{\sigma}\right|, \quad|\sigma|^{2} \lesssim\left|\hat{t}_{\sigma}\right|^{2}+\left|\xi^{\prime}\right|\left|\hat{\xi}_{\xi^{\prime}}\right|
$$

The sum of the two estimates gives $\left|\zeta^{\prime}\right|^{2} \lesssim\left|\hat{t}^{\prime}\right|^{2}+|\sigma \|| \hat{t}_{\sigma}|+| \xi^{\prime}|| \hat{t}_{\xi^{\prime}} \mid$, and with the Young inequality we obtain $\left|\zeta^{\prime}\right| \lesssim\left|\hat{t}^{\prime}\right|$. Similarly, from (4.36) we obtain

$$
\left|\hat{t}_{\xi^{\prime}}\right|^{2} \lesssim\left|\xi^{\prime}\right|^{2}+\left.|\sigma \|| \hat{t}_{\sigma}|, \quad| \hat{t}_{\sigma}\right|^{2} \lesssim|\sigma|^{2}+\left|\xi^{\prime}\right|\left|\hat{t}_{\xi^{\prime}}\right|,
$$

and the sum of the two estimates gives $\left|\hat{t}^{\prime}\right|^{2} \lesssim\left|\zeta^{\prime}\right|^{2}+|\sigma|\left|\hat{t}_{\sigma}\right|+\left|\xi^{\prime}\right|\left|\hat{\xi}_{\xi^{\prime}}\right|$, and with the Young inequality we obtain $\left|\hat{t}^{\prime}\right| \lesssim\left|\zeta^{\prime}\right|$.

Note that we could deduce that $\left|\zeta^{\prime}\right| \lesssim|\hat{t}|$ from point (1). Here, we have obtained a sharper estimate.

Proof of (4.35). If $\hat{q}_{k}$ has a double root, then $\left|\hat{t}^{\prime}\right| \asymp\left|\zeta^{\prime}\right|$ by point (3). Let $\delta \in(0,1)$, and set $C_{1}=1-\delta$. To have $\operatorname{Im} \hat{\rho}_{k^{\prime}, \pm} \leq-C_{1} \hat{t}_{\xi_{d}}$ it suffices to have $\operatorname{Im} \hat{\rho}_{k^{\prime},+} \leq-C_{1} \hat{t}_{\xi_{d}}$ by Lemma 4.18. With 
the notation of the proof of that lemma, this reads $-\hat{t}_{\xi_{d}}+\operatorname{Re} \hat{h}_{k^{\prime}} \leq-C_{1} \hat{t}_{\xi_{d}}$, that is $0 \leq \operatorname{Re} \hat{h}_{k^{\prime}} \leq \delta \hat{t}_{\xi_{d}}$. Now as we have $\left|\operatorname{Re} \hat{h}_{k^{\prime}}\right| \leq\left|\hat{h}_{k^{\prime}}\right| \leq\left|\hat{m}_{k^{\prime}}\right|^{1 / 2} \lesssim\left|\hat{t}^{\prime}\right|+\left|\zeta^{\prime}\right|$, we find that $0 \leq \operatorname{Re} \hat{h}_{k^{\prime}} \lesssim\left|\hat{t}^{\prime}\right|$ here. The result thus follows if we assume that $\left|\hat{t}^{\prime}\right| / \hat{t}_{\xi_{d}}$ is chosen sufficiently small.

Lemma 4.22. Assume that $\left|\hat{t}^{\prime}\right| \leq C_{0} \hat{t}_{\xi_{d}}$ for some $C_{0}>0$. There exists $\delta_{0}>0$ such that if $\delta \in\left(0, \delta_{0}\right)$ and $\hat{\mu}_{k}\left(z, \zeta^{\prime}, \hat{t}\right) \geq-\delta \hat{\lambda}_{\mathrm{T}}^{4}$, with $\hat{\lambda}_{\mathrm{T}}^{2}=|\hat{t}|^{2}+\left|\zeta^{\prime}\right|^{2}$, then the roots of $\hat{q}_{k}$ are simple.

Proof. Because of homogeneity it is sufficient to work on the sphere $\mathbb{S}=\left\{\hat{\lambda}_{T}=1\right\}$. Writing $\hat{m}_{k}=\hat{h}_{k}^{2}$ with $\operatorname{Re} \hat{h}_{k} \geq 0$ as in (4.28), we observe that $\hat{\mu}_{k} \geq-\delta$ reads

$$
4\left(\hat{t}_{\xi_{d}}^{2}+\left(\operatorname{Im} \hat{h}_{k}\right)^{2}\right)\left(\left(\operatorname{Re} \hat{h}_{k}\right)^{2}-\hat{t}_{\xi_{d}}^{2}\right) \geq-\delta,
$$

using the computation of the proof of Lemma 4.19 with $x_{0}=\hat{t}_{\xi_{d}}$. Assume that we have a double root. In such case $\hat{m}_{k}=0$ by Lemma 4.18 and $\left|\hat{t}^{\prime}\right| \asymp\left|\zeta^{\prime}\right|$ by point (3) of Proposition 4.21. We then have $\hat{h}_{k}=0$, yielding $4 \hat{t}_{\xi_{d}}^{4} \leq \delta=\delta \hat{\lambda}_{\mathrm{T}}^{4} \lesssim \delta \hat{t}_{\xi_{d}}^{4}$, using that $\left|\hat{t}^{\prime}\right| \leq C_{0} \hat{\xi}_{\xi_{d}}$. Thus, for $\delta$ chosen sufficiently small we reach a contradiction.

Lemma 4.23. Let $k=1$ or 2 . If both $\delta>0$ and $\left|\hat{t}^{\prime}\right| / \hat{t}_{\xi_{d}}$ are sufficiently small, there exists $C>0$ such that for $\left(z, \zeta^{\prime}, \hat{t}\right) \in \overline{V \cap \mathbb{R}_{+}^{N}} \times \mathbb{R}^{N-1} \times \mathbb{R}^{N}$

$$
\hat{\mu}_{k}\left(z, \zeta^{\prime}, \hat{t}\right) \geq-\delta \hat{\lambda}_{\mathrm{T}}^{4} \Rightarrow|\hat{t}| \leq C\left|\zeta^{\prime}\right|,
$$

with $\hat{\lambda}_{\mathrm{T}}^{2}=\left.\left|\hat{t}^{2}+\right| \zeta^{\prime}\right|^{2}$.

Proof. Because of homogeneity it is sufficient to work on the sphere $\mathbb{S}=\left\{\hat{\lambda}_{\mathrm{T}}=1\right\}$.

Let us now assume that the implication does not hold. Then there exists $\left(z^{(n)}, \zeta^{(n)}, \hat{t}^{(n)}\right) \in$ $\overline{V \cap \mathbb{R}_{+}^{N}} \times \mathbb{S}$, such that $\hat{\mu}_{k}\left(z^{(n)}, \zeta^{\prime(n)}, \hat{t}^{(n)}\right) \geq-\delta$ and $\left|\hat{t}^{(n)}\right|>n\left|\zeta^{(n)}\right|$. As $\left(z^{(n)}, \zeta^{(n)}, \hat{t}^{(n)}\right)$ lays in a compact set (recall that $V$ is bounded), it converges, up to a subsequence, to $\left(z^{(\infty)}, \zeta^{(\infty)}, \hat{t}^{(\infty)}\right) \in \overline{V \cap \mathbb{R}_{+}^{N}} \times \mathbb{S}$. We find that $\zeta^{\prime(\infty)}=0$ and $\hat{m}_{k}\left(z^{(\infty)}, 0, \hat{t}^{(\infty)}\right)=(-1)^{k-1} i\left(\hat{t}_{\sigma}^{(\infty)}\right)^{2}-r\left(x, \hat{t}_{\xi^{\prime}}^{(\infty)}\right)$, yielding

$$
\hat{\mu}_{k}\left(z^{(\infty)}, 0, \hat{t}^{(\infty)}\right)=-4\left(\hat{t}_{\xi_{d}}^{(\infty)}\right)^{2} r\left(x, \hat{t}_{\xi^{\prime}}^{(\infty)}\right)-4\left(\hat{t}_{\xi_{d}}^{(\infty)}\right)^{4}+\left(\hat{t}_{\sigma}^{(\infty)}\right)^{4} \leq-3,
$$

for $\left|\hat{t}^{(\infty) \prime}\right| / \hat{t}_{\xi_{d}}^{(\infty)}$ sufficiently small, as we have $\left|\hat{t}^{(\infty)}\right|=1$. For $\delta$ sufficiently small, we hence reach a contradiction.

4.4. Microlocal regions. With the functions $\hat{\mu}_{k}, k=1,2$, introduced in (4.27) we shall define several microlocal regions. Observe first that $\hat{\mu}_{k}$ is an homogeneous polynomial function of degree four in $\left(\zeta^{\prime}, \hat{t}\right)$. We thus have $\hat{\mu}_{k} \in S_{\mathrm{T}, \hat{t}}^{4}$ in the sense given by Definition 4.1. From Lemma 4.8, we find that we have $\hat{\mu}_{k}\left(z, \zeta^{\prime}, \hat{\tau}\left(\varrho^{\prime}\right)\right) \in S\left(\lambda_{\mathrm{T}, \tilde{\tau}}^{4}, g_{\mathrm{T}}\right)$. We thus define

$$
\mu_{k}\left(\varrho^{\prime}\right):=\lambda_{\mathrm{T}, \tilde{\tau}}^{-4}\left(\varrho^{\prime}\right) \hat{\mu}_{k}\left(z, \zeta^{\prime}, \hat{\tau}\left(\varrho^{\prime}\right)\right) \in S\left(1, g_{\mathrm{T}}\right), \quad k=1,2, \varrho^{\prime}=\left(z, \zeta^{\prime}, \tau, \gamma, \varepsilon\right) .
$$

We recall that $\hat{\tau}=\tau d_{z} \varphi_{\gamma, \varepsilon}(z)=\tilde{\tau}\left(\varrho^{\prime}\right) d_{z} \psi_{\varepsilon}(z)$ and $\psi_{\varepsilon}(z)=\psi\left(\varepsilon z^{\prime}, z_{N}\right)$ with $0<\varepsilon<1$. Observe that we have $\left|\hat{\tau}\left(\varrho^{\prime}\right)\right|=\tilde{\tau}\left(\varrho^{\prime}\right)\left\|d_{z} \psi_{\varepsilon}\right\|_{L^{\infty}} \leq \tilde{\tau}\left(\varrho^{\prime}\right)\left\|d_{z} \psi\right\|_{L^{\infty}}$. Thus, having $0 \leq \tilde{\tau} \leq \delta \theta_{0} \lambda_{\mathrm{T}, \tilde{\tau}}\left(\varrho^{\prime}\right) /\left\|d_{z} \psi\right\|_{L^{\infty}}$, for $\delta \in(0,1]$, implies $\left|\hat{\tau}\left(\varrho^{\prime}\right)\right| \leq \theta_{0} \lambda_{T, \tilde{\tau}}\left(\varrho^{\prime}\right)$. The value $\theta_{0}$ is as introduced in Proposition 4.21. We set $\theta_{1}=\frac{1}{32} \theta_{0} /\left\|d_{z} \psi\right\|_{L^{\infty}}$.

Let $\delta \in(0,1]$ and let $V$ be the bounded open neighborhood in $\mathbb{R}^{N}$ of $z_{0} \in \partial Z$, introduced in Section 4.2. We set $\mathcal{M}_{\mathrm{T}, V}=V \times \mathbb{R}^{N-1} \times\left[\tau_{*},+\infty\right) \times[1,+\infty) \times[0,1]$. We define the following microlocal regions, for $k=1,2$,

$$
\begin{aligned}
& F(V, \delta)=\left\{\varrho^{\prime} \in \mathcal{M}_{\mathrm{T}, V} ; z \in V, \tilde{\tau}\left(\varrho^{\prime}\right) \leq \delta \theta_{1} \lambda_{\mathrm{T}, \tilde{\tau}}\left(\varrho^{\prime}\right)\right\}, \\
& E_{-}^{(k)}(V, \delta)=\left\{\varrho^{\prime} \in \mathcal{M}_{\mathrm{\top}, V} ; z \in V, \mu_{k}\left(\varrho^{\prime}\right) \leq-\delta\right\}, \\
& E_{0}^{(k)}(V, \delta)=\left\{\varrho^{\prime} \in \mathcal{M}_{\mathrm{\top}, V} ; z \in V, \mu_{k}\left(\varrho^{\prime}\right) \geq-\delta\right\} .
\end{aligned}
$$




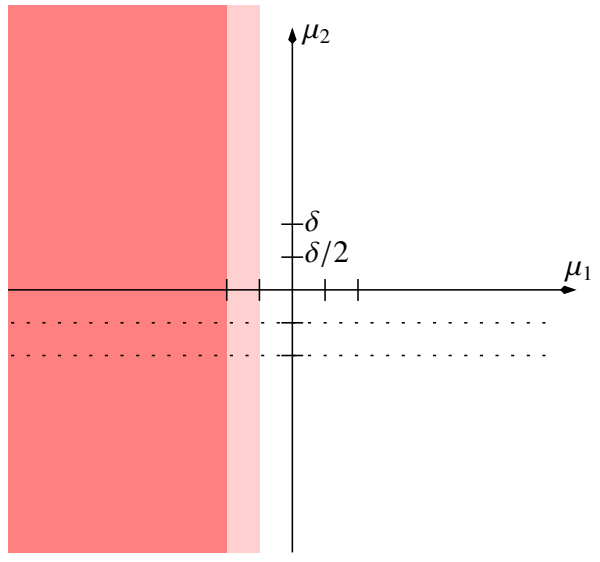

FIGURE 2. Microlocal region $\mathcal{E}_{-}$. In dark color is the region where $\chi_{\delta,-}^{(1)} \equiv$ 1. In light color is the support of $\chi_{\delta,-}^{(1)}$. The boundaries of the associated regions for $\chi_{\delta,-}^{(2)}$ are marked dashed.

Evidently, we have $\mathcal{M}_{\mathrm{T}, V}=E_{-}^{(k)}(V, \delta) \cup E_{0}^{(k)}(V, \delta)$. We now set

$$
\mathcal{E}_{-}(V, \delta)=E_{-}^{(1)}(V, \delta) \cup E_{-}^{(2)}(V, \delta), \quad \mathcal{E}_{0}(V, \delta)=E_{0}^{(1)}(V, \delta) \cap E_{0}^{(2)}(V, \delta),
$$

and we have $\mathcal{M}_{\mathrm{T}, V}=\mathcal{E}_{-}(V, \delta) \cup \mathcal{E}_{0}(V, \delta)$. Below, in the text, when no precision is needed, we shall use the "vague" terminology $F, \mathcal{E}_{-}$, or $\mathcal{E}_{0}$, to refer to microlocal regions that take the forms of $F(V, \delta), \mathcal{E}_{-}(V, \delta), \mathcal{E}_{0}(V, \delta)$.

We let $\chi_{-}, \chi_{0} \in \mathscr{C}^{\infty}(\mathbb{R})$, with values in $[0,1]$, be such that

$$
\begin{aligned}
& \chi_{-} \equiv 1 \text { on }(-\infty,-1], \quad \text { and } \operatorname{supp}\left(\chi_{-}\right) \subset(-\infty,-1 / 2], \\
& \chi_{0} \equiv 1 \text { on }[-2,+\infty),
\end{aligned}
$$

Let $V_{0} \Subset V$ be an open neighborhood of $z_{0}$ in $\mathbb{R}^{N}$ and let $\chi_{V_{0}} \in \mathscr{C}^{\infty}\left(\mathbb{R}^{N}\right)$ be such that $\operatorname{supp}\left(\chi_{V_{0}}\right) \subset V$ and $\chi_{V_{0}} \equiv 1$ in an open neighborhood of $V_{0}$. With $\eta \in \mathscr{C}_{c}^{\infty}\left(-\theta_{1}, \theta_{1}\right)$, with values in $[0,1]$ such that $\eta \equiv 1$ in $\left[-\theta_{1} / 2, \theta_{1} / 2\right]$, we set

$$
\chi_{\delta, F}\left(\varrho^{\prime}\right)=\eta\left(\tilde{\tau}\left(\varrho^{\prime}\right) /\left(\delta \lambda_{\top}, \tilde{\tau}\left(\varrho^{\prime}\right)\right)\right) \in S\left(1, g_{\top}\right) .
$$

and

$$
\chi_{F}\left(\varrho^{\prime}\right)=\chi_{V_{0}}(z) \chi_{1, F}\left(\varrho^{\prime}\right) \in S\left(1, g_{\mathrm{T}}\right)
$$

We set

$$
\chi_{\delta,-}^{(k)}\left(\varrho^{\prime}\right)=\chi_{V_{0}}(z)\left(1-\chi_{1 / 4, F}\left(\varrho^{\prime}\right)\right) \chi_{-}\left(\mu_{k}\left(\varrho^{\prime}\right) / \delta\right) \in S\left(1, g_{\top}\right) .
$$

Observe that we have

$$
\chi_{\delta,-}^{(k)} \equiv 1 \text { on } E_{-}^{(k)}\left(V_{0}, \delta\right) \backslash F(V, 1 / 4), \quad \operatorname{supp}\left(\chi_{\delta,-}^{(k)}\right) \subset E_{-}^{(k)}(V, \delta / 2) \backslash F(V, 1 / 8),
$$

and thus

$$
\chi_{\delta,-}^{(1)}+\chi_{\delta,-}^{(2)} \geq 1 \text { on } \mathcal{E}_{-}\left(V_{0}, \delta\right) \backslash F(V, 1 / 4), \quad \operatorname{supp}\left(\chi_{\delta,-}^{(1)}+\chi_{\delta,-}^{(2)}\right) \subset \mathcal{E}_{-}(V, \delta / 2) \backslash F(V, 1 / 8) .
$$

We finally set

$$
\chi_{\delta, 0}\left(\varrho^{\prime}\right)=\chi_{V_{0}}(z)\left(1-\chi_{1 / 4, F}\left(\varrho^{\prime}\right)\right) \chi_{0}\left(\mu_{1}\left(\varrho^{\prime}\right) / \delta\right) \chi_{0}\left(\mu_{2}\left(\varrho^{\prime}\right) / \delta\right) \in S\left(1, g_{\top}\right) .
$$




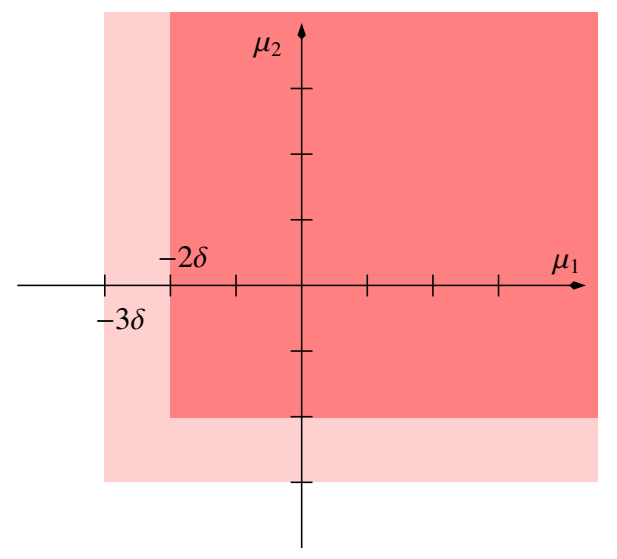

Figure 3. Microlocal region $\mathcal{E}_{0}$. In dark color is the region where $\chi_{\delta, 0} \equiv 1$. In light color is the support of $\chi_{\delta, 0}$.

Observe that we have

$$
\chi_{\delta, 0} \equiv 1 \text { on } \mathcal{E}_{0}\left(V_{0}, 2 \delta\right) \backslash F(V, 1 / 4) \quad \operatorname{supp}\left(\chi_{\delta, 0}\right) \subset \mathcal{E}_{0}(V, 3 \delta) \backslash F(V, 1 / 8),
$$

and

$$
\chi_{F}+\chi_{\delta,-}^{(1)}+\chi_{\delta,-}^{(2)}+\chi_{\delta, 0} \geq 1 \text { on a conic neighborhood of } \mathcal{M}_{\mathrm{T}, V_{0}} .
$$

With the microlocal cutoff functions we have just introduced we associate tangential pseudodifferential operators, all in $\Psi\left(1, g_{\mathrm{T}}\right)$,

$$
\Xi_{F}=\mathrm{Op}_{\mathrm{T}}\left(\chi_{F}\right), \quad \Xi_{\delta,-}^{(k)}=\mathrm{Op}_{\mathrm{T}}\left(\chi_{\delta,-}^{(k)}\right), \quad k=1,2, \quad \text { and } \Xi_{\delta, 0}=\mathrm{Op}_{\mathrm{T}}\left(\chi_{\delta, 0}\right) .
$$

4.5. Proof strategies in the three microlocal regions. Derivations in all three microlocal regions require first the proof of estimates for various factors and second the concatenation of those estimates. For this second part, to avoid redundancies, we describe in Appendix B.4, along with proofs, how various type of estimates can be concatenated.

The estimate associated with region $\mathcal{E}_{-}$is proven in Section 4.6. In region $\mathcal{E}_{-}$, we have $P_{\varphi}=$ $Q_{1} Q_{2}$ where at least one of the factors is characterized by a principal symbol with two roots in the lower half complex plane. This yields for this factor, say $Q_{1}$, a perfectly elliptic estimate at the boundary $\left\{x_{d}=0\right\}$, as given by Lemma B.1 (see Appendix B.1). For the second operator $Q_{2}$, one can derive an estimate whose form is classical and exhibits a loss of a half derivative, as given in Proposition B.10. A proof is provided in Appendix B.5, in particular since the estimate needs to hold uniformly with respect to all parameters introduced. Finally, the two estimates are concatenated to obtain an estimate for $P_{\varphi}$ in $\mathcal{E}_{-}$.

The estimate associated with region $\mathcal{E}_{0}$ is proven in Section 4.7. The treatment this region requires the most delicate argument and justifies the development of the Weyl-Hörmander calculus of Section 4.1. Microlocally, in this region we write $P_{\varphi}=Q_{1} Q_{2}$ and we manage to write each $Q_{k}$, $k=1,2$, in the form

$$
Q_{k}=Q_{k,-} Q_{k,+}+(1+\gamma \varepsilon) R_{1},
$$

where $Q_{k,-}, Q_{k,+}$ and $R_{1}$ are all first-order operators. The operator $Q_{k,-}$ is characterized by a principal symbol with a root in the lower half complex plane. Setting $Q^{-}=Q_{1,-} Q_{2,-}$ and $Q^{+}=Q_{1,+} Q_{2,+}$, with delicate commutator arguments we obtain $P_{\varphi}=Q^{-} Q^{+}+(1+\gamma \varepsilon) R_{3}$ where 
$R_{3} \in \Psi_{\tilde{\tau}}^{2,1}$. We thus manage gather togethers factors with similar root locations without generating a remainder in $\gamma \Psi_{\tilde{\tau}}^{2,1}$. Observe that this latter class for the remainder is obtained if operator commutations within the Weyl-Hörmander calculus are carried invoking usual arguments. Here, to obtain the sharper class $(1+\gamma \varepsilon) \Psi_{\tilde{\tau}}^{2,1}$, we use the precise forms of the involved operators and symbols.

For $Q^{-}$we have a perfectly elliptic estimate at the boundary $\left\{x_{d}=0\right\}$, as given by Lemma B.1 (see Appendix B.1). For each operator $Q_{k,+}$ a sub-elliptic estimate can be obtained with a trace term used as an observation as given by Lemma B.6 in Appendix B.3.3. Concatenated together, two such estimates yield an estimate for $Q_{+}$with a loss of a full derivative and observation terms that involve both the Dirichlet trace and the Neumann trace of the solution. Concatenating now the estimates for $Q^{-}$and $Q^{+}$one obtains microlocally an estimate of the form

$$
\gamma\left\|\tilde{\tau}^{-1} v\right\|_{4,0, \tilde{\tau}}+|\operatorname{tr}(v)|_{3,1 / 2, \tilde{\tau}} \lesssim\left\|Q^{-} Q^{+} v\right\|_{+}+|\operatorname{tr}(v)|_{1,5 / 2, \tilde{\tau}} .
$$

With the form of the remainder term $(1+\gamma \varepsilon) R_{3}$ that appeared above in the decomposition of $P_{\varphi}$ one then sees that a similar estimate can be obtained for $P_{\varphi}$ in place of $Q^{-} Q^{+}$by choosing $\gamma>0$ sufficiently large and $\varepsilon>0$ sufficiently small. Observe that if the remainder term had been in $\gamma \Psi_{\tilde{\tau}}^{2,1}$ we would not have been able to transform the estimate obtained for $Q^{-} Q^{+}$into an estimate for $P_{\varphi}$.

Some technical aspects of the proof in the region $\mathcal{E}_{0}$ described above require to have $\tilde{\tau}\left(\varrho^{\prime}\right)$ of the same order as $\lambda_{\mathrm{T}, \tilde{\tau}}\left(\varrho^{\prime}\right)$. This is however not true in that region. One thus rather considers a region of the form $\mathcal{E}_{0} \backslash F$, since region $F$ is characterized by $\tilde{\tau}\left(\varrho^{\prime}\right) \leq C \lambda_{\mathrm{T}, \tilde{\tau}}\left(\varrho^{\prime}\right)$ for a well chosen constant (see above). A last microlocal region, namely $F$, thus needs to be considered.

The treatment of region $F$ is given in Section 4.8 and has some similarities with what is done in the region $\mathcal{E}_{0} \backslash F$. Yet, the treatment of remainder terms needs not be as refined. The operator $P_{\varphi}$ is written in the form $P_{\varphi}=Q^{-} Q^{+}+\gamma R_{3}$ with $R_{3} \in \Psi_{\tilde{\tau}}^{2,1}$ and again $Q^{-}=Q_{1,-} Q_{2,-}$ and $Q^{+}=Q_{1,+} Q_{2,+}$. Here also, for $Q^{-}$we have a perfectly elliptic estimate at the boundary $\left\{x_{d}=0\right\}$. For $Q^{+}$, the estimate we obtain is very different from what is done in $\mathcal{E}_{0} \backslash F$. The region $F$ is designed so that the roots associated with the factors $Q_{1,+}$ and $Q_{2,+}$ are both located in the upper half complex plane. For each of these operators one can thus obtain a microlocal elliptic estimate at the boundary $\left\{x_{d}=0\right\}$ with one trace used as an observation term yet without any loss of derivative as given in Lemma B.4 in Appendix B.3.1. Put together, with a concatenation argument, an estimate for $Q^{+}$is obtained with observation terms that involve both the Dirichlet trace and the Neumann trace of the solution. This estimate for $Q^{+}$does not exhibit any loss of derivative: it is an elliptic estimate. Concatenated together the estimates for $Q^{+}$and $Q^{-}$yield also an elliptic estimate for $Q^{-} Q^{+}$with the above two traces as observation terms. The elliptic strength of this estimate then allows one to handle the remainder term in $\gamma \Psi_{\tilde{\tau}}^{2,1}$ yielding a similar result for $P_{\varphi}$ in the microlocal region $F$.

As a final step of the proof of Theorem 4.17, we patch together the estimates obtained in the above three microlocal regions. This is done in Section 4.9.

4.6. Microlocal estimate in the region $\mathcal{E}_{-}$. We prove the following estimate.

Proposition 4.24. Let $M \in \mathbb{N}$. Let $k=1$ or 2 . For $\delta \in(0,1)$, there exist $\tau_{0} \geq \tau_{*}, \gamma_{0} \geq 1$, and $C>0$ such that

$$
\gamma^{\frac{1}{2}}\left\|\tilde{\tau}^{-1 / 2} \Xi_{\delta,-}^{(k)} v\right\|_{4,0, \tilde{\tau}}+\left|\operatorname{tr}\left(\Xi_{\delta,-}^{(k)} v\right)\right|_{3,1 / 2, \tilde{\tau}} \leq C\left(\left\|P_{\varphi} \Xi_{\delta,-}^{(k)} v\right\|_{+}+\left|\operatorname{tr}\left(\Xi_{\delta,-}^{(k)} v\right)\right|_{0,7 / 2, \tilde{\tau}}+\|v\|_{4,-M, \tilde{\tau}}\right),
$$

for $\tau \geq \tau_{0}, \gamma \geq \gamma_{0}, \varepsilon \in[0,1]$, and for $v \in \mathscr{S}\left(\overline{\mathbb{R}_{+}^{N}}\right)$.

The term $\|v\|_{4,-M, \tilde{\tau}}$ in the r.h.s. stands as a remainder that will be 'absorbed' once the estimations in the different microlocal regions are patched together. In fact, observe that this term is much 
weaker than that in the 1.h.s. in the Carleman estimate (4.21) of Theorem 4.17. The meaningful observation term in the r.h.s. of (4.40) is $|\operatorname{tr}(v)|_{0,7 / 2, \tilde{\tau}}$, which is of the strength as the terms in the 1.h.s. of (4.21), and can be found in the r.h.s. of that latter estimate.

Proof. We have $P_{\varphi}=Q_{1} Q_{2}$. We consider the case $k=1$. The same proof can be written in the case $k=2$. To ease notation we write $\chi$ in place of $\chi_{\delta,-}$ and $\Xi$ in place of $\Xi_{\delta,-}$.

In a conic neighborhood of $\operatorname{supp}(\chi) \subset \mathcal{M}_{\mathrm{T}, V}$, with $V$ introduced in Section 4.2, we have $\mu_{1} \leq$ $-C \delta$. As (4.20) holds in $V$ we have $\hat{\tau}_{\xi_{d}} \geq C \tilde{\tau}$ and thus $\left|\hat{\tau}_{\xi}\right| \asymp \tilde{\tau}$. By Lemma 4.18, both roots of the symbol $q_{1}$ of the operator $Q_{1}$ are in the lower half complex plane. Thus,

$$
\text { the operator } Q_{1} \text { fulfills the requirements of Lemma B.1. }
$$

Also, for the operator $Q_{2}$, without any assumption on the position of the roots in the complex plane, we have the following estimate, characterized by the loss of a half derivative and a boundary observation term, by Proposition B.10, for $\ell \in \mathbb{R}$,

$$
\gamma^{1 / 2}\left\|\tilde{\tau}^{-1 / 2} \Xi v\right\|_{2, \ell, \tilde{\tau}}+|\operatorname{tr}(\Xi v)|_{1, \ell+1 / 2, \tilde{\tau}} \lesssim\left\|Q_{2} \Xi v\right\|_{0, \ell, \tilde{\tau}}+|\operatorname{tr}(\Xi v)|_{0, \ell+3 / 2, \tilde{\tau}},
$$

for $v \in \mathscr{S}\left(\overline{\mathbb{R}_{+}^{N}}\right)$, for $\tau \geq \tau_{*}$ and $\gamma \geq 1$ chosen sufficiently large, and $\varepsilon \in[0,1]$ (recall that $\operatorname{supp}(\chi) \subset \mathcal{M}_{\top, V}$ which gives $\operatorname{supp}(\Xi v) \subset V^{\prime} \Subset V$, for some open set $V^{\prime}$, thus permitting the application of Proposition B.10).

With (4.41), (4.42), and Proposition B.8, applied with $Q^{-}=Q_{1}$ and $Q^{+}=Q_{2}$ here, and with $\alpha_{1}=0$ and $\alpha_{2}=1$ and $\delta_{1}=1$ and $\delta_{2}=0$, we obtain the result of the proposition, by choosing $\tau \geq \tau_{*}$ and $\gamma \geq 1$ sufficiently large.

4.7. Microlocal estimate in the region $\mathcal{E}_{0} \backslash F$. We prove the following estimate.

Proposition 4.25. Let $M \in \mathbb{N}$. For $\delta_{0} \in(0,1)$ chosen sufficiently small and $\delta \in\left(0, \delta_{0}\right]$, there exist $\tau_{0} \geq \tau_{*}, \gamma_{0} \geq 1, \varepsilon_{0} \in(0,1]$, and $C>0$ such that

$$
\gamma\left\|\tilde{\tau}^{-1} \Xi_{\delta, 0} v\right\|_{4,0, \tilde{\tau}}+\left|\operatorname{tr}\left(\Xi_{\delta, 0} v\right)\right|_{3,1 / 2, \tilde{\tau}} \leq C\left(\left\|P_{\varphi} \Xi_{\delta, 0} v\right\|_{+}+\left|\operatorname{tr}\left(\Xi_{\delta, 0} v\right)\right|_{1,5 / 2, \tilde{\tau}}+\|v\|_{4,-M, \tilde{\tau}}\right),
$$

for $\tau \geq \tau_{0}, \gamma \geq \gamma_{0}, \varepsilon \in\left[0, \varepsilon_{0}\right]$, and for $v \in \mathscr{S}\left(\overline{\mathbb{R}_{+}^{N}}\right)$.

Before giving the proof of this microlocal estimate we need to provide some additional properties of the symbols $m_{k}$ introduced in Section 4.3 and its square root, $h_{k}$. Note that the region $F$ is introduced to isolate the case where $\tilde{\tau} \leq C\left|\zeta^{\prime}\right|$ and this permits to exploit the relation $\left|\zeta^{\prime}\right| \leq C \tilde{\tau}$ in the region $\mathcal{E}_{0} \backslash F$. This is used to obtain some symbol properties of $h_{k}$.

We recall the form of the tangential differential operator $M_{k}$, as introduced in (4.23),

$$
M_{k}:=(-1)^{k} i\left(D_{s}+i \tau \partial_{s} \varphi(z)\right)^{2}+R\left(x, D_{x^{\prime}}+i \tau d_{x^{\prime}} \varphi(z)\right),
$$

whose principal symbol is given by $m_{k}\left(\varrho^{\prime}\right):=(-1)^{k} i\left(\sigma+i \tau \partial_{s} \varphi(z)\right)^{2}+r\left(x, \xi^{\prime}+i \tau d_{x^{\prime}} \varphi(z)\right) \in$ $S\left(\lambda_{\mathrm{T}, \tilde{\tau}}^{2}, g_{\mathrm{T}}\right)$. Observe that we have the following symbol estimation.

Lemma 4.26. We have $\partial_{x_{d}} m_{k} \in S\left((1+\varepsilon \gamma) \lambda_{\mathrm{T}, \tilde{\tau}}^{2}, g_{\mathrm{T}}\right)$.

Proof. We write $m_{k}\left(\varrho^{\prime}\right)=(-1)^{k} i\left(\sigma+i \hat{\tau}_{\sigma}\left(\varrho^{\prime}\right)\right)^{2}+r\left(x, \xi^{\prime}+i \hat{\tau}_{\xi^{\prime}}\left(\varrho^{\prime}\right)\right)$, with the notation of (4.7). We then have

$$
\partial_{x_{d}} m_{k}=-2(-1)^{k}\left(\partial_{x_{d}} \hat{\tau}_{\sigma}\right)\left(\sigma+i \hat{\tau}_{\sigma}\right)+2 i \tilde{r}\left(x, \xi^{\prime}+i \hat{\tau}_{\xi^{\prime}}, \partial_{x_{d}} \hat{\tau}_{\xi^{\prime}}\right)+\partial_{x_{d}} r\left(x, \xi^{\prime}+i \hat{\tau}_{\xi^{\prime}}\right),
$$

with $\tilde{r}\left(x, \xi^{\prime}, \eta^{\prime}\right)$ defined below (4.19). By Lemma 4.5, we have $\hat{\tau}^{\prime}=\left(\hat{\tau}_{\sigma}, \hat{\tau}_{\xi^{\prime}}\right) \in S\left(\varepsilon \tilde{\tau}, g_{T}\right)^{N-1}$ yielding $\partial_{x_{d}} \hat{\tau}^{\prime} \in S\left(\varepsilon \gamma \tilde{\tau}, g_{\mathrm{T}}\right)^{N-1}$, and as $\partial_{x_{d}} r\left(x, \xi^{\prime}+i \hat{\tau}_{\xi^{\prime}}\right) \in S\left(\lambda_{\mathrm{T}, \tilde{\tau}}^{2}, g_{\mathrm{T}}\right)$, the result follows. 
Let $k=1,2$. If $\mu_{k}\left(\varrho^{\prime}\right) \geq-C \delta$, and for $\delta>0$ sufficiently small then $m_{k} \neq 0$ and, equivalently, the roots of $q_{k}(\varrho)$ are simple, by Lemma 4.22 since $\left|\hat{\tau}^{\prime}\right| \lesssim \hat{\tau}_{\xi_{d}}$ for $z \in V$; recall the definition of the operator $Q_{k}, Q_{k}=\left(D_{x_{d}}+i \tau \partial_{x_{d}} \varphi(x)\right)^{2}+M_{k}$, and its principal symbol $q_{k}$ in (4.22)-(4.24).

Lemma 4.27. Let $C, C^{\prime}>0$ and let $\mathscr{U}_{\delta}$ be a conic open set of $\mathcal{M}_{\mathrm{T}, V}$ such that $\mu_{k}\left(\varrho^{\prime}\right) \geq-C \delta$ and $\lambda_{\top, \tilde{\tau}} \leq C^{\prime}|\hat{\tau}(x)|$ in $\mathscr{U}_{\delta}$. For $\delta_{0} \in(0,1)$ and $\varepsilon_{0}>0$ chosen sufficiently small, if $0<\delta \leq \delta_{0}$ and $0 \leq \varepsilon \leq \varepsilon_{0}$, the symbol $m_{k}$ is elliptic and there exists $h_{k} \in S\left(\lambda_{\mathrm{T}, \tilde{\tau}}, g_{\top}\right)$ in $\mathscr{U}_{\delta}$ that is elliptic and that satisfies

$$
h_{k}^{2}=m_{k} \text { and } \operatorname{Re} h_{k} \geq 0 .
$$

Moreover, we have $\partial_{x_{d}} h_{k} \in S\left((1+\varepsilon \gamma) \lambda_{\top, \tilde{\tau}}, g_{\top}\right)$ in $\mathscr{U}_{\delta}$.

The second part of Lemma 4.27 improves, for $h_{k}$, upon the natural behavior of an arbitrary element of $f \in S\left(\lambda_{\mathrm{T}, \tilde{\tau}}, g_{\mathrm{T}}\right)$ for which we have $\partial_{x_{d}} f \in S\left(\gamma \lambda_{\mathrm{T}, \tilde{\tau}}, g_{\mathrm{T}}\right)$. This is a key aspect of our proof strategy of the Carleman estimate. In fact, if one chooses $\varepsilon=0$, that is, a weight function $\psi=\psi\left(x_{d}\right)$, then one finds directly that $\partial_{x_{d}} m_{k} \in S\left(\lambda_{\mathrm{T}, \tilde{\tau}}^{2}, g_{\mathrm{T}}\right)$ and $\partial_{x_{d}} h_{k} \in S\left(\lambda_{\mathrm{T}, \tilde{\tau}}, g_{\mathrm{T}}\right)$, as confirmed by Lemmata 4.26 and 4.27. However, such a weight function is not convex with respect to the boundary $\left\{x_{d}=0\right\}$, which turns out to be an obstruction for the applications of the Carleman estimate we consider here. If we simply let $\psi$ be of the form $\psi\left(s, x^{\prime}, x_{d}\right)$ we then obtain $\partial_{x_{d}} h_{k} \in$ $S\left(\gamma \lambda_{\mathrm{T}, \tilde{\tau}}, g_{\mathrm{T}}\right)$ and the proof scheme for the Carleman estimate collapses: the parameter $\gamma$ needs to be set large, which yields uncontrolled terms in the derivation. The introduction of the parameter $\varepsilon$, writing $\psi_{\varepsilon}(z)=\psi\left(\varepsilon s, \varepsilon x^{\prime}, x_{d}\right)$ is thus designed to control this behavior and to bring the analysis as "close" as we wish to the case $\varepsilon=0$ for the derivation of the estimate and yet preserving some convexity with respect to the boundary $\left\{x_{d}=0\right\}$.

Proof. In $V$, we have $\partial_{x_{d}} \psi \geq C>0$ yielding $|\hat{\tau}| \asymp \hat{\tau}_{\xi_{d}} \asymp \tilde{\tau}$ by Lemma 4.6. Next, $\left|\hat{\tau}^{\prime}\right| / \hat{\tau}_{\xi_{d}}$ can be made as small as needed by choosing $\varepsilon>0$ small. Thus, if we choose $\delta \in\left(0, \delta_{0}\right]$ and $\varepsilon>0$ sufficiently small, by Lemma 4.23 we have $|\hat{\tau}(x)| \lesssim\left|\zeta^{\prime}\right|$ and with the additional assumption made here we obtain

$$
\left|\zeta^{\prime}\right| \asymp \tilde{\tau} \asymp \tilde{\tau}_{\xi_{d}} \quad \text { in } \overline{\mathscr{U}_{\delta}} .
$$

If $m_{k}\left(\varrho^{\prime}\right)$ remains away from a neighborhood of the negative real axis in the complex plane for $\varrho^{\prime} \in \overline{\mathscr{U}_{\delta}}$, we can then define $h_{k}\left(\varrho^{\prime}\right)$ as the principal square root of $m_{k}\left(\varrho^{\prime}\right)$. Then, it is straightforward to obtain $h_{k} \in S\left(\lambda_{\mathrm{T}, \tilde{\tau}}, g_{\mathrm{T}}\right)$ in $\mathscr{U}_{\delta}$. In fact, if we assume $\left|\operatorname{Im} m_{k}\left(\varrho^{\prime}\right)\right| \leq \alpha \lambda_{\mathrm{T}, \tilde{\tau}}^{2}$, as we have, recalling the definition of $\mu_{k}$ in (4.37),

$$
\mu_{k}\left(\varrho^{\prime}\right) \lambda_{\mathrm{T}, \tilde{\tau}}^{4}\left(\varrho^{\prime}\right)=4 \hat{\tau}_{\xi_{d}}^{2} \operatorname{Re} m_{k}\left(\varrho^{\prime}\right)-4 \hat{\tau}_{\xi_{d}}^{4}+\left(\operatorname{Im} m_{k}\left(\varrho^{\prime}\right)\right)^{2}
$$

it yields, using (4.43), $\operatorname{Re} m_{k}\left(\varrho^{\prime}\right) \geq \hat{\tau}_{\xi_{d}}^{2}(1+\mathscr{O}(\delta+\alpha))$. By choosing $\alpha$ and $\delta$ sufficiently small, we obtain $\operatorname{Re} m_{k}\left(\varrho^{\prime}\right) \gtrsim \hat{\tau}_{\xi_{d}}^{2}$ in $\overline{\mathscr{U}_{\delta}}$.

As $m_{k}\left(\varrho^{\prime}\right)$ is homogeneous of degree two, we find that $h_{k}$ is homogeneous of degree one in $\overline{\mathscr{U}_{\delta}}$. Recalling that $z=(x, s)$ remains in a compact domain here, we thus find

$$
\left|h_{k}\left(\varrho^{\prime}\right)\right| \gtrsim \lambda_{\top, \tilde{\tau}} \quad \text { in } \overline{\mathscr{U}_{\delta}} .
$$

Next, as $h_{k}^{2}=m_{k} \neq 0$ in $\overline{\mathscr{U}_{\delta}}$ we may write, with Lemma 4.26 ,

$$
2 h_{k} \partial_{x_{d}} h_{k}=\partial_{x_{d}} m_{k} \in S\left((1+\varepsilon \gamma) \lambda_{\mathrm{T}, \tilde{\tau}}^{2}, g_{\mathrm{T}}\right) .
$$

which yields the result using the ellipticity estimate (4.44). 


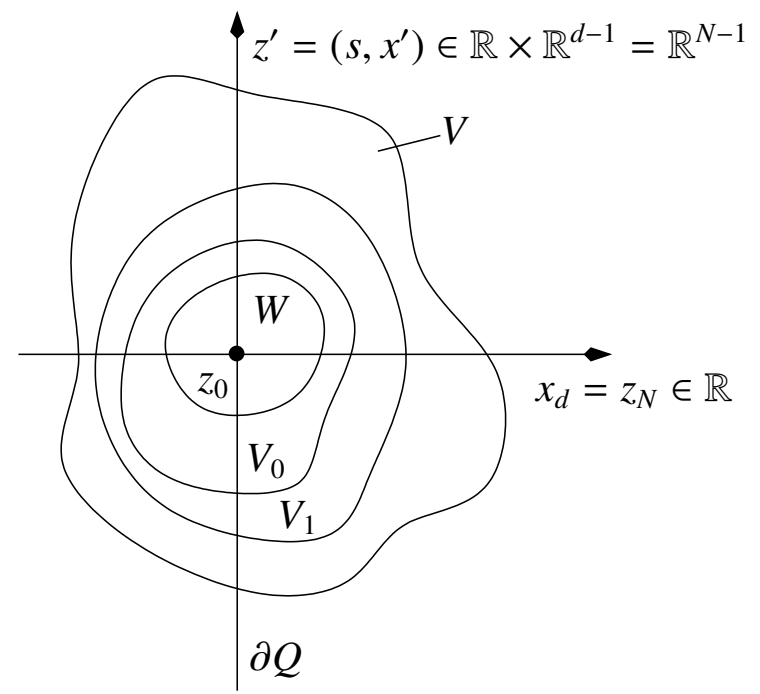

Figure 4. Open neighborhoods of $z_{0} \in \partial Z$ introduced in the course of the proof of Theorem 4.17.

We let $\underline{\chi}_{\delta}, \chi_{\delta, 1} \in S\left(1, g_{\mathrm{T}}\right)$ be supported in $\mathcal{M}_{\mathrm{T}, V}$, homogeneous of degree zero, and be such that $\mu_{k} \geq-C \delta$ for both $k=1,2$ on their supports and $\chi_{\delta, 1} \equiv 1$ in a conic neighborhood of $\operatorname{supp}\left(\chi_{\delta, 0}\right)$ and $\underline{\chi}_{\delta} \equiv 1$ in a conic neighborhood of $\operatorname{supp}\left(\chi_{\delta, 1}\right)$. Recalling the notation of Section 4.4 and the microlocalization symbols constructed there, this can be done as follows, for instance for the construction of $\chi_{\delta, 1}$. Let $\hat{\chi}_{1} \in \mathscr{C}^{\infty}(\mathbb{R})$ be such that

$$
\operatorname{supp}\left(\hat{\chi}_{1}\right) \subset[-4,+\infty), \quad \hat{\chi_{1}} \equiv 1 \text { on a neighborhood of }[-3,+\infty) .
$$

We also introduce $V_{1} \subset V$ an open neighborhood of $\operatorname{supp}\left(\chi_{V_{0}}\right)$ in $\overline{\mathbb{R}_{+}^{N}}$, in particular $V_{0} \Subset V_{1}$ (the local geometry is illustrated in Figure 4) and we choose $\chi_{V_{1}} \in \mathscr{C}^{\infty}\left(\overline{\mathbb{R}_{+}^{N}}\right)$ such that

$$
\chi_{V_{1}} \equiv 1 \text { on a neighborhood of } V_{1}, \quad \operatorname{supp}\left(\chi_{V_{1}}\right) \subset V .
$$

We set

$$
\chi_{\delta, 1}\left(\varrho^{\prime}\right)=\chi_{V_{1}}(z)\left(1-\chi_{1 / 16, F}\left(\varrho^{\prime}\right)\right) \hat{\chi}_{1}\left(\mu_{1}\left(\varrho^{\prime}\right) / \delta\right) \hat{\chi}_{1}\left(\mu_{2}\left(\varrho^{\prime}\right) / \delta\right) \in S\left(1, g_{\top}\right) .
$$

we have $\chi_{\delta, 1} \equiv 1$ in a conic neighborhood of $\operatorname{supp}\left(\chi_{\delta, 0}\right)$.

We choose $\delta_{0}>0$ sufficiently small so that the results of Lemmata 4.22 and 4.23 apply, that is, on $\operatorname{supp}\left(\underline{\chi}_{\delta}\right)$ the roots of $q_{k}$ are simple and $\left|\hat{\tau}\left(\varrho^{\prime}\right)\right| \lesssim\left|\zeta^{\prime}\right|$, and also the result of Lemma 4.27 holds for $\mathscr{U}_{\delta}$ a conic neighborhood of $\operatorname{supp}\left(\underline{\chi}_{\delta}\right)$, for $\delta \in\left(0, \delta_{0}\right)$ and for $\varepsilon>0$ chosen sufficiently small. With the value of $\delta$ fixed now, to ease notation we now write $\underline{\chi}, \chi_{0}, \chi_{1}$ in place of $\underline{\chi}_{\delta}, \chi_{\delta, 0}, \chi_{\delta, 1}$ and $\Xi_{0}, \Xi_{1}$ in place of $\mathrm{Op}_{\mathrm{T}}\left(\chi_{\delta, 0}\right), \mathrm{Op}_{\mathrm{T}}\left(\chi_{\delta, 1}\right)$.

Lemma 4.28. Let $\chi=\chi_{0}$ or $\chi_{1}$ and, accordingly, $\Xi=\Xi_{0}$ or $\Xi_{1}$. We have

$$
\begin{aligned}
Q_{k} \Xi & =Q_{k,+} Q_{k,-} \Xi+(1+\gamma \varepsilon) R_{1} \Xi+R_{-M} \\
& =Q_{k,-} Q_{k,+} \Xi+(1+\gamma \varepsilon) R_{1}^{\prime} \Xi+R_{-M}^{\prime},
\end{aligned}
$$

where $Q_{k, a}=\left(D_{x_{d}}+i \hat{\tau}_{\xi_{d}}-i a \mathrm{Op}_{\top}{ }^{w}\left(h_{k} \underline{\chi}\right)\right), a \in\{+,-\}$, and $R_{1}, R_{1}^{\prime} \in \Psi\left(\lambda_{\top}, \tilde{\tau}, g_{\top}\right)$ and $R_{-M}, R_{-M}^{\prime} \in$ $\Psi\left(\lambda_{\mathrm{T}, \tilde{\tau}}^{-M}, g_{\mathrm{T}}\right)$, for arbitrary large $M \in \mathbb{N}$. 
Proof. In the proof we shall denote by $R_{j}$ a generic operator in $\Psi\left(\lambda_{\mathrm{T}, \tilde{\tau}}^{j}, g_{\mathrm{T}}\right), j \in \mathbb{R}$, whose expression may change from one line to the other.

Observe that we have, for any $M \in \mathbb{N}$,

$$
M_{k} \Xi=M_{k} \mathrm{Op}_{\mathrm{T}}\left(\underline{\chi}^{2} \Xi+R_{-M}=\mathrm{Op}_{\mathrm{T}}\left(m_{k} \underline{\chi}^{2}\right) \Xi+(1+\gamma \varepsilon) R_{1} \Xi+R_{-M} .\right.
$$

With Lemma 4.27 applied with $\mathscr{U}_{\delta}$, a conic neighborhood of $\operatorname{supp}(\underline{\chi})$, we have

$$
\mathrm{Op}_{\mathrm{T}}\left(m_{k} \underline{\chi}^{2}\right)=\mathrm{Op}_{\mathrm{T}}\left(h_{k} \underline{\chi}\right)^{2} \bmod \Psi\left((1+\gamma \varepsilon) \lambda_{\mathrm{T}, \tilde{\tau}}, g_{\mathrm{T}}\right),
$$

using the properties of the tangential calculus (see Proposition 4.3). This yields

$$
\begin{aligned}
M_{k} & \Xi=\mathrm{Op}_{\top}\left(h_{k} \underline{\chi}\right)^{2} \Xi+(1+\gamma \varepsilon) R_{1} \Xi+R_{-M} \\
& =\mathrm{Op}_{\top}{ }^{w}\left(h_{k} \underline{\chi}\right)^{2} \Xi+(1+\gamma \varepsilon) R_{1} \Xi+R_{-M} .
\end{aligned}
$$

We then find

$$
\begin{aligned}
Q_{k} \Xi= & \left(D_{x_{d}}+i \hat{\tau}_{\xi_{d}}\right)^{2} \Xi+M_{k} \Xi \\
= & \left(D_{x_{d}}+i \hat{\tau}_{\xi_{d}}+i \mathrm{Op}^{w}\left(h_{k} \underline{\underline{\chi}}\right)\right)\left(D_{x_{d}}+i \hat{\tau}_{\xi_{d}}-i \mathrm{Op}^{w}\left(h_{k} \underline{\chi}\right)\right) \Xi \\
& +i\left[D_{x_{d}}+i \hat{\tau}_{\xi_{d}}, \mathrm{Op}^{w}{ }^{w}\left(h_{k} \underline{\underline{\chi}}\right)\right] \Xi+(1+\gamma \varepsilon) R_{1} \Xi+R_{-M},
\end{aligned}
$$

In fact, the order of the operators can be changed and we find

$$
\begin{aligned}
Q_{k} \Xi= & \left(D_{x_{d}}+i \hat{\tau}_{\xi_{d}}-i \mathrm{Op}^{w}{ }^{w}\left(h_{k} \underline{\chi}\right)\right)\left(D_{x_{d}}+i \hat{\tau}_{\xi_{d}}+i \mathrm{Op}^{w}{ }^{w}\left(h_{k} \underline{\chi}\right)\right) \Xi \\
& -i\left[D_{x_{d}}+i \hat{\tau}_{\xi_{d}}, \mathrm{Op}^{w}{ }^{w}\left(h_{k} \underline{\chi}\right)\right] \Xi+(1+\gamma \varepsilon) R_{1} \Xi+R_{-M} .
\end{aligned}
$$

The following lemma then yields the result.

Lemma 4.29. Let $\chi=\chi_{0}$ or $\chi_{1}$ and, accordingly, $\Xi=\Xi_{0}$ or $\Xi_{1}$. We have, for $a \in\{+,-\}$, $\left[D_{x_{d}}+i \hat{\tau}_{\xi_{d}}, Q_{k, a}\right] \Xi=-i a\left[D_{x_{d}}+i \hat{\tau}_{\xi_{d}}, \mathrm{Op}_{\mathrm{T}}{ }^{w}\left(h_{k} \underline{\chi}\right)\right] \Xi=(1+\gamma \varepsilon) R_{1} \Xi+R_{-M}$ with $R_{1} \in \Psi\left(\lambda_{\mathrm{T}, \tilde{\tau}}, g_{\mathrm{T}}\right)$ and $R_{-M} \in \Psi\left(\lambda_{\mathrm{T}, \tilde{\tau}}^{-M}, g_{\mathrm{T}}\right)$.

Proof. We have $\left[\hat{\tau}_{\xi_{d}}, \mathrm{Op}_{\mathrm{T}}{ }^{w}\left(h_{k} \chi\right)\right] \in \Psi\left((1+\gamma \varepsilon) \lambda_{\mathrm{T}, \tilde{\tau}}, g_{\mathrm{T}}\right)$ as a consequence of the tangential calculus we have introduced. We have $\left[D_{x_{d}}, \mathrm{Op}^{w}{ }^{w}\left(h_{k} \underline{\chi}\right)\right]=\mathrm{Op}^{w}{ }^{w}\left(D_{x_{d}}\left(h_{k} \underline{\chi}\right)\right)$. We then write

$$
D_{x_{d}}\left(h_{k} \underline{\chi}\right)=D_{x_{d}}\left(h_{k}\right) \underline{\chi}+h_{k} D_{x_{d}}(\underline{\chi}) \text {. }
$$

Because of the definition of $\underline{\chi}$ we have $D_{x_{d}} \underline{\chi}\left(\varrho^{\prime}\right) \equiv 0$ in $\operatorname{supp}\left(\chi\left(\varrho^{\prime}\right)\right)$. Thus Op $_{\mathrm{T}}{ }^{w}\left(\left(D_{x_{d}} \underline{\chi}\right) h_{k}\right) \Xi \in$ $\Psi\left(\lambda_{\mathrm{T}, \tilde{\tau}}^{-M}, g_{\mathrm{T}}\right)$, for any $M \in \mathbb{N}$. Next, by Lemma 4.27 we have $\underline{\chi} D_{x_{d}} h_{k} \in S\left((1+\varepsilon \gamma) \lambda_{\mathrm{T}, \tilde{\tau}}, g_{\mathrm{T}}\right)$, which concludes the proof.

Lemma 4.30. Let $\chi=\chi_{0}$ or $\chi_{1}$ and, accordingly, $\Xi=\Xi_{0}$ or $\Xi_{1}$. Let $k, \ell \in\{1,2\}$ and $a, b \in\{+,-\}$. We have, for any $M \in \mathbb{N}$,

$$
\left[Q_{k, a}, Q_{\ell, b}\right] \Xi=(1+\gamma \varepsilon) R_{1} \Xi+R_{-M}, \quad\left[D_{x_{d}}+i \hat{\tau}_{\xi_{d}}, Q_{k, a} Q_{\ell, b}\right] \Xi=(1+\gamma \varepsilon) R_{1,1} \Xi+R_{1,-M},
$$

with $R_{1} \in \Psi\left(\lambda_{\mathrm{T}, \tilde{\tau}}, g_{\mathrm{T}}\right), R_{1,1} \in \Psi_{\tilde{\tau}}^{1,1}, R_{-M} \in \Psi_{\tilde{\tau}}^{0,-M}$, and $R_{1,-M} \in \Psi_{\tilde{\tau}}^{1,-M}$.

Proof. Since $\left[\mathrm{Op}_{\mathrm{T}}{ }^{w}\left(h_{k} \chi\right), \mathrm{Op}_{T^{w}}{ }^{w}\left(h_{l} \chi\right)\right] \in \Psi\left((1+\gamma \varepsilon) \lambda_{\mathrm{T}, \tilde{\tau}}, g_{\mathrm{T}}\right)$, using the properties of the tangential calculus (see Proposition 4.3), the result follows from Lemma 4.29.

We may now provide a proof of the microlocal estimate for the region $\mathcal{E}_{0}$.

Proof of Proposition 4.25. In the proof, we shall denote by $R_{j, k}$ a generic operator in $\Psi_{\tilde{\tau}}^{j, k}, j \in \mathbb{N}$, $k \in \mathbb{R}$, whose expression may change from one line to the other. We denote by $M$ an arbitrarily large integer whose value may change from one line to the other. 
With the previous lemmata we write, using that $\chi_{1} \equiv 1$ on $\operatorname{supp}\left(\chi_{0}\right)$,

$$
\begin{aligned}
P_{\varphi} \Xi_{0} & =Q_{1} Q_{2} \Xi_{0}=Q_{1} \Xi_{1} Q_{2} \Xi_{0}+R_{4,-M} \\
& =Q_{1,-} Q_{1,+} \Xi_{1} Q_{2,-} Q_{2,+} \Xi_{0}+(1+\gamma \varepsilon) R_{2,1} \Xi_{0}+R_{4,-M} \\
& =Q_{1,-} Q_{1,+} Q_{2,-} Q_{2,+} \Xi_{0}+(1+\gamma \varepsilon) R_{2,1} \Xi_{0}+R_{4,-M} \\
& =Q_{1,-} Q_{1,+} Q_{2,-} \Xi_{1} Q_{2,+} \Xi_{0}+(1+\gamma \varepsilon) R_{2,1} \Xi_{0}+R_{4,-M} \\
& =Q_{1,-} Q_{2,-} Q_{1,+} \Xi_{1} Q_{2,+} \Xi_{0}+(1+\gamma \varepsilon) R_{2,1} \Xi_{0}+R_{4,-M} \\
& =Q^{-} Q^{+} \Xi_{0}+(1+\gamma \varepsilon) R_{2,1} \Xi_{0}+R_{4,-M},
\end{aligned}
$$

with $Q^{-}=Q_{1,-} Q_{2,-}$ and $Q^{+}=Q_{1,+} Q_{2,+}$.

The principal symbol of $Q^{-}$is $q^{-}=q_{1-} q_{2,-} \in S_{\tilde{\tau}}^{2,0}$ in a conic neighborhood of $\operatorname{supp}\left(\chi_{0}\right)$, where all the roots of $q^{-}$have negative imaginary parts. Thus,

$$
\text { the operator } Q^{-} \text {fulfills the requirements of Lemma B.1. }
$$

For both $Q_{1,+}$ and $Q_{2,+}$ we have the following estimate, characterized by the loss of a half derivative and a trace observation, as given by Lemma B.6, for $\ell, m \in \mathbb{R}$,

$$
\gamma^{1 / 2}\left\|\tilde{\tau}^{m-1 / 2} \Xi_{0} v\right\|_{1, \ell, \tilde{\tau}} \lesssim\left\|\tilde{\tau}^{m} Q_{k,+} \Xi_{0} v\right\|_{0, \ell, \tilde{\tau}}+\left|\operatorname{tr}\left(\tilde{\tau}^{m} \Xi_{0} v\right)\right|_{0, \ell+1 / 2, \tilde{\tau}}+\|v\|_{0,-M, \tilde{\tau}}, \quad k=1,2,
$$

for $v \in \mathscr{S}\left(\overline{\mathbb{R}_{+}^{N}}\right)$, and for $\tau \geq \tau_{*}$ and $\gamma \geq 1$ chosen sufficiently large, and $\varepsilon \in[0,1]$. Then, according to Proposition B.7, applied with $\alpha_{1}=\alpha_{2}=1$, we have the following estimate for the operator $Q^{+}$, for $M>0$ and $\ell \in \mathbb{R}$,

$$
\gamma\left\|\tilde{\tau}^{-1} \Xi_{0} v\right\|_{2, \ell, \tilde{\tau}}+\left|\operatorname{tr}\left(\Xi_{0} v\right)\right|_{1, \ell+1 / 2, \tilde{\tau}} \lesssim\left\|Q^{+} \Xi_{0} v\right\|_{0, \ell, \tilde{\tau}}+\left|\operatorname{tr}\left(\Xi_{0} v\right)\right|_{1, \ell+1 / 2, \tilde{\tau}}+\|v\|_{2,-M, \tilde{\tau}},
$$

for $v \in \mathscr{S}\left(\overline{\mathbb{R}_{+}^{N}}\right)$, and for $\tau$ and $\gamma$ chosen sufficiently large.

With (4.46) and (4.47), applying now Proposition B.8, and using that, for any $M \in \mathbb{N},\left[D_{x_{d}}+\right.$ $\left.i \hat{\tau}_{\xi_{d}}, Q^{+}\right] \Xi_{1}=(1+\gamma \varepsilon) R_{1,1} \Xi_{1}+R_{1,-M}$ by Lemma 4.30, we obtain

$$
\gamma\left\|\tilde{\tau}^{-1} \Xi_{0} v\right\|_{4,0, \tilde{\tau}}+\left|\operatorname{tr}\left(\Xi_{0} v\right)\right|_{3,1 / 2, \tilde{\tau}} \lesssim\left\|Q^{-} Q^{+} \Xi_{0} v\right\|_{+}+\left|\operatorname{tr}\left(\Xi_{0} v\right)\right|_{1,5 / 2, \tilde{\tau}}+\|v\|_{4,-M, \tilde{\tau}}
$$

for $v \in \mathscr{S}\left(\overline{\mathbb{R}_{+}^{N}}\right)$, and for $\tau \geq \tau_{*}$ and $\gamma \geq 1$ chosen sufficiently large, for $\varepsilon \in\left[0, \varepsilon_{1}\right]$ with $\varepsilon_{1}>0$ chosen sufficiently small. Finally, with (4.45), we conclude the proof of Proposition 4.25 by choosing $\gamma$ large and $\varepsilon \in\left[0, \varepsilon_{2}\right]$ with $\varepsilon_{2}>0$ chosen sufficiently small.

Remark 4.31. Note that the end of the proof of Proposition 4.25 is a point where the introduction of the second large parameter $\gamma$ is crucial. Even in the case $\varepsilon=0$, that is for a weight function that only depend on the variable $z_{N}$, taking $\gamma$ large is needed to conclude.

4.8. Microlocal estimate in the region $F$. In the region $F$ we have $\tilde{\tau} \lesssim \lambda_{\mathrm{T}, \tilde{\tau}}$ and the symbols of the operators $Q_{k}$ are characterized by two simple roots that are separated (see the first item of Proposition 4.21). We prove the following estimate.

Proposition 4.32. Let $M \in \mathbb{N}$. There exist $\tau_{0} \geq \tau_{*}, \gamma_{0} \geq 1$, and $C>0$ such that

$$
\left\|\Xi_{F} v\right\|_{4,0, \tilde{\tau}}+\left|\operatorname{tr}\left(\Xi_{F} v\right)\right|_{3,1 / 2, \tilde{\tau}} \leq C\left(\left\|P_{\varphi} \Xi_{F} v\right\|_{+}+\left|\operatorname{tr}\left(\Xi_{F} v\right)\right|_{1,5 / 2, \tilde{\tau}}+\|v\|_{4,-M, \tilde{\tau}}\right),
$$

for $\tau \geq \tau_{0}, \gamma \geq \gamma_{0}, \varepsilon \in[0,1]$, and for $v \in \mathscr{S}\left(\overline{\mathbb{R}_{+}^{N}}\right)$.

Proof. We write $\chi_{0}=\chi_{F}$ and $\Xi_{0}=\Xi_{F}$, to ease the reading of the proof.

We also let $\chi_{1}, \underline{\chi} \in S\left(1, g_{\mathrm{T}}\right)$ be supported in $\mathcal{M}_{\mathrm{T}, V}$, homogeneous of degree zero, and be such that $\left|\hat{\tau}\left(\varrho^{\prime}\right)\right| \leq \frac{1}{2} \theta_{0} \lambda_{\mathrm{T}, \tilde{\tau}}\left(\varrho^{\prime}\right)$ in their support (using the notation of Section 4.4) and such that $\chi_{1} \equiv 1$ on a conic neighborhood of $\operatorname{supp}\left(\chi_{0}\right)$ and $\underline{\chi} \equiv 1$ on a conic neighborhood of $\operatorname{supp}\left(\chi_{1}\right)$. This can be 
done as follows, for instance for the construction of $\chi_{1}$. We introduce $V_{1} \subset V$ an open set of $\mathbb{R}^{N}$ that is a neighborhood of $\operatorname{supp}\left(\chi_{V_{0}}\right)$ in $\overline{\mathbb{R}_{+}^{N}}$, in particular $V_{0} \Subset V_{1}$ (the local geometry is illustrated in Figure 4) and we choose $\chi_{V_{1}} \in \mathscr{C}^{\infty}\left(\overline{\mathbb{R}_{+}^{N}}\right)$ such that

$$
\chi_{V_{1}} \equiv 1 \text { on a neighborhood of } V_{1}, \quad \operatorname{supp}\left(\chi_{V_{1}}\right) \subset V .
$$

We set

$$
\chi_{1}\left(\varrho^{\prime}\right)=\chi_{V_{1}}(z) \chi_{4, F} \in S\left(1, g_{T}\right),
$$

with the function $\chi_{\delta, F}$ as introduced in Section 4.4. We have $\left|\hat{\tau}\left(\varrho^{\prime}\right)\right| \leq \frac{1}{8} \theta_{0} \lambda_{T, \tilde{\tau}}\left(\varrho^{\prime}\right)$, which leaves "enough room" for a similar construction for $\chi$. We set $\Xi_{1}=\mathrm{Op}_{\mathrm{T}}\left(\chi_{1}\right)$.

With Proposition 4.21, in a conic neighborhood of $\operatorname{supp}(\chi)$ the roots of $q_{k}, k=1,2$, are simple, and we may write

$$
q_{k}(\varrho)=q_{k,+}(\varrho) q_{k,-}(\varrho), \quad q_{k, \pm}(\varrho)=\xi_{d}-\rho_{k, \pm}\left(\varrho^{\prime}\right),
$$

where $\rho_{k, \pm} \in S\left(\lambda_{\mathrm{T}, \tilde{\tau}}, g_{\mathrm{T}}\right)$ in a conic neighborhood of $\operatorname{supp}(\underline{\chi})$ and there we have

$$
\operatorname{Im} \rho_{k,+} \geq C \lambda_{\mathrm{T}, \tilde{\tau}}, \quad \operatorname{Im} \rho_{k,-} \leq-C \lambda_{\mathrm{T}, \tilde{\tau}} .
$$

We set $Q_{k, \pm}=D_{x_{d}}-\mathrm{Op}_{\mathrm{T}}{ }^{w}\left(\underline{\chi} \rho_{k, \pm}\right)$.

In the proof we shall denote by $R_{j, k}$ as a generic operator in $\Psi_{\tilde{\tau}}^{j, k}, j \in \mathbb{N}, k \in \mathbb{R}$, whose expression may change from one line to the other.

Lemma 4.33. Let $\Xi=\Xi_{0}$ or $\Xi_{1}$. We have, for arbitrary large $M \in \mathbb{N}$,

$$
\begin{aligned}
Q_{k} \Xi & =Q_{k,+} Q_{k,-} \Xi+\gamma R_{1,0} \Xi+R_{2,-M} \\
& =Q_{k,-} Q_{k,+} \Xi+\gamma R_{1,0} \Xi+R_{2,-M} .
\end{aligned}
$$

Proof. We have

$$
Q_{k,+} Q_{k,-}=D_{x_{d}}^{2}-\left(\mathrm{Op}_{\mathrm{T}}{ }^{w}\left(\underline{\chi} \rho_{k,+}\right)+\mathrm{Op}_{\mathrm{T}}{ }^{w}\left(\underline{\chi} \rho_{k,-}\right)\right) D_{x_{d}}+\mathrm{Op}_{\mathrm{T}}{ }^{w}\left(\underline{\chi} \rho_{k,+}\right) \mathrm{Op}_{\mathrm{T}}{ }^{w}\left(\underline{\chi} \rho_{k,-}\right)+\gamma R_{0,1} .
$$

We thus find, for any $M \in \mathbb{N}$,

$$
\begin{aligned}
Q_{k,+} Q_{k,-} \Xi= & \left(\mathrm{Op}^{w}(\underline{\chi}) D_{x_{d}}^{2}-\left(\mathrm{Op}_{\mathrm{T}}{ }^{w}\left(\underline{\chi} \rho_{k,+}\right)+\mathrm{Op}^{w}\left(\underline{\chi} \rho_{k,-}\right)\right) D_{x_{d}}+\mathrm{Op}^{w}{ }^{w}\left(\underline{\chi} \rho_{k,+}\right) \mathrm{Op}^{w}\left(\underline{\chi} \rho_{k,-}\right)\right) \Xi \\
& +\gamma R_{1,0} \Xi+R_{2,-M} \\
= & \mathrm{Op}^{w}\left(\underline{\chi} q_{k}\right) \Xi+\gamma R_{1,0} \Xi+R_{2,-M} \\
= & Q_{k} \Xi+\gamma R_{1,0} \Xi+R_{2,-M} .
\end{aligned}
$$

This result yields, for any $M \in \mathbb{N}$,

$$
\begin{aligned}
P_{\varphi} \Xi_{0} & =Q_{1} Q_{2} \Xi_{0}=Q_{1} \Xi_{1} Q_{2} \Xi_{0}+R_{4,-M} \\
& =Q_{1,-} Q_{1,+} \Xi_{1} Q_{2,-} Q_{2,+} \Xi_{0}+\gamma R_{3,0} \Xi_{0}+R_{4,-M} \\
& =Q_{1,-} Q_{1,+} Q_{2,-} Q_{2,+} \Xi_{0}+\gamma R_{3,0} \Xi_{0}+R_{4,-M} \\
& =Q^{-} Q^{+}+\gamma R_{3,0} \Xi_{0}+R_{4,-M},
\end{aligned}
$$

where $Q^{-}=Q_{1,-} Q_{2,-}$ and $Q^{+}=Q_{1,+} Q_{2,+}$.

Both roots of the symbol $q^{-}$of the operator $Q^{-}$are in the lower half complex plane in a conic neighborhood of $\operatorname{supp}\left(\chi_{0}\right)$. Then, with Lemma B.1 we have the following perfect elliptic estimate, for any $M>0$,

$$
\left\|\Xi_{0} v\right\|_{2,0, \tilde{\tau}}+\left|\operatorname{tr}\left(\Xi_{0} v\right)\right|_{1,1 / 2, \tilde{\tau}} \lesssim\left\|Q^{-} \Xi_{0} v\right\|_{+}+\|v\|_{2,-M, \tilde{\tau}},
$$

for $v \in \mathscr{S}\left(\overline{\mathbb{R}_{+}^{N}}\right)$, for $\tau \geq \tau_{*}$ and $\gamma \geq 1$ chosen sufficiently large, and $\varepsilon \in[0,1]$. 
The roots of the first-order factor $Q_{k,+}, k=1$ or 2 , are in upper half complex plane. Then, with Lemma B.4, we have the following elliptic estimate, yet with a trace observation term in the r.h.s., for $M>0$ and $\ell \in \mathbb{R}$,

$$
\left\|\mid \Xi_{0} v\right\|_{1, \ell, \tilde{\tau}} \leq C\left(\left\|Q_{k,+} \Xi_{0} v\right\|_{0, \ell, \tilde{\tau}}+\left|\operatorname{tr}\left(\Xi_{0} v\right)\right|_{0, \ell+1 / 2, \tilde{\tau}}+\|v\|_{0,-M, \tilde{\tau}}\right),
$$

for $v \in \mathscr{S}\left(\overline{\mathbb{R}_{+}^{N}}\right)$, for $\tau \geq \tau_{*}$ and $\gamma \geq 1$ chosen sufficiently large, and $\varepsilon \in[0,1]$. Then, according to Proposition B.7, applied with $\alpha_{1}=\alpha_{2}=0$ and $\delta_{1}=\delta_{2}=0$, we have the following estimates for the operator $Q^{+}$, for $M>0$ and $\ell \in \mathbb{R}$,

$$
\left\|\Xi_{0} v\right\|_{2, \ell, \tilde{\tau}}+\left|\operatorname{tr}\left(\Xi_{0} v\right)\right|_{1, \ell+1 / 2, \tilde{\tau}} \lesssim\left\|Q^{+} \Xi_{0} v\right\|_{0, \ell, \tilde{\tau}}+\left|\operatorname{tr}\left(\Xi_{0} v\right)\right|_{1, \ell+1 / 2, \tilde{\tau}}+\|v\|_{2,-M, \tilde{\tau}},
$$

for $v \in \mathscr{S}\left(\overline{\mathbb{R}_{+}^{N}}\right)$, and for $\tau \geq \tau_{*}$ and $\gamma \geq 1$ chosen sufficiently large.

Applying now Proposition B.8, with (4.49) and (4.50), we obtain

$$
\left\|\Xi_{0} v\right\|_{4,0, \tilde{\tau}}+\left|\operatorname{tr}\left(\Xi_{0} v\right)\right|_{3,1 / 2, \tilde{\tau}} \lesssim\left\|Q^{-} Q^{+} \Xi_{0} v\right\|_{+}+\left|\operatorname{tr}\left(\Xi_{0} v\right)\right|_{1,5 / 2, \tilde{\tau}}+\|v\|_{4,-M, \tilde{\tau}},
$$

for $v \in \mathscr{S}\left(\overline{\mathbb{R}_{+}^{N}}\right)$, and for $\tau \geq \tau_{*}$ and $\gamma \geq 1$ chosen sufficiently large, for $\varepsilon \in[0,1]$. Finally, with (4.48), we conclude the proof of Proposition 4.32 by choosing $\tau$ and $\gamma$ large.

4.9. Proof of the Carleman estimate of Theorem 4.17. We choose $W$ an open neighborhood of $z_{0}$ in $\mathbb{R}^{N}$ such that $W \Subset V_{0}$ (see Figure 4). Let $u=w_{\mid Z}$, with $w \in \mathscr{C}_{c}^{\infty}\left(\left(0, S_{0}\right) \times \mathbb{R}^{d}\right)$ and $\operatorname{supp}(w) \subset W$. We set $v=e^{\tau \varphi} u$.

We collect the different estimations that we have obtained in Propositions 4.24, 4.25, and 4.32. For some $\delta=\delta_{0} \in(0,1)$ to be kept fixed, for $\tau_{0} \geq \tau_{*}, \gamma_{0} \geq 1$, and $\varepsilon_{0} \in(0,1]$ we have

$$
\gamma^{\frac{1}{2}}\left\|\tilde{\tau}^{-1 / 2} \Xi_{\delta,-}^{(k)} v\right\|_{4,0, \tilde{\tau}}+\left|\operatorname{tr}\left(\Xi_{\delta,-}^{(k)} v\right)\right|_{3,1 / 2, \tilde{\tau}} \lesssim\left\|P_{\varphi} \Xi_{\delta,-}^{(k)} v\right\|_{+}+\left|\operatorname{tr}\left(\Xi_{\delta,-}^{(k)} v\right)\right|_{0,7 / 2, \tilde{\tau}}+\|v\|_{4,-M, \tilde{\tau}},
$$

for $k=1,2$, and

$$
\gamma\left\|\tilde{\tau}^{-1} \Xi_{\delta, 0} v\right\|_{4,0, \tilde{\tau}}+\left|\operatorname{tr}\left(\Xi_{\delta, 0} v\right)\right|_{3,1 / 2, \tilde{\tau}} \lesssim\left\|P_{\varphi} \Xi_{\delta, 0} v\right\|_{+}+\left|\operatorname{tr}\left(\Xi_{\delta, 0} v\right)\right|_{1,5 / 2, \tilde{\tau}}+\|v\|_{4,-M, \tilde{\tau}},
$$

and

$$
\left\|\Xi_{F} v\right\|_{4,0, \tilde{\tau}}+\left|\operatorname{tr}\left(\Xi_{F} v\right)\right|_{3,1 / 2, \tilde{\tau}} \lesssim\left\|P_{\varphi} \Xi_{F} v\right\|_{+}+\left|\operatorname{tr}\left(\Xi_{F} v\right)\right|_{1,5 / 2, \tilde{\tau}}+\|v\|_{4,-M, \tilde{\tau}},
$$

for $\tau \geq \tau_{0}, \gamma \geq \gamma_{0}, \varepsilon \in\left[0, \varepsilon_{0}\right]$.

We then pick $\alpha>0$ meant to be chosen small in what follows, and we shall consider $\alpha((4.51)+$ $(4.53))+(4.52)$. We will choose $\tau$ sufficiently large so that $\alpha \tau^{1 / 2} \geq 1$.

We first note that we have the following lemma whose proof is provided below.

Lemma 4.34. There exists $C>0$ such that

$$
\alpha \gamma^{\frac{1}{2}} \sum_{k=1,2}\left\|\tilde{\tau}^{-1 / 2} \Xi_{\delta,-}^{(k)} v\right\|_{4,0, \tilde{\tau}}+\gamma\left\|\tilde{\tau}^{-1} \Xi_{\delta, 0} v\right\|_{4,0, \tilde{\tau}}+\alpha\left\|\Xi_{F} v\right\|_{4,0, \tilde{\tau}} \geq C \gamma\left\|\tilde{\tau}^{-1} v\right\|_{4,0, \tilde{\tau}},
$$

for $\tau$ chosen sufficiently large.

With a similar, yet simpler, proof, we have the following lemma.

Lemma 4.35. We have

$$
\alpha \sum_{k=1,2}\left|\operatorname{tr}\left(\Xi_{\delta,-}^{(k)} v\right)\right|_{3,1 / 2, \tilde{\tau}}+\left|\operatorname{tr}\left(\Xi_{\delta, 0} v\right)\right|_{3,1 / 2, \tilde{\tau}}+\alpha\left|\operatorname{tr}\left(\Xi_{F} v\right)\right|_{3,1 / 2, \tilde{\tau}} \gtrsim \alpha|\operatorname{tr}(v)|_{3,1 / 2, \tilde{\tau}},
$$

for $\tau$ chosen sufficiently large. 
With these two lemmata we obtain

$$
\begin{aligned}
\gamma\left\|\tilde{\tau}^{-1} v\right\|_{4,0, \tilde{\tau}}+\alpha\left(\left\|\Xi_{F} v\right\|_{4,0, \tilde{\tau}}\right. & \left.+\gamma^{\frac{1}{2}} \sum_{k=1,2}\left\|\tilde{\tau}^{-1 / 2} \Xi_{\delta,-}^{(k)} v\right\|_{4,0, \tilde{\tau}}\right) \\
& +\alpha|\operatorname{tr}(v)|_{3,1 / 2, \tilde{\tau}} \lesssim \alpha(\text { r.h.s.(4.51) + r.h.s. }(4.53))+\text { r.h.s.(4.52). }
\end{aligned}
$$

The next lemma is crucial in the computation of the commutator $\left[P_{\varphi}, \Xi_{\delta, 0}\right]$. A proof is given below.

Lemma 4.36. We have $\left[P_{\varphi}, \Xi_{\delta, 0}\right]=\operatorname{Op}(g)+\mathrm{Op}(h)+\gamma^{2} R_{3,-1}$, where $g, h \in \gamma \Psi_{\tilde{\tau}}^{3,0}$ and $R_{3,-1} \in \Psi_{\tilde{\tau}}^{3,-1}$, with

- $g(\varrho)=0$ for $z$ in a neighborhood of $V_{0}$

- $h(\varrho)=\sum_{j=0}^{3} h_{j}\left(\varrho^{\prime}\right) \xi_{d}^{j}, h_{j} \in \gamma \Psi\left(\lambda_{\mathrm{T}, \tilde{\tau}}^{3-j}, g_{\mathrm{T}}\right)$, homogeneous of degree $3-j$, and $\chi_{\delta,-}^{(1)}+\chi_{\delta,-}^{(2)}+$ $\chi_{F} \geq 1$ in a conic neighborhood of $\operatorname{supp}\left(h_{j}\right)$ in the variables $(\zeta, \tau, \gamma, \varepsilon)$, for $z \in V_{0}$, $j=0, \ldots, 3$.

We have $\left[P_{\varphi}, \Xi\right] \in \gamma R_{3,0}$, for $\Xi=\Xi_{\delta,-}^{(1)}, \Xi_{\delta,-}^{(2)}$ or $\Xi_{F}$. Lemma 4.36 gives, for any $M \in \mathbb{N}$, $\|\mathrm{Op}(g) v\|_{+} \lesssim\|v\|_{3,-M, \tilde{\tau}}$, and we obtain

$$
\alpha \sum_{k=1,2}\left\|P_{\varphi} \Xi_{\delta,-}^{(k)} v\right\|_{+}+\left\|P_{\varphi} \Xi_{\delta, 0} v\right\|_{+}+\alpha\left\|P_{\varphi} \Xi_{F} v\right\|_{+} \lesssim\left\|P_{\varphi} v\right\|_{+}+\|\mathrm{Op}(h) v\|_{+}+\alpha \gamma\|v\|_{3,0, \tilde{\tau}}+\gamma^{2}\|v\|_{3,-1, \tilde{\tau}} .
$$

From (4.54) and (4.51)-(4.53) we thus obtain, for $\alpha$ chosen sufficiently small (and kept fixed for the remainder of the proof) and $\tau$ chosen sufficiently large

$$
\begin{aligned}
\gamma\left\|\tilde{\tau}^{-1} v\right\|_{4,0, \tilde{\tau}}+\left\|\Xi_{F} v\right\|_{4,0, \tilde{\tau}}+\gamma^{\frac{1}{2}} \sum_{k=1,2} & \left\|\tilde{\tau}^{-1 / 2} \Xi_{\delta,-}^{(k)} v\right\|_{4,0, \tilde{\tau}} \\
& +|\operatorname{tr}(v)|_{3,1 / 2, \tilde{\tau}} \lesssim\left\|P_{\varphi} v\right\|_{+}+|\operatorname{tr}(v)|_{1,5 / 2, \tilde{\tau}}+\|\mathrm{Op}(h) v\|_{+} .
\end{aligned}
$$

We set $\chi=\chi_{\delta,-}^{(1)}+\chi_{\delta,-}^{(2)}+\chi_{F}$. We have the following lemma whose proof is given below.

Lemma 4.37. Let $W$ be an open set of $\mathbb{R}^{N}$ with $W \Subset V_{0}$. There exist $C>0$ and $\tau_{1} \geq \tau_{*}$ such that $\left\|\mathrm{Op}_{\mathrm{T}}\left(h_{j}\right) w\right\|_{+} \leq C \gamma\left(\left\|\mathrm{Op}_{\mathrm{T}}(\chi) w\right\|_{0,3-j, \tilde{\tau}}+\gamma(1+\varepsilon \gamma)\|w\|_{0,2-j, \tilde{\tau}}\right)$, for $w \in \mathscr{S}\left(\overline{\mathbb{R}_{+}^{N}}\right), \operatorname{supp}(w) \subset W$ and $\tau \geq \tau_{1}$.

Thus, we obtain

$$
\|\mathrm{Op}(h) v\|_{+} \leq \sum_{j=0}^{3}\left\|\mathrm{Op}\left(h_{j}\right) D_{x_{d}}^{j} v\right\|_{+} \lesssim \sum_{j=0}^{3} \gamma\left\|\mathrm{Op}_{\mathrm{T}}(\chi) D_{x_{d}}^{j} v\right\|_{0,3-j, \tilde{\tau}}+\gamma(1+\varepsilon \gamma)\|v\|_{3,-1, \tilde{\tau}}
$$

As $\left[\operatorname{Op}_{T}(\chi), D_{x_{d}}^{j}\right] \in \gamma \Psi_{\tilde{\tau}}^{j-1,0}$ we obtain

$$
\begin{aligned}
\|\mathrm{Op}(h) v\|_{+} & \lesssim \gamma\left\|\mathrm{Op}_{\mathrm{T}}(\chi) v\right\|_{3,0, \tilde{\tau}}+\gamma^{2}\|v\|_{3,-1, \tilde{\tau}} \\
& \lesssim \gamma\left(\sum_{k=1,2}\left\|\Xi_{\delta,-}^{(k)} v\right\|_{3,0, \tilde{\tau}}+\left\|\Xi_{F} v\right\|_{3,0, \tilde{\tau}}\right)+\gamma^{2}\|v\|_{3,-1, \tilde{\tau}} .
\end{aligned}
$$

Using this estimate in (4.55), for $\tau$ chosen sufficiently large, we thus obtain

$$
\gamma\left\|\tilde{\tau}^{-1} v\right\|_{4,0, \tilde{\tau}}+\left\|\Xi_{F} v\right\|_{4,0, \tilde{\tau}}+\gamma^{\frac{1}{2}} \sum_{k=1,2}\left\|\tilde{\tau}^{-1 / 2} \Xi_{\delta,-}^{(k)} v\right\|_{4,0, \tilde{\tau}}+|\operatorname{tr}(v)|_{3,1 / 2, \tilde{\tau}} \lesssim\left\|P_{\varphi} v\right\|_{+}+|\operatorname{tr}(v)|_{1,5 / 2, \tilde{\tau}} .
$$

The end of the proof of Theorem 4.17 is then classical. 
Proof of Lemma 4.34. With Lemma 4.14 we may write

$$
\begin{aligned}
X & =\alpha \gamma^{\frac{1}{2}} \sum_{k=1,2}\left\|\tilde{\tau}^{-1 / 2} \Xi_{\delta,-}^{(k)} v\right\|_{4,0, \tilde{\tau}}+\gamma\left\|\tilde{\tau}^{-1} \Xi_{\delta, 0} v\right\|_{4,0, \tilde{\tau}}+\alpha\left\|\Xi_{F} v\right\|_{4,0, \tilde{\tau}} \\
& \gtrsim \sum_{j=0}^{4}\left(\alpha \gamma^{\frac{1}{2}} \sum_{k=1,2}\left\|\tilde{\tau}^{-1 / 2} \Lambda_{\mathrm{T}, \tilde{\tau}}^{4-j} D_{x_{d}}^{j} \Xi_{\delta,-}^{(k)} v\right\|_{+}+\gamma\left\|\tilde{\tau}^{-1} \Lambda_{\mathrm{T}, \tilde{\tau}}^{4-j} D_{x_{d}}^{j} \Xi_{\delta, 0} v\right\|_{+}+\alpha\left\|\Lambda_{\mathrm{T}, \tilde{\tau}}^{4-j} D_{x_{d}}^{j} \Xi_{F} v\right\|_{+}\right)
\end{aligned}
$$

yielding

$$
X \gtrsim \gamma \sum_{j=0}^{4}\left(\sum_{k=1,2}\left\|\tilde{\tau}^{-1} \Lambda_{\mathrm{T}, \tilde{\tau}}^{4-j} D_{x_{d}}^{j} \Xi_{\delta,-}^{(k)} v\right\|_{+}+\left\|\tilde{\tau}^{-1} \Lambda_{\mathrm{T}, \tilde{\tau}}^{4-j} D_{x_{d}}^{j} \Xi_{\delta, 0} v\right\|_{+}+\left\|\tilde{\tau}^{-1} \Lambda_{\mathrm{T}, \tilde{\tau}}^{4-j} D_{x_{d}}^{j} \Xi_{F} v\right\|_{+}\right),
$$

as $\alpha \geq \alpha \gamma^{\frac{1}{2}} \tilde{\tau}^{-1 / 2} \geq \gamma \tilde{\tau}^{-1}$ using, on the one hand, that $(\tau \varphi)^{-1 / 2}=\gamma^{\frac{1}{2}} \tilde{\tau}^{-1 / 2} \leq 1$ since $\tau \geq \tau_{*} \geq 1$ and $\varphi \geq 1$, and, on the other hand, that $\alpha \tau^{1 / 2} \geq 1$ implies $\alpha \tilde{\tau}^{1 / 2}=\alpha(\tau \gamma \varphi)^{1 / 2} \geq \gamma^{1 / 2}$ since $\varphi \geq 1$. We then find, with $h=\chi_{\delta,-}^{(1)}+\chi_{\delta,-}^{(2)}+\chi_{\delta, 0}+\chi_{F} \in S\left(1, g_{\mathrm{T}}\right), X \gtrsim \gamma \sum_{j=0}^{4} \| \tilde{\tau}^{-1} \Lambda_{\mathrm{T}, \tilde{\tau}}^{4-j} D_{x_{d}}^{j}$ Op $\mathrm{p}_{\mathrm{T}}(h) v \|_{+}$. As $\left[D_{x_{d}}^{j}, \mathrm{Op}_{\mathrm{T}}(h)\right] \in \gamma \Psi_{\tilde{\tau}}^{j-1,0}$, we obtain

$$
X+\gamma^{2}\left\|\tilde{\tau}^{-1} v\right\|_{3,0, \tilde{\tau}} \gtrsim \gamma \sum_{j=0}^{4}\left\|\tilde{\tau}^{-1} \Lambda_{\mathrm{T}, \tilde{\tau}}^{4-j} \mathrm{Op}_{\mathrm{T}}(h) D_{x_{d}}^{j} v\right\|_{+} .
$$

By the (local) Gårding inequality of Proposition 4.15, as $h\left(\varrho^{\prime}\right) \geq 1$ in a neighborhood of $V_{0} \cap \overline{\mathbb{R}_{+}^{N}}$ that contains $\operatorname{supp}(v)$, we obtain

$$
X+\gamma^{2}\left\|\tilde{\tau}^{-1} v\right\|_{3,0, \tilde{\tau}} \gtrsim \gamma \sum_{j=0}^{4}\left\|\tilde{\tau}^{-1} D_{x_{d}}^{j} v\right\|_{0,4-j, \tilde{\tau}} \asymp \gamma\left\|\tilde{\tau}^{-1} v\right\|_{4,0, \tilde{\tau}},
$$

We conclude by taking $\tau$ sufficiently large with the usual semi-classical inequality (4.17).

Proof of Lemma 4.36. Up to $\gamma^{2} S_{\tilde{\tau}}^{3,-1}$, the principal symbol of $\left[P_{\varphi}, \Xi_{\delta, 0}\right]$ is given by $-i\left\{p_{\varphi}, \chi_{\delta, 0}\right\}$, and thus involves derivatives of $\chi_{\delta, 0}$. We recall the form of $\chi_{\delta, 0}$, as introduced in Section 4.4,

$$
\chi_{\delta, 0}\left(\varrho^{\prime}\right)=\chi_{V_{0}}(z)\left(1-\chi_{1 / 4, F}\left(\varrho^{\prime}\right)\right) \chi_{0}\left(\mu_{1}\left(\varrho^{\prime}\right) / \delta\right) \chi_{0}\left(\mu_{2}\left(\varrho^{\prime}\right) / \delta\right) .
$$

Computing $-i\left\{p_{\varphi}, \chi_{\delta, 0}\right\}$, we obtain the following list of terms.

Terms involving derivatives of $\chi_{V_{0}}(z)$ : Those terms contribute to the symbol $g$ that vanishes in a neighborhood of $V_{0}$.

Terms involving derivatives of $\chi_{\mathbf{1 / 4 , F}}\left(\varrho^{\prime}\right)$ : Those terms are supported in $\left\{\theta_{1} \lambda_{\mathrm{T}, \tilde{\tau}} / 8 \leq \tilde{\tau} \leq\right.$ $\left.\theta_{1} \lambda_{\mathrm{T}, \tilde{\tau}} / 4\right\}$, using the notation of Section 4.4. As $\chi_{1, F}=1$ for $\tilde{\tau} \leq \theta_{1} \lambda_{\mathrm{T}, \tilde{\tau}} / 2$, we see that $\chi_{F}\left(\varrho^{\prime}\right)=\chi_{V_{0}}(z) \chi_{1, F}\left(\varrho^{\prime}\right)=1$ in a neighborhood of the support of those terms for $z \in V_{0}$. Those terms contribute to the symbol $h$.

Terms involving derivatives of $\chi_{\mathbf{0}}\left(\mu_{\boldsymbol{k}}\left(\varrho^{\prime}\right) / \delta\right), \boldsymbol{k}=\mathbf{1 , 2}$ : From the definition of $\chi_{0}$ we see that those terms are supported in $\left\{-3 \leq \mu_{k}\left(\varrho^{\prime}\right) / \delta \leq-2\right\}$. We have $\chi_{-}\left(\mu_{k}\left(\varrho^{\prime}\right) / \delta\right)=1$ in a conic neighborhood of this set. As $\chi_{1, F}\left(\varrho^{\prime}\right)+\left(1-\chi_{1 / 4, F}\left(\varrho^{\prime}\right)\right) \geq 1$ we find that $\chi_{F}\left(\varrho^{\prime}\right)+\chi_{\delta,-}^{(k)}\left(\varrho^{\prime}\right) \geq 1$ in the support of those terms if $z \in V_{0}$. Those terms contribute to the symbol $h$.

Proof of Lemma 4.37. Let $\chi_{W}(z) \in \mathscr{C}_{c}^{\infty}\left(V_{0}\right)$ be such that $\chi_{W} \equiv 1$ in a neighborhood of $W$. The microlocal version of the Gårding inequality of Proposition 4.16 gives, by Lemma 4.36,

$$
\operatorname{Re}\left(\mathrm{Op}_{\mathrm{T}}(\chi) \text { Ор } \mathrm{T}\left(\chi_{W} h_{j}\right) w, \mathrm{Op}_{\mathrm{T}}\left(\chi_{W} h_{j}\right) w\right)_{+}+\|w\|_{0,-M, \tilde{\tau}}^{2} \gtrsim\left\|\mathrm{Op}_{\mathrm{T}}\left(\chi_{W} h_{j}\right) w\right\|_{+}^{2} \text {. }
$$

Then, with the Young inequality, we obtain

$$
\| \mathrm{Op}_{\mathrm{T}}(\chi) \text { Ор }\left(\chi_{W} h_{j}\right) w\left\|_{+}+\right\| w\left\|_{0,-M, \tilde{\tau}}, \gtrsim\right\| \mathrm{Op}_{\mathrm{T}}\left(\chi_{W} h_{j}\right) w \|_{+} .
$$


Since $\mathrm{Op}_{\mathrm{T}}\left(\chi_{W} h_{j}\right) w=\mathrm{Op}_{\mathrm{T}}\left(h_{j}\right) w+R_{0,-M} w$, with $R_{0,-M} \in \Psi_{\tilde{\tau}}^{0,-M}$, for any $M \in \mathbb{N}$, we obtain

$$
\| \text { Ор } \mathrm{p}_{\mathrm{T}}(\chi) \mathrm{Op}_{\mathrm{T}}\left(h_{j}\right) w\left\|_{+}+\right\| w\left\|_{0,-M, \tilde{\tau}}, \gtrsim\right\| \mathrm{Op}_{\mathrm{T}}\left(h_{j}\right) w \|_{+} .
$$

As $\left[\mathrm{Op}_{\mathrm{T}}(\chi), \mathrm{Op}_{\mathrm{T}}\left(h_{j}\right)\right] \in \gamma(1+\varepsilon \gamma) \Psi\left(\lambda_{\mathrm{T}, \tilde{\tau}}^{2-j}, g_{\mathrm{T}}\right)$, we obtain the sought estimate.

\section{SPectral ineQuality AND APPLicATion}

We start this section by stating and proving an interpolation type inequality. Next, we prove the spectral inequality of Theorem 1.3. Finally, as an application, we state a null-controllability result that follows from it.

5.1. An interpolation inequality. Let $S_{0}>0$ and $\alpha \in\left(0, S_{0} / 2\right)$. We recall the notation $Z=$ $\left(0, S_{0}\right) \times \Omega$ and we introduce $Y=\left(\alpha, S_{0}-\alpha\right) \times \Omega$ for some $\alpha>0$. As is done in other sections, we denote by $z=(s, x) \in Z$, with $s \in\left(0, S_{0}\right)$ and $x \in \Omega$. We recall that $P$ denotes the augmented elliptic operator $P:=D_{s}^{4}+B$, where $B=\Delta_{x}^{2}$.

Theorem 5.1 (Interpolation inequality). Let $\mathscr{O}$ be a nonempty open subset of $\Omega$. There exist $C>0$ and $\delta \in(0,1)$ such that for $u \in H^{4}(Z)$ that satisfies

$$
\left.u(s, x)\right|_{x \in \partial \Omega}=0,\left.\quad \partial_{\nu} u(s, x)\right|_{x \in \partial \Omega}=0, \quad s \in\left(0, S_{0}\right),
$$

we have

$$
\|u\|_{H^{3}(Y)} \leq C\|u\|_{H^{3}(Z)}^{1-\delta}\left(\|P u\|_{L^{2}(Z)}+\sum_{0 \leq j \leq 3}\left\|\partial_{s}^{j} u_{\mid s=0}\right\|_{H^{3-j}(\mathscr{O})}\right)^{\delta} .
$$

First, we provide a local interpolation estimate in a neighborhood of a point of $\{0\} \times \mathscr{O}$.

Lemma 5.2 (local interpolation near $s=0$ ). Let $x_{0} \in \mathscr{O}$, there exist $V$ a neighborhood of $\left(0, x_{0}\right)$ in $\mathbb{R} \times \mathbb{R}^{d}, C>0$, and $\delta \in(0,1)$ such that for $u \in H^{4}(Z)$ we have

$$
\|u\|_{H^{3}(V \cap Z)} \leq C\|u\|_{H^{3}(Z)}^{1-\delta}\left(\|P u\|_{L^{2}(Z)}+\sum_{0 \leq j \leq 3}\left\|\partial_{s}^{j} u_{\mid s=0}\right\|_{H^{3-j}(\mathscr{O})}\right)^{\delta} .
$$

Second, we provide an interpolation estimate with an interior observation, that is, we have an estimate away from the boundary $0 \times \Omega$.

Proposition 5.3 (Interpolation with an interior observation). Let $\mathscr{Z}$ be a nonempty open set in $Z$. There exist $C>0$ and $\delta \in(0,1)$ such that for $u \in H^{4}(Z)$ that satisfies

$$
\left.u(s, x)\right|_{x \in \partial \Omega}=0,\left.\quad \partial_{\nu} u(s, x)\right|_{x \in \partial \Omega}=0, \quad s \in\left(0, S_{0}\right),
$$

we have

$$
\|u\|_{H^{3}(Y)} \leq C\|u\|_{H^{3}(Z)}^{1-\delta}\left(\|P u\|_{L^{2}(Z)}+\|u\|_{L^{2}(\mathscr{Z})}\right)^{\delta} .
$$

With these two local interpolation results, whose proofs are given below, we can then write a proof of Theorem 5.1.

Proof of Theorem 5.1. Introducing $V$ as given in Lemma 5.2, we let $\mathscr{Z}$ be an open subset of $V \cap Z$. With Lemma 5.2 we then have

$$
\|P u\|_{L^{2}(Z)}+\|u\|_{H^{3}(\mathscr{Z})} \leq C\|u\|_{H^{3}(Z)}^{1-\delta}\left(\|P u\|_{L^{2}(Z)}+\sum_{0 \leq j \leq 3}\left\|\partial_{S}^{j} u_{\mid s=0}\right\|_{H^{3-j}(\mathscr{O})}\right)^{\delta},
$$

as we can assume that $\|P u\|_{L^{2}(Z)} \leq\|u\|_{H^{3}(Z)}$ otherwise estimate (5.1) is trivial. Applying Proposition 5.3 we have, for some $\delta^{\prime} \in(0,1)$,

$$
\|u\|_{H^{3}(Y)} \leq C\|u\|_{H^{3}(Z)}^{1-\delta^{\prime}}\left(\|P u\|_{L^{2}(Z)}+\|u\|_{L^{2}(\mathscr{Z})}\right)^{\delta^{\prime}} .
$$

This, with (5.4), gives (5.1) with $\delta^{\prime} \delta$ in place of $\delta$. 
For the proofs of Lemma 5.2 and Proposition 5.3. We shall need the following lemma whose proof can be found in [Rob95].

Lemma 5.4. Let $A \geq 0, B \geq 0$, and $C \geq 0$. We assume that $A \leq B$ and that there exist $\tau_{0}>0$, $\mu>0$ and $v>0$ such that

$$
A \leq e^{-\nu \tau} B+e^{\mu \tau} C, \quad \text { for } \tau \geq \tau_{0} .
$$

Then $A \leq K B^{1-\delta} C^{\delta}$, where $K=\max \left(2, e^{\mu \tau_{0}}\right)$ and $\delta=v /(v+\mu) \in(0,1)$.

Proof of Lemma 5.2. Let $r>0$ and $z_{0}=\left(-r, x_{0}\right)$, where $r$ is chosen sufficiently small to have $B \cap\{s=0\} \subset \mathscr{O}$ with $B=B\left(z_{0}, 4 r\right)$. Let $\psi=-\left|z-z_{0}\right|^{2}$, with $z=(s, x)$. We have $\partial_{s} \psi(z) \leq-C<0$ in $B$. We set $\varphi(z)=e^{\gamma \psi(z)}$. Let $\chi \in \mathscr{C}_{0}^{\infty}\left(\mathbb{R}^{d+1}\right)$ be such that $\chi(z)=1$ if $\left|z-z_{0}\right| \leq 7 r / 2$ and $\chi(z)=0$ if $\left|z-z_{0}\right| \geq 15 r / 4$. We apply the local Carleman estimate of Corollary 3.2 to $v=\chi u$, and we obtain, for $\gamma \geq 1$ chosen sufficiently large (to be kept fixed in what follows),

$$
\sum_{|\alpha| \leq 3} \tau^{7 / 2-|\alpha|}\left\|e^{\tau \varphi} D_{z}^{\alpha} v\right\|_{L^{2}(B \cap Z)} \lesssim\left\|e^{\tau \varphi} P v\right\|_{L^{2}(Z)}+\tau^{1 / 2} \sum_{j=0}^{3}\left|\operatorname{tr}\left(e^{\tau \varphi} D_{s}^{j} v_{\mid s=0^{+}}\right)\right|_{0,3-j, \tau} .
$$

Note that if $\gamma$ is fixed we have $\tau \asymp \tilde{\tau}$. In $\{0\} \times \mathscr{O}$, we have $\varphi \leq e^{-\gamma r^{2}}$ then

$$
\tau^{1 / 2} \sum_{j=0}^{3}\left|\operatorname{tr}\left(e^{\tau \varphi} D_{s}^{j} v_{\mid s=0^{+}}\right)\right|_{0,3-j, \tau} \lesssim e^{C_{3} \tau} \sum_{j=0}^{3}\left|D_{s}^{j} u_{\mid s=0^{+}}\right|_{H^{3-j}(\mathscr{O})}, \quad C_{3}=(1+a) e^{-\gamma r^{2}},
$$

for any $a>0$. We have $P v=\chi P u+[P, \chi] u$. The term $[P, \chi]$ is a differential operator of order 3 and it is supported in $\left\{z \in \mathbb{R}^{d+1} ; 7 r / 2 \leq\left|z-z_{0}\right| \leq 15 r / 4\right\}$. On this set, we have $\varphi \leq e^{-\gamma(7 r / 2)^{2}}$. We thus find

$$
\left\|e^{\tau \varphi}[P, \chi] u\right\|_{L^{2}(Z)} \lesssim e^{C_{1} \tau}\|u\|_{H^{3}(Z)}, \quad C_{1}=e^{-\gamma(7 r / 2)^{2}} .
$$

In $Z$, we have $\varphi \leq e^{-\gamma r^{2}}<C_{3}$; this implies

$$
\left\|e^{\tau \varphi} \chi P u\right\|_{L^{2}(Z)} \lesssim e^{C_{3} \tau}\|P u\|_{L^{2}(Z)} .
$$

In $\left\{z \in \mathbb{R}^{d+1} ;\left|z-z_{0}\right| \leq 3 r\right\}, \chi \equiv 1$ thus $u=v$, and on this set $\varphi \geq e^{-\gamma(3 r)^{2}}$ then we have

$$
e^{C_{2} \tau}\|u\|_{H^{3}\left(B\left(z_{0}, 3 r\right) \cap Z\right)} \lesssim \sum_{|\alpha| \leq 3} \tau^{7 / 2-|\alpha|}\left\|e^{\tau \varphi} D_{z}^{|\alpha|} \nu\right\|_{L^{2}(B \cap Z)}, \quad C_{2}=e^{-\gamma(3 r)^{2}} .
$$

Remark that $C_{1}<C_{2}<C_{3}$, for $a>0$ chosen sufficiently small. Following (5.6)-(5.10) we obtain

$$
\|u\|_{H^{3}\left(B\left(z_{0}, 3 r\right) \cap Z\right)} \lesssim e^{\left(C_{3}-C_{2}\right) \tau}\left(\|P u\|_{L^{2}(Z)}+\sum_{j=0}^{3}\left|D_{s}^{j} u_{\mid s=0^{+}}\right|_{H^{3-j}(\mathscr{O})}\right)+e^{-\left(C_{2}-C_{1}\right) \tau}\|u\|_{H^{3}(Z)} .
$$

Applying Lemma 5.4, we obtain the result with $V=B\left(z_{0}, 3 r\right)$.

We prove Proposition 5.3 by means of two lemmata. For $\alpha^{\prime} \in(0, \alpha)$ and $a \in(0,1)$, we set

$$
Y_{\alpha^{\prime}, a}=\left(\alpha^{\prime}, S_{0}-\alpha^{\prime}\right) \times \Omega_{a},
$$

where $\Omega_{a}=\{x \in \Omega, \operatorname{dist}(x, \partial \Omega)>a>0\}$.

Lemma 5.5. Let $\mathscr{Z}$ be a nonempty open set in $Z$. Let $\alpha^{\prime} \in(0, \alpha)$ and $a \in(0,1)$. There exist $C>0$ and $\delta \in(0,1)$ such that for $u \in H^{4}(Z)$,

$$
\|u\|_{H^{3}\left(Y_{\alpha^{\prime}, a}\right)} \leq C\|u\|_{H^{3}(Z)}^{1-\delta}\left(\|P u\|_{L^{2}(Z)}+\|u\|_{L^{2}(\mathscr{Z})}\right)^{\delta} .
$$


Lemma 5.6. Let $\left(s_{0}, x_{0}\right) \in\left(0, S_{0}\right) \times \partial \Omega$. There exist $\delta \in(0,1), C>0, V_{0}$ a neighborhood of $\left(s_{0}, x_{0}\right), \alpha^{\prime} \in(0, \alpha)$, and $a \in(0,1)$ such that we have

$$
\|u\|_{H^{3}\left(V_{0} \cap Z\right)} \leq C\|u\|_{H^{3}(Z)}^{1-\delta}\left(\|P u\|_{L^{2}(Z)}+\|u\|_{H^{1}\left(Y_{\alpha^{\prime}, a}\right)}\right)^{\delta},
$$

for $u \in H^{4}(Z)$ satisfying

$$
\left.u(s, x)\right|_{x \in \partial \Omega}=0,\left.\quad \partial_{\nu} u(s, x)\right|_{x \in \partial \Omega}=0, \quad s \in\left(0, S_{0}\right) .
$$

Proof of Proposition 5.3. We can assume that $\|P u\|_{L^{2}(Z)} \leq\|u\|_{H^{3}(Z)}$, otherwise inequality (5.3) is obvious. In particular, if (5.3) holds for a value $\delta=\delta_{0}>0$ the estimate also holds for all $\delta \in\left[0, \delta_{0}\right]$ possibly with a larger constant $C=C_{\delta}$. The same observation can be made for the estimations (5.12) and (5.13).

With a compactness argument we can find a finite number of open sets $V_{j}, j \in J$, where estimate (5.13) holds for some values $\delta=\delta_{j} \in(0,1), \alpha_{j}^{\prime} \in(0, \alpha)$, and $a_{j} \in(0,1)$, and such that

$$
\left(\alpha, S_{0}-\alpha\right) \times \partial \Omega \subset \cup_{j \in J} V_{j} .
$$

For $a \in(0,1)$ and $\alpha^{\prime} \in(0, \alpha)$, set $\tilde{Y}_{\alpha^{\prime}, a}=\left(\alpha^{\prime}, S_{0}-\alpha^{\prime}\right) \times \tilde{\Omega}_{a}$, where $\tilde{\Omega}_{a}=\{x \in \Omega, \operatorname{dist}(x, \partial \Omega)<a\}$. There exists $a_{1} \in(0,1)$ and $\alpha_{1} \in(0, \alpha)$ such that $\tilde{Y}_{\alpha_{1}, a_{1}} \subset Z \cap\left(\cup_{j \in J} V_{j}\right)$. Applying the local interpolation estimate (5.13) for each $V_{j}$, using now

$$
\delta_{1}=\min _{j \in J} \delta_{j} \in(0,1), \quad \alpha_{2}=\min _{j \in J} \alpha_{j}^{\prime} \in(0, \alpha), \quad \text { and } a_{2}=\min _{j \in J} a_{j} \in(0,1)
$$

(note that the set $Y_{\alpha^{\prime}, a}$ increases as $\alpha^{\prime}$ and $a$ decrease) we obtain

$$
\|u\|_{H^{3}\left(\tilde{Y}_{\alpha_{1}, a_{1}}\right)} \lesssim\|u\|_{H^{3}(Z)}^{1-\delta_{1}}\left(\|P u\|_{L^{2}(Z)}+\|u\|_{H^{3}\left(Y_{\alpha_{2}, a_{2}}\right)}\right)^{\delta_{1}} .
$$

Let $\mathscr{Z}$ be a nonempty open set in $Z$. By Lemma 5.5 we obtain, for some $\delta_{2} \in(0,1)$,

$$
\|P u\|_{L^{2}(Z)}+\|u\|_{H^{3}\left(Y_{\alpha_{2}, a_{2}}\right)} \lesssim\|u\|_{H^{3}(Z)}^{1-\delta_{2}}\left(\|P u\|_{L^{2}(Z)}+\|u\|_{L^{2}(\mathscr{Z})}\right)^{\delta_{2}},
$$

as the estimate of $\|P u\|_{L^{2}(Z)}$ is clear here. Then, estimates (5.14) and (5.15) give

$$
\|u\|_{H^{3}\left(\tilde{Y}_{\alpha_{1}, a_{1}}\right)} \lesssim\|u\|_{H^{3}(Z)}^{1-\delta_{1} \delta_{2}}\left(\|P u\|_{L^{2}(Z)}+\|u\|_{L^{2}(\mathscr{Z})}\right)^{\delta_{1} \delta_{2}} .
$$

Taking $a \in\left(0, a_{1}\right)$ and $\alpha^{\prime} \in(0, \alpha)$, we have $Y \subset Y_{\alpha^{\prime}, a} \cup \tilde{Y}_{\alpha_{1}, a_{1}}$, and, by (5.12) in Lemma 5.5 and (5.16), we obtain (5.3).

Proof of Lemma 5.5. By a compactness argument, it suffices to prove (5.12) with $B(z, R)$ in place of $Y_{\alpha^{\prime}, a}$ where $z \in \overline{Y_{\alpha^{\prime}, a}}$ and $0<R \leq \min \left(\alpha^{\prime}, a\right) / 2$, implying $B(z, R) \subset Z$. Let $z^{(0)}$ be in $\mathscr{Z}$ and $r_{0}>0$ such that $B\left(z^{(0)}, r_{0}\right) \Subset \mathscr{Z}$. As $Y_{\alpha^{\prime}, a}$ is connected, there exists a path $\Gamma \subset Y_{\alpha^{\prime}, a}$ from $z^{(0)}=\Gamma(0)$ to $z=\Gamma(1)$. Set $r_{1}=\operatorname{dist}(\Gamma, \partial Z)$. We have $r_{1}>0$ by compactness.

Setting now $r=\inf \left(R, r_{0}, r_{1} / 4\right)$, we define a sequence $\left(z^{(j)}\right)_{j}$, for $j \geq 0$, by $z^{(j)}=\Gamma\left(t_{j}\right)$ where $t_{0}=0$ and

$$
t_{j}=\left\{\begin{array}{ll}
\inf A_{j} & \text { if } A_{j} \neq \emptyset, \\
1 & \text { if } A_{j}=\emptyset,
\end{array} \quad A_{j}=\left\{\sigma \in\left(t_{j-1}, 1\right] ; \Gamma(\sigma) \notin B\left(z^{j-1}, r\right)\right\} .\right.
$$

The sequence $\left(z^{(j)}\right)_{j}$ is finite by a compactness argument. The construction of the sequence is illustrated in Figure 5. 


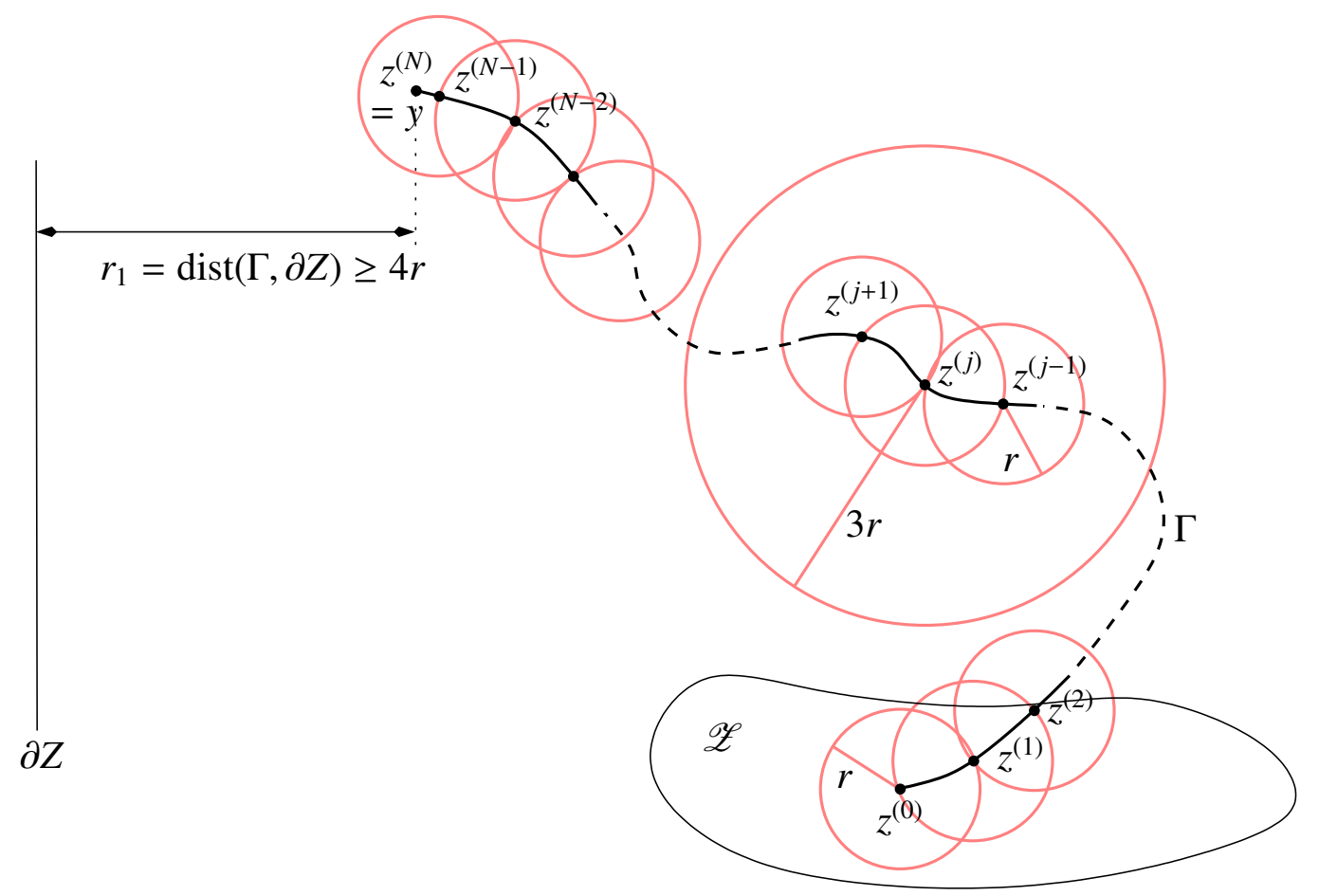

Figure 5. Construction of the sequence $\left(z^{(j)}\right)_{j}, j \in J$, along the path $\Gamma$.

Let $\left(z^{(0)}, \cdots, z^{(N)}\right)$ be such a sequence with $z^{(N)}=z$. Note that we have $B\left(z^{(j+1)}, r\right) \subset B\left(z^{(j)}, 3 r\right) \subset$ $Z$, for $j=0, \cdots, N-1$, because of the choice we made for $r$ above. Now we claim that there exists $C>0$ and $\delta \in(0,1)$ such that

$$
\|u\|_{H^{3}\left(B\left(z^{(j+1)}, r\right)\right)} \leq\|u\|_{H^{3}\left(B\left(z^{(j)}, 3 r\right)\right)} \leq C\|u\|_{H^{3}(Z)}^{1-\delta}\left(\|P u\|_{L^{2}(Z)}+\|u\|_{H^{3}\left(B\left(z^{(j)}, r\right)\right)}\right)^{\delta},
$$

for $j=0, \ldots, N-1$. This claim is proven below.

We assume that $\|P u\|_{L^{2}(Z)} \leq\|u\|_{H^{3}(Z)}$, since otherwise the estimate we wish to prove is obvious. We then have

$$
\|P u\|_{L^{2}(Z)}+\|u\|_{H^{3}\left(B\left(z^{(j+1)}, r\right)\right)} \lesssim\|u\|_{H^{3}(Z)}^{1-\delta}\left(\|P u\|_{L^{2}(Z)}+\|u\|_{H^{3}\left(B\left(z^{(j)}, r\right)\right)}\right)^{\delta} .
$$

By induction on $j$, we find

$$
\|P u\|_{L^{2}(Z)}+\|u\|_{H^{3}(B(z, r))} \lesssim\|u\|_{H^{3}(Z)}^{1-\mu}\left(\|P u\|_{L^{2}(Z)}+\|u\|_{H^{3}\left(B\left(z^{(0)}, r\right)\right)}\right)^{\mu},
$$

where $\mu=\delta^{N}$.

As $P$ is elliptic, and $B\left(z^{(0)}, r\right) \Subset \mathscr{Z}$ we have $\|u\|_{H^{3}\left(B\left(z^{(0)}, r\right)\right)} \lesssim\|P u\|_{L^{2}(Z)}+\|u\|_{L^{2}(\mathscr{Z})}$. This estimate and (5.18) give (5.12).

To prove estimation (5.17) we apply the local Carleman estimate of Proposition 2.7. We set $\psi(z)=-\left|z-z^{(j)}\right|^{2}$ and $\varphi(z)=e^{\gamma \psi(z)}$ and $\chi \in \mathscr{C}_{c}^{\infty}\left(B\left(z^{(j)}, 4 r\right)\right)$ to be such that

$$
\chi(z)= \begin{cases}1 & \text { if } 3 r / 4<\left|z-z^{(j)}\right|<7 r / 2, \\ 0 & \text { if }\left|z-z^{(j)}\right|<5 r / 8 \text { or } 15 r / 4<\left|z-z^{(j)}\right| .\end{cases}
$$


The function $v=\chi u$ is supported in the open set $B\left(z^{(j)}, 4 r\right) \backslash B\left(z^{(j)}, r / 2\right) \subset Z$ where $d \psi$ does not vanish. For $\gamma \geq 1$ chosen sufficiently large, by Proposition 2.7, we have

$$
\sum_{|\alpha| \leq 4} \tau^{3-|\alpha|}\left\|e^{\tau \varphi} D_{z}^{\alpha} v\right\|_{L^{2}(Z)} \lesssim\left\|e^{\tau \varphi} P v\right\|_{L^{2}(Z)} .
$$

We have $P v=\chi P u+[P, \chi] u$ and $[P, \chi]$ is a differential operator of order 3 supported in $A_{1} \cup A_{2}$ with

$$
A_{1}=\left\{z ; 5 r / 8 \leq\left|z-z^{(j)}\right| \leq 3 r / 4\right\}, \quad A_{2}=\left\{z ; 7 r / 2 \leq\left|z-z^{(j)}\right| \leq 15 r / 4\right\} .
$$

We write

$$
\left\|e^{\tau \varphi} P v\right\|_{L^{2}(Z)} \leq\left\|e^{\tau \varphi} P u\right\|_{L^{2}\left(B\left(z^{(j)}, 4 r\right)\right)}+\left\|e^{\tau \varphi}[P, \chi] u\right\|_{L^{2}\left(A_{1} \cup A_{2}\right)} .
$$

Since $\varphi$ decreases as $\left|z-z^{(j)}\right|$ increases, we find

$$
\left\|e^{\tau \varphi} P v\right\|_{L^{2}(Z)} \lesssim e^{\tau C_{3}}\|P u\|_{L^{2}(Z)}+e^{\tau C_{3}}\|u\|_{H^{3}\left(B\left(z^{(j)}, r\right)\right)}+e^{\tau C_{1}}\|u\|_{H^{3}(Z)},
$$

where $C_{1}=e^{-\gamma(7 r / 2)^{2}}$ and $C_{3}=e^{-\gamma(5 r / 8)^{2}}$.

As we have $\chi \equiv 1$ on $B\left(z^{(j)}, 3 r\right) \backslash B\left(z^{(j)}, r\right)$ we have

$$
e^{\tau C_{2}}\|u\|_{H^{3}\left(B\left(z^{(j)}, 3 r\right) \backslash B\left(z^{(j)}, r\right)\right)} \leq \sum_{|\alpha| \leq 4} \tau^{3-|\alpha|}\left\|e^{\tau \varphi} D_{z}^{\alpha} v\right\|_{L^{2}(Z)},
$$

where $C_{2}=e^{-\gamma(3 r)^{2}}$. Remark that $C_{1}<C_{2}<C_{3}$.

Inequalities (5.19), (5.20), and (5.21) give

$$
\|u\|_{H^{3}\left(B\left(z^{(j)}, 3 r\right)\right)} \lesssim e^{\tau\left(C_{3}-C_{2}\right)}\left(\|P u\|_{L^{2}(Z)}+\|u\|_{H^{3}\left(B\left(z^{(j)}, r\right)\right)}\right)+e^{-\tau\left(C_{2}-C_{1}\right)}\|u\|_{H^{3}(Z)} .
$$

as the estimate on $B\left(z^{(j)}, r\right)$ is clear with such a r.h.s. if $\tau \geq \tau_{*} \geq 1$. We can optimize this last estimate applying Lemma 5.4, which yields (5.17), and concludes the proof of Lemma 5.5.

Proof of Lemma 5.6. The proof follows the same ideas as that of estimate (5.17) applying the boundary-type local Carleman estimate of Theorem 4.17. We use local coordinates in a bounded neighborhood $V$ in $\mathbb{R}^{N}$ of the point $z_{0}=\left(s_{0}, x_{0}\right)$ of $\left(0, S_{0}\right) \times \partial \Omega$ as introduced in Section 4.2, such that this part of the boundary is locally given by $\left\{z_{N}=x_{d}=0\right\}$ and $Z$ is locally given by $\left\{z_{N}>0\right\}$; coordinates can be chosen to have moreover $z_{0}=\left(z_{0}^{\prime}, 0\right)$, with $z_{0}^{\prime}=0$. We set $z^{(1)}=(0,2 r)$ where $r>0$.

We let $\psi \in \mathscr{C}^{\infty}\left(\mathbb{R}^{N}\right)$ be such that

$$
\psi(z)= \begin{cases}12 r^{2}-\left|z-z^{(1)}\right|^{2} & \text { if }\left|z-z^{(1)}\right| \leq 3 r, \\ r^{2} & \text { if } 4 r \leq\left|z-z^{(1)}\right| .\end{cases}
$$

We have $\psi(z) \geq r^{2}>0,\left\|\psi^{(k)}\right\|_{L^{\infty}}<\infty, k \in \mathbb{N}$, and

$$
\partial_{\nu} \psi(z)=-\partial_{z_{N}} \psi(z)=2\left(z_{N}-2 r\right) \leq-C<0,
$$

for $\left|z-z^{(1)}\right| \leq 3 r$ and $z_{N}=0$. Upon reducing the open neighborhood $V$, the weight function $\psi$ fulfills the requirements listed in (4.2) and (4.20).

We set $\varphi(z)=e^{\gamma \psi_{\varepsilon}(z)}$, where $\psi_{\varepsilon}(z)=\psi\left(\varepsilon z^{\prime}, z_{N}\right)$. According to Theorem 4.17, there exist a neighborhood $W \Subset V$ in $R^{N}$ of $z_{0}, \tau_{0} \geq \tau_{*}, \gamma_{0} \geq 1$, and $\varepsilon_{0} \in(0,1]$ so that the Carleman estimate (4.21) holds for $\tau \geq \tau_{0}, \gamma \geq \gamma_{0}, \varepsilon \in\left(0, \varepsilon_{0}\right]$ and smooth functions supported in $W$. We set $\gamma=\gamma_{0}$ and $\varepsilon=\varepsilon_{0}$. The geometry of the level sets of the weight function is illustrated in Figure 6 .

In connection with the weight function $\psi_{\varepsilon}$, we introduce the following anisotropic norm in $\mathbb{R}^{N}$, that depends on the (now fixed) parameter $\varepsilon$,

$$
|z-y|_{\varepsilon}=\left(\varepsilon^{2}\left|z^{\prime}-y^{\prime}\right|^{2}+\left(z_{N}-y_{N}\right)^{2}\right)^{1 / 2} .
$$

Note that with $\gamma$ and $\varepsilon$ fixed we have $\tau \asymp \tilde{\tau}$. 


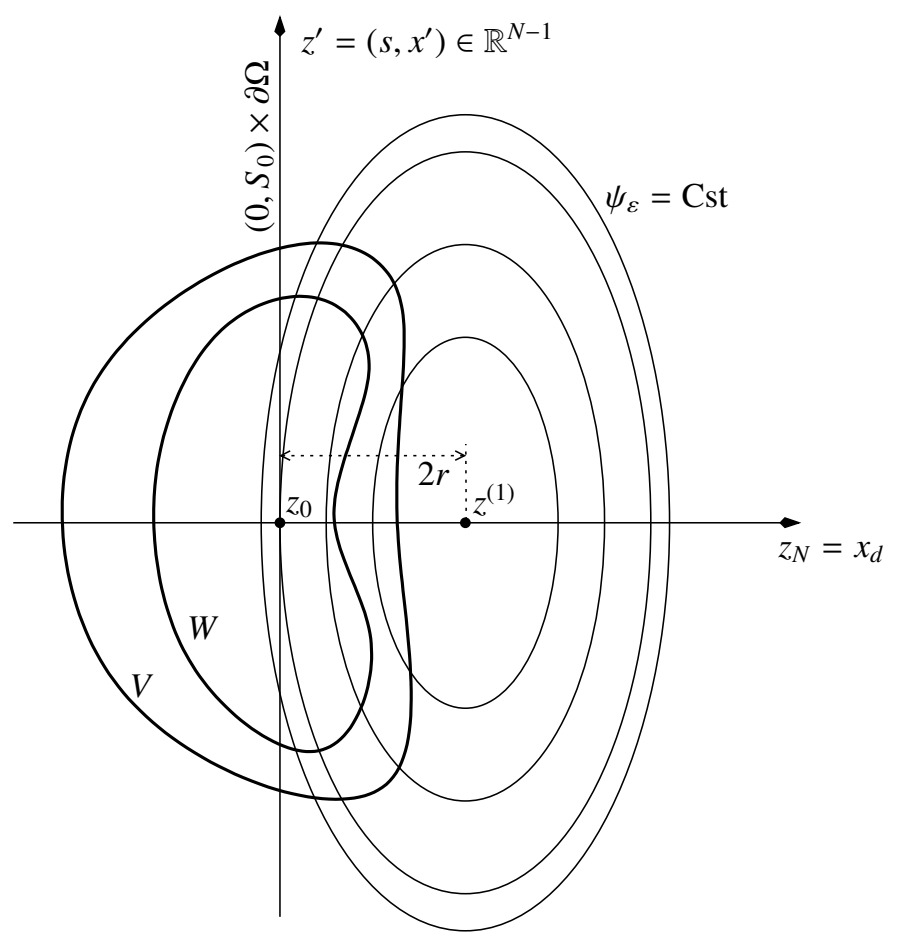

FiguRE 6. Geometry near the boundary for the application of the local Carleman estimate of Theorem 4.17.

We denote by $B_{\varepsilon}(z, r)$ the ball of radius $r$ centered at $z$ associated with this norm. We have

$$
\psi_{\varepsilon}(z)= \begin{cases}12 r^{2}-\left|z-z^{(1)}\right|_{\varepsilon}^{2} & \text { if }\left|z-z^{(1)}\right|_{\varepsilon} \leq 3 r \\ r^{2} & \text { if } 4 r \leq\left|z-z^{(1)}\right|_{\varepsilon} .\end{cases}
$$

Let $\chi_{0} \in \mathscr{C}_{c}^{\infty}(\mathbb{R})$ be such that

$$
\chi_{0}\left(z_{N}\right)= \begin{cases}1 & \text { if }\left|z_{N}\right|<r_{0}, \\ 0 & \text { if } 2 r_{0}<\left|z_{N}\right|,\end{cases}
$$

where $r_{0}<r / 4$. Let also $\chi_{1} \in \mathscr{C}_{c}^{\infty}\left(B_{\varepsilon}\left(z^{(1)}, 3 r\right)\right)$ be such that

$$
\chi_{1}(z)= \begin{cases}1 & \left|z-z^{(1)}\right|_{\varepsilon}<r_{1} \\ 0 & \text { if } r_{1}^{\prime}<\left|z-z^{(1)}\right|_{\varepsilon}\end{cases}
$$

where $r_{1}, r_{1}^{\prime}$ are such that $2 r<r_{1}<r_{1}^{\prime}<3 r$. Observe that if we choose the values of $r_{1}^{\prime}-2 r>0$ and $r_{0}>0$ sufficiently small, then the open set $\left\{z \in Z ; z_{N} \in\left(0,2 r_{0}\right)\right\} \cap\left\{z \in Z ;\left|z-z^{(1)}\right|_{\varepsilon}<r_{1}^{\prime}\right\}$ is contained in $W$. We now set $\chi(z)=\chi_{1}(z) \chi_{0}\left(z_{N}\right)$. Figure 7 shows, near $z_{0}$, the region where $\chi \equiv 1$ and where it varies, that is $\operatorname{supp}\left(\chi^{\prime}\right) \cap Z \subset A_{1} \cup A_{2}$ with

$$
\begin{aligned}
& A_{1}=\left\{z \in Z ; z_{N} \in\left(r_{0}, 2 r_{0}\right) \text { and }\left|z-z^{(1)}\right|_{\varepsilon}<r_{1}^{\prime}\right\}, \\
& A_{2}=\left\{z \in Z ; z_{N} \in\left(0,2 r_{0}\right) \text { and } r_{1}<\left|z-z^{(1)}\right|_{\varepsilon}<r_{1}^{\prime}\right\} .
\end{aligned}
$$




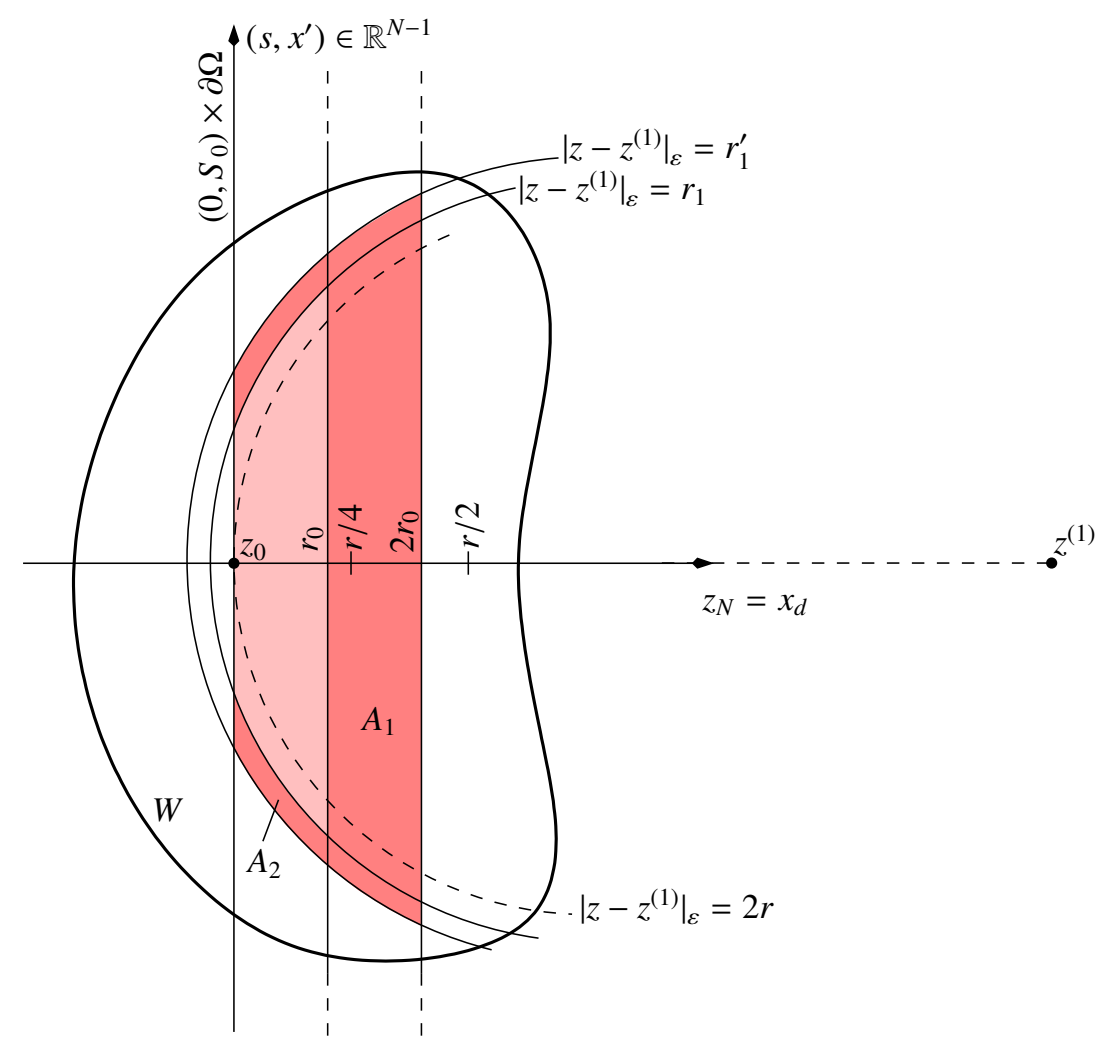

FIGURE 7. Geometry near the boundary for the derivation of the local interpolation inequality. The light color region shows where $\chi \equiv 1$; the dark color region shows where $\chi$ varies. Note that the relative scale of the two axes has been modified, if compared to Figure 6, for a better display of the regions $A_{1}$ and $A_{2}$ near $z_{0}$.

The Carleman estimate (4.17) applies to $v=\chi u$, by a density argument. As $u_{\left.\right|_{N}=0^{+}}=0$ and $\partial_{\nu} u_{z_{N}=0^{+}}=0$ we obtain (the values of $\gamma$ and $\varepsilon$ were fixed above)

$$
\sum_{|\alpha| \leq 3} \tau^{3-|\alpha|}\left\|e^{\tau \varphi} D_{s, x}^{\alpha} v\right\|_{L^{2}(W \cap Z)} \lesssim\left\|e^{\tau \varphi} P v\right\|_{L^{2}(W \cap Z)}, \quad \tau \geq \tau_{0} .
$$

We have $P v=\chi P u+[P, \chi] u$, where $[P, \chi]$ is a differential operator of order 3 that is supported in $A_{1} \cup A_{2}$. On $A_{1}$, we have $\varphi \leq e^{\gamma\left(12 r^{2}-\left(2 r-2 r_{0}\right)^{2}\right)}$. On $A_{2}$ we have $\varphi \leq e^{\gamma\left(12 r^{2}-r_{1}^{2}\right)}$. We thus obtain

$$
\left\|e^{\tau \varphi} P v\right\|_{L^{2}(W \cap Z)} \lesssim e^{\tau C_{3}}\left(\|P u\|_{L^{2}(Z)}+\|u\|_{H^{3}\left(Y_{\alpha^{\prime}, a}\right)}\right)+e^{\tau C_{1}}\|u\|_{H^{3}(Z)},
$$

where $C_{1}=e^{\gamma\left(12 r^{2}-r_{1}^{2}\right)}, C_{3}=e^{\gamma\left(12 r^{2}-\left(2 r-2 r_{0}\right)^{2}\right)}$ and $0<a<r_{0}$ and some $\alpha^{\prime} \in(0, \alpha)$ (recalling the definition of the set $Y_{\alpha^{\prime}, a}$ in (5.11)).

We restrict the 1.h.s. of (5.22) to $V_{0}=\left\{z \in Z ; z_{N} \in\left(0, r_{0}\right)\right\} \cap\left\{z \in Z ;\left|z-z^{(1)}\right|<r_{2}\right\}$, with $r_{2}=r+r_{1} / 2$, whose closure is a neighborhood of $z_{0}$ in $\bar{Z}$. Note that $2 r<r_{2}<r_{1}$. As on this set we have $\varphi \geq e^{\gamma\left(12 r^{2}-r_{2}^{2}\right)}$ and $u \equiv v$, we obtain

$$
e^{\tau C_{2}}\|u\|_{H^{3}\left(V_{0}\right)} \leq \sum_{|\alpha| \leq 3} \tau^{3-|\alpha|}\left\|e^{\tau \varphi} D_{s, x}^{\alpha} v\right\|_{L^{2}(W \cap Z)},
$$


where $C_{2}=e^{\gamma\left(12 r^{2}-\left(r+r_{1} / 2\right)^{2}\right)}$. Then (5.22), (5.23) and (5.24) give

$$
\|u\|_{H^{3}\left(V_{0}\right)} \lesssim e^{\tau\left(C_{3}-C_{2}\right)}\left(\|P u\|_{L^{2}(Z)}+\|u\|_{H^{3}\left(Y_{\alpha^{\prime}, a}\right)}\right)+e^{-\tau\left(C_{2}-C_{1}\right)}\|u\|_{H^{3}(Z)} .
$$

Observe that we have $C_{1}<C_{2}<C_{3}$. By Lemma 5.4, we obtain the sought local interpolation inequality at the boundary.

5.2. Spectral inequality. Let $\phi_{j}$ and $\mu_{j}$ be eigenfunctions and associated eigenvalues of the biLaplace operator $B$ with the clamped boundary conditions, that form a Hilbert basis for $L^{2}(\Omega)$, viz.,

$$
B \phi_{j}=\mu_{j} \phi_{j}, \quad \phi_{j \mid \partial \Omega}=\partial_{\nu} \phi_{j \mid \partial \Omega}=0, \quad\left(\phi_{j}, \phi_{k}\right)_{L^{2}(\Omega)}=\delta_{j k},
$$

with $0<\mu_{0} \leq \mu_{1} \leq \cdots \leq \mu_{j} \leq \cdots$. We now prove the spectral inequality of Theorem 1.3, namely, for some $C>0$,

$$
\|u\|_{L^{2}(\Omega)} \leq C e^{C \mu^{1 / 4}}\|u\|_{L^{2}(\mathscr{O})}, \quad \mu>0, \quad u \in \operatorname{Span}\left\{\phi_{j} ; \mu_{j} \leq \mu\right\} .
$$

Proof. We let $\mu>0$ and we pick $\alpha_{0}, \ldots, \alpha_{n} \in \mathbb{C}$ with $n \in \mathbb{N}$ such that $\mu_{n} \leq \mu<\mu_{n+1}$. We set

$$
u(x)=\sum_{\mu_{j} \leq \mu} \alpha_{j} \phi_{j}(x), \quad w(s, x)=\sum_{\mu_{j} \leq \mu} \alpha_{j} \mu_{j}^{-3 / 4} f\left(\mu_{j}^{1 / 4} s\right) \phi_{j}(x),
$$

where $f(s)=\gamma \sin (\gamma s) \cosh (\gamma s)-\gamma \cos (\gamma s) \sinh (\gamma s)$ where here $\gamma=\sqrt{2} / 2$. As $D_{s}^{4} f=-f$, we have $P v=0$, with $P=D_{s}^{4}+B$. We also have

$$
f(0)=f^{\prime}(0)=f^{(2)}(0)=0, \quad f^{(3)}(0)=1,
$$

and

$$
f(s)=g(\gamma s), \quad g(s)=\frac{1}{2}\left(e^{-s} \cos (s-\pi / 4)-e^{s} \cos (s+\pi / 4)\right) .
$$

Since $\left.w(s, x)\right|_{x \in \partial \Omega}=\left.\partial_{\nu} w(s, x)\right|_{x \in \partial \Omega}=0$, the interpolation inequality of Theorem 5.1 yields

$$
\|w\|_{H^{3}(Y)} \leq C\|w\|_{H^{3}(Z)}^{1-\delta}\left\|\partial_{s}^{3} w_{\mid s=0}\right\|_{L^{2}(\mathscr{O})}^{\delta} .
$$

Observe that we have $\partial_{s}^{3} w_{\mid s=0}=u$ and $\|w\|_{H^{3}(Y)} \gtrsim\|w\|_{L^{2}(Y)}$ with

$$
\begin{aligned}
\|w\|_{L^{2}(Y)}^{2} & =\sum_{\mu_{j} \leq \mu} \mu_{j}^{-3 / 2}\left|\alpha_{j}\right|^{2} \int_{\alpha}^{S_{0}-\alpha} f\left(\mu_{j}^{1 / 4} s\right)^{2} d s=\sum_{\mu_{j} \leq \mu}\left|\alpha_{j}\right|^{2} \gamma^{-1} \mu_{j}^{-7 / 4} \int_{\alpha \gamma \mu_{j}^{1 / 4}}^{\left(S_{0}-\alpha\right) \gamma \mu_{j}^{1 / 4}} g(s)^{2} d s \\
& \gtrsim \mu^{-7 / 4} \sum_{\mu_{j} \leq \mu}\left|\alpha_{j}\right|^{2}=\mu^{-7 / 4}\|u\|_{L^{2}(\Omega)}^{2},
\end{aligned}
$$

using the following lemma, whose proof is given below.

Lemma 5.7. Let $0<a<b$ and $t_{0}>0$. There exists $C_{0}$ such that $\int_{a t}^{b t} g(s)^{2} d s \geq C_{0}$ for $t \geq t_{0}$.

We thus obtain

$$
\|u\|_{L^{2}(\Omega)} \lesssim \mu^{7 / 8}\|w\|_{H^{3}(Z)}^{1-\delta}\|u\|_{L^{2}(\mathscr{O})}^{\delta} .
$$

Next, we estimate $\|w\|_{H^{3}(Z)}$, with the following lemma, which, from (5.27), allows one to conclude the proof of Theorem 1.3.

Lemma 5.8. There exists $C>0$ such that $\|w\|_{H^{3}(Z)} \leq C e^{C \mu^{1 / 4}}\|u\|_{L^{2}(\Omega)}$. 
Proof. We have

$$
\|w\|_{H^{3}(Z)}^{2} \asymp \sum_{k=0}^{3} \int_{0}^{S_{0}}\left\|\partial_{s}^{k} w(s, .)\right\|_{H^{3-k}(\Omega)}^{2} d s \lesssim \sum_{k=0}^{3} \int_{0}^{S_{0}}\left\|\partial_{s}^{k} w(s, .)\right\|_{H^{4}(\Omega)}^{2} d s
$$

where $H^{s}(\Omega)$ denotes the classical Sobolev spaces in $\Omega$. Recalling from (1.15) that, if $v_{\mid \partial \Omega}=$ $\partial_{v} v_{\mid \partial \Omega}=0$, we have $\|v\|_{H^{4}(\Omega)} \lesssim\left\|\Delta^{2} v\right\|_{L^{2}(\Omega)}$, we find

$$
\begin{aligned}
\left\|\partial_{s}^{k} w(s, .)\right\|_{H^{4}(\Omega)}^{2} & \lesssim\left\|\Delta^{2} \sum_{\mu_{j} \leq \mu} \alpha_{j} \mu_{j}^{(k-3) / 4} f^{(k)}\left(\mu_{j}^{1 / 4} s\right) \phi_{j}\right\|_{L^{2}(\Omega)}^{2}=\left\|\sum_{\mu_{j} \leq \mu} \alpha_{j} \mu_{j}^{(k+1) / 4} f^{(k)}\left(\mu_{j}^{1 / 4} s\right) \phi_{j}\right\|_{L^{2}(\Omega)}^{2} \\
& =\sum_{\mu_{j} \leq \mu}\left|\alpha_{j}\right|^{2} \mu_{j}^{(k+1) / 2}\left(f^{(k)}\left(\mu_{j}^{1 / 4} s\right)\right)^{2} \lesssim \mu^{2} e^{S_{0} \mu^{1 / 4}} \sum_{\mu_{j} \leq \mu}\left|\alpha_{j}\right|^{2} .
\end{aligned}
$$

Integrating this estimate over $\left(0, S_{0}\right)$ and summing over $k$ yields the result.

Proof of Lemma 5.7. For $s \in[-\pi / 2+2 k \pi, 2 k \pi], k \in \mathbb{N}^{*}$, we have $\cos (s+\pi / 4) \geq \sqrt{2} / 2$. For $t_{1}$ chosen sufficiently large, if $t \geq t_{1}$, there exists $k \in \mathbb{N}$ such that $[-\pi / 2+2 k \pi, 2 k \pi] \subset[a t, b t]$ and $|g(s)|=\frac{1}{2}\left|e^{-s} \cos (s-\pi / 4)-e^{s} \cos (s+\pi / 4)\right| \geq 1$. Then, $\int_{a t}^{b t} g(s)^{2} d s \geq \pi / 2$. Finally, there exists $C>0$ such that $\int_{a t}^{b t} g(s)^{2} d s \geq C$ for $t \in\left[t_{0}, t_{1}\right]$, since the function $g(s)^{2}$ is almost everywhere positive.

5.3. A null-controllability result for a higher-order parabolic equation. Let $T>0$. We consider here the controlled evolution equation on $(0, T) \times \Omega$ with the clamped boundary conditions $(v$ denotes the outer unit normal to $\partial \Omega$ ):

$$
\partial_{t} y+\Delta^{2} y=\chi_{\mathscr{O}} f, \quad y_{\mid(0, T) \times \partial \Omega}=0, \quad \partial_{\nu} y_{\mid(0, T) \times \partial \Omega}=0, \quad y_{\mid t=0}=y_{0} \in L^{2}(\Omega),
$$

where $\mathscr{O}$ is an open subset of $\Omega$ and $\chi_{\mathscr{O}} \in L^{\infty}(\Omega)$ is such that $\chi_{\mathscr{O}}>0$ on $\mathscr{O}$. The function $f \in L^{2}((0, T) \times \Omega)$ is the control function here. Well-posedness for this parabolic system is recalled in Corollary 1.10. One may wonder if one can choose $f$ to drive the solution from its initial condition $y_{0}$ to zero at final time $T$. Thanks to the spectral inequality of Theorem 1.3 one can answer positively to this null-controllability question.

Theorem 5.9 (Null-controllability). There exists $C>0$ such that for any $y_{0} \in L^{2}(\Omega)$, there exists $f \in L^{2}((0, T) \times \Omega)$ such that the solution to (5.28) vanishes at $T=0$ and moreover $\|f\|_{L^{2}((0, T) \times \Omega)} \leq$ $C\left\|y_{0}\right\|_{L^{2}(\Omega)}$.

The proof can be adapted in a straight forward manner from the proof scheme of [LR95] developed for the heat equation and that is presented in a fairly synthetic way in the survey article [LL12].

\section{RESOLVENT ESTIMATE AND APPLICATION}

Using one of the interpolation inequalities proven in Section 5 (Proposition 5.3), we prove the resolvent estimate of Theorem 1.4. Finally, as an application, we state a stabilization result that follows from it for the plate equation.

6.1. Resolvent estimate. Let $U \in D(\mathcal{B})=\left(H^{4}(\Omega) \cap H_{0}^{2}(\Omega)\right) \times H_{0}^{2}(\Omega)$ and $F \in \mathscr{H}=H_{0}^{2}(\Omega) \times$ $L^{2}(\Omega)$, be such that

$$
\left(i \sigma \operatorname{Id}_{\mathscr{H}}-\mathcal{B}\right) U=F, \quad U={ }^{t}\left(u_{0}, u_{1}\right), \quad F={ }^{t}\left(f_{0}, f_{1}\right),
$$

for $\sigma \neq 0$. Our goal is to find an estimate of the form $\|U\|_{\mathscr{H}} \leq K e^{K|\sigma|^{1 / 2}}\|F\|_{\mathscr{H}}$. We have

$$
i \sigma u_{0}+u_{1}=f_{0}, \quad\left(-\sigma^{2}-i \sigma \alpha+B\right) u_{0}=f, \quad \text { with } f=(i \sigma-\alpha) f_{0}-f_{1} .
$$


Multiplying the second equation by $\bar{u}_{0}$ and an integration over $\Omega$ give

$$
\left\langle\left(-\sigma^{2}+B\right) u_{0}, u_{0}\right\rangle_{L^{2}(\Omega)}-i \sigma\left\|\alpha^{1 / 2} u_{0}\right\|_{L^{2}(\Omega)}^{2}=\left\langle f, u_{0}\right\rangle_{L^{2}(\Omega)},
$$

The first term is real and the second term is purely imaginary. We thus have

$$
\sigma\left\|\alpha^{1 / 2} u_{0}\right\|_{L^{2}(\Omega)}^{2}=-\operatorname{Im}\left\langle f, u_{0}\right\rangle_{L^{2}(\Omega)} .
$$

Using that $\alpha \geq \delta>0$ in $\mathscr{O}$ yields

$$
\delta \sigma_{0}\left\|u_{0}\right\|_{L^{2}(\mathscr{O})}^{2} \leq \sigma\left\|\alpha^{1 / 2} u_{0}\right\|_{L^{2}(\Omega)}^{2} \leq\|f\|_{L^{2}(\Omega)}\left\|u_{0}\right\|_{L^{2}(\Omega)},
$$

for $\sigma \geq \sigma_{0}$.

A key estimate is given by the following lemma. We provide a proof below.

Lemma 6.1. There exists $C>0$ such that

$$
\left\|u_{0}\right\|_{H^{3}(\Omega)} \leq C e^{C|\sigma|^{1 / 2}}\left(\|f\|_{L^{2}(\Omega)}+\left\|u_{0}\right\|_{L^{2}(\mathscr{O})}\right) .
$$

Then estimate (6.3) yields

$$
\left\|u_{0}\right\|_{H^{2}(\Omega)} \lesssim e^{C|\sigma|^{1 / 2}}\left(\|f\|_{L^{2}(\Omega)}+\left\|u_{0}\right\|_{L^{2}(\Omega)}^{\frac{1}{2}}\|f\|_{L^{2}(\Omega)}^{\frac{1}{2}}\right), \quad \sigma \geq \sigma_{0},
$$

and with the Young inequality we obtain

$$
\left\|u_{0}\right\|_{H^{2}(\Omega)} \lesssim e^{C|\sigma|^{1 / 2}}\|f\|_{L^{2}(\Omega)} .
$$

Using the form of $f$ given in (6.2) we then obtain

$$
\left\|u_{0}\right\|_{H^{2}(\Omega)} \lesssim e^{C|\sigma|^{1 / 2}}\left(\left\|f_{0}\right\|_{L^{2}(\Omega)}+\left\|f_{1}\right\|_{L^{2}(\Omega)}\right),
$$

Finally as $u_{1}=f_{0}-i \sigma u_{0}$ we obtain

$$
\left\|u_{0}\right\|_{H^{2}(\Omega)}+\left\|u_{1}\right\|_{L^{2}(\Omega)} \lesssim e^{C|\sigma|^{1 / 2}}\left(\left\|f_{0}\right\|_{L^{2}(\Omega)}+\left\|f_{1}\right\|_{L^{2}(\Omega)}\right),
$$

yielding the resolvent estimate of Theorem 1.4.

6.2. Proof of Lemma 6.1. Let $\rho=\exp (i \operatorname{sgn}(\sigma) \pi / 4)$, yielding $\rho^{2}=\operatorname{sgn}(\sigma) i$ and $\rho^{4}=-1$. We set $u=\exp \left(s \rho|\sigma|^{1 / 2}\right) u_{0}$ and have $Q u=e^{s \rho|\sigma|^{1 / 2}} f$, with $Q=D_{s}^{4}+B+\alpha D_{s}^{2}$, recalling (6.2). Let $S_{0}>0$ and $\beta \in\left(0, S_{0} / 2\right)$. Let also $Z=\left(0, S_{0}\right) \times \Omega$ and $Y=\left(\beta, S_{0}-\beta\right) \times \Omega$. We then apply the interpolation inequality of Proposition 5.3: with $0<\beta_{1}<\beta_{2}<S_{0}$ we have $C>0$ and $\delta_{0}>0$ such that

$$
\|u\|_{H^{3}(Y)} \leq C\|u\|_{H^{3}(Z)}^{1-\delta}\left(\|Q u\|_{L^{2}(Z)}+\|u\|_{L^{2}\left(\left(\beta_{1}, \beta_{2}\right) \times \mathscr{O}\right)}\right)^{\delta} .
$$

Next, we note that we have

$$
\begin{aligned}
& \|u\|_{H^{3}(Y)} \geq\|u\|_{L^{2}\left(\left(\beta, S_{0}-\beta\right), H^{3}(\Omega)\right)} \geq e^{-C|\sigma|^{1 / 2}}\left\|u_{0}\right\|_{H^{3}(\Omega)}, \\
& \|u\|_{H^{3}(Z)} \lesssim e^{C|\sigma|^{1 / 2}}\left\|u_{0}\right\|_{H^{3}(\Omega)}, \\
& \|u\|_{L^{2}\left(\left(\beta_{1}, \beta_{2}\right) \times \mathscr{O}\right)} \leq e^{C|\sigma|^{1 / 2}}\left\|u_{0}\right\|_{L^{2}(\mathscr{O})},
\end{aligned}
$$

yielding with (6.5)

$$
\left\|u_{0}\right\|_{H^{3}(\Omega)} \leq C e^{C|\sigma|^{1 / 2}}\left(\|f\|_{L^{2}(Z)}+\left\|u_{0}\right\|_{L^{2}(\mathscr{O})}\right) .
$$

This concludes the proof of the estimate of Lemma 6.1. 
6.3. A stabilization result for the plate equation. Let now $\left(y_{0}, y_{1}\right) \in D\left(\mathcal{B}^{k}\right), k \geq 1$, and $y$ be the solution of the damped plate equation

$$
\partial_{t}^{2} y+\Delta^{2} y+\alpha \partial_{t} y=0, \quad y_{\mid t=0}=y_{0}, \partial_{t} y_{\mid t=0}=y_{1}, \quad y_{\mid[0,+\infty) \times \partial \Omega}=\partial_{v} y_{\mid[0,+\infty) \times \partial \Omega}=0,
$$

with $\alpha$ a nonnegative function such that $y \geq \delta>0$ on $\mathscr{O}$, an open subset of $\Omega$. If we set $Y=\left(y, \partial_{t} y\right)$ we have $\left(\partial_{t}+\mathcal{B}\right) Y=0$. From the resolvent estimate of Theorem 4.17 we obtain the following energy decay for the damped plate equation, using the results set in an abstract framework in [BD08].

Theorem 6.2. With the energy function introduced in (1.16) the solution to the damped plate equation (6.6) satisfies, for some $C>0$,

$$
E(y)(t) \leq \frac{C}{(\log (2+t))^{4 k}}\left\|\mathcal{B}^{k} Y_{0}\right\|_{\mathscr{H}}^{2}, \quad t>0, \quad Y_{0}=\left(y_{0}, y_{1}\right) \in D\left(\mathcal{B}^{k}\right) .
$$

Among the existing results available in the literature for plate type equations, many of them concern the "hinged" boundary conditions. We first mention these result. An important result obtained in [Jaf90] on the controllability of the plate equation on a rectangle domain with an arbitrary small control domain. The method relies on the generalization of Ingham type inequalities in [Kah62]. An exponential stabilization result, in the same geometry, can be found in [RTT06], using similar techniques. In [RTT06] the localized damping term involves the time derivative $\partial_{t} y$ as in (6.6). Interior nonlinear feedbacks can be used for exponential stabilization [Teb09]. There, feedbacks are localized in a neighborhood of part of the boundary that fulfills multiplier-type conditions. A general analysis of nonlinear damping that includes the plate equation is provided in [ABA11] under multiplier-type conditions. For "hinged" boundary conditions also, with a boundary damping term, we cite [ATT07] where, on a square domain, a necessary and sufficient condition is provided for exponential stabilization. In [Nou09], a polynomial stabilization rate is obtained if the condition of [ATT07] is relaxed.

For "clamped" boundary conditions, few results are available. We cite [AB06], where a general analysis of nonlinearly damped systems that includes the plate equation under multiplier-type conditions is provided. In [ABPT17], the analysis of discretized general nonlinearly damped system is also carried out, with the plate equation as an application. In [Teb12], a nonlinear damping involving the p-Laplacian is used also under multiplier-type conditions. In [DS15], an exponential decay is obtained in the case of "clamped" boundary conditions, yet with a damping term of the Kelvin-Voigt type, that is of the form $\partial_{t} \Delta y$, that acts over the whole domain.

Theorem 6.2 provides a log-type stabilization result. Optimality is a natural question and one could be interested in seeking geometries that improve this decay rate, yielding polynomial or exponential rate, in the case of "clamped" boundary conditions, in the spirit of some of the existing results we cite above.

\section{APPENDICES}

\section{A. Proofs of some technical Results}

A.1. Proof of the estimate optimality in the case of symbol flatness. Here, we provide a proof of Proposition 1.6.

We have $Q\left(z, D_{z}\right)=q\left(z, D_{z}\right)+r_{m-1}\left(z, D_{z}\right)+r_{m-2}\left(z, D_{z}\right)$ with $r_{m-1}\left(z, D_{z}\right)$ homogeneous of degree $m-1$ and $r_{m-2}\left(z, D_{z}\right)$ of order $m-2$ (non necessarily homogeneous).

If there exists $\left(z_{0}, \zeta_{0}, \tau_{0}\right)$ as in the statement of the proposition, then by homogeneity, as $\tau_{0} \neq 0$, there exists $\zeta_{1} \in \mathbb{R}^{N}$, such that

$$
q\left(z_{0}, \theta_{1}\right)=0, \quad d_{z, \zeta} q\left(z_{0}, \theta_{1}\right)=0, \quad \theta_{1}^{\alpha} \neq 0, \text { with } \theta_{1}=\zeta_{1}+i d \varphi\left(z_{0}\right) .
$$


Without any loss of generality we may assume that $z_{0}=0$. Because of the form of (1.14), observe also that there is no loss of generality if we assume that $\varphi(0)=0$.

We then introduce $w(z)=\left\langle z, \theta_{1}\right\rangle$. We note that

$$
\varphi(z)-\operatorname{Im} w(z)=G(z)+|z|^{3} \mathscr{O}(1), \quad G(z)=\frac{1}{2} d_{z}^{2} \varphi(0)(z, z) .
$$

We then pick $f \in \mathscr{C}_{c}^{\infty}\left(\mathbb{R}^{N}\right), f \not \equiv 0$, and set $u_{\tau}(z)=e^{i \tau w(z)} f\left(\tau^{1 / 2} z\right)$. We have

$$
\begin{aligned}
\left\|e^{\tau \varphi} u_{\tau}\right\|_{L^{2}\left(\mathbb{R}^{N}\right)}^{2} & =\int_{\mathbb{R}^{N}} e^{2 \tau\left(G(z)+|z|^{3} \mathscr{O}(1)\right)} \mid f\left(\left.\tau^{1 / 2} z\right|^{2} d z=\tau^{-N / 2} \int_{\mathbb{R}^{N}} e^{2 G(y)+\tau^{-1 / 2}|y|^{3} \mathscr{O}(1)}|f(y)|^{2} d y\right. \\
& \underset{\tau \rightarrow \infty}{\sim} \tau^{-N / 2} \int_{\mathbb{R}^{N}} e^{2 G(y)}|f(y)|^{2} d y,
\end{aligned}
$$

with the change of variables $y=\tau^{1 / 2} z$ and the dominated convergence theorem.

As we note that

$$
e^{-i \tau w(z)} D_{z}^{\alpha} u_{\tau}=\left(D_{z}+\tau \theta_{1}\right)^{\alpha} f\left(\tau^{1 / 2} z\right)=\tau^{|\alpha|} \theta_{1}^{\alpha} f\left(\tau^{1 / 2} z\right)+\tau^{|\alpha|-1 / 2} \mathscr{O}(1),
$$

similarly, we find

$$
\left\|e^{\tau \varphi} D_{z}^{\alpha} u_{\tau}\right\|_{L^{2}\left(\mathbb{R}^{N}\right)}^{2} \underset{\tau \rightarrow \infty}{\sim} \tau^{2|\alpha|-N / 2}\left|\theta_{1}^{\alpha}\right|^{2} \int_{\mathbb{R}^{N}} e^{2 G(y)}|f(y)|^{2} d y,
$$

as we have $\theta_{1}^{\alpha} \neq 0$.

We have

$$
e^{-i \tau w(z)} Q e^{i \tau w(z)}=q\left(z, D_{z}+\tau \theta_{1}\right)+r_{m-1}\left(z, D_{z}+\tau \theta_{1}\right)+r_{m-2}\left(z, D_{z}+\tau \theta_{1}\right) .
$$

With the Taylor formula and homogeneity we observe that

$$
\begin{aligned}
q\left(z, D_{z}+\tau \theta_{1}\right)= & \tau^{m} q\left(z, \theta_{1}\right)+\tau^{m-1} d_{\zeta} q\left(z, \theta_{1}\right)\left(D_{z}\right)+\frac{1}{2} \tau^{m-2} d_{\zeta}^{2} q\left(z, \theta_{1}\right)\left(D_{z}, D_{z}\right) \\
& +\frac{1}{2} \int_{0}^{1}(1-t)^{2} d_{\zeta}^{3} q\left(z, t D_{z}+\tau \theta_{1}\right)\left(D_{z}, D_{z}, D_{z}\right) d t
\end{aligned}
$$

Next, we write

$$
\begin{gathered}
q\left(z, \theta_{1}\right)=\underbrace{q\left(0, \theta_{1}\right)}_{=0}+\underbrace{d_{z} q\left(0, \theta_{1}\right)(z)}_{=0}+\frac{1}{2} d_{z}^{2} q\left(0, \theta_{1}\right)(z, z)+\frac{1}{2} \int_{0}^{1}(1-t)^{2} d_{z}^{3} q\left(t z, \theta_{1}\right)(z, z, z) d t, \\
d_{\zeta} q\left(z, \theta_{1}\right)\left(D_{z}\right)=\underbrace{d_{\zeta} q\left(0, \theta_{1}\right)\left(D_{z}\right)}_{=0}+d_{\zeta} d_{z} q\left(0, \theta_{1}\right)\left(D_{z}, z\right)+\int_{0}^{1}(1-t) d_{\zeta} d_{z}^{2} q\left(t z, \theta_{1}\right)\left(D_{z}, z, z\right) d t,
\end{gathered}
$$

and

$$
d_{\zeta}^{2} q\left(z, \theta_{1}\right)\left(D_{z}, D_{z}\right)=d_{\zeta}^{2} q\left(0, \theta_{1}\right)\left(D_{z}, D_{z}\right)+\int_{0}^{1} d_{\zeta}^{2} d_{z} q\left(t z, \theta_{1}\right)\left(D_{z}, D_{z}, z\right) d t,
$$


which gives

$$
\begin{aligned}
e^{-i \tau w(z)} Q e^{i \tau w(z)}=\tau^{m-1}( & \frac{1}{2} d_{z}^{2} q\left(0, \theta_{1}\right)\left(\tau^{1 / 2} z, \tau^{1 / 2} z\right)+\frac{1}{2} \tau^{-1 / 2} \int_{0}^{1}(1-t)^{2} d_{z}^{3} q\left(t z, \theta_{1}\right)\left(\tau^{1 / 2} z, \tau^{1 / 2} z, \tau^{1 / 2} z\right) d t \\
& +\tau^{-1 / 2} d_{\zeta} d_{z} q\left(0, \theta_{1}\right)\left(D_{z}, \tau^{1 / 2} z\right)+\tau^{-1} \int_{0}^{1}(1-t) d_{\zeta} d_{z}^{2} q\left(t z, \theta_{1}\right)\left(D_{z}, \tau^{1 / 2} z, \tau^{1 / 2} z\right) d t \\
& +\frac{1}{2} \tau^{-1} d_{\zeta}^{2} q\left(0, \theta_{1}\right)\left(D_{z}, D_{z}\right)+\frac{1}{2} \tau^{-3 / 2} \int_{0}^{1} d_{\zeta}^{2} d_{z} q\left(t z, \theta_{1}\right)\left(D_{z}, D_{z}, \tau^{1 / 2} z\right) d t \\
& \left.+\tau^{1-m}\left(r_{m-1}\left(z, D_{z}+\tau \theta_{1}\right)+r_{m-2}\left(z, D_{z}+\tau \theta_{1}\right)\right)\right) .
\end{aligned}
$$

We then find

$$
\begin{aligned}
e^{-i \tau w(z)} Q u_{\tau}=\tau^{m-1} & \left(\frac{1}{2} d_{z}^{2} q\left(0, \theta_{1}\right)\left(\tau^{1 / 2} z, \tau^{1 / 2} z\right) f\left(\tau^{1 / 2} z\right)+d_{\zeta} d_{z} q\left(0, \theta_{1}\right)\left(D_{z} f\left(\tau^{1 / 2} z\right), \tau^{1 / 2} z\right)\right. \\
& \left.+\frac{1}{2}\left(d_{\zeta}^{2} q\left(0, \theta_{1}\right)\left(D_{z}, D_{z}\right) f\right)\left(\tau^{1 / 2} z\right)+r_{m-1}\left(z, \theta_{1}\right) f\left(\tau^{1 / 2} z\right)+\tau^{-1 / 2} \mathscr{O}(1)\right) .
\end{aligned}
$$

Arguing as for (A.2), we obtain, as $\tau \rightarrow \infty$,

$$
\begin{aligned}
\left\|e^{\tau \varphi} Q u_{\tau}\right\|_{L^{2}\left(\mathbb{R}^{N}\right)}^{2}= & \tau^{2(m-1)-N / 2} \int_{\mathbb{R}^{N}} e^{2 G(y)} \mid \frac{1}{2} d_{z}^{2} q\left(0, \theta_{1}\right)(y, y) f(y)+d_{\zeta} d_{z} q\left(0, \theta_{1}\right)\left(D_{z} f(y), y\right) \\
& +\frac{1}{2}\left(d_{\zeta}^{2} q\left(0, \theta_{1}\right)\left(D_{z}, D_{z}\right) f\right)(y)+\left.r_{m-1}\left(0, \theta_{1}\right) f(y)\right|^{2} d y \\
& +\mathscr{O}\left(\tau^{2(m-1)-N / 2-1 / 2}\right) .
\end{aligned}
$$

The assumed estimate (1.14) along with (A.2)-(A.4) thus implies that $\delta=0$ and moreover that the integral above does not vanish.

Remark A.1. Observe that if in addition we assume that $m \geq 3$ then the partial Carleman estimate (1.14) with the loss of a full derivative implies that $d \varphi(z)$ does not vanish in $\Omega$. In fact, if $d \varphi\left(z_{0}\right)=0$ and if we pick $\zeta_{0}=0$ then $\theta_{1}=0$ and since $m \geq 3$ we have the properties listed in (A.1). The remainder of the proof then yields a contradiction as the integral term in (A.4) vanishes.

In the case $m=1$, it is known that a Carleman estimate with the loss of a half derivative can hold even if the gradient of the weight function vanishes (see Lemma 8.1.1 in [Hör63]). For instance, for $\varphi(z)=z_{1}^{2} / 2$ and for the operator $D_{z_{1}}$, we have

$$
\tau^{1 / 2}\left\|e^{\tau \varphi} u\right\|_{L^{2}\left(\mathbb{R}^{N}\right)} \lesssim\left\|e^{\tau \varphi} D_{z_{1}} u\right\|_{L^{2}\left(\mathbb{R}^{N}\right)},
$$

for $\tau>0$ and $u \in \mathscr{C}_{c}^{\infty}\left(\mathbb{R}^{N}\right)$. Then, for the operator $D_{z_{1}}^{2}$, we have

$$
\tau\left\|e^{\tau \varphi} u\right\|_{L^{2}\left(\mathbb{R}^{N}\right)} \lesssim\left\|e^{\tau \varphi} D_{z_{1}}^{2} u\right\|_{L^{2}\left(\mathbb{R}^{N}\right)},
$$

for $\tau>0$ and $u \in \mathscr{C}_{c}^{\infty}\left(\mathbb{R}^{N}\right)$. We then have the case of an operator of order $m=1$ or 2 in $\mathbb{R}^{N}$ such that an estimate with a loss a full derivative holds and yet $d \varphi$ may vanish.

Remark A.2. The reader should observe that the statement of Proposition 1.6 assumes that the symbol $q(z, \zeta+i \tau d \varphi(z))$ vanishes at second order at a complex root, that is, for $\tau>0$. Flatness at a real root may not yield $\delta=0$. In fact, in $\mathbb{R}^{N}, N \geq 2$, consider the operator $Q=\left(D_{z_{1}}+D_{z_{2}}\right)^{m}$ with $m \geq 2$ and $\varphi(z)=z_{1}$. Then $q(\zeta+i \tau d \varphi)=\left(\zeta_{1}+\zeta_{2}+i \tau\right)^{m}$ which vanishes (at order $m$ ) for $\tau=0$ and $\zeta_{1}+\zeta_{2}=0$. Yet, we have the following estimate

$$
\tau^{m}\left\|e^{\tau \varphi} u\right\|_{L^{2}\left(\mathbb{R}^{N}\right)} \leq\left\|e^{\tau \varphi} P u\right\|_{L^{2}\left(\mathbb{R}^{N}\right)},
$$

for $v \in \mathscr{C}_{c}^{\infty}\left(\mathbb{R}^{2}\right)$. This means $\delta=1$ here. 
The proof of (A.5) is as follows. We write $e^{\tau \varphi}\left(D_{z_{1}}+D_{z_{2}}\right) u=\left(D_{z_{1}}+i \tau+D_{z_{2}}\right) v$ with $v=e^{\tau \varphi} u$. We then have

$$
\begin{aligned}
\left\|e^{\tau \varphi}\left(D_{z_{1}}+D_{z_{2}}\right) u\right\|_{L^{2}\left(\mathbb{R}^{N}\right)}^{2} & =\left\|\left(D_{z_{1}}+D_{z_{2}}\right) v\right\|_{L^{2}\left(\mathbb{R}^{N}\right)}^{2}+\|\tau v\|_{L^{2}\left(\mathbb{R}^{N}\right)}^{2}-2 i \tau \operatorname{Re} \int \bar{v}\left(D_{z_{1}}+D_{z_{2}}\right) v d z \\
& =\left\|\left(D_{z_{1}}+D_{z_{2}}\right) v\right\|_{L^{2}\left(\mathbb{R}^{N}\right)}^{2}+\|\tau v\|_{L^{2}\left(\mathbb{R}^{N}\right)}^{2}-\tau \underbrace{\int\left(\partial_{z_{1}}+\partial_{z_{2}}\right)|v|^{2} d z}_{=0 \text { as supp }(v) \operatorname{compact}} \\
& \geq \tau^{2}\|v\|_{L^{2}\left(\mathbb{R}^{N}\right)}^{2}=\tau^{2}\left\|e^{\tau \varphi} u\right\|_{L^{2}\left(\mathbb{R}^{N}\right)}^{2} .
\end{aligned}
$$

Multiple applications of this estimate yield (A.5).

Note however that we do not claim to have $\left\|e^{\tau \varphi} D u\right\|_{L^{2}\left(\mathbb{R}^{N}\right)} \lesssim\left\|e^{\tau \varphi}\left(D_{z_{1}}+D_{z_{2}}\right) u\right\|_{L^{2}\left(\mathbb{R}^{N}\right)}$, as $D_{z_{1}}+D_{z_{2}}$ is not elliptic.

\section{A.2. Proofs associated with the semi-classical calculus.}

A.2.1. Proof of Proposition 4.2. The dual quadratic form of $g$ on $\mathcal{W}$ is given by

$$
g^{\sigma}=\lambda_{\tilde{\tau}}^{2}|d z|^{2}+\frac{\left|d \zeta^{\prime}\right|^{2}}{(1+\gamma \varepsilon)^{2}}+\frac{\left|d \zeta_{N}\right|^{2}}{\gamma^{2}}
$$

We then have, for $X=\left(z_{X}, \zeta_{X}\right)$, as $\gamma \geq 1$,

$$
\begin{aligned}
\left(h_{g}\right)^{-1}(X) & =\inf _{\substack{T \in \mathcal{W} \\
T \neq 0}}\left(g_{X}^{\sigma}(T) / g_{X}(T)\right)^{1 / 2}=\min \left(\gamma^{-1},(1+\gamma \varepsilon)^{-1}\right) \lambda_{\tilde{\tau}}(X) \\
& \geq(2 \gamma)^{-1} \lambda_{\tilde{\tau}}(X) \geq \tau \varphi\left(z_{X}\right) / 2 \geq 1,
\end{aligned}
$$

as $\tau \geq \tau_{*} \geq 2$. The uncertainty principle is thus fulfilled.

For $X=\left(z_{X}, \zeta_{X}\right) \in \mathcal{W}$, we write $z_{X}=\left(z_{X}^{\prime},\left(z_{X}\right)_{N}\right)$, with $z_{X}^{\prime} \in \mathbb{R}^{N-1}$. Similarly, we also write $\zeta_{X}=\left(\zeta_{X}^{\prime},\left(\zeta_{X}\right)_{N}\right)$, with $\zeta_{X}^{\prime} \in \mathbb{R}^{N-1}$.

We now prove the slow variations of $g$ and $\varphi, \lambda_{\tilde{\tau}}$, namely, there exist $K>0, r>0$, such that

$$
\forall X, Y, T \in \mathcal{W}, \quad g_{X}(Y-X) \leq r^{2} \Rightarrow\left\{\begin{array}{l}
g_{Y}(T) \leq K g_{X}(T), \\
K^{-1} \leq \frac{\varphi\left(z_{X}\right)}{\varphi\left(z_{Y}\right)} \leq K, \quad K^{-1} \leq \frac{\lambda_{\tilde{\tau}}(X)}{\lambda_{\tilde{\tau}}(Y)} \leq K,
\end{array}\right.
$$

where $X=\left(z_{X}, \zeta_{X}\right)$ and $Y=\left(z_{Y}, \zeta_{Y}\right)$. We thus assume that $g_{X}(Y-X) \leq r^{2}$, with $0<r<1$ to be chosen below. This gives

$$
(1+\gamma \varepsilon)\left(\left|z_{X}^{\prime}-z_{Y}^{\prime}\right|\right)+\gamma\left|\left(z_{X}\right)_{N}-\left(z_{Y}\right)_{N}\right|+\lambda_{\tilde{\tau}}(X)^{-1}\left(\left|\zeta_{X}-\zeta_{Y}\right|\right) \leq C r .
$$

We observe that we have

$$
\varphi\left(z_{X}\right)=e^{\gamma \psi_{\varepsilon}\left(z_{X}\right)}=\varphi\left(z_{Y}\right) e^{\gamma\left(\psi_{\varepsilon}\left(z_{X}\right)-\psi_{\varepsilon}\left(z_{Y}\right)\right)},
$$

where $\psi_{\varepsilon}\left(z_{X}\right)=\psi\left(\varepsilon z_{X}^{\prime},\left(z_{X}\right)_{N}\right)$. Note that

$$
\left|\psi_{\varepsilon}\left(z_{X}\right)-\psi_{\varepsilon}\left(z_{Y}\right)\right| \leq\left(\varepsilon\left|z_{X}^{\prime}-z_{Y}^{\prime}\right|+\left|\left(z_{X}\right)_{N}-\left(z_{Y}\right)_{N}\right|\right)|| \psi^{\prime} \|_{L^{\infty}} .
$$

With (A.6), we thus obtain

$$
\varphi\left(z_{X}\right) \leq \varphi\left(z_{Y}\right) e^{C r\left\|\psi^{\prime}\right\|_{L^{\infty}}} \lesssim \varphi\left(z_{Y}\right)
$$

Similarly, we have

$$
\varphi\left(z_{Y}\right) \lesssim \varphi\left(z_{X}\right)
$$

We also have

$$
\left|\zeta_{Y}\right| \leq\left|\zeta_{Y}-\zeta_{X}\right|+\left|\zeta_{X}\right| \leq C r \lambda_{\tilde{\tau}}(X)+\left|\zeta_{X}\right| \lesssim \lambda_{\tilde{\tau}}(X) .
$$


Next, we write

$$
\left|\zeta_{X}\right| \leq\left|\zeta_{Y}-\zeta_{X}\right|+\left|\zeta_{Y}\right| \leq C r \lambda_{\tilde{\tau}}(X)+\left|\zeta_{Y}\right| \leq C r\left(\tau \gamma \varphi\left(z_{X}\right)+\left|\zeta_{X}\right|\right)+\left|\zeta_{Y}\right| .
$$

Hence, for $r$ sufficiently small, with (A.7), we have

$$
\left|\zeta_{X}\right| \lesssim \tau \gamma \varphi\left(z_{X}\right)+\left|\zeta_{Y}\right| \lesssim \lambda_{\tilde{\tau}}(Y)
$$

With (A.7) and (A.10), resp. (A.8) and (A.9), we find

$$
\lambda_{\tilde{\tau}}(X) \lesssim \lambda_{\tilde{\tau}}(Y), \quad \operatorname{resp} . \quad \lambda_{\tilde{\tau}}(Y) \lesssim \lambda_{\tilde{\tau}}(X) .
$$

Then, if $T=\left(z_{T}, \zeta_{T}\right) \in \mathcal{W}$ we find

$$
\frac{\left|\zeta_{T}\right|^{2}}{\lambda_{\tilde{\tau}}(Y)^{2}} \lesssim \frac{\left|\zeta_{T}\right|^{2}}{\lambda_{\tilde{\tau}}(X)^{2}} \lesssim \frac{\left|\zeta_{T}\right|^{2}}{\lambda_{\tilde{\tau}}(Y)^{2}},
$$

and this gives $g_{Y}(T) \lesssim g_{X}(T) \lesssim g_{Y}(T)$, concluding the proof of the slow variations of $\lambda_{\tilde{\tau}}$ and $g$.

We now prove the temperance of $g, \varphi$ and $\lambda_{\tilde{\tau}}$, namely, there exist $K>0, N>0$, such that

$$
\begin{aligned}
& \forall X, Y, T \in \mathcal{W}, \quad \frac{g_{X}(T)}{g_{Y}(T)} \leq C\left(1+g_{X}^{\sigma}(X-Y)\right)^{N}, \\
& \forall X, Y \in \mathcal{W}, \quad \frac{\varphi\left(z_{X}\right)}{\varphi\left(z_{Y}\right)} \leq C\left(1+g_{X}^{\sigma}(X-Y)\right)^{N}, \quad \frac{\lambda_{\tilde{\tau}}(X)}{\lambda_{\tilde{\tau}}(Y)} \leq C\left(1+g_{X}^{\sigma}(X-Y)\right)^{N},
\end{aligned}
$$

where $X=\left(z_{X}, \zeta_{X}\right)$ and $Y=\left(z_{Y}, \zeta_{Y}\right)$. We have

$$
g_{X}^{\sigma}(X-Y)=\lambda_{\tilde{\tau}}(X)^{2}\left|z_{X}-z_{Y}\right|^{2}+\frac{\left|\zeta_{X}^{\prime}-\zeta_{Y}^{\prime}\right|^{2}}{(1+\gamma \varepsilon)^{2}}+\frac{\left|\left(\zeta_{X}\right)_{N}-\left(\zeta_{Y}\right)_{N}\right|^{2}}{\gamma^{2}} .
$$

We note that

$$
\begin{aligned}
\left|\zeta_{X}\right| & \leq\left|\zeta_{Y}\right|+\left|\zeta_{X}-\zeta_{Y}\right| \leq\left|\zeta_{Y}\right|+\frac{\left|\zeta_{X}-\zeta_{Y}\right|}{\gamma} \tau \gamma \varphi\left(z_{Y}\right) \\
& \leq\left|\zeta_{Y}\right|+\left(2 \frac{\left|\zeta_{X}^{\prime}-\zeta_{Y}^{\prime}\right|}{1+\gamma \varepsilon}+\frac{\left|\left(\zeta_{X}\right)_{N}-\left(\zeta_{Y}\right)_{N}\right|}{\gamma}\right) \tau \gamma \varphi\left(z_{Y}\right) \\
& \lesssim\left(1+g_{X}^{\sigma}(X-Y)^{1 / 2}\right) \lambda_{\tilde{\tau}}(Y) .
\end{aligned}
$$

First, if $(1+\varepsilon \gamma)\left|z_{X}^{\prime}-z_{Y}^{\prime}\right|+\gamma\left|\left(z_{X}\right)_{N}-\left(z_{Y}\right)_{N}\right| \leq 1$, then, arguing as in (A.7), we find

$$
\varphi\left(z_{X}\right) \lesssim \varphi\left(z_{Y}\right), \quad \tau \gamma \varphi\left(z_{X}\right) \lesssim \lambda_{\tilde{\tau}}(Y) .
$$

Second, if $(1+\varepsilon \gamma)\left|z_{X}^{\prime}-z_{Y}^{\prime}\right|+\gamma\left|\left(z_{X}\right)_{N}-\left(z_{Y}\right)_{N}\right| \geq 1$, we then have $2\left|z_{X}-z_{Y}\right| \geq 1 / \gamma$. We write, as $\tau \geq \tau_{*} \geq 1$,

$$
\varphi\left(z_{X}\right)=\frac{\tilde{\tau}\left(z_{X}\right)}{\gamma \tau} \leq \frac{\lambda_{\tilde{\tau}}(X)}{\gamma} \lesssim\left|z_{X}-z_{Y}\right| \lambda_{\tilde{\tau}}(X) \lesssim\left(1+g_{X}^{\sigma}(X-Y)^{1 / 2}\right) \lesssim\left(1+g_{X}^{\sigma}(X-Y)^{1 / 2} \varphi\left(z_{Y}\right),\right.
$$

using that $\varphi \geq 1$. We also write

$$
\tau \gamma \varphi\left(z_{X}\right) \lesssim \lambda_{\tilde{\tau}}(X) \leq \lambda_{\tilde{\tau}}(X) \frac{\lambda_{\tilde{\tau}}(Y)}{\gamma} \lesssim\left|z_{X}-z_{Y}\right| \lambda_{\tilde{\tau}}(X) \lambda_{\tilde{\tau}}(Y) \lesssim\left(1+g_{X}^{\sigma}(X-Y)^{1 / 2}\right) \lambda_{\tilde{\tau}}(Y) .
$$

In any case, we have

$$
\varphi\left(z_{X}\right) \leq\left(1+g_{X}^{\sigma}(X-Y)^{1 / 2}\right) \varphi\left(z_{Y}\right) \lesssim\left(1+g_{X}^{\sigma}(X-Y)\right) \varphi\left(z_{Y}\right),
$$

that is, the temperance of $\varphi$ and we have $\tau \gamma \varphi\left(z_{X}\right) \lesssim\left(1+g_{X}^{\sigma}(X-Y)^{1 / 2}\right) \lambda_{\tilde{\tau}}(Y)$, which, along with (A.11), yields the temperance of $\lambda_{\tilde{\tau}}$ :

$$
\lambda_{\tilde{\tau}}(X) \lesssim\left(1+g_{X}^{\sigma}(X-Y)^{1 / 2}\right) \lambda_{\tilde{\tau}}(Y) \lesssim\left(1+g_{X}^{\sigma}(X-Y)\right) \lambda_{\tilde{\tau}}(Y) .
$$


For the temperance of $g$ we need to prove

$$
(1+\varepsilon \gamma)\left|z_{T}^{\prime}\right|+\gamma\left|\left(z_{T}\right)_{N}\right|+\frac{\left|\zeta_{T}\right|}{\lambda_{\tilde{\tau}}(X)} \lesssim\left(1+g_{X}^{\sigma}(X-Y)\right)^{N}\left((1+\varepsilon \gamma)\left|z_{T}^{\prime}\right|+\gamma\left|\left(z_{T}\right)_{N}\right|+\frac{\left|\zeta_{T}\right|}{\lambda_{\tilde{\tau}}(Y)}\right),
$$

for $T=\left(z_{T}, \zeta_{T}\right) \in \mathcal{W}$. To conclude it suffices to prove

$$
\lambda_{\tilde{\tau}}(Y) \lesssim\left(1+g_{X}^{\sigma}(X-Y)\right)^{N} \lambda_{\tilde{\tau}}(X) .
$$

We have

$$
\begin{aligned}
\left|\zeta_{Y}\right| \leq\left|\zeta_{X}\right|+\left|\zeta_{X}-\zeta_{Y}\right| & \leq\left|\zeta_{X}\right|+\frac{\left|\zeta_{X}-\zeta_{Y}\right|}{\gamma} \tau \gamma \varphi\left(z_{X}\right) \\
& \leq\left|\zeta_{X}\right|+\left(2 \frac{\left|\zeta_{X}^{\prime}-\zeta_{Y}^{\prime}\right|}{1+\gamma \varepsilon}+\frac{\left|\left(\zeta_{X}\right)_{N}-\left(\zeta_{Y}\right)_{N}\right|}{\gamma}\right) \tau \gamma \varphi\left(z_{X}\right) \\
& \lesssim\left(1+g_{X}^{\sigma}(X-Y)^{1 / 2}\right) \lambda_{\tilde{\tau}}(X)
\end{aligned}
$$

It thus remains to prove

$$
\tau \gamma \varphi\left(z_{Y}\right) \lesssim\left(1+g_{X}^{\sigma}(X-Y)\right)^{N} \lambda_{\tilde{\tau}}(X) .
$$

First, if $(1+\gamma \varepsilon)\left|z_{X}^{\prime}-z_{Y}^{\prime}\right|+\gamma\left|\left(z_{X}\right)_{N}-\left(z_{Y}\right)_{N}\right| \leq 1$, then $\varphi\left(z_{Y}\right) \lesssim \varphi\left(z_{X}\right)$, arguing as in (A.8). Estimate (A.13) is then clear. Second, if $(1+\gamma \varepsilon)\left|z_{X}^{\prime}-z_{Y}^{\prime}\right|+\gamma\left|\left(z_{X}\right)_{N}-\left(z_{Y}\right)_{N}\right| \geq 1$, which implies $2\left|z_{X}-z_{Y}\right| \geq 1 / \gamma$, with (4.3) we write

$$
\begin{aligned}
\tau \gamma \varphi\left(z_{Y}\right) & \leq \tau \gamma \varphi\left(z_{X}\right)^{k+1} \lesssim \frac{\lambda_{\tilde{\tau}}(X)^{k+1}}{(\tau \gamma)^{k}} \lesssim\left(\frac{\lambda_{\tilde{\tau}}(X)}{\tau \gamma}\right)^{k} \lambda_{\tilde{\tau}}(X) \lesssim\left(\left|z_{X}-z_{Y}\right| \frac{\lambda_{\tilde{\tau}}(X)}{\tau}\right)^{k} \lambda_{\tilde{\tau}}(X) \\
& \lesssim\left(1+g_{X}^{\sigma}(X-Y)^{1 / 2}\right)^{k} \lambda_{\tilde{\tau}}(X),
\end{aligned}
$$

since $\tau \geq \tau_{*} \geq 1$. In any case, we thus have

$$
\tau \gamma \varphi\left(z_{Y}\right) \lesssim\left(1+g_{X}^{\sigma}(X-Y)^{1 / 2}\right)^{k} \lambda_{\tilde{\tau}}(X),
$$

which concludes the proof.

A.2.2. Proof of Lemma 4.4. We have $\tilde{\tau} \lesssim \lambda_{\tilde{\tau}}$ (resp. $\tilde{\tau} \lesssim \lambda_{\mathrm{T}, \tilde{\tau}}$ ) and $d_{\zeta} \tilde{\tau}=0$. Only differentiations of $\tilde{\tau}$ with respect to $z$ thus need to be considered. Recalling that $\tilde{\tau}=\tau \gamma \varphi_{\gamma, \varepsilon}$ we find that, for $\alpha=\left(\alpha^{\prime}, \alpha_{N}\right) \in \mathbb{N}^{N}$, we can write $\partial_{z}^{\alpha} \tilde{\tau}\left(\varrho^{\prime}\right)$ as a linear combination of terms of the form

$$
\tau \gamma^{1+k} \varphi_{\gamma, \varepsilon}(z) \prod_{j=1}^{k} \partial_{z}^{\alpha^{(j)}} \psi_{\varepsilon}(z)=\tau \gamma^{1+k} \varepsilon^{\left|\alpha^{\prime}\right|} \varphi_{\gamma, \varepsilon}(z) \prod_{j=1}^{k} \partial_{z}^{\alpha^{(j)}} \psi\left(\varepsilon z^{\prime}, z_{N}\right)
$$

with $\alpha^{(1)}+\cdots+\alpha^{(k)}=\alpha,\left|\alpha^{(j)}\right| \geq 1, j=1, \ldots, k$, and $k \leq|\alpha|$, implying, as $\gamma \geq 1,\left|\partial_{z}^{\alpha} \tilde{\tau}\left(\varrho^{\prime}\right)\right| \lesssim$ $\tilde{\tau}\left(\varrho^{\prime}\right) \gamma^{|\alpha|} \varepsilon^{\left|\alpha^{\prime}\right|} \lesssim \tilde{\tau}\left(\varrho^{\prime}\right) \gamma^{\alpha_{N}}(\varepsilon \gamma)^{\left|\alpha^{\prime}\right|}$, as $\left\|\psi^{(\ell)}\right\|_{L^{\infty}} \leq C$ for any $\ell \in \mathbb{N}$, which yields the results.

A.2.3. Proof of Lemma 4.8. For $\alpha=\left(\alpha^{\prime}, \alpha_{N}\right) \in \mathbb{N}^{N}$ and $\beta^{\prime} \in \mathbb{N}^{N-1}$, we may write $\partial_{z}^{\alpha} \partial_{\zeta^{\prime}}^{\beta^{\prime}} a\left(\varrho^{\prime}\right)$ as a linear combination of terms of the form,

$$
b\left(\varrho^{\prime}\right)=\left(\prod_{j=1}^{k} \partial_{z}^{\alpha^{(j)}} \hat{\tau}_{p_{j}}\left(\varrho^{\prime}\right)\right) \partial_{z}^{\alpha^{(a)}} \partial_{\zeta^{\prime}}^{\beta^{\prime}} \partial_{\hat{t}}^{\alpha^{(b)}} \hat{a}\left(\kappa\left(\varrho^{\prime}\right)\right)
$$


for some $\alpha^{(b)} \in \mathbb{N}^{N}$, with $k=\left|\alpha^{(b)}\right|$, with $\alpha=\alpha^{(a)}+\alpha^{(1)}+\cdots+\alpha^{(k)},\left|\alpha^{(j)}\right| \geq 1$, and where $p_{j} \in\{1, \ldots, N\}, j=1, \ldots, k$. Using Lemma 4.5 and Definition 4.1, and we obtain

$$
\begin{aligned}
\left|b\left(\varrho^{\prime}\right)\right| & \lesssim \prod_{j=1}^{k}\left(\lambda_{\mathrm{T}, \tilde{\tau}}(1+\varepsilon \gamma)^{\left|\alpha^{(j) \prime}\right|} \gamma^{\left|\alpha_{N}^{(j)}\right|}\right)\left(\left|\hat{\tau}\left(\varrho^{\prime}\right)\right|+\left|\zeta^{\prime}\right|\right)^{m-\left|\beta^{\prime}\right|-\left|\alpha^{(b)}\right|} \\
& \lesssim(1+\varepsilon \gamma)^{\left|\alpha^{(1) \prime}\right|+\cdots+\left|\alpha^{(k) \prime}\right|} \gamma^{\alpha_{N}^{(1)}+\cdots+\alpha_{N}^{(k)}} \lambda_{\mathrm{T}, \tilde{\tau}}^{k}\left(\left|\hat{\tau}\left(\varrho^{\prime}\right)\right|+\left|\zeta^{\prime}\right|\right)^{m-\left|\beta^{\prime}\right|-\left|\alpha^{(b)}\right|} \\
& \lesssim(1+\varepsilon \gamma)^{\left|\alpha^{\prime}\right|} \gamma^{\alpha_{N}} \lambda_{\mathrm{T}, \tilde{\tau}}^{k}\left(\left|\hat{\tau}\left(\varrho^{\prime}\right)\right|+\left|\zeta^{\prime}\right|\right)^{m-k-\left|\beta^{\prime}\right|},
\end{aligned}
$$

as $\gamma \geq 1$. If $\hat{a}$ is polynomial then the term $b\left(\varrho^{\prime}\right)$ vanishes if $m-\left|\beta^{\prime}\right|-\left|\alpha^{(b)}\right|<0$. Thus if $m-\left|\beta^{\prime}\right|-$ $\left|\alpha^{(b)}\right| \geq 0$ and, as $|\hat{\tau}| \lesssim \tilde{\tau}$ in $\mathscr{U}$, we obtain

$$
\left|b\left(\varrho^{\prime}\right)\right| \leq(1+\varepsilon \gamma)^{\left|\alpha^{\prime}\right|} \gamma^{\alpha_{N}} \lambda_{\mathrm{T}, \tilde{\tau}}^{m-\left|\beta^{\prime}\right|},
$$

which yields the result. If $\hat{a}$ is not polynomial and if we have $\tilde{\tau} \asymp|\hat{\tau}|$, we obtain the same estimation, even if $m-\left|\beta^{\prime}\right|-\left|\alpha^{(b)}\right|<0$.

\section{A.2.4. Proof of Lemma 4.11. By applying (4.14), we have}

$$
\left\|\Lambda_{\mathrm{T}, \tilde{\tau}}^{m} \tilde{\tau}^{r} u\right\|_{+} \lesssim\left\|\mathrm{Op}_{\mathrm{T}}\left(\tilde{\tau}^{r} \lambda_{\mathrm{T}, \tilde{\tau}}^{m}\right) u\right\|_{+} .
$$

Next, we write $\mathrm{Op}_{\mathrm{T}}\left(\tilde{\tau}^{r} \lambda_{\mathrm{T}, \tilde{\tau}}^{m}\right)=\mathrm{Op}\left(\lambda_{\mathrm{T}, \tilde{\tau}}^{m}\right) \tilde{\tau}^{r}+\gamma R$, with $R \in \Psi\left(\tilde{\tau}^{r} \lambda_{\mathrm{T}, \tilde{\tau}}^{m-1}, g_{\mathrm{T}}\right)$ by the tangential calculus we have introduced. This yields, as $\tilde{\tau}^{r} \in S\left(\lambda_{\mathrm{T}, \tilde{\tau}}^{r}, g_{\mathrm{T}}\right)$,

$$
\left\|\mathrm{Op}_{\mathrm{T}}\left(\tilde{\tau}^{r} \lambda_{\mathrm{T}, \tilde{\tau}}^{m}\right) u\right\|_{+} \lesssim\left\|\mathrm{Op}\left(\lambda_{\mathrm{T}, \tilde{\tau}}^{m}\right) \tilde{\tau}^{r} u\right\|_{+}+\gamma\left\|\mathrm{Op}_{\mathrm{T}}\left(\tilde{\tau}^{r} \lambda_{\mathrm{T}, \tilde{\tau}}^{m-1}\right) u\right\|_{+},
$$

which yields (4.15) by choosing $\tau$ sufficiently large. Estimation (4.16) follows the same.

A.2.5. Proof of Lemma 4.14. By definition of the Sobolev norms introduced in Section 4.1.6 we have

$$
\left\|\tilde{\tau}^{r} w\right\|_{m, m^{\prime}, \tilde{\tau}} \asymp \sum_{j=0}^{m}\left\|D_{x_{d}}^{j}\left(\tilde{\tau}^{r} w\right)\right\|_{0, m+m^{\prime}-j, \tilde{\tau}}=\sum_{j=0}^{m}\left\|\Lambda_{\mathrm{T}, \tilde{\tau}}^{m+m^{\prime}-j} D_{x_{d}}^{j}\left(\tilde{\tau}^{r} w\right)\right\|_{+} .
$$

Let $m_{j}^{\prime \prime} \in \mathbb{R}$. We have $\left[\Lambda_{\mathrm{T}, \tilde{\tau}}^{m_{j}^{\prime \prime}}, D_{x_{d}}^{j}\right] \in \sum_{i=1}^{j} \gamma^{i} \Psi\left(\lambda_{\mathrm{T}, \tilde{\tau}}^{m_{j}^{\prime \prime}}, g_{\mathrm{T}}\right) D_{x_{d}}^{j-i}$, from the tangential calculus we have introduced. With Lemma 4.4 we have $\left[\tilde{\tau}^{r}, \Lambda_{\mathrm{T}, \tilde{\tau}}^{m_{j}^{\prime \prime}}\right] \in(1+\varepsilon \gamma) \Psi\left(\tilde{\tau}^{r} \Lambda_{\mathrm{T}, \tilde{\tau}}^{m_{j}^{\prime \prime}-1}, g_{\mathrm{T}}\right)$. With the same lemma, for $r_{j}^{\prime} \in \mathbb{R}$ we also have $\left[\tilde{\tau}^{r_{j}^{\prime}}, D_{x_{d}}^{j}\right] \in \sum_{i=1}^{j} \gamma^{i} \Psi\left(\tilde{\tau}_{j}^{r_{j}^{\prime}}, g_{\mathrm{T}}\right) D_{x_{d}}^{j-i}$. For $r=r_{j}^{\prime}+r_{j}^{\prime \prime}$, and $m+m^{\prime}-j=m_{j}^{\prime \prime}+m_{j}^{\prime \prime \prime}$, with $r_{j}^{\prime}, r_{j}^{\prime \prime} \in \mathbb{R}$ and $m_{j}^{\prime \prime}, m_{j}^{\prime \prime \prime} \in \mathbb{R}$, we thus obtain, by Proposition 4.13,

$$
\begin{gathered}
\left\|\tilde{\tau}^{r} w\right\|_{m, m^{\prime}, \tilde{\tau}} \geq \sum_{j=0}^{m}\left\|\tilde{\tau}_{j}^{r_{j}^{\prime}} \Lambda_{\mathrm{T}, \tilde{\tau}}^{m_{j}^{\prime \prime}} D_{x_{d}}^{j}\left(\tilde{\tau}^{r_{j}^{\prime \prime}} \Lambda_{\mathrm{T}, \tilde{\tau}}^{m_{j}^{\prime \prime \prime}} w\right)\right\|_{+}-C^{\prime} \sum_{j=1}^{m} \sum_{i=1}^{j} \gamma^{i}\left\|\tilde{\tau}^{r} D_{x_{d}}^{j-i} w\right\|_{0, m+m^{\prime}-j, \tilde{\tau}} \\
-C^{\prime \prime} \sum_{j=0}^{m} \gamma\left\|\tilde{\tau}^{r} D_{x_{d}}^{j} w\right\|_{0, m+m^{\prime}-j-1, \tilde{\tau}} \\
\geq \sum_{j=0}^{m}\left\|\tilde{\tau}_{j}^{r_{j}^{\prime}} \Lambda_{\mathrm{T}, \tilde{\tau}}^{m_{j}^{\prime \prime}} D_{x_{d}}^{j}\left(\tilde{\tau}^{r_{j}^{\prime \prime}} \Lambda_{\mathrm{T}, \tilde{\tau}}^{m_{j}^{\prime \prime \prime}} w\right)\right\|_{+}-C^{\prime} \sum_{j=0}^{m-1} \sum_{i=1}^{m} \gamma^{i}\left\|\tilde{\tau}^{r} D_{x_{d}}^{j} w\right\|_{0, m+m^{\prime}-j-i, \tilde{\tau}} \\
\quad-C^{\prime \prime} \sum_{j=0}^{m} \gamma\left\|\tilde{\tau}^{r} D_{x_{d}}^{j} w\right\|_{0, m+m^{\prime}-j-1, \tilde{\tau}} .
\end{gathered}
$$

With the argument given in (4.17), we have

$$
\sum_{j=0}^{m-1} \sum_{i=1}^{m} \gamma^{i}\left\|\tilde{\tau}^{r} D_{x_{d}}^{j} w\right\|_{0, m+m^{\prime}-j-i, \tilde{\tau}}+\sum_{j=0}^{m} \gamma\left\|\tilde{\tau}^{r} D_{x_{d}}^{j} w\right\|_{0, m+m^{\prime}-j-1, \tilde{\tau}} \ll\left\|\tilde{\tau}^{r} w\right\|_{m, m^{\prime}, \tilde{\tau}}
$$


for $\tau$ chosen sufficiently large, and we thus find

$$
\left\|\tilde{\tau}^{r} w\right\|_{m, m^{\prime}, \tilde{\tau}} \gtrsim \sum_{j=0}^{m}\left\|\tilde{\tau}^{r_{j}^{\prime}} \Lambda_{\mathrm{T}, \tilde{\tau}}^{m_{j}^{\prime \prime}} D_{x_{d}}^{j}\left(\tilde{\tau}_{j}^{r_{j}^{\prime \prime}} \Lambda_{\mathrm{T}, \tilde{\tau}}^{m_{j}^{\prime \prime \prime}} w\right)\right\|_{+},
$$

for $\tau$ chosen sufficiently large. Similarly, we find that

$$
\left\|\tilde{\tau}^{r} w\right\|_{m, m^{\prime}, \tilde{\tau}} \lesssim \sum_{j=0}^{m}\left\|\tilde{\tau}_{j}^{r^{\prime}} \Lambda_{\mathrm{T}, \tilde{\tau}}^{m_{j}^{\prime \prime}} D_{x_{d}}^{j}\left(\tilde{\tau}_{j}^{r_{j}^{\prime \prime}} \Lambda_{\mathrm{T}, \tilde{\tau}}^{m_{j}^{\prime \prime \prime}} w\right)\right\|_{+},
$$

for $\tau$ chosen sufficiently large. The result for the trace norms is obtained arguing the same.

B. Elliptic and sub-elliptic estimates at the boundary $\left(0, S_{0}\right) \times \partial \Omega$

B.1. Roots with negative imaginary part: a perfect elliptic estimate. For $z_{0} \in \partial Z, V$ denotes the neighborhood introduced in Section 4.2. We recall that $\mathcal{M}_{\mathrm{T}, V}=V \times \mathbb{R}^{N-1} \times\left[\tau_{*},+\infty\right) \times[1,+\infty) \times$ $[0,1]$.

Let $\ell(\varrho) \in S_{\tilde{\tau}}^{m, 0}$, with $\varrho=(z, \zeta, \tau, \gamma, \varepsilon)$ and $m \geq 1$, be polynomial in $\zeta_{N}$ with homogeneous coefficients in $\left(\zeta^{\prime}, \hat{\tau}\right)$ and $L=\ell\left(z, D_{z}, \tau, \gamma, \varepsilon\right)$.

Lemma B.1. Let $\mathscr{U}$ be a conic open subset of $\mathcal{M}_{\mathrm{T}, V}$. We assume that, for $\ell\left(\varrho^{\prime}, \zeta_{N}\right)$ viewed as a polynomial in $\zeta_{N}$, for $\varrho^{\prime} \in \mathscr{U}$,

- the leading coefficient is 1 ;

- all roots of $\ell\left(\varrho^{\prime}, \zeta_{N}\right)=0$ have negative imaginary part.

Let $\chi\left(\varrho^{\prime}\right) \in S\left(1, g_{T}\right)$, be homogeneous of degree zero and such that $\operatorname{supp}(\chi) \subset \mathscr{U}$. Then, for any $M \in \mathbb{N}$, there exist $C>0, \tau_{0} \geq \tau_{*}, \gamma_{0} \geq 1$ such that

$$
\left\|\mathrm{Op}_{\mathrm{T}}(\chi) w\right\|_{m, 0, \tilde{\tau}}+\left|\operatorname{tr}\left(\mathrm{Op}_{\mathrm{T}}(\chi) w\right)\right|_{m-1,1 / 2, \tilde{\tau}} \leq C\left(\left\|L \mathrm{Op}_{\mathrm{T}}(\chi) w\right\|_{+}+\|w\|_{m,-M, \tilde{\tau}}\right),
$$

for $w \in \mathscr{S}\left(\overline{\mathbb{R}_{+}^{N}}\right)$ and $\tau \geq \tau_{0}, \gamma \geq \gamma_{0}, \varepsilon \in[0,1]$.

This lemma can be proven by adapting the proof of [BL15, Lemma 6.5] to the semi-classical calculus we use here. For the notion of homogeneity for symbols and conic sets in the present calculus, we refer to Section 4.1.5.

B.2. Sub-ellipticity quantification. For $z_{0} \in \partial Z, V$ denotes the neighborhood introduced in Section 4.2. We let the function $\psi$ be as introduced in Section 4, satisfying (4.2) and (4.20), and we recall that $\psi_{\varepsilon}(z)=\psi\left(\varepsilon z^{\prime}, z_{N}\right)$ and $\varphi(z)=\exp \left(\gamma \psi_{\varepsilon}(z)\right)$. We also recall that $\lambda_{\tilde{\tau}}^{2}=\tilde{\tau}^{2}+|\zeta|^{2}$ with $\tilde{\tau}\left(\varrho^{\prime}\right)=\tau \gamma \varphi(z)$.

Proposition B.2. Let $\ell(z, \zeta)$ be polynomial of degree $m$ in $\zeta$, with smooth coefficient in $z$. We assume that for any $M \in \mathbb{R}^{N} \backslash\{0\}$, the symbol $\ell$ satisfies the simple-characteristic property in direction $M$ in a neighborhood of $V$ (see Definition 2.1). There exist $C>0$ and $\gamma_{0} \geq 1$ such that,

$$
\left|\ell\left(z, \zeta+i \hat{\tau}\left(\varrho^{\prime}\right)\right)\right|^{2}+\tau \varphi(z)\left|\psi_{\varepsilon}^{\prime}(z)\right|^{2}\left\{\operatorname{Re} \ell\left(z, \zeta+i \hat{\tau}\left(\varrho^{\prime}\right)\right), \operatorname{Im} \ell\left(z, \zeta+i \hat{\tau}\left(\varrho^{\prime}\right)\right)\right\} \geq C \lambda_{\tilde{\tau}}^{2 m},
$$

for $z \in \bar{V}, \zeta \in \mathbb{R}^{N}, \tau \geq \tau_{*}, \gamma \geq \gamma_{0}$ and $\varepsilon \in[0,1]$.

Proof. We have $0<C_{0} \leq\left|\psi_{\varepsilon}^{\prime}(z)\right| \leq C_{1}$ for $z \in \bar{V}$ and we set $K=\left\{M \in \mathbb{R}^{N} ; C_{0} \leq|M| \leq C_{1}\right\}$. As $V$ is assumed bounded (see section 4.2), we consider the compact set

$$
\mathscr{C}=\left\{(z, \zeta, \theta, M) ; \theta^{2}+|\zeta|^{2}=1, \quad z \in \bar{V}, \zeta \in \mathbb{R}^{N}, \theta \in \mathbb{R}_{+}, M \in K\right\} .
$$

We define

$$
f(z, \zeta, \theta, M)=|\ell(z, \zeta+i \theta M)|^{2}+|\theta M|^{2}\left|\left\langle\ell_{\zeta}^{\prime}(z, \zeta+i \theta M), M\right\rangle\right|^{2}
$$


As the simple-characteristic property holds in direction $M$ for all $M \in K$ and $z \in \bar{V}$, we have

$$
f(z, \zeta, \theta, M) \geq C>0, \quad(z, \zeta, \theta, M) \in \mathscr{C} .
$$

By homogeneity, we obtain

$$
f(z, \zeta, \theta, M) \geq C\left(\theta^{2}+|\zeta|^{2}\right)^{m}, \quad z \in \bar{V}, \zeta \in \mathbb{R}^{N}, \theta \in \mathbb{R}_{+}, M \in K .
$$

We compute the following Poisson bracket, with $\hat{\tau}\left(\varrho^{\prime}\right)=\tau d \varphi(z)$,

$$
\left\{\operatorname{Re} \ell\left(z, \zeta+i \hat{\tau}\left(\varrho^{\prime}\right)\right), \operatorname{Im} \ell\left(z, \zeta+i \hat{\tau}\left(\varrho^{\prime}\right)\right)\right\}=\frac{1}{2 i}\left\{\overline{\ell\left(z, \zeta+i \hat{\tau}\left(\varrho^{\prime}\right)\right)}, \ell\left(z, \zeta+i \hat{\tau}\left(\varrho^{\prime}\right)\right)\right\}=\Theta_{\ell, \varphi}(z, \zeta, \tau),
$$

with

$$
\begin{aligned}
\Theta_{\ell, \phi}(z, \zeta, t):= & t \sum_{j, k} \partial_{z_{j, k}}^{2} \phi(z) \partial_{\zeta_{j}} \ell(z, \zeta+i t d \phi(z)) \partial_{\zeta_{k}} \overline{\ell(z, \zeta+i t d \phi(z))} \\
& +\operatorname{Im} \sum_{j} \partial_{z_{j}} \ell(z, \zeta+i t d \phi(z)) \partial_{\zeta_{j}} \overline{\ell(z, \zeta+i t d \phi(z))} .
\end{aligned}
$$

Note that $\Theta_{\ell, \phi}(z, \zeta, t)$ is homogeneous of degree $2 m-1$ in $(\zeta, t)$. With $\varphi(z)=\exp \left(\gamma \psi_{\varepsilon}(z)\right)$ we obtain

$$
\Theta_{\ell, \varphi}(z, \zeta, \tau)=\Theta_{\ell, \psi_{\varepsilon}}\left(z, \zeta, \tilde{\tau}\left(\varrho^{\prime}\right)\right)+\gamma \tilde{\tau}\left(\varrho^{\prime}\right)\left|\left\langle\ell_{\zeta}^{\prime}\left(z, \zeta+i \hat{\tau}\left(\varrho^{\prime}\right)\right), \psi_{\varepsilon}^{\prime}(z)\right\rangle\right|^{2} .
$$

We thus find, with $f$ defined in (B.1),

(B.3)

$$
\begin{aligned}
\mid \ell(z, \zeta & \left.+i \hat{\tau}\left(\varrho^{\prime}\right)\right)\left.\right|^{2}+\tau \varphi(z)\left|\psi_{\varepsilon}^{\prime}(z)\right|^{2}\left\{\operatorname{Re} \ell\left(z, \zeta+i \hat{\tau}\left(\varrho^{\prime}\right)\right), \operatorname{Im} \ell\left(z, \zeta+i \hat{\tau}\left(\varrho^{\prime}\right)\right)\right\} \\
& =\left|\ell\left(z, \zeta+i \hat{\tau}\left(\varrho^{\prime}\right)\right)\right|^{2}+\tau \varphi(z)\left|\psi_{\varepsilon}^{\prime}(z)\right|^{2} \Theta_{\ell, \varphi}(z, \zeta, \tau) \\
& =\left|\ell\left(z, \zeta+i \hat{\tau}\left(\varrho^{\prime}\right)\right)\right|^{2}+\left|\tilde{\tau}\left(\varrho^{\prime}\right) \psi_{\varepsilon}^{\prime}(z)\right|^{2}\left|\left\langle\ell_{\zeta}^{\prime}\left(z, \zeta+i \hat{\tau}\left(\varrho^{\prime}\right)\right), \psi_{\varepsilon}^{\prime}(z)\right\rangle\right|^{2}+\tau \varphi(z)\left|\psi_{\varepsilon}^{\prime}(z)\right|^{2} \Theta_{\ell, \psi_{\varepsilon}}\left(z, \zeta, \tilde{\tau}\left(\varrho^{\prime}\right)\right) \\
& =f\left(z, \zeta, \tilde{\tau}\left(\varrho^{\prime}\right), \psi_{\varepsilon}^{\prime}(z)\right)+\tau \varphi(z)\left|\psi_{\varepsilon}^{\prime}(z)\right|^{2} \Theta_{\ell, \psi_{\varepsilon}}\left(z, \zeta, \tilde{\tau}\left(\varrho^{\prime}\right)\right) .
\end{aligned}
$$

Now, as $\psi_{\varepsilon}^{\prime}(z)$ remains in the compact set $K$, we find, by (B.2),

$$
f\left(z, \zeta, \tilde{\tau}\left(\varrho^{\prime}\right), \psi_{\varepsilon}^{\prime}(z)\right) \gtrsim\left(\hat{\tau}\left(\varrho^{\prime}\right)^{2}+|\zeta|^{2}\right)^{m} \gtrsim \lambda_{\tilde{\tau}}^{2 m},
$$

since $\left|\hat{\tau}\left(\varrho^{\prime}\right)\right|=\left|\psi_{\varepsilon}^{\prime}\right| \tilde{\tau}\left(\varrho^{\prime}\right) \geq C_{0} \tilde{\tau}\left(\varrho^{\prime}\right)$. The homogeneity of $\Theta_{\ell, \psi_{\varepsilon}}\left(z, \zeta, \tilde{\tau}\left(\varrho^{\prime}\right)\right)$ gives

$$
\left.|\tau \varphi(z)| \psi_{\varepsilon}^{\prime}(z)\right|^{2} \Theta_{\ell, \psi_{\varepsilon}}\left(z, \zeta, \tilde{\tau}\left(\varrho^{\prime}\right)\right) \mid \lesssim \gamma^{-1} \tilde{\tau}\left(\varrho^{\prime}\right) \lambda_{\tilde{\tau}}^{2 m-1} \lesssim \gamma^{-1} \lambda_{\tilde{\tau}}^{2 m}
$$

With (B.3) and (B.4), we obtain the result for $\gamma$ chosen sufficiently large.

We recall the definition of $q_{k}(\varrho)$ given in (4.24), we have $q_{k}(y)=p_{k}\left(z, \zeta+i \hat{\tau}\left(\varrho^{\prime}\right)\right)$ with $p_{k}(z, \zeta)=$ $(-1)^{k} i \sigma^{2}+\xi_{d}^{2}+r\left(x, \xi^{\prime}\right)$. From Proposition 2.3 and Proposition B.2, we have the following result, in any dimension $N \geq 2$, that is, $d \geq 1$.

Corollary B.3. Let $k=1$ or 2. There exist $C>0$ and $\gamma_{0} \geq 1$ such that

$$
\left|q_{k}(\varrho)\right|^{2}+\tau \varphi(z)\left|\psi_{\varepsilon}^{\prime}(z)\right|^{2}\left\{\operatorname{Re} q_{k}\left(\varrho^{\prime}\right), \operatorname{Im} q_{k}(\varrho)\right\} \geq C \lambda_{\tilde{\tau}}^{4}, \quad \varrho=(z, \zeta, \tau, \gamma, \varepsilon),
$$

for $z \in \bar{V}, \zeta \in \mathbb{R}^{N}, \tau \geq \tau_{*}, \gamma \geq \gamma_{0}$, and $\varepsilon \in[0,1]$, and where $\hat{\tau}\left(\varrho^{\prime}\right)=\tau \gamma \varphi(z) d \psi_{\varepsilon}(z)$.

B.3. Estimates for first-order factors. In this section, we shall assume that $\mathscr{U}_{0} \subset \mathcal{M}_{\mathrm{T}}$ is a conic open set where the symbol $q_{k}(\varrho)=p_{k}\left(z, \zeta+i \hat{\tau}\left(\varrho^{\prime}\right)\right)$ can be factorized into two smooth first-order terms,

$$
q_{k}(\varrho)=q_{k,-}(\varrho) q_{k,+}(\varrho), \quad q_{k, \pm}(\varrho)=\xi_{d}-\rho_{k, \pm}\left(\varrho^{\prime}\right) .
$$

By Lemma 4.18 we see that $q_{k,-}$ is elliptic, and $q_{k,+}$ may vanish. 
B.3.1. A root with a positive imaginary part: an elliptic estimate with a trace term. Here, we further assume that there exists a second conic open set $\mathscr{U}_{1} \subset \mathscr{U}_{0}$ such that $\operatorname{Im} \rho_{k,+}\left(\varrho^{\prime}\right) \gtrsim \lambda_{\mathrm{T}, \tilde{\tau}}$, for $\varrho^{\prime} \in \mathscr{U}_{1}$. We let $\chi, \underline{\chi} \in S\left(1, g_{\top}\right)$ be homogeneous of degree zero and such that

$$
\underline{\chi} \equiv 1 \text { on a conic neighborhood of } \operatorname{supp}(\chi), \quad \operatorname{supp}(\underline{\chi}) \subset \mathscr{U}_{1} \text {. }
$$

With $Q_{k,+}=D_{x_{d}}-\mathrm{Op}_{\mathrm{T}}{ }^{w}\left(\underline{x}^{2} \rho_{k,+}\right)$ we have the following estimation.

Lemma B.4. Let $\ell \in \mathbb{R}$ and $M \in \mathbb{N}$. There exist $\tau_{1} \geq \tau_{*}, \gamma_{1} \geq 1$, and $C>0$, such that

$$
\left\|\mathrm{Op}_{\mathrm{T}}(\chi) w\right\|_{1, \ell, \tilde{\tau}} \leq C\left(\left\|Q_{k,+} \mathrm{Op}_{\mathrm{T}}(\chi) w\right\|_{0, \ell, \tilde{\tau}}+\left|\operatorname{tr}\left(\mathrm{Op}_{\mathrm{T}}(\chi) w\right)\right|_{0, \ell+1 / 2, \tilde{\tau}}+\|w\|_{0,-M, \tilde{\tau}}\right),
$$

for $\tau \geq \tau_{1}, \gamma \geq \gamma_{1}, \varepsilon \in[0,1]$, and for $w \in \mathscr{S}\left(\overline{\mathbb{R}_{+}^{N}}\right)$.

Proof. We write $Q=A-i B$ with

$$
A=D_{x_{d}}-\mathrm{Op}_{\mathrm{T}}{ }^{w}\left(\underline{\chi}^{2} \operatorname{Re} \rho_{k,+}\right), \quad B=\mathrm{Op}_{\mathrm{T}}{ }^{w}\left(\underline{\chi}^{2} \operatorname{Im} \rho_{k,+}\right),
$$

both formally selfadjoint.

We use a pseudo-differential multiplier technique, following for instance [LL13] and compute, with $s=2 \ell+1$,

$$
\begin{aligned}
2 \operatorname{Re}( & \left.Q \mathrm{Op}_{\mathrm{T}}(\chi) w,-i \Lambda_{\mathrm{T}, \tilde{\tau}}^{s} \mathrm{Op}_{\mathrm{T}}(\chi) w\right)_{+} \\
= & -2 \operatorname{Re}\left(A \mathrm{Op}_{\mathrm{T}}(\chi) w, i \Lambda_{\mathrm{T}, \tilde{\tau}}^{s} \mathrm{Op}_{\mathrm{T}}(\chi) w\right)_{+}+2 \operatorname{Re}\left(B \mathrm{Op}_{\mathrm{T}}(\chi) w, \Lambda_{\mathrm{T}, \tilde{\tau}}^{s} \mathrm{Op}_{\mathrm{T}}(\chi) w\right)_{+} \\
= & -\left(i\left[A, \Lambda_{\mathrm{T}, \tilde{\tau}}^{s}\right] \mathrm{Op}_{\mathrm{T}}(\chi) w, \mathrm{Op}_{\mathrm{T}}(\chi) w\right)_{+}+2 \operatorname{Re}\left(B \mathrm{Op}_{\mathrm{T}}(\chi) w, \Lambda_{\mathrm{T}, \tilde{\tau}}^{s} \mathrm{Op}_{\mathrm{T}}(\chi) w\right)_{+} \\
& -\left(\Lambda_{\mathrm{T}, \tilde{\tau}}^{s} \mathrm{Op}_{\mathrm{T}}(\chi) w_{\mid x_{d}=0^{+}}, \mathrm{Op}_{\mathrm{T}}(\chi) w_{\mid x_{d}=0^{+}}\right)_{L^{2}\left(\mathbb{R}^{N-1}\right)} \\
\geq & 2 \operatorname{Re}\left(B \mathrm{Op}_{\mathrm{T}}(\chi) w, \Lambda_{\mathrm{T}, \tilde{\tau}}^{s} \mathrm{Op}_{\mathrm{T}}(\chi) w\right)_{+}-C \gamma^{2}\left\|\mathrm{Op}_{\mathrm{T}}(\chi) w\right\|_{0, \ell+1 / 2, \tilde{\tau}}^{2} \\
& -\left|\mathrm{Op}_{\mathrm{T}}(\chi) w_{\mid x_{d}=0^{+}}\right|_{0, \ell+1 / 2, \tilde{\tau}}^{2},
\end{aligned}
$$

which by the (microlocal) Gårding inequality of Proposition 4.16 yields, for any $M \in \mathbb{N}$, $\operatorname{Re}\left(\Lambda_{\mathrm{T}, \tilde{\tau}}^{\ell} Q \mathrm{Op}_{\mathrm{T}}(\chi) w,-i \Lambda_{\mathrm{T}, \tilde{\tau}}^{\ell+1} \mathrm{Op}_{\mathrm{T}}(\chi) w\right)_{+}+\left|\mathrm{Op}_{\mathrm{T}}(\chi) w_{\mid x_{d}=0^{+}}\right|_{0, \ell+1 / 2, \tilde{\tau}}^{2}+\|w\|_{0,-M, \tilde{\tau}}^{2} \gtrsim\left\|\mathrm{Op}_{\mathrm{T}}(\chi) w\right\|_{0, \ell+1, \tilde{\tau}}^{2}$, for $\tau$ and $\gamma$ chosen sufficiently large. Then, with the Young inequality, we obtain

$$
\left\|Q \mathrm{Op}_{\mathrm{T}}(\chi) w\right\|_{0, \ell, \tilde{\tau}}+\left|\mathrm{Op}_{\mathrm{T}}(\chi) w_{\mid x_{d}=0^{+}}\right|_{0, \ell+1 / 2, \tilde{\tau}}+\|w\|_{0,-M, \tilde{\tau}} \gtrsim\left\|\mathrm{Op}_{\mathrm{T}}(\chi) w\right\|_{0, \ell+1, \tilde{\tau}} .
$$

Finally, observing that we have

$$
\left\|D_{x_{d}} \mathrm{Op}_{\mathrm{T}}(\chi) w\right\|_{0, \ell, \tilde{\tau}} \lesssim\left\|Q \mathrm{Op}_{\mathrm{T}}(\chi) w\right\|_{0, \ell, \tilde{\tau}}+\left\|\mathrm{Op}_{\mathrm{T}}(\chi) w\right\|_{0, \ell+1, \tilde{\tau}}
$$

allows one to conclude the proof.

B.3.2. Transmitted sub-ellipticity. In $\mathscr{U}_{0}$ where $q_{k}(\varrho)$ is smoothly factorized, $q_{k}(\varrho)=q_{k,-}(\varrho) q_{k,+}(\varrho)$, we now describe how the sub-ellipticity property of Corollary B.3 is "transmitted" to the nonelliptic factor $q_{k,+}$.

Proposition B.5. Let $k=1$ or 2. There exist $\gamma_{0} \geq 1, \alpha_{0}>0$, and $C>0$ such that

$$
\alpha \gamma \tilde{\tau}^{-1}\left|\operatorname{Im} \rho_{k,+}\right|^{2}+\left\{\xi_{d}-\operatorname{Re} \rho_{k,+},-\operatorname{Im} \rho_{k,+}\right\} \geq C \gamma \tilde{\tau}^{-1} \lambda_{\mathbf{T}, \tilde{\tau}}^{2}, \quad \varrho^{\prime} \in \mathscr{U}_{0},
$$

for $\gamma \geq \gamma_{0}$ and $\alpha \geq \alpha_{0}$.

Proof. We write

$$
2 i\left\{\operatorname{Re} q_{k}, \operatorname{Im} q_{k}\right\}=\left\{\overline{q_{k}}, q_{k}\right\}=\left|q_{k,-}\right|^{2}\left\{\overline{q_{k,+}}, q_{k,+}\right\}+\left|q_{k,+}\right|^{2}\left\{\overline{q_{k,-}}, q_{k,-}\right\}+2 i \operatorname{Im}\left(\left\{\overline{q_{k,-}}, q_{k,+}\right\} \overline{q_{k,+}} q_{k,-}\right),
$$
yielding

$\left\{\operatorname{Re} q_{k}, \operatorname{Im} q_{k}\right\}=\left|q_{k,-}\right|^{2}\left\{\operatorname{Re} q_{k,+}, \operatorname{Im} q_{k,+}\right\}+\left|q_{k,+}\right|^{2}\left\{\operatorname{Re} q_{k,-}, \operatorname{Im} q_{k,-}\right\}+\operatorname{Im}\left(\left\{\overline{q_{k,-}}, q_{k,+}\right\} \overline{q_{k,+}} q_{k,-}\right)$. 
We write, for $M>0$,

$$
\begin{aligned}
\left|q_{k,+}\right|^{2}\left|\left\{\operatorname{Re} q_{k,-}, \operatorname{Im} q_{k,-}\right\}\right|+\left|\operatorname{Im}\left(\left\{\overline{q_{k,-}}, q_{k,+}\right\} \overline{q_{k,+}} q_{k,-}\right)\right| & \leq C\left(\gamma \lambda_{\tilde{\tau}}\left|q_{k,+}\right|^{2}+\gamma \lambda_{\tilde{\tau}}^{2}\left|q_{k,+}\right|\right) \\
& \leq C^{\prime}(1+M) \gamma \lambda_{\tilde{\tau}}\left|q_{k,+}\right|^{2}+C^{\prime} M^{-1} \gamma \lambda_{\tilde{\tau}}^{3} .
\end{aligned}
$$

For $M>0$ and $\gamma_{0} \geq 1$ chosen sufficiently large we obtain, with Corollary B.3,

$$
\left|q_{k,-}(\varrho)\right|^{2}\left(\left|q_{k,+}(\varrho)\right|^{2}+\tau \varphi(z)\left|\psi_{\varepsilon}^{\prime}(z)\right|^{2}\left\{\operatorname{Re} q_{k,+}, \operatorname{Im} q_{k,+}\right\}(\varrho)\right) \geq C \lambda_{\tilde{\tau}}^{4}-C^{\prime}(1+M) \tilde{\tau} \lambda_{\tilde{\tau}}\left|q_{k,+}\right|^{2},
$$

In $\mathscr{U}_{0}$ we have $\left|q_{k,-}(\varrho)\right| \asymp \lambda_{\tilde{\tau}}$, as $q_{k,-}$ is elliptic which gives

$$
\alpha\left|q_{k,+}(\varrho)\right|^{2}+\tau \varphi(z)\left|\psi_{\varepsilon}^{\prime}(z)\right|^{2}\left\{\operatorname{Re} q_{k,+}, \operatorname{Im} q_{k,+}\right\}(\varrho) \geq C \lambda_{\tilde{\tau}}^{2}, \quad \varrho^{\prime} \in \mathscr{U}_{0}, \xi_{d} \in \mathbb{R},
$$

for $\alpha>0$ chosen sufficiently large. If we now choose $\xi_{d}=\operatorname{Re} \rho_{k,+}\left(\varrho^{\prime}\right)$ we then obtain the result.

B.3.3. A root with a vanishing imaginary part: a sub-elliptic estimate with a trace term. Here, we consider as above a conic open set $\mathscr{U}_{0} \subset \mathcal{M}_{\mathrm{T}}$, such that the symbol $q_{k}(\varrho)=p_{k}\left(z, \zeta+i \hat{\tau}\left(\varrho^{\prime}\right)\right)$ can be factorized into two smooth first-order terms, $q_{k}(\varrho)=q_{k,-}(\varrho) q_{k,+}(\varrho)$. We let $\chi, \underline{\chi} \in S\left(1, g_{\mathrm{T}}\right)$ be as above and we recall that $Q_{k,+}:=D_{x_{d}}-\mathrm{Op}_{\mathrm{T}}{ }^{w}\left(\chi^{2} \rho_{k,+}\right)$. We have the following lemma.

Lemma B.6. Let $\ell, m \in \mathbb{R}$ and $M \in \mathbb{N}$. There exist $\tau_{1} \geq \tau_{*}, \gamma_{1} \geq 1$, and $C>0$, such that

$$
\gamma^{1 / 2}\left\|\tilde{\tau}^{m-1 / 2} \mathrm{Op}_{\mathrm{T}}(\chi) w\right\|_{1, \ell, \tilde{\tau}} \leq C\left(\left\|\tilde{\tau}^{m} Q_{k,+} \mathrm{Op}_{\mathrm{T}}(\chi) w\right\|_{0, \ell, \tilde{\tau}}+\left|\operatorname{tr}\left(\tilde{\tau}^{m} \mathrm{Op}_{\mathrm{T}}(\chi) w\right)\right|_{0, \ell+1 / 2, \tilde{\tau}}+\|w\|_{0,-M, \tilde{\tau}}\right),
$$

for $\tau \geq \tau_{1}, \gamma \geq \gamma_{1}, \varepsilon \in[0,1]$, and for $w \in \mathscr{S}\left(\overline{\mathbb{R}_{+}^{N}}\right)$.

Proof. For concision, we write $Q$ in place of $Q_{k,+}$. We decompose $Q$ according to $Q=A+i B$ with

(B.8) $\quad A=D_{x_{d}}-\mathrm{Op}_{\mathrm{T}}{ }^{w}\left(\underline{\chi}^{2} \operatorname{Re} \rho_{k,+}\right) \in \Psi_{\tilde{\tau}}^{1,0}, \quad B=-\mathrm{Op}_{\mathrm{T}}{ }^{w}\left(\underline{\chi}^{2} \operatorname{Im} \rho_{k,+}\right) \in \Psi_{\tilde{\tau}}^{0,1}=\Psi\left(\lambda_{\mathrm{T}, \tilde{\tau}}, g_{\mathrm{T}}\right)$.

Observe that both $A$ and $B$ are formally selfadjoint.

We set $w_{\ell, m}=\tilde{\tau}^{m} \Lambda_{\mathrm{T}, \tilde{\tau}}^{\ell} \mathrm{Op}_{\mathrm{T}}(\chi) w$ and compute

$$
\left\|Q w_{\ell, m}\right\|_{+}^{2}=\left\|(A+i B) w_{\ell, m}\right\|_{+}^{2}=\left\|A w_{\ell, m}\right\|_{+}^{2}+\left\|B w_{\ell, m}\right\|_{+}^{2}+2 \operatorname{Re}\left(A w_{\ell, m}, i B w_{\ell, m}\right)_{+}
$$

From the form of $A$ and $B$ given in (B.8) we find

$$
2 \operatorname{Re}\left(A w_{\ell, m}, i B w_{\ell, m}\right)_{+}=i\left([A, B] w_{\ell, m}, w_{\ell, m}\right)_{+}-\left(\mathrm{Op}^{w}{ }^{w}\left(\underline{\chi}^{2} \operatorname{Im} \rho_{k,+}\right) w_{\ell, m \mid x_{d}=0^{+}}, w_{\ell, m \mid x_{d}=0^{+}}\right)_{L^{2}\left(\mathbb{R}^{N-1}\right)} .
$$

yielding, with (B.9),

$$
\begin{aligned}
& \left\|Q w_{\ell, m}\right\|_{+}^{2}+\left|\operatorname{tr}\left(\tilde{\tau}^{m} \operatorname{Op}_{\mathrm{T}}(\chi) w\right)\right|_{0, \ell+1 / 2, \tilde{\tau}}^{2} \\
& \quad \gtrsim\left\|A w_{\ell, m}\right\|_{+}^{2}+\left\|B w_{\ell, m}\right\|_{+}^{2}+i\left([A, B] w_{\ell, m}, w_{\ell, m}\right)_{+} \\
& \quad \gtrsim\left\|A w_{\ell, m}\right\|_{+}^{2}+\left(\left(\alpha \gamma \tilde{\tau}^{-1} B^{2}+i[A, B]\right) w_{\ell, m}, w_{\ell, m}\right)_{+} \\
& \quad \gtrsim\left\|A w_{\ell, m}\right\|_{+}^{2}+\left(\Lambda_{\mathrm{T}, \tilde{\tau}^{\ell}}^{\ell}\left(\alpha \gamma \tilde{\tau}^{-1} B^{2}+i[A, B]\right) \tilde{\tau}^{m} \Lambda_{\mathrm{T}, \tilde{\tau}}^{\ell} \mathrm{Op}_{\mathrm{T}}(\chi) w, \mathrm{Op}_{\mathrm{T}}(\chi) w\right)_{+},
\end{aligned}
$$

for $\alpha=\alpha_{0}$ with $\alpha_{0}$ given by Proposition B.5, and for $\tau$ such that $\alpha \gamma \tilde{\tau}^{-1} \leq 1$. As the principal symbol of $\Lambda_{\mathrm{T}, \tilde{\tau}}^{\ell} \tilde{\tau}^{m}\left(\alpha \gamma \tilde{\tau}^{-1} B^{2}+i[A, B]\right) \tilde{\tau}^{m} \Lambda_{\mathrm{T}, \tilde{\tau}}^{\ell}$ is given, in a conic neighborhood of $\operatorname{supp}(\chi)$, where $\underline{\chi} \equiv 1$, by

$$
\tilde{\tau}^{2 m} \lambda_{\mathrm{T}, \tilde{\tau}}^{2 \ell}\left(\alpha \gamma \tilde{\tau}^{-1}\left(\operatorname{Im} \rho_{k,+}\right)^{2}+\left\{\xi_{d}-\operatorname{Re} \rho_{k,+},-\operatorname{Im} \rho_{k,+}\right\}\right) \in S\left(\gamma \tilde{\tau}^{2 m-1} \lambda_{\mathrm{T}, \tilde{\tau}}^{2+2 \ell}, g_{\mathrm{T}}\right),
$$


then Proposition B.5 and the (microlocal) Gårding inequality of Proposition 4.16 yield, for any $M \in N$, by choosing $\tau$ and $\gamma$ sufficiently large,

$$
\left\|Q w_{\ell, m}\right\|_{+}+\left|\operatorname{tr}\left(\tilde{\tau}^{m} \operatorname{Op}_{\mathrm{T}}(\chi) w\right)\right|_{0, \ell+1 / 2, \tilde{\tau}}+\|w\|_{0,-M} \gtrsim\left\|A w_{\ell, m}\right\|_{+}+\gamma^{1 / 2}\left\|\tilde{\tau}^{m-1 / 2} \operatorname{Op}_{\mathrm{T}}(\chi) w\right\|_{0,1+\ell, \tilde{\tau}} .
$$

From the form of $A$ in (B.8) we have

$$
\begin{aligned}
\gamma^{1 / 2}\left\|\tilde{\tau}^{-1 / 2} D_{x_{d}} w_{\ell, m}\right\|_{+} & \lesssim\left\|A w_{\ell, m}\right\|_{+}+\gamma^{1 / 2}\left\|\tilde{\tau}^{-1 / 2} w_{\ell, m}\right\|_{0,1, \tilde{\tau}} \\
& \lesssim\left\|A w_{\ell, m}\right\|_{+}+\gamma^{1 / 2}\left\|\tilde{\tau}^{m-1 / 2} \mathrm{Op}_{\mathrm{T}}(\chi) w\right\|_{0,1+\ell, \tilde{\tau}}
\end{aligned}
$$

We thus obtain

$$
\begin{aligned}
& \|Q w \ell, m\|_{+}+\left|\operatorname{tr}\left(\tilde{\tau}^{m} \mathrm{Op}_{\mathrm{T}}(\chi) w\right)\right|_{0, \ell+1 / 2, \tilde{\tau}}+\|w\|_{0,-M} \\
& \quad \gtrsim \gamma^{1 / 2}\left(\left\|\tilde{\tau}^{m-1 / 2} \mathrm{Op}_{\mathrm{T}}(\chi) w\right\|_{0,1+\ell, \tilde{\tau}}+\left\|\tilde{\tau}^{-1 / 2} D_{x_{d}} w_{\ell, m}\right\|_{+}\right) \\
& \quad \gtrsim \gamma^{1 / 2}\left\|\tilde{\tau}^{m-1 / 2} \mathrm{Op}_{\mathrm{T}}(\chi) w\right\|_{1, \ell, \tilde{\tau}},
\end{aligned}
$$

by choosing $\tau$ sufficiently large and using Lemma 4.14. This concludes the proof.

B.4. Estimate concatenations. Let $\mathscr{U}_{0}$ be on conic open set of $\mathcal{M}_{\mathrm{T}}$. Let $\underline{\chi}\left(\varrho^{\prime}\right) \in S\left(1, g_{\mathrm{T}}\right)$ be homogeneous of degree zero such that $\operatorname{supp}(\chi) \subset \mathscr{U}_{0}$. Let $\rho^{(k)}\left(\varrho^{\prime}\right) \in S\left(\lambda_{\mathrm{T}, \tilde{\tau}}, g_{\mathrm{T}}\right), k=1,2$, be homogeneous of degree one in $\mathscr{U}_{0}$ and define $Q^{(k)}=D_{x_{d}}-\mathrm{Op}^{w}{ }^{w}\left(\chi^{2} \rho^{(k)}\right)$. The operators $Q_{k, \pm}$, $k=1,2$, defined in what precedes and in Section 4 are of this form. Above, for such operators, we proved some microlocal estimates of the form

$$
\begin{aligned}
& \gamma^{\alpha_{k} / 2}\left\|\tilde{\tau}^{\alpha_{k}(m-1 / 2)} \mathrm{Op}_{\mathrm{T}}(\chi) w\right\|_{1, \ell, \tilde{\tau}}+\delta_{k}\left|\operatorname{tr}\left(\tilde{\tau}^{m \alpha_{k}} \mathrm{Op}_{\mathrm{T}}(\chi) w\right)\right|_{0, \ell+1 / 2, \tilde{\tau}} \\
& \quad \leq C\left(\left\|\tilde{\tau}^{m \alpha_{k}} Q^{(k)} \mathrm{Op}_{\mathrm{T}}(\chi) w\right\|_{0, \ell, \tilde{\tau}}+\left(1-\delta_{k}\right)\left|\operatorname{tr}\left(\tilde{\tau}^{m \alpha_{k}} \mathrm{Op}_{\mathrm{T}}(\chi) w\right)\right|_{0, \ell+1 / 2, \tilde{\tau}}+\|w\|_{0,-M, \tilde{\tau}}\right),
\end{aligned}
$$

with $\delta_{k}=\left(1-\alpha_{k}\right)\left(1-\beta_{k}\right)$ and $\alpha_{k}, \beta_{k} \in\{0,1\}, \ell, m \in \mathbb{R}$, and where $\chi \in S\left(1, g_{\top}\right)$, homogeneous of degree zero and such that $\underline{\chi} \equiv 1$ on a conic neighborhood of $\operatorname{supp}(\chi)$.

If $\alpha_{k}=0$ and $\beta_{k}=0$ the estimate reads

$$
\left\|\mathrm{Op}_{\mathrm{T}}(\chi) w\right\|_{1, \ell, \tilde{\tau}}+\left|\operatorname{tr}\left(\mathrm{Op}_{\mathrm{T}}(\chi) w\right)\right|_{0, \ell+1 / 2, \tilde{\tau}} \leq C\left(\left\|Q^{(k)} \mathrm{Op}_{\mathrm{T}}(\chi) w\right\|_{0, \ell, \tilde{\tau}}+\|w\|_{0,-M, \tilde{\tau}}\right) .
$$

This is a perfect elliptic estimate that holds if $\rho^{(k)}$ is in the lower half complex plane-see Lemma B.1.

If $\alpha_{k}=0$ and $\beta_{k}=1$ the estimate reads

$$
\left\|\mathrm{Op}_{\mathrm{T}}(\chi) w\right\|_{1, \ell, \tilde{\tau}} \leq C\left(\left\|Q^{(k)} \mathrm{Op}_{\mathrm{T}}(\chi) w\right\|_{0, \ell, \tilde{\tau}}+\left|\operatorname{tr}\left(\mathrm{Op}_{\mathrm{T}}(\chi) w\right)\right|_{0, \ell+1 / 2, \tilde{\tau}}+\|w\|_{0,-M, \tilde{\tau}}\right) .
$$

This is an elliptic estimate, yet with a trace observation term in the r.h.s., that holds if $\rho^{(k)}$ is in the upper half complex plane-see Lemma B.4.

Finally, if $\alpha_{k}=1$, independently of the value of $\beta_{k}$ we have

$$
\gamma^{1 / 2}\left\|\tilde{\tau}^{m-1 / 2} \mathrm{Op}_{\mathrm{T}}(\chi) w\right\|_{1, \ell, \tilde{\tau}} \leq C\left(\left\|\tilde{\tau}^{m} Q^{(k)} \mathrm{Op}_{\mathrm{T}}(\chi) w\right\|_{0, \ell, \tilde{\tau}}+\left|\operatorname{tr}\left(\tilde{\tau}^{m} \mathrm{Op}_{\mathrm{T}}(\chi) w\right)\right|_{0, \ell+1 / 2, \tilde{\tau}}+\|w\|_{0,-M, \tilde{\tau}}\right) .
$$

This estimate is characterized by the loss of a half derivative and a boundary observation term in the r.h.s.; such an estimate is proven in Lemma B.6 when the root $\rho^{(k)}$ may cross the real axis.

We shall now describe how such estimates can be concatenated, as this is often done in the course of the proof of Theorem 4.17.

Proposition B.7. Let $\ell \in \mathbb{R}$ and $M \in \mathbb{N}$. Let $Q^{(k)}$ be defined as above, for $k=1,2$. Let $\tau_{0} \geq \tau_{*}$, $\gamma_{0} \geq 1$ and $C>0$ such that estimate (B.10) holds, with $\ell, m \in \mathbb{R}$, with $\alpha_{k}, \beta_{k} \in\{0,1\}$, for both $k=1$ and 2 , for $\tau \geq \tau_{0}, \gamma \geq \gamma_{0}, \varepsilon \in[0,1]$, and for $w \in \mathscr{S}\left(\overline{\mathbb{R}_{+}^{N}}\right)$. We assume that $\alpha_{1} \leq \alpha_{2}$ and $1-\delta_{1} \leq 1-\delta_{2}$. 
Let $\chi \in S\left(1, g_{\top}\right)$, be homogeneous of degree zero and such that $\chi \equiv 1$ on $\operatorname{supp}(\chi)$. There exist $\tau_{1} \geq \tau_{*}, \gamma_{1} \geq 1$ and $C>0$ such that the following estimate for the second-order operator $Q^{(1)} Q^{(2)}$ holds,

$$
\begin{aligned}
\gamma^{\left(\alpha_{1}+\alpha_{2}\right) / 2}\left\|\tilde{\tau}^{-\left(\alpha_{1}+\alpha_{2}\right) / 2} \mathrm{Op}_{\mathrm{T}}(\chi) w\right\|_{2, \ell, \tilde{\tau}}+\left|\operatorname{tr}\left(\mathrm{Op}_{\mathrm{T}}(\chi) w\right)\right|_{1, \ell+1 / 2, \tilde{\tau}} \\
\leq C\left(\left\|Q^{(1)} Q^{(2)} \mathrm{Op}_{\mathrm{T}}(\chi) w\right\|_{0, \ell, \tilde{\tau}}+\left(1-\delta_{1}\right)\left|\operatorname{tr}\left(\mathrm{Op}_{\mathrm{T}}(\chi) w\right)\right|_{1, \ell+1 / 2, \tilde{\tau}}\right. \\
\left.\quad+\left(1-\delta_{2}\right) \gamma^{\alpha_{1} / 2}\left|\operatorname{tr}\left(\tilde{\tau}^{-\alpha_{1} / 2} \mathrm{Op}_{\mathrm{T}}(\chi) w\right)\right|_{0, \ell+3 / 2, \tilde{\tau}}+\|w\|_{2,-M, \tilde{\tau}}\right),
\end{aligned}
$$

for $\tau \geq \tau_{1}, \gamma \geq \gamma_{1}, \varepsilon \in[0,1]$, and for $w \in \mathscr{S}\left(\overline{\mathbb{R}_{+}^{N}}\right)$.

Note that the assumptions made on $\alpha_{k}$ and $1-\delta_{k}, k=1,2$, imply that $Q^{(1)}$ yields an estimate of better quality than that associated with $Q^{(2)}$.

Proof. We introduce $\chi_{1} \in S\left(1, g_{\mathrm{T}}\right)$ that is such that $\chi_{1} \equiv 1$ on $\operatorname{supp}(\chi)$ and $\underline{\chi} \equiv 1$ on $\operatorname{supp}\left(\chi_{1}\right)$. For concision, we write $\Xi=\mathrm{Op}_{\mathrm{T}}(\chi)$ and $\Xi_{1}=\mathrm{Op}_{\mathrm{T}}\left(\chi_{1}\right)$. Here, $M$ will denote an arbitrary large integer whose value may change from one line to the other.

Using $Q^{(2)} \Xi w$ as the unknown function in the estimate (B.10) for $Q^{(1)}$, with $m=0$ gives,

$$
\begin{aligned}
& \gamma^{\alpha_{1} / 2}\left\|\tilde{\tau}^{-\alpha_{1} / 2} Q^{(2)} \Xi w\right\|_{1, \ell, \tilde{\tau}}+\delta_{1}\left|\operatorname{tr}\left(Q^{(2)} \Xi w\right)\right|_{0, \ell+1 / 2, \tilde{\tau}} \\
& \quad \lesssim \gamma^{\alpha_{1} / 2}\left\|\tilde{\tau}^{-\alpha_{1} / 2} \Xi_{1} Q^{(2)} \Xi w\right\|_{1, \ell, \tilde{\tau}}+\delta_{1}\left|\operatorname{tr}\left(\Xi_{1} Q^{(2)} \Xi w\right)\right|_{0, \ell+1 / 2, \tilde{\tau}} \\
& \quad+\|w\|_{1,-M, \tilde{\tau}}+|\operatorname{tr}(w)|_{1,-M, \tilde{\tau}} \\
& \lesssim\left\|Q^{(1)} \Xi_{1} Q^{(2)} \Xi w\right\|_{0, \ell, \tilde{\tau}}+\left(1-\delta_{1}\right)\left|\operatorname{tr}\left(\Xi_{1} Q^{(2)} \Xi w\right)\right|_{0, \ell+1 / 2, \tilde{\tau}}+\|w\|_{1,-M, \tilde{\tau}}+|\operatorname{tr}(w)|_{1,-M, \tilde{\tau}} \\
& \lesssim\left\|Q^{(1)} Q^{(2)} \Xi w\right\|_{0, \ell, \tilde{\tau}}+\left(1-\delta_{1}\right)|\operatorname{tr}(\Xi w)|_{1, \ell+1 / 2, \tilde{\tau}}+\|w\|_{2,-M, \tilde{\tau}}
\end{aligned}
$$

Observe now that we can write, using that $D_{x_{d}}-Q^{(2)} \in \Psi\left(\lambda_{\mathrm{T}, \tilde{\tau}}, g_{\mathrm{T}}\right)$,

$$
\begin{aligned}
\left(1-\delta_{2}\right) & \gamma^{\alpha_{1} / 2}\left|\operatorname{tr}\left(\tilde{\tau}^{-\alpha_{1} / 2} \Xi w\right)\right|_{1, \ell+1 / 2, \tilde{\tau}} \\
& \lesssim \delta_{1} \gamma^{\alpha_{1} / 2}\left|\operatorname{tr}\left(\tilde{\tau}^{-\alpha_{1} / 2} Q^{(2)} \Xi w\right)\right|_{0, \ell+1 / 2, \tilde{\tau}}+\left(1-\delta_{1}\right) \gamma^{\alpha_{1} / 2}\left|\operatorname{tr}\left(\tilde{\tau}^{-\alpha_{1} / 2} \Xi w\right)\right|_{1, \ell+1 / 2, \tilde{\tau}} \\
& +\left(1-\delta_{2}\right) \gamma^{\alpha_{1} / 2}\left|\operatorname{tr}\left(\tilde{\tau}^{-\alpha_{1} / 2} \Xi w\right)\right|_{0, \ell+3 / 2, \tilde{\tau}} \\
& \lesssim \delta_{1}\left|\operatorname{tr}\left(Q^{(2)} \Xi w\right)\right|_{0, \ell+1 / 2, \tilde{\tau}}+\left(1-\delta_{1}\right)|\operatorname{tr}(\Xi w)|_{1, \ell+1 / 2, \tilde{\tau}}+\left(1-\delta_{2}\right) \gamma^{\alpha_{1} / 2}\left|\operatorname{tr}\left(\tilde{\tau}^{-\alpha_{1} / 2} \Xi w\right)\right|_{0, \ell+3 / 2, \tilde{\tau}} .
\end{aligned}
$$

With this estimate and (B.11), we thus obtain

$$
\begin{aligned}
& \gamma^{\alpha_{1} / 2}\left(\left\|\tilde{\tau}^{-\alpha_{1} / 2} Q^{(2)} \Xi w\right\|_{1, \ell, \tilde{\tau}}+\left(1-\delta_{2}\right)\left|\operatorname{tr}\left(\tilde{\tau}^{-\alpha_{1} / 2} \Xi w\right)\right|_{1, \ell+1 / 2, \tilde{\tau}}\right) \\
& \quad \lesssim\left\|Q^{(1)} Q^{(2)} \Xi w\right\|_{0, \ell, \tilde{\tau}}+\left(1-\delta_{1}\right)|\operatorname{tr}(\Xi w)|_{1, \ell+1 / 2, \tilde{\tau}}+\left(1-\delta_{2}\right) \gamma^{\alpha_{1} / 2}\left|\operatorname{tr}\left(\tilde{\tau}^{-\alpha_{1} / 2} \Xi w\right)\right|_{0, \ell+3 / 2, \tilde{\tau}} \\
& \quad+\|w\|_{2,-M, \tilde{\tau}}
\end{aligned}
$$

Up to creating error terms, we shall now modify this inequality to be able to apply the estimate (B.10) associated with $Q^{(2)}$. We write

$$
\begin{aligned}
& \left\|\tilde{\tau}^{-\alpha_{1} / 2} Q^{(2)} \Xi_{1} D_{x_{d}} \Xi w\right\|_{0, \ell, \tilde{\tau}}+\left(1-\delta_{2}\right)\left|\operatorname{tr}\left(\tilde{\tau}^{-\alpha_{1} / 2} \Xi_{1} D_{x_{d}} \Xi w\right)\right|_{0, \ell+1 / 2, \tilde{\tau}} \\
& \quad \lesssim\left\|\tilde{\tau}^{-\alpha_{1} / 2} Q^{(2)} D_{x_{d}} \Xi w\right\|_{0, \ell, \tilde{\tau}}+\left(1-\delta_{2}\right)\left|\operatorname{tr}\left(\tilde{\tau}^{-\alpha_{1} / 2} D_{x_{d}} \Xi w\right)\right|_{0, \ell+1 / 2, \tilde{\tau}}+\|w\|_{1,-M, \tilde{\tau}}+|\operatorname{tr}(w)|_{1,-M, \tilde{\tau}} \\
& \quad \lesssim\left\|\tilde{\tau}^{-\alpha_{1} / 2} Q^{(2)} \Xi w\right\|_{1, \ell, \tilde{\tau}}+\left(1-\delta_{2}\right)\left|\operatorname{tr}\left(\tilde{\tau}^{-\alpha_{1} / 2} \Xi w\right)\right|_{1, \ell+1 / 2, \tilde{\tau}}+\|w\|_{1,-M, \tilde{\tau}}+|\operatorname{tr}(w)|_{1,-M, \tilde{\tau}} \\
& \quad+\gamma\left\|\tilde{\tau}^{-\alpha_{1} / 2} \Xi w\right\|_{1, \ell, \tilde{\tau}},
\end{aligned}
$$


using that $\left[D_{x_{d}}, Q^{(2)}\right] \in \gamma \Psi_{\tilde{\tau}}^{1,0}$ and using Lemma 4.14. Hence with (B.12) we have

$$
\begin{aligned}
& \gamma^{\alpha_{1} / 2}\left(\left\|\tilde{\tau}^{-\alpha_{1} / 2} Q^{(2)} \Xi_{1} D_{x_{d}} \Xi w\right\|_{0, \ell, \tilde{\tau}}+\left(1-\delta_{2}\right)\left|\operatorname{tr}\left(\tilde{\tau}^{-\alpha_{1} / 2} \Xi_{1} D_{x_{d}} \Xi w\right)\right|_{0, \ell+1 / 2, \tilde{\tau}}\right. \\
& \left.\quad+\left\|\tilde{\tau}^{-\alpha_{1} / 2} Q^{(2)} \Xi w\right\|_{0, \ell+1, \tilde{\tau}}+\left(1-\delta_{2}\right)\left|\operatorname{tr}\left(\tilde{\tau}^{-\alpha_{1} / 2} \Xi w\right)\right|_{0, \ell+3 / 2, \tilde{\tau}}\right) \\
& \lesssim \gamma^{\alpha_{1} / 2}\left(\left\|\tilde{\tau}^{-\alpha_{1} / 2} Q^{(2)} \Xi w\right\|_{1, \ell, \tilde{\tau}}+\left(1-\delta_{2}\right)\left|\operatorname{tr}\left(\tilde{\tau}^{-\alpha_{1} / 2} \Xi w\right)\right|_{1, \ell+1 / 2, \tilde{\tau}}\right. \\
& \left.\quad+\gamma\left\|\tilde{\tau}^{-\alpha_{1} / 2} \Xi w\right\|_{1, \ell, \tilde{\tau}}\right)+\|w\|_{1,-M, \tilde{\tau}}+|\operatorname{tr}(w)|_{1,-M, \tilde{\tau}} \\
& \lesssim\left\|Q^{(1)} Q^{(2)} \Xi w\right\|_{0, \ell, \tilde{\tau}}+\left(1-\delta_{1}\right)|\operatorname{tr}(\Xi w)|_{1, \ell+1 / 2, \tilde{\tau}}+\left(1-\delta_{2}\right) \gamma^{\alpha_{1} / 2}\left|\operatorname{tr}\left(\tilde{\tau}^{-\alpha_{1} / 2} \Xi w\right)\right|_{0, \ell+3 / 2, \tilde{\tau}} \\
& \quad+\gamma^{1+\alpha_{1} / 2}\left\|\tilde{\tau}^{-\alpha_{1} / 2} \Xi w\right\|_{1, \ell, \tilde{\tau}}+\|w\|_{2,-M, \tilde{\tau}}
\end{aligned}
$$

We write, with Lemma 4.14, for $\tau$ chosen sufficiently large,

$$
\begin{aligned}
\left\|\tilde{\tau}^{-\left(\alpha_{1}+\alpha_{2}\right) / 2} \Xi w\right\|_{2, \ell, \tilde{\tau}} & \asymp\left\|\tilde{\tau}^{-\left(\alpha_{1}+\alpha_{2}\right) / 2} D_{x_{d}} \Xi w\right\|_{1, \ell, \tilde{\tau}}+\left\|\tilde{\tau}^{-\left(\alpha_{1}+\alpha_{2}\right) / 2} \Xi w\right\|_{1, \ell+1, \tilde{\tau}} \\
& \lesssim\left\|\tilde{\tau}^{-\left(\alpha_{1}+\alpha_{2}\right) / 2} \Xi_{1} D_{x_{d}} \Xi w\right\|_{1, \ell, \tilde{\tau}}+\left\|\tilde{\tau}^{-\left(\alpha_{1}+\alpha_{2}\right) / 2} \Xi w\right\|_{1, \ell+1, \tilde{\tau}}+\|w\|_{2,-M, \tilde{\tau}},
\end{aligned}
$$

and

(B.15)

$$
\begin{aligned}
\left|\operatorname{tr}\left(\tilde{\tau}^{-\alpha_{1} / 2} \Xi w\right)\right|_{1, \ell+1 / 2, \tilde{\tau}} & \asymp\left|\operatorname{tr}\left(\tilde{\tau}^{-\alpha_{1} / 2} D_{x_{d}} \Xi w\right)\right|_{0, \ell+1 / 2, \tilde{\tau}}+\left|\operatorname{tr}\left(\tilde{\tau}^{-\alpha_{1} / 2} \Xi w\right)\right|_{0, \ell+3 / 2, \tilde{\tau}} \\
& \lesssim\left|\operatorname{tr}\left(\tilde{\tau}^{-\alpha_{1} / 2} \Xi_{1} D_{x_{d}} \Xi w\right)\right|_{0, \ell+1 / 2, \tilde{\tau}}+\left|\operatorname{tr}\left(\tilde{\tau}^{-\alpha_{1} / 2} \Xi w\right)\right|_{0, \ell+3 / 2, \tilde{\tau}}+|\operatorname{tr}(w)|_{1,-M, \tilde{\tau}} .
\end{aligned}
$$

Applying now estimate (B.10) associated with $Q^{(2)}$ to $D_{x_{d}} \Xi w$ and $w$, with $m=-\alpha_{1} / 2$, using that $\alpha_{1}=\alpha_{1} \alpha_{2}$, we obtain

(B.16)

$$
\begin{aligned}
& \gamma^{\left(\alpha_{1}+\alpha_{2}\right) / 2}\left\|\tilde{\tau}^{-\left(\alpha_{1}+\alpha_{2}\right) / 2} \Xi_{1} D_{x_{d}} \Xi w\right\|_{1, \ell, \tilde{\tau}}+\delta_{2} \gamma^{\alpha_{1} / 2}\left|\operatorname{tr}\left(\tilde{\tau}^{-\alpha_{1} / 2} \Xi_{1} D_{x_{d}} \Xi w\right)\right|_{0, \ell+1 / 2, \tilde{\tau}} \\
& \quad \lesssim \gamma^{\alpha_{1} / 2}\left(\left\|\tilde{\tau}^{-\alpha_{1} / 2} Q^{(2)} \Xi_{1} D_{x_{d}} \Xi w\right\|_{0, \ell, \tilde{\tau}}+\left(1-\delta_{2}\right)\left|\operatorname{tr}\left(\tilde{\tau}^{-\alpha_{1} / 2} \Xi_{1} D_{x_{d}} \Xi w\right)\right|_{0, \ell+1 / 2, \tilde{\tau}}\right)+\|w\|_{1,-M, \tilde{\tau}}
\end{aligned}
$$

and

$$
\begin{aligned}
& \gamma^{\left(\alpha_{1}+\alpha_{2}\right) / 2}\left\|\tilde{\tau}^{-\left(\alpha_{1}+\alpha_{2}\right) / 2} \Xi w\right\|_{1, \ell+1, \tilde{\tau}}+\delta_{2} \gamma^{\alpha_{1} / 2}\left|\operatorname{tr}\left(\tilde{\tau}^{-\alpha_{1} / 2} \Xi w\right)\right|_{0, \ell+3 / 2, \tilde{\tau}} \\
& \lesssim \gamma^{\alpha_{1} / 2}\left(\left\|\tilde{\tau}^{-\alpha_{1} / 2} Q^{(2)} \Xi w\right\|_{0, \ell+1, \tilde{\tau}}+\left(1-\delta_{2}\right)\left|\operatorname{tr}\left(\tilde{\tau}^{-\alpha_{1} / 2} \Xi w\right)\right|_{0, \ell+3 / 2, \tilde{\tau}}\right)+\|w\|_{0,-M, \tilde{\tau}}
\end{aligned}
$$

With (B.14)-(B.17), we achieve

$$
\begin{aligned}
& \gamma^{\left(\alpha_{1}+\alpha_{2}\right) / 2}\left\|\tilde{\tau}^{-\left(\alpha_{1}+\alpha_{2}\right) / 2} \Xi w\right\|_{2, \ell, \tilde{\tau}}+\delta_{2} \gamma^{\alpha_{1} / 2}\left|\operatorname{tr}\left(\tilde{\tau}^{-\alpha_{1} / 2} \Xi w\right)\right|_{1, \ell+1 / 2, \tilde{\tau}} \\
& \lesssim \gamma^{\alpha_{1} / 2}\left(\left\|\tilde{\tau}^{-\alpha_{1} / 2} Q^{(2)} \Xi_{1} D_{x_{d}} \Xi w\right\|_{0, \ell, \tilde{\tau}}+\left(1-\delta_{2}\right)\left|\operatorname{tr}\left(\tilde{\tau}^{-\alpha_{1} / 2} \Xi_{1} D_{x_{d}} \Xi w\right)\right|_{0, \ell+1 / 2, \tilde{\tau}}\right. \\
& \left.\quad+\left\|\tilde{\tau}^{-\alpha_{1} / 2} Q^{(2)} \Xi w\right\|_{0, \ell+1, \tilde{\tau}}+\left(1-\delta_{2}\right)\left|\operatorname{tr}\left(\tilde{\tau}^{-\alpha_{1} / 2} \Xi w\right)\right|_{0, \ell+3 / 2, \tilde{\tau}}\right)+\|w\|_{2,-M, \tilde{\tau}} .
\end{aligned}
$$

Combining this latter estimate with (B.13) we obtain

$$
\begin{aligned}
& \gamma^{\left(\alpha_{1}+\alpha_{2}\right) / 2}\left\|\tilde{\tau}^{-\left(\alpha_{1}+\alpha_{2}\right) / 2} \Xi w\right\|_{2, \ell, \tilde{\tau}}+\delta_{2} \gamma^{\alpha_{1} / 2}\left|\operatorname{tr}\left(\tilde{\tau}^{-\alpha_{1} / 2} \Xi w\right)\right|_{1, \ell+1 / 2, \tilde{\tau}} \\
& \lesssim\left\|Q^{(1)} Q^{(2)} \Xi w\right\|_{0, \ell, \tilde{\tau}}+\left(1-\delta_{1}\right)|\operatorname{tr}(\Xi w)|_{1, \ell+1 / 2, \tilde{\tau}}+\left(1-\delta_{2}\right) \gamma^{\alpha_{1} / 2}\left|\operatorname{tr}\left(\tilde{\tau}^{-\alpha_{1} / 2} \Xi w\right)\right|_{0, \ell+3 / 2, \tilde{\tau}} \\
&+\gamma^{1+\alpha_{1} / 2}\left\|\tilde{\tau}^{-\alpha_{1} / 2} \Xi w\right\|_{1, \ell, \tilde{\tau}}+\|w\|_{2,-M, \tilde{\tau}},
\end{aligned}
$$

which, with the usual semi-classical inequality (4.17)

$$
\begin{aligned}
& \gamma^{\left(\alpha_{1}+\alpha_{2}\right) / 2}\left\|\tilde{\tau}^{-\left(\alpha_{1}+\alpha_{2}\right) / 2} \Xi w\right\|_{2, \ell, \tilde{\tau}}+\delta_{2} \gamma^{\alpha_{1} / 2}\left|\operatorname{tr}\left(\tilde{\tau}^{-\alpha_{1} / 2} \Xi w\right)\right|_{1, \ell+1 / 2, \tilde{\tau}} \\
& \quad \lesssim\left\|Q^{(1)} Q^{(2)} \Xi w\right\|_{0, \ell, \tilde{\tau}}+\left(1-\delta_{1}\right)|\operatorname{tr}(\Xi w)|_{1, \ell+1 / 2, \tilde{\tau}}+\left(1-\delta_{2}\right) \gamma^{\alpha_{1} / 2}\left|\operatorname{tr}\left(\tilde{\tau}^{-\alpha_{1} / 2} \Xi w\right)\right|_{0, \ell+3 / 2, \tilde{\tau}} \\
&+\|w\|_{2,-M, \tilde{\tau}}
\end{aligned}
$$

Let us now consider two cases: 
Case $\alpha_{1}=1$ : Then $\delta_{1}=0$ and $\alpha_{2}=1$. We thus have the term $|\operatorname{tr}(\Xi w)|_{1, \ell+1 / 2, \tilde{\tau}}$ in the r.h.s. of the estimation and the sought result then holds.

Case $\alpha_{1}=0$ : Then we write

$$
\begin{aligned}
|\operatorname{tr}(\Xi w)|_{1, \ell+1 / 2, \tilde{\tau}} & \lesssim\left|\operatorname{tr}\left(Q^{(2)} \Xi w\right)\right|_{0, \ell+1 / 2, \tilde{\tau}}+|\operatorname{tr}(\Xi w)|_{0, \ell+3 / 2, \tilde{\tau}} \\
& \lesssim\left|\operatorname{tr}\left(Q^{(2)} \Xi w\right)\right|_{0, \ell+1 / 2, \tilde{\tau}}+\delta_{2}|\operatorname{tr}(\Xi w)|_{1, \ell+1 / 2, \tilde{\tau}}+\left(1-\delta_{2}\right)|\operatorname{tr}(\Xi w)|_{0, \ell+3 / 2, \tilde{\tau}} .
\end{aligned}
$$

which leads to

$$
\delta_{1}|\operatorname{tr}(\Xi w)|_{1, \ell+1 / 2, \tilde{\tau}} \lesssim \delta_{1}\left|\operatorname{tr}\left(Q^{(2)} \Xi w\right)\right|_{0, \ell+1 / 2, \tilde{\tau}}+\delta_{2}|\operatorname{tr}(\Xi w)|_{1, \ell+1 / 2, \tilde{\tau}}+\left(1-\delta_{2}\right)|\operatorname{tr}(\Xi w)|_{0, \ell+3 / 2, \tilde{\tau}} .
$$

Recalling that the term $\delta_{1}\left|\operatorname{tr}\left(Q^{(2)} \Xi w\right)\right|_{0, \ell+1 / 2, \tilde{\tau}}$ can be found in the 1.h.s. of (B.11), We thus obtain

$$
\begin{aligned}
& \gamma^{\alpha_{2} / 2}\left\|\tilde{\tau}^{-\alpha_{2} / 2} \Xi w\right\|_{2, \ell, \tilde{\tau}}+\left(\delta_{1}+\delta_{2}\right)|\operatorname{tr}(\Xi w)|_{1, \ell+1 / 2, \tilde{\tau}} \\
& \quad \lesssim\left\|Q^{(1)} Q^{(2)} \Xi w\right\|_{0, \ell, \tilde{\tau}}+\left(1-\delta_{1}\right)|\operatorname{tr}(\Xi w)|_{1, \ell+1 / 2, \tilde{\tau}}+\left(1-\delta_{2}\right)|\operatorname{tr}(\Xi w)|_{0, \ell+3 / 2, \tilde{\tau}} \\
&+\|w\|_{2,-M, \tilde{\tau}}
\end{aligned}
$$

If $\delta_{1}+\delta_{2}>0$ we then have the sought estimate in the case $\alpha_{1}=0$. If $\delta_{1}+\delta_{2}=0$ then the term $|\operatorname{tr}(\Xi w)|_{1, \ell+1 / 2, \tilde{\tau}}$ can be found in the r.h.s. of the estimation and can thus be "artificially" added in the 1.h.s..

This concludes the proof of Proposition B.7.

We now show how to obtain microlocal estimates for some products of two factors of order two.

Proposition B.8. Let assume that $Q^{-}\left(z, D_{z}, \tau, \gamma, \varepsilon\right) \in \Psi_{\tilde{\tau}}^{2,0}$ fulfills the requirement of Lemma B.1 in some conic open subset $\mathscr{U}$. Let $Q^{+}\left(z, D_{z}, \tau, \gamma, \varepsilon\right) \in \Psi_{\tilde{\tau}}^{2,0}$ be such that, there exist $\tau_{0} \geq \tau_{*}, \gamma_{0} \geq 1$ and $C>0$ such that, for $\ell \in\{0,1,2\}$ and all $\chi \in S\left(1, g_{\top}\right)$, homogeneous of degree zero, with $\operatorname{supp}(\chi) \subset \mathscr{U}$, for $\Xi=\mathrm{Op}_{\mathrm{T}}(\chi)$,

$$
\begin{aligned}
& \gamma^{\left(\alpha_{1}+\alpha_{2}\right) / 2}\left\|\tilde{\tau}^{-\left(\alpha_{1}+\alpha_{2}\right) / 2} \Xi w\right\|_{2, \ell, \tilde{\tau}}+|\operatorname{tr}(\Xi w)|_{1, \ell+1 / 2, \tilde{\tau}} \\
& \leq C\left(\left\|Q^{+} \Xi w\right\|_{0, \ell, \tilde{\tau}}+\left(1-\delta_{1}\right)|\operatorname{tr}(\Xi w)|_{1, \ell+1 / 2, \tilde{\tau}}\right. \\
&\left.+\left(1-\delta_{2}\right) \gamma^{\alpha_{1} / 2}\left|\operatorname{tr}\left(\tilde{\tau}^{-\alpha_{1} / 2} \Xi w\right)\right|_{0, \ell+3 / 2, \tilde{\tau}}+\|w\|_{2,-M, \tilde{\tau}}\right),
\end{aligned}
$$

for $\tau \geq \tau_{0}, \gamma \geq \gamma_{0}, \varepsilon \in[0,1]$, and for $w \in \mathscr{S}\left(\overline{\mathbb{R}_{+}^{N}}\right)$, where $\alpha_{1}, \alpha_{2} \in\{0,1\}$ and $\delta_{1}, \delta_{2} \in\{0,1\}$ with $\alpha_{1} \leq \alpha_{2}, 1-\delta_{1} \leq 1-\delta_{2}$ and moreover $\delta_{k}=0$ if $\alpha_{k}=1, k=1,2$. We also assume that $Q^{+} \Xi=D_{x_{d}}^{2} \Xi+T_{1,1} \Xi$ with $T_{1,1} \in \Psi_{\tilde{\tau}}^{1,1}$.

Let $M \in \mathbb{N}$ and let $\chi \in S\left(1, g_{\mathrm{T}}\right)$ be as above. In the case $\alpha_{1}+\alpha_{2}=2$, we furthermore assume that, for any $M \in \mathbb{N},\left[D_{x_{d}}+i \hat{\tau}_{\xi_{d}}, Q^{+}\right] \mathrm{Op}_{\mathrm{T}}\left(\chi_{1}\right)=(1+\varepsilon \gamma) R_{2,0} \mathrm{Op}_{\mathrm{T}}\left(\chi_{1}\right)+R_{2,-M}$, with $R_{2,0} \in \Psi_{\tilde{\tau}}^{2,0}$ and $R_{2,-M} \in \Psi_{\tilde{\tau}}^{2,-M}$, if $\chi_{1} \in S\left(1, g_{\top}\right)$ is homogeneous of degree 0 and such that $\chi_{1} \equiv 1$ in a conic neighborhood of $\operatorname{supp}(\chi)$ and $\operatorname{supp}\left(\chi_{1}\right) \subset \mathscr{U}$.

There exist $\tau_{1} \geq \tau_{*}, \gamma_{1} \geq 1, \varepsilon_{1} \in(0,1]$, and $C>0$ such that

$$
\begin{aligned}
& \gamma^{\left(\alpha_{1}+\alpha_{2}\right) / 2}\left\|\tilde{\tau}^{-\left(\alpha_{1}+\alpha_{2}\right) / 2} \Xi w\right\|_{4,0, \tilde{\tau}}+|\operatorname{tr}(\Xi w)|_{3,1 / 2, \tilde{\tau}} \\
& \leq C\left(\left\|Q^{-} Q^{+} \Xi w\right\|_{+}+\left(1-\delta_{1}\right)|\operatorname{tr}(\Xi w)|_{1,5 / 2, \tilde{\tau}}\right. \\
&\left.+\left(1-\delta_{2}\right) \gamma^{\alpha_{1} / 2}\left|\operatorname{tr}\left(\tilde{\tau}^{-\alpha_{1} / 2} \Xi w\right)\right|_{0,7 / 2, \tilde{\tau}}+\|w\|_{4,-M, \tilde{\tau}}\right),
\end{aligned}
$$

for $\tau \geq \tau_{1}, \gamma \geq \gamma_{1}, \varepsilon \in\left[0, \varepsilon_{1}\right]$, and for $w \in \mathscr{S}\left(\overline{\mathbb{R}_{+}^{N}}\right)$. In the case $\alpha_{1}+\alpha_{2} \leq 1$, we can take $\varepsilon_{1}=1$. 
In Section 4 , for example, this proposition will be applied to $Q^{+}=Q_{1,+} Q_{2,+}$ for which an estimation of the form of (B.18) will hold by Proposition B.7. Note that this proposition, in the case $\alpha_{1}+\alpha_{2}=2$, is one instance where it is important to take $\varepsilon>0$ sufficiently small.

Proof. We introduce $\chi_{1} \in S\left(1, g_{\mathrm{T}}\right)$ that is such that $\chi_{1} \equiv 1$ on $\operatorname{supp}(\chi)$ and $\operatorname{supp}\left(\chi_{1}\right) \subset \mathscr{U}$. For concision, we write $\Xi=\mathrm{Op}_{\mathrm{T}}(\chi)$ and $\Xi_{1}=\mathrm{Op}_{\mathrm{T}}\left(\chi_{1}\right)$. Here, $M$ will denote an arbitrary large integer whose value may change from one line to the other.

Using $Q^{+} \Xi w$ as the unknown function in the estimate of Lemma B.1 for the operator $Q^{-}$:

$$
\begin{aligned}
\left\|Q^{+} \Xi w\right\|_{2,0, \tilde{\tau}}+\left|\operatorname{tr}\left(Q^{+} \Xi w\right)\right|_{1,1 / 2, \tilde{\tau}} \\
\quad \lesssim\left\|\Xi_{1} Q^{+} \Xi w\right\|_{2,0, \tilde{\tau}}+\left|\operatorname{tr}\left(\Xi_{1} Q^{+} \Xi w\right)\right|_{1,1 / 2, \tilde{\tau}}+\|w\|_{4,-M, \tilde{\tau}} \\
\quad \lesssim\left\|Q^{-} \Xi_{1} Q^{+} \Xi w\right\|_{+}+\|w\|_{4,-M, \tilde{\tau}} \\
\quad \lesssim\left\|Q^{-} Q^{+} \Xi w\right\|_{+}+\|w\|_{4,-M, \tilde{\tau}} .
\end{aligned}
$$

Combining (B.18), for $\ell=2$, with (B.20) we find

$$
\begin{aligned}
& \left\|Q^{+} \Xi w\right\|_{2,0, \tilde{\tau}}+\left|\operatorname{tr}\left(Q^{+} \Xi w\right)\right|_{1,1 / 2, \tilde{\tau}}+|\operatorname{tr}(\Xi w)|_{1,5 / 2, \tilde{\tau}} \\
& \quad \lesssim \\
& \quad+\left\|Q^{-} Q^{+} \Xi w\right\|_{+}+\left(1-\delta_{1}\right)|\operatorname{tr}(\Xi w)|_{1,5 / 2, \tilde{\tau}}+\left(1-\delta_{2}\right) \gamma^{\alpha_{1} / 2}\left|\operatorname{tr}\left(\tilde{\tau}^{-\alpha_{1} / 2} \Xi w\right)\right|_{0,7 / 2, \tilde{\tau}} \\
& \quad+\|w\|_{4,-M, \tilde{\tau} .}
\end{aligned}
$$

We now make the following claim whose proof is given below.

Lemma B.9. There exists $C>0$ such that

$$
|\operatorname{tr}(\Xi v)|_{3,1 / 2, \tilde{\tau}} \leq C\left(\left|\operatorname{tr}\left(Q^{+} \Xi w\right)\right|_{1,1 / 2, \tilde{\tau}}+|\operatorname{tr}(\Xi v)|_{1,5 / 2, \tilde{\tau}}\right) .
$$

This gives

$$
\begin{aligned}
\left\|Q^{+} \Xi w\right\|_{2,0, \tilde{\tau}}+|\operatorname{tr}(\Xi v)|_{3,1 / 2, \tilde{\tau}} \lesssim\left\|Q^{-} Q^{+} \Xi w\right\|_{+}+\left(1-\delta_{1}\right)|\operatorname{tr}(\Xi w)|_{1,5 / 2, \tilde{\tau}} \\
+\left(1-\delta_{2}\right) \gamma^{\alpha_{1} / 2}\left|\operatorname{tr}\left(\tilde{\tau}^{-\alpha_{1} / 2} \Xi w\right)\right|_{0,7 / 2, \tilde{\tau}}+\|w\|_{4,-M, \tilde{\tau}} .
\end{aligned}
$$

First, we treat the case $\alpha_{1}+\alpha_{2} \leq 1$. As $\alpha_{1} \leq \alpha_{2}$ then $\alpha_{1}=0$. We write

$$
\begin{aligned}
& \sum_{j=0}^{2}\left(\left\|Q^{+} \Xi_{1} D_{x_{d}}^{j} \Xi w\right\|_{0,2-j, \tilde{\tau}}+\left|\operatorname{tr}\left(\Xi_{1} D_{x_{d}}^{j} \Xi v\right)\right|_{1,5 / 2-j, \tilde{\tau}}\right) \\
& \quad \lesssim \sum_{j=0}^{2}\left(\left\|Q^{+} D_{x_{d}}^{j} \Xi w\right\|_{0,2-j, \tilde{\tau}}+\left|\operatorname{tr}\left(D_{x_{d}}^{j} \Xi v\right)\right|_{1,5 / 2-j, \tilde{\tau}}\right)+\|w\|_{4,-M, \tilde{\tau}} \\
& \quad \lesssim \sum_{j=0}^{2}\left(\left\|D_{x_{d}}^{j} Q^{+} \Xi w\right\|_{0,2-j, \tilde{\tau}}+\left|\operatorname{tr}\left(D_{x_{d}}^{j} \Xi v\right)\right|_{1,5 / 2-j, \tilde{\tau}}\right)+\gamma\|\Xi w\|_{3,0}+\|w\|_{4,-M, \tilde{\tau}} \\
& \quad \lesssim\left\|Q^{+} \Xi w\right\|_{2,0, \tilde{\tau}}+|\operatorname{tr}(\Xi v)|_{3,1 / 2, \tilde{\tau}}+\gamma\|\Xi w\|_{3,0}+\|w\|_{4,-M, \tilde{\tau}} .
\end{aligned}
$$

With (B.22) we then find

$$
\begin{aligned}
\sum_{j=0}^{2}( & \left.\left\|Q^{+} \Xi_{1} D_{x_{d}}^{j} \Xi w\right\|_{0,2-j, \tilde{\tau}}+\left|\operatorname{tr}\left(\Xi_{1} D_{x_{d}}^{j} \Xi v\right)\right|_{1,5 / 2-j, \tilde{\tau}}\right) \\
\lesssim & \left\|Q^{-} Q^{+} \Xi w\right\|_{+}+\left(1-\delta_{1}\right)|\operatorname{tr}(\Xi w)|_{1,5 / 2, \tilde{\tau}}+\left(1-\delta_{2}\right)|\operatorname{tr}(\Xi w)|_{0,7 / 2, \tilde{\tau}} \\
& +\gamma\|\Xi w\|_{3,0}+\|w\|_{4,-M, \tilde{\tau}} .
\end{aligned}
$$


Now, applying (B.18) with $\ell=2-j$, we obtain

$$
\begin{aligned}
& \sum_{j=0}^{2}\left(\gamma^{\alpha_{2} / 2}\left\|\tilde{\tau}^{-\alpha_{2} / 2} \Xi_{1} D_{x_{d}}^{j} \Xi w\right\|_{2,2-j, \tilde{\tau}}+\left|\operatorname{tr}\left(\Xi_{1} D_{x_{d}}^{j} \Xi w\right)\right|_{1,5 / 2-j, \tilde{\tau}}\right) \\
& \lesssim \\
& \quad\left\|Q^{-} Q^{+} \Xi w\right\|_{+}+\left(1-\delta_{1}\right)|\operatorname{tr}(\Xi w)|_{1,5 / 2, \tilde{\tau}}+\left(1-\delta_{2}\right)|\operatorname{tr}(\Xi w)|_{0,7 / 2, \tilde{\tau}} \\
& \quad+\gamma\|\Xi w\|_{3,0}+\|w\|_{4,-M, \tilde{\tau}} .
\end{aligned}
$$

With Lemma 4.14, we write, for $\tau$ chosen sufficiently large,

$$
\begin{aligned}
\gamma^{\alpha_{2} / 2}\left\|\tilde{\tau}^{-\alpha_{2} / 2} \Xi w\right\|_{4,0, \tilde{\tau}}+|\operatorname{tr}(\Xi w)|_{3,1 / 2, \tilde{\tau}} \\
\quad \asymp \sum_{j=0}^{2}\left(\gamma^{\alpha_{2} / 2}\left\|\tilde{\tau}^{-\alpha_{2} / 2} D_{x_{d}}^{j} \Xi w\right\|_{2,2-j, \tilde{\tau}}+\left|\operatorname{tr}\left(D_{x_{d}}^{j} \Xi w\right)\right|_{1,5 / 2-j, \tilde{\tau}}\right) \\
\quad \lesssim \sum_{j=0}^{2}\left(\gamma^{\alpha_{2} / 2}\left\|\tilde{\tau}^{-\alpha_{2} / 2} \Xi_{1} D_{x_{d}}^{j} \Xi w\right\|_{2,2-j, \tilde{\tau}}+\left|\operatorname{tr}\left(\Xi_{1} D_{x_{d}}^{j} \Xi w\right)\right|_{1,5 / 2-j, \tilde{\tau}}\right)+\|w\|_{4,-M, \tilde{\tau}} .
\end{aligned}
$$

Finally, using (B.23) we obtain

$$
\begin{aligned}
\gamma^{\alpha_{2} / 2}\left\|\tilde{\tau}^{-\alpha_{2} / 2} \Xi w\right\|_{4,0, \tilde{\tau}}+|\operatorname{tr}(\Xi w)|_{3,1 / 2, \tilde{\tau}} \\
\lesssim \\
\quad \\
\quad+\gamma Q^{-} Q^{+} \Xi w\left\|_{+}+\left(1-\delta_{1}\right)|\operatorname{tr}(\Xi w)|_{1,5 / 2, \tilde{\tau}}+\left(1-\delta_{2}\right)|\operatorname{tr}(\Xi w)|_{0,7 / 2, \tilde{\tau}}+\right\| w \|_{4,-M, \tilde{\tau}},
\end{aligned}
$$

and taking $\tau$ sufficiently large, as $0 \leq \alpha_{2} \leq 1$, we achieve the sought estimate.

Second, we treat the case $\alpha_{1}+\alpha_{2}=2$, that is, $\alpha_{1}=\alpha_{2}=1$. We set $\tilde{D}_{x_{d}}=D_{x_{d}}+i \hat{\tau}_{\xi_{d}} \in \Psi_{\tilde{\tau}}^{1,0}$. We use the further assumption made in this case, namely, for any $M \in \mathbb{N},\left[\tilde{D}_{x_{d}}, Q^{+}\right] \Xi_{1}=(1+$ $\varepsilon \gamma) R_{2,0} \Xi_{1}+R_{2,-M}$ with $R_{2,0} \in \Psi_{\tilde{\tau}}^{2,0}$ and $R_{2,-M} \in \Psi_{\tilde{\tau}}^{2,-M}$. We write

$$
\begin{aligned}
\sum_{j=0}^{2}\left(\left\|Q^{+} \Xi_{1} \tilde{D}_{x_{d}}^{j} \Xi w\right\|_{0,2-j, \tilde{\tau}}+\left|\operatorname{tr}\left(\Xi_{1} \tilde{D}_{x_{d}}^{j} \Xi v\right)\right|_{1,5 / 2-j, \tilde{\tau}}\right) \\
\quad \lesssim \sum_{j=0}^{2}\left(\left\|Q^{+} \tilde{D}_{x_{d}}^{j} \Xi_{1} \Xi w\right\|_{0,2-j, \tilde{\tau}}+\left|\operatorname{tr}\left(\tilde{D}_{x_{d}}^{j} \Xi v\right)\right|_{1,5 / 2-j, \tilde{\tau}}\right)+\|w\|_{4,-M, \tilde{\tau}} \\
\quad \lesssim \sum_{j=0}^{2}\left(\left\|\tilde{D}_{x_{d}}^{j} Q^{+} \Xi_{1} \Xi w\right\|_{0,2-j, \tilde{\tau}}+\left|\operatorname{tr}\left(\tilde{D}_{x_{d}}^{j} \Xi v\right)\right|_{1,5 / 2-j, \tilde{\tau}}\right)+(1+\varepsilon \gamma)\|\Xi w\|_{3,0}+\|w\|_{4,-M, \tilde{\tau}} \\
\quad \lesssim\left\|Q^{+} \Xi w\right\|_{2,0, \tilde{\tau}}+|\operatorname{tr}(\Xi v)|_{3,1 / 2, \tilde{\tau}}+(1+\varepsilon \gamma)\|\Xi w\|_{3,0}+\|w\|_{4,-M, \tilde{\tau}} .
\end{aligned}
$$

With (B.22) we then find

$$
\begin{aligned}
\sum_{j=0}^{2}( & \left.\left\|Q^{+} \Xi_{1} \tilde{D}_{x_{d}}^{j} \Xi w\right\|_{0,2-j, \tilde{\tau}}+\left|\operatorname{tr}\left(\Xi_{1} \tilde{D}_{x_{d}}^{j} \Xi v\right)\right|_{1,5 / 2-j, \tilde{\tau}}\right) \\
& \leq\left\|Q^{-} Q^{+} \Xi w\right\|_{+}+\left(1-\delta_{1}\right)|\operatorname{tr}(\Xi w)|_{1,5 / 2, \tilde{\tau}}+\left(1-\delta_{2}\right) \gamma^{1 / 2}\left|\tilde{\tau}^{-1 / 2} \operatorname{tr}(\Xi w)\right|_{0,7 / 2, \tilde{\tau}} \\
& +(1+\varepsilon \gamma)\|\Xi w\|_{3,0}+\|w\|_{4,-M, \tilde{\tau}} .
\end{aligned}
$$

Now, applying (B.18) with $\ell=2-j$, we obtain

$$
\begin{aligned}
& \sum_{j=0}^{2}\left(\gamma\left\|\tilde{\tau}^{-1} \Xi_{1} \tilde{D}_{x_{d}}^{j} \Xi w\right\|_{2,2-j, \tilde{\tau}}+\left|\operatorname{tr}\left(\Xi_{1} \tilde{D}_{x_{d}}^{j} \Xi w\right)\right|_{1,5 / 2-j, \tilde{\tau}}\right) \\
& \quad \lesssim\left\|Q^{-} Q^{+} \Xi w\right\|_{+}+\left(1-\delta_{1}\right)|\operatorname{tr}(\Xi w)|_{1,5 / 2, \tilde{\tau}}+\left(1-\delta_{2}\right) \gamma^{1 / 2}\left|\tilde{\tau}^{-1 / 2} \operatorname{tr}(\Xi w)\right|_{0,7 / 2, \tilde{\tau}} \\
&+(1+\varepsilon \gamma)\|\Xi w\|_{3,0}+\|w\|_{4,-M, \tilde{\tau}} .
\end{aligned}
$$


Now, as $\left[\tilde{D}_{x_{d}}, \tilde{\tau}^{-1}\right] \in \gamma \Psi_{\tilde{\tau}}^{0,-1}$, we have

$$
\begin{aligned}
& \sum_{j=0}^{2}\left(\gamma\left\|\tilde{D}_{x_{d}}^{j} \tilde{\tau}^{-1} \Xi w\right\|_{2,2-j, \tilde{\tau}}+\left|\operatorname{tr}\left(\tilde{D}_{x_{d}}^{j} \Xi w\right)\right|_{1,5 / 2-j \tilde{\tau}}\right) \\
& \lesssim \sum_{j=0}^{2}\left(\gamma\left\|\tilde{\tau}^{-1} \tilde{D}_{x_{d}}^{j} \Xi w\right\|_{2,2-j, \tilde{\tau}}+\left|\operatorname{tr}\left(\tilde{D}_{x_{d}}^{j} \Xi w\right)\right|_{1,5 / 2-j, \tilde{\tau}}\right)+\gamma^{2}\left\|\tilde{\tau}^{-1} \Xi w\right\|_{3,0} \\
& \lesssim \sum_{j=0}^{2}\left(\gamma\left\|\tilde{\tau}^{-1} \Xi_{1} \tilde{D}_{x_{d}}^{j} \Xi w\right\|_{2,2-j, \tilde{\tau}}+\left|\operatorname{tr}\left(\Xi_{1} \tilde{D}_{x_{d}}^{j} \Xi w\right)\right|_{1,5 / 2-j, \tilde{\tau}}\right) \\
& \quad+\gamma^{2}\left\|\tilde{\tau}^{-1} \Xi w\right\|_{3,0}+\|w\|_{4,-M, \tilde{\tau}},
\end{aligned}
$$

yielding with (B.24), as $\gamma^{2} \tilde{\tau}^{-1} \lesssim 1$,

$$
\begin{aligned}
& \sum_{j=0}^{2}\left(\gamma\left\|\tilde{D}_{x_{d}}^{j} \tilde{\tau}^{-1} \Xi w\right\|_{2,2-j, \tilde{\tau}}+\left|\operatorname{tr}\left(\tilde{D}_{x_{d}}^{j} \Xi w\right)\right|_{1,5 / 2-j, \tilde{\tau}}\right) \\
& \lesssim\left\|Q^{-} Q^{+} \Xi w\right\|_{+}+\left(1-\delta_{1}\right)|\operatorname{tr}(\Xi w)|_{1,5 / 2, \tilde{\tau}}+\left(1-\delta_{2}\right) \gamma^{1 / 2}\left|\tilde{\tau}^{-1 / 2} \operatorname{tr}(\Xi w)\right|_{0,7 / 2, \tilde{\tau}} \\
&+(1+\varepsilon \gamma)\|\Xi w\|_{3,0}+\|w\|_{4,-M, \tilde{\tau}} .
\end{aligned}
$$

As $D_{x_{d}}-\tilde{D}_{x_{d}}=T \in \Psi_{\tilde{\tau}}^{0,1}$, observe that we have

$$
\left\|D_{x_{d}} \tilde{\tau}^{-1} \Xi w\right\|_{2,1, \tilde{\tau}} \lesssim\left\|\tilde{D}_{x_{d}} \tilde{\tau}^{-1} \Xi w\right\|_{2,1, \tilde{\tau}}+\left\|\tilde{\tau}^{-1} \Xi w\right\|_{2,2, \tilde{\tau}},
$$

meaning that we have

$$
\left\|\tilde{\tau}^{-1} \Xi w\right\|_{3,1, \tilde{\tau}} \lesssim\left\|\tilde{D}_{x_{d}} \tilde{\tau}^{-1} \Xi w\right\|_{2,1, \tilde{\tau}}+\left\|\tilde{\tau}^{-1} \Xi w\right\|_{2,2, \tilde{\tau}} .
$$

Next, we write

$$
\begin{aligned}
\left\|D_{x_{d}}^{2} \tilde{\tau}^{-1} \Xi w\right\|_{2,0, \tilde{\tau}} & \lesssim\left\|D_{x_{d}} \tilde{D}_{x_{d}} \tilde{\tau}^{-1} \Xi w\right\|_{2,0, \tilde{\tau}}+\left\|D_{x_{d}} T \tilde{\tau}^{-1} \Xi w\right\|_{2,0, \tilde{\tau}} \\
& \lesssim\left\|\tilde{D}_{x_{d}}^{2} \tilde{\tau}^{-1} \Xi w\right\|_{2,0, \tilde{\tau}}+\left\|\tilde{D}_{x_{d}} \tilde{\tau}^{-1} \Xi w\right\|_{2,1, \tilde{\tau}}+\left\|\tilde{\tau}^{-1} \Xi w\right\|_{3,1, \tilde{\tau}}
\end{aligned}
$$

and thus

$$
\left\|\tilde{\tau}^{-1} \Xi w\right\|_{4,0, \tilde{\tau}} \lesssim \sum_{j=0}^{2}\left\|\tilde{D}_{x_{d}}^{j} \tilde{\tau}^{-1} \Xi w\right\|_{2,2-j, \tilde{\tau}}
$$

Similarly, we find

$$
|\operatorname{tr}(\Xi w)|_{3,1 / 2, \tilde{\tau}} \lesssim \sum_{j=0}^{2}\left|\operatorname{tr}\left(\tilde{D}_{x_{d}}^{j} \Xi w\right)\right|_{1,5 / 2-j, \tilde{\tau}} \cdot
$$

With (B.25) we thus obtain

$$
\begin{aligned}
& \gamma\left\|\tilde{\tau}^{-1} \Xi w\right\|_{4,0, \tilde{\tau}}+|\operatorname{tr}(\Xi w)|_{3,1 / 2, \tilde{\tau}} \\
& \quad \lesssim\left\|Q^{-} Q^{+} \Xi w\right\|_{+}+\left(1-\delta_{1}\right)|\operatorname{tr}(\Xi w)|_{1,5 / 2, \tilde{\tau}}+\left(1-\delta_{2}\right) \gamma^{1 / 2}\left|\tilde{\tau}^{-1 / 2} \operatorname{tr}(\Xi w)\right|_{0,7 / 2, \tilde{\tau}} \\
&+(1+\varepsilon \gamma)\|\Xi w\|_{3,0}+\|w\|_{4,-M, \tilde{\tau}}
\end{aligned}
$$

Then, taking $\gamma$ sufficiently large and $\varepsilon>0$ sufficiently small we obtain the sought estimate.

Proof of Lemma B.9. Recalling that $Q^{+} \Xi=D_{x_{d}}^{2} \Xi+T_{1,1} \Xi$, where $T_{1,1} \in \Psi_{\tilde{\tau}}^{1,1}$, we have

$$
\begin{aligned}
|\operatorname{tr}(\Xi v)|_{2,3 / 2, \tilde{\tau}} & \asymp\left|\operatorname{tr}\left(D_{x_{d}}^{2} \Xi v\right)\right|_{0,3 / 2, \tilde{\tau}}+|\operatorname{tr}(\Xi v)|_{1,5 / 2, \tilde{\tau}}=\left|\operatorname{tr}\left(\left(Q^{+}-T_{1,1}\right) \Xi v\right)\right|_{0,3 / 2, \tilde{\tau}}+|\operatorname{tr}(\Xi v)|_{1,5 / 2, \tilde{\tau}} \\
& \lesssim\left|\operatorname{tr}\left(Q^{+} \Xi v\right)\right|_{0,3 / 2, \tilde{\tau}}+|\operatorname{tr}(\Xi v)|_{1,5 / 2, \tilde{\tau}} .
\end{aligned}
$$


We then write

$$
\begin{aligned}
|\operatorname{tr}(\Xi v)|_{3,1 / 2, \tilde{\tau}} & \asymp\left|\operatorname{tr}\left(D_{x_{d}}^{3} \Xi v\right)\right|_{0,1 / 2, \tilde{\tau}}+|\operatorname{tr}(\Xi v)|_{2,3 / 2, \tilde{\tau}}=\left|\operatorname{tr}\left(D_{x_{d}}\left(Q^{+}-T_{1,1}\right) \Xi v\right)\right|_{0,1 / 2, \tilde{\tau}}+|\operatorname{tr}(\Xi v)|_{2,3 / 2, \tilde{\tau}} \\
& \lesssim\left|\operatorname{tr}\left(Q^{+} \Xi v\right)\right|_{1,1 / 2, \tilde{\tau}}+|\operatorname{tr}(\Xi v)|_{2,3 / 2, \tilde{\tau}} .
\end{aligned}
$$

Combining the two estimates yields the result.

B.5. An Estimate for $Q_{k}$. We recall that

$$
Q_{k}=\left(D_{x_{d}}+i \hat{\tau}_{\xi_{d}}\left(\varrho^{\prime}\right)\right)^{2}+(-1)^{k} i\left(D_{s}+i \hat{\tau}_{\sigma}\left(\varrho^{\prime}\right)\right)^{2}+r\left(x, D_{x^{\prime}}+i \hat{\tau}_{\xi^{\prime}}\left(\varrho^{\prime}\right)\right),
$$

with $k=1,2$. For this operator we have the following estimation.

Proposition B.10. Let $V^{\prime} \Subset V$. Let $\ell \in \mathbb{R}$. There exist $\tau_{0} \geq \tau_{*}, \gamma_{0} \geq 1$ and $C>0$ such that

$$
\gamma^{1 / 2}\left\|\tilde{\tau}^{-1 / 2} v\right\|_{2, \ell, \tilde{\tau}}+|\operatorname{tr}(v)|_{1, \ell+1 / 2, \tilde{\tau}} \leq C\left(\left\|Q_{k} v\right\|_{0, \ell, \tilde{\tau}}+|\operatorname{tr}(v)|_{0, \ell+3 / 2, \tilde{\tau}}\right), \quad k=1,2,
$$

for $\tau \geq \tau_{0}, \gamma \geq \gamma_{0}, \varepsilon \in[0,1]$, and for $v=\left.w\right|_{\mathbb{R}_{+}^{N}}$, with $w \in \mathscr{C}_{c}^{\infty}\left(\mathbb{R}^{N}\right)$ and $\operatorname{supp}(w) \subset V^{\prime}$.

The open neighborhood $V$ is that introduced in Section 4.2.

Proof. Let $k$ be equal to 1 or 2 . We write $Q$ in place of $Q_{k}$ for concision. We also write $\mu$ in place of $\mu_{k}$.

We need to define microlocalization symbols and operators as in Section 4.4 and use some of the symbols introduced therein. Let $\chi_{V^{\prime}} \in \mathscr{C}^{\infty}\left(\mathbb{R}^{N}\right)$ be such that $\operatorname{supp}\left(\chi_{V^{\prime}}\right) \subset V$ and $\chi_{V^{\prime}} \equiv 1$ on an open neighborhood of $V^{\prime}$.

For $\delta \in(0,1]$, we set

$$
\chi_{\delta,-}\left(\varrho^{\prime}\right)=\chi_{V^{\prime}}(z) \chi_{-}\left(\mu\left(\varrho^{\prime}\right) / \delta\right) \in S\left(1, g_{\top}\right) \quad \tilde{\chi}_{\delta, 0}\left(\varrho^{\prime}\right)=\chi_{V^{\prime}}(z)\left(1-\chi_{-}\left(\mu\left(\varrho^{\prime}\right) / \delta\right)\right) \in S\left(1, g_{\top}\right),
$$

for $\chi_{-}$defined in Section 4.4, and observe that $\chi_{\delta,-}+\chi_{\delta, 0}=1$ on $\mathcal{M}_{\mathrm{T}, V^{\prime}}$. We set $\Xi_{\delta,-}=\operatorname{Op}_{\mathrm{T}}\left(\chi_{\delta,-}\right)$ and $\Xi_{\delta, 0}=\mathrm{Op}_{\mathrm{T}}\left(\tilde{\chi}_{\delta, 0}\right)$.

In a conic neighborhood of $\operatorname{supp}\left(\chi_{\delta,-}\right) \subset \mathcal{M}_{\mathrm{T}, V}$ we have $\mu \leq-C \delta$. As (4.20) holds in $V$ we have $\hat{\tau}_{\xi_{d}} \geq C \tilde{\tau}$ and thus $\left|\hat{\tau}_{\xi}\right| \asymp \tilde{\tau}$. Thus, by Lemma 4.18, both roots of the symbol $q$ of the operator $Q$ are in the lower half complex plane. Then, with Lemma B.1 we have the following perfect elliptic estimate, for any $M>0$,

$$
\left\|\Xi_{\delta,-} v\right\|_{2,0, \tilde{\tau}}+\left|\operatorname{tr}\left(\Xi_{\delta,-} v\right)\right|_{1,1 / 2, \tilde{\tau}} \lesssim\left\|Q \Xi_{\delta,-} v\right\|_{+}+\|v\|_{2,-M, \tilde{\tau}}
$$

for $v \in \mathscr{S}\left(\overline{\mathbb{R}_{+}^{N}}\right)$, for $\tau \geq \tau_{*}, \gamma \geq 1$ chosen sufficiently large, and $\varepsilon \in[0,1]$.

We now let $\underline{\chi}_{\delta}, \chi_{\delta, 1} \in S\left(1, g_{\mathrm{T}}\right)$ supported in $\mathcal{M}_{\mathrm{T}, V}$, homogeneous of degree zero, be such that $\mu \geq-C \delta$ on their supports and $\chi_{\delta, 1} \equiv 1$ in a conic neighborhood of $\operatorname{supp}\left(\tilde{\chi}_{\delta, 0}\right)$ and $\underline{\chi}_{\delta} \equiv 1$ in a conic neighborhood of $\operatorname{supp}\left(\chi_{\delta, 1}\right)$.

We choose $\delta>0$ sufficiently small so that the result of Lemma 4.22 applies, that is, on $\operatorname{supp}\left(\underline{\chi}_{\delta}\right)$ the roots of $q$ are simple. We have

$$
q(\varrho)=q_{-}(\varrho) q_{+}(\varrho), \quad q_{ \pm}(\varrho)=\xi_{d}-\rho_{ \pm}\left(\varrho^{\prime}\right) .
$$

We set $Q_{ \pm}:=D_{x_{d}}-\mathrm{Op}_{\mathrm{T}}{ }^{w}\left(\underline{\chi}^{2} \rho_{ \pm}\right)$.

We shall denote by $R_{j, k}$ as a generic operator in $\Psi_{\tilde{\tau}}^{j, k}, j \in \mathbb{N}, k \in \mathbb{R}$, whose expression may change from one line to the other. We denote by $M$ an arbitrary large integer whose value may change from one line to the other. We have with a proof similar to that of Lemma 4.33,

$$
Q \Xi_{\delta, 0}=Q_{-} Q_{+} \Xi_{\delta, 0}+\gamma R_{1,0} \Xi_{\delta, 0}+R_{2,-M} .
$$


In a conic neighborhood of $\operatorname{supp}\left(\tilde{\chi}_{\delta, 0}\right)$, the root of the symbol of $Q_{-}$is in the lower half complex plane. Then, with Lemma B.1, we have the following perfect elliptic estimate, for any $M>0$,

$$
\left\|\Xi_{\delta, 0} v\right\|_{1,0, \tilde{\tau}}+\left|\operatorname{tr}\left(\Xi_{\delta, 0} v\right)\right|_{0,1 / 2, \tilde{\tau}} \lesssim\left\|Q_{-} \Xi_{\delta, 0} v\right\|_{+}+\|v\|_{1,-M, \tilde{\tau}}
$$

for $v \in \mathscr{S}\left(\overline{\mathbb{R}_{+}^{N}}\right)$, for $\tau \geq \tau_{*}, \gamma \geq 1$ chosen sufficiently large, and $\varepsilon \in[0,1]$.

For $Q_{+}$we have the following estimate, characterized by the loss of a half derivative and a trace observation, as given by Lemma B.6,

$$
\gamma^{1 / 2}\left\|\tilde{\tau}^{m-1 / 2} \Xi_{\delta, 0} v\right\|_{1, \ell, \tilde{\tau}} \lesssim\left\|\tilde{\tau}^{m} Q_{+} \Xi_{\delta, 0} v\right\|_{0, \ell, \tilde{\tau}}+\left|\operatorname{tr}\left(\tilde{\tau}^{m} \Xi_{\delta, 0} v\right)\right|_{0, \ell+1 / 2, \tilde{\tau}}+\|v\|_{0,-M, \tilde{\tau}},
$$

for $v \in \mathscr{S}\left(\overline{\mathbb{R}_{+}^{N}}\right)$ and $\ell \in \mathbb{R}$, and for $\tau$ and $\gamma$ chosen sufficiently large, and $\varepsilon \in[0,1]$. Then, according to Proposition B.7, applied with $\alpha_{1}=0, \alpha_{2}=1, \delta_{1}=1$, and $\delta_{2}=0$, we have the following estimates for the operator $Q_{-} Q_{+}$, for $M>0$ and $\ell \in \mathbb{R}$,

$$
\gamma^{1 / 2}\left\|\tilde{\tau}^{-1 / 2} \Xi_{\delta, 0} v\right\|_{2,0, \tilde{\tau}}+\left|\operatorname{tr}\left(\Xi_{\delta, 0} v\right)\right|_{1,1 / 2, \tilde{\tau}} \lesssim\left\|Q_{-} Q_{+} \Xi_{\delta, 0} v\right\|_{+}+\left|\operatorname{tr}\left(\Xi_{\delta, 0} v\right)\right|_{0,3 / 2, \tilde{\tau}}+\|v\|_{2,-M, \tilde{\tau}},
$$

for $v \in \mathscr{S}\left(\overline{\mathbb{R}_{+}^{N}}\right)$, and for $\tau$ and $\gamma$ chosen sufficiently large. With (B.27) we thus obtain

$$
\gamma^{1 / 2}\left\|\tilde{\tau}^{-1 / 2} \Xi_{\delta, 0} v\right\|_{2,0, \tilde{\tau}}+\left|\operatorname{tr}\left(\Xi_{\delta, 0} v\right)\right|_{1,1 / 2, \tilde{\tau}} \lesssim\left\|Q \Xi_{\delta, 0} v\right\|_{+}+\left|\operatorname{tr}\left(\Xi_{\delta, 0} v\right)\right|_{0,3 / 2, \tilde{\tau}}+\|v\|_{2,-M, \tilde{\tau}},
$$

for $\tau$ chosen sufficiently large with the usual semi-classical inequality (4.17).

Using that $\chi_{\delta,-}+\chi_{\delta, 0}=1$ on $\mathcal{M}_{\mathrm{T}, V^{\prime}}$ we obtain, with (B.26) and (B.29)

$$
\begin{aligned}
\gamma^{1 / 2}\left\|\tilde{\tau}^{-1 / 2} v\right\|_{2,0, \tilde{\tau}}+|\operatorname{tr}(v)|_{1,1 / 2, \tilde{\tau}} & \\
& \lesssim \gamma^{1 / 2}\left\|\tilde{\tau}^{-1 / 2} \Xi_{\delta,-} v\right\|_{2,0, \tilde{\tau}}+\gamma^{1 / 2}\left\|\tilde{\tau}^{-1 / 2} \Xi_{\delta, 0} v\right\|_{2,0, \tilde{\tau}}+\left|\operatorname{tr}\left(\Xi_{\delta,-} v\right)\right|_{1,1 / 2, \tilde{\tau}}+\left|\operatorname{tr}\left(\Xi_{\delta, 0} v\right)\right|_{1,1 / 2, \tilde{\tau}} \\
& \lesssim\left\|Q \Xi_{\delta,-} v\right\|_{+}+\left\|Q \Xi_{\delta, 0} v\right\|_{+}+\left|\operatorname{tr}\left(\Xi_{\delta, 0} v\right)\right|_{0,3 / 2, \tilde{\tau}}+\|v\|_{2,-M, \tilde{\tau}},
\end{aligned}
$$

for $v=\left.w\right|_{\mathbb{R}_{+}^{N}}$, with $w \in \mathscr{C}_{c}^{\infty}\left(\mathbb{R}^{N}\right)$ and $\operatorname{supp}(w) \subset V^{\prime}$. Observing that $\left[Q, \Xi_{\delta,-}\right]$ and $\left[Q, \Xi_{\delta, 0}\right]$ are both in $\gamma \Psi_{\tilde{\tau}}^{1,0}$ we conclude the proof with the usual semi-classical inequality (4.17) for $\tau$ chosen sufficiently large.

Conflict of interest: The authors declare that they have no conflict of interest.

\section{REFERENCES}

[AB80] S. Alinhac and M. S. Baouendi, Uniqueness for the characteristic Cauchy problem and strong unique continuation for higher order partial differential inequalities, Amer. J. Math. 102 (1980), no. 1, 179-217.

[AB06] Fatiha Alabau-Boussouira, Piecewise multiplier method and nonlinear integral inequalities for Petrowsky equation with nonlinear dissipation, J. Evol. Equ. 6 (2006), no. 1, 95-112.

[ABA11] F. Alabau-Boussouira and K. Ammari, Sharp energy estimates for nonlinearly locally damped PDEs via observability for the associated undamped system, J. Funct. Anal. 260 (2011), no. 8, 2424-2450.

[ABPT17] F. Alabau-Boussouira, Y. Privat, and E. Trélat, Nonlinear damped partial differential equations and their uniform discretizations, J. Funct. Anal. 273 (2017), no. 1, 352-403.

[AE13] J. Apraiz and L. Escauriaza, Null-control and measurable sets, ESAIM Control Optim. Calc. Var. 19 (2013), 239-254.

[Ali80] S. Alinhac, Non-unicité pour des opérateurs différentiels à caractéristiques complexes simples, Ann. Sci. École Norm. Sup. (4) 13 (1980), no. 3, 385-393.

[ATT07] K. Ammari, M. Tucsnak, and G. Tenenbaum, A sharp geometric condition for the boundary exponential stabilizability of a square plate by moment feedbacks only, Control of coupled partial differential equations, Internat. Ser. Numer. Math., vol. 155, Birkhäuser, Basel, 2007, pp. 1-11. 
[Bar00] V. Barbu, Exact controllability of the superlinear heat equation, Appl. Math. Optim. 42 (2000), 73-89.

[BD08] C. J. K. Batty and T. Duyckaerts, Non-uniform stability for bounded semi-groups on Banach spaces, J. Evol. Equ. (2008), 765-780.

[Bel03] M. Bellassoued, Carleman estimates and distribution of resonances for the transparent obstacle and application to the stabilization, Asymptotic Anal. 35 (2003), 257-279.

[BK81] A. L. Bukhgeim and M. V. Klibanov, Global uniqueness of class of multidimensional inverse problems, Soviet Math. Dokl. 24 (1981), 244-247.

[BL15] M. Bellassoued and J. Le Rousseau, Carleman estimates for elliptic operators with complex coefficients. Part I: boundary value problems, J. Math. Pures Appl. 104 (2015), 657-728.

[BLR92] C. Bardos, G. Lebeau, and J. Rauch, Sharp sufficient conditions for the observation, control, and stabilization of waves from the boundary, SIAM J. Control Optim. 30 (1992), 10241065.

[BN02] A. Benabdallah and M. G. Naso, Null controllability of a thermoelastic plate, Abstr. Appl. Anal. 7 (2002), 585-599.

[Bur98] Nicolas Burq, Décroissance de l'énergie locale de l'équation des ondes pour le problème extérieur et absence de résonance au voisinage du réel, Acta Math. (1998), 1-29.

[BY12] M. Bellassoued and M. Yamamoto, Carleman estimate with second large parameter for second order hyperbolic operators in a Riemannian manifold and applications in thermoelasticity cases, Appl. Anal. 91 (2012), no. 1, 35-67.

[Cal58] A.-P. Calderón, Uniqueness in the Cauchy problem for partial differential equations., Amer. J. Math. 80 (1958), 16-36.

[Car39] T. Carleman, Sur une problème d'unicité pour les systèmes d'équations aux dérivées partielles à deux variables indépendantes, Ark. Mat. Astr. Fys. 26B (1939), no. 17, 1-9.

[CR14] P. Cornilleau and L. Robbiano, Carleman estimates for the Zaremba boundary condition and stabilization of waves, Amer. J. Math. 136 (2014), 393-444.

[DCFL $\left.{ }^{+} 17\right] \quad$ M. Di Cristo, E. Francini, C.-L. Lin, S. Vessella, and J.-N. Wang, Carleman estimate for second order elliptic equations with Lipschitz leading coefficients and jumps at an interface, J. Math. Pures Appl. (9) 108 (2017), no. 2, 163-206.

[DF88] H. Donnelly and C. Fefferman, Nodal sets of eigenfunctions on Riemannian manifolds, Invent. Math. 93 (1988), 161-183.

[DS15] R. Denk and R. Schnaubelt, A structurally damped plate equation with Dirichlet-Neumann boundary conditions, J. Differential Equations 259 (2015), no. 4, 1323-1353.

[DSF05] David Dos Santos Ferreira, Sharp $L^{p}$ Carleman estimates and unique continuation, Duke Math. J. 129 (2005), no. 3, 503-550.

[DSFKSU09] D. Dos Santos Ferreira, C. E. Kenig, M. Salo, and G. Uhlmann, Limiting carleman weights and anisotropic inverse problems, Invent. Math. 178 (2009), 119-171.

[EI00] M. Eller and V. Isakov, Carleman estimates with two large parameters and applications, Contemporary Math. 268 (2000), 117-136.

[El100] M. Eller, Carleman estimates with a second large parameter, Journal of Mathematical Analysis and Applications 249 (2000), 491-514.

[EMZ15] L. Escauriaza, S. Montaner, and C. Zhang, Observation from measurable sets for parabolic analytic evolutions and applications, J. Math. Pures Appl. 104 (2015), 837-867.

[EMZ17] _ Analyticity of Solutions to Parabolic Evolutions and Applications, SIAM J. Math. Anal. 49 (2017), no. 5, 4064-4092.

[FCZ00] E. Fernández-Cara and E. Zuazua, Null and approximate controllability for weakly blowing up semilinear heat equations, Ann. Inst. H. Poincaré, Analyse non lin. 17 (2000), 583-616.

[FI96] A. Fursikov and O. Yu. Imanuvilov, Controllability of evolution equations, vol. 34, Seoul National University, Korea, 1996, Lecture notes.

[Gao16] P. Gao, The Lebeau-Robbiano inequality for the one-dimensional fourth order elliptic operator and its application, ESAIM Control Optim. Calc. Var. 22 (2016), no. 3, 811-831.

[Hör58] L. Hörmander, On the uniqueness of the Cauchy problem, Math. Scand. 6 (1958), 213-225. 
[Hör63] _ L L L L _ _ _ 1963.

[Hör75] Lars Hörmander, Non-uniqueness for the Cauchy problem, Fourier integral operators and partial differential equations (Colloq. Internat., Univ. Nice, Nice, 1974), Springer, Berlin, 1975, pp. 36-72. Lecture Notes in Math., Vol. 459.

[Hör79] L. Hörmander, The Weyl calculus of pseudo-differential operators, Comm. Pure Appl. Math. 32 (1979), 359-443.

[Hör83] _ Uniqueness theorems for second order elliptic differential equations, Comm. Partial Differential Equations 8 (1983), 21-64.

[Hör85a] _ The Analysis of Linear Partial Differential Operators, vol. IV, Springer-Verlag, 1985.

[Hör85b] _ The Analysis of Linear Partial Differential Operators, vol. III, Springer-Verlag, 1985, Second printing 1994.

[IIY03] O Yu. Imanuvilov, V. Isakov, and M. Yamamoto, An inverse problem for the dynamical Lamé system with two sets of boundary data, Comm. Pure Appl. Math. 56 (2003), 1366-1382.

[IK08] V. Isakov and N. Kim, Carleman estimates with second large parameter and applications to elasticity with residual stress, Applicationes Mathematicae 35 (2008), 447-465.

[Isa98] V. Isakov, Inverse problems for partial differential equations, Springer-Verlag, Berlin, 1998.

[Jaf90] S. Jaffard, Contrôle interne exact des vibrations d'une plaque rectangulaire, Portugal. Math. 47 (1990), no. 4, 423-429.

[JK85] D. Jerison and C. E. Kenig, Unique continuation and absence of positive eigenvalues for Schrödinger operators, Ann. of Math. (2) 121 (1985), no. 3, 463-494, With an appendix by E. M. Stein.

[JL99] D. Jerison and G. Lebeau, Harmonic analysis and partial differential equations (Chicago, IL, 1996), Chicago Lectures in Mathematics, ch. Nodal sets of sums of eigenfunctions, pp. 223239, The University of Chicago Press, Chicago, 1999.

[Kah62] J.-P. Kahane, Pseudo-périodicité et séries de Fourier lacunaires, Ann. Sci. École Norm. Sup. (3) 79 (1962), 93-150.

[KSU07] C. E. Kenig, J. Sjöstrand, and G. Uhlmann, The Calderón problem with partial data, Ann. of Math. 165 (2007), 567-591.

[KT01] H. Koch and D. Tataru, Carleman estimates and unique continuation for second-order elliptic equations with nonsmooth coefficients, Comm. Pure Appl. Math. 54 (2001), no. 3, 339-360.

[KT02] Sharp counterexamples in unique continuation for second order elliptic equations, J. Reine Angew. Math. 542 (2002), 133-146.

[KT05] Herbert Koch and Daniel Tataru, Dispersive estimates for principally normal pseudodifferential operators, Comm. Pure Appl. Math. 58 (2005), 217-284.

[Kub00] M. Kubo, Uniqueness in inverse hyperbolic problems-Carleman estimate for boundary value problems, J. Math. Kyoto Univ. 40 (2000), no. 3, 451-473.

[Le 15] J. Le Rousseau, On Carleman estimates with two large parameters, Indiana Univ. Math. J. 64 (2015), 55-113.

[Léa10] M. Léautaud, Spectral inequalities for non-selfadjoint elliptic operators and application to the null-controllability of parabolic systems, J. Funct. Anal. 258 (2010), 2739-2778.

[Leb96] G. Lebeau, Équation des ondes amorties, Algebraic and geometric methods in mathematical physics (Kaciveli, 1993), Math. Phys. Stud., vol. 19, Kluwer Acad. Publ., Dordrecht, 1996, pp. 73-109.

[Ler88] N. Lerner, Carleman's and subelliptic estimates, Duke Math. J. 56 (1988), 385-394.

[Ler10] Metrics on the Phase Space and Non-Selfadjoint Pseudo-Differential Operators, Pseudo-Differential Operators, Vol. 3, Birkhäuser, Basel, 2010.

[Lin91] F.-H. Lin, Nodal sets of solutions of elliptic and parabolic equations, Comm. Pure Appl. Math. 44 (1991), 287-308. 
[LL12] J. Le Rousseau and G. Lebeau, On Carleman estimates for elliptic and parabolic operators. Applications to unique continuation and control of parabolic equations, ESAIM: Control, Optimisation and Calculus of Variations 18 (2012), 712-747.

[LL13] J. Le Rousseau and N. Lerner, Carleman estimates for anisotropic elliptic operators with jumps at an interface, Anal. PDE 6 (2013), 1601-1648.

[LM68] J.-L. Lions and E. Magenes, Problèmes aux Limites Non Homogènes, vol. 1, Dunod, 1968.

[LR95] G. Lebeau and L. Robbiano, Contrôle exact de l'équation de la chaleur, Comm. Partial Differential Equations 20 (1995), 335-356.

[LR10] J. Le Rousseau and L. Robbiano, Carleman estimate for elliptic operators with coefficents with jumps at an interface in arbitrary dimension and application to the null controllability of linear parabolic equations, Arch. Rational Mech. Anal. 105 (2010), 953-990.

[LR11]_Local and global Carleman estimates for parabolic operators with coefficients with jumps at interfaces, Invent. Math. 183 (2011), 245-336.

[LZ98] G. Lebeau and E. Zuazua, Null-controllability of a system of linear thermoelasticity, Arch. Rational Mech. Anal. 141 (1998), 297-329.

[Mil07] L. Miller, On the cost of fast controls for thermoelastic plates, Asymptot. Anal. 51 (2007), 93-100.

[Nou09] S. Nouira, Polynomial and analytic boundary feedback stabilization of square plate, Bol. Soc. Parana. Mat. (3) 27 (2009), no. 2, 23-34.

[Paz83] A. Pazy, Semigroups of Linear Operators and Applications to Partial Differential Equations, Springer-Verlag, New York, 1983.

[Pli61] A. Pliś, A smooth linear elliptic differential equation without any solution in a sphere, Comm. Pure Appl. Math. 14 (1961), 599-617.

[Rob95] L. Robbiano, Fonction de coût et contrôle des solutions des équations hyperboliques, Asymptotic Anal. 10 (1995), 95-115.

[RTT06] K. Ramdani, T. Takahashi, and M. Tucsnak, Internal stabilization of the plate equation in a square: the continuous and the semi-discretized problems, J. Math. Pures Appl. (9) 85 (2006), no. 1, 17-37.

[Sog89] C. D. Sogge, Oscillatory integrals and unique continuation for second order elliptic differential equations, J. Amer. Math. Soc. 2 (1989), no. 3, 491-515.

[Tat96] D. Tataru, Carleman estimates and unique continuation for solutions to boundary value problems, J. Math. Pures Appl. 75 (1996), 367-408.

[Teb09] L. Tebou, Well-posedness and stability of a hinged plate equation with a localized nonlinear structural damping, Nonlinear Anal. Theory, Methods \& Applications. 71 (2009), no. 12, e2288-e2297.

[Teb12] - Well-posedness and stabilization of an Euler-Bernoulli equation with a localized nonlinear dissipation involving the p-Laplacian, Discrete Contin. Dyn. Syst. Series A 32 (2012), no. 6, 2315-2337.

[Zui83] C. Zuily, Uniqueness and Non Uniqueness in the Cauchy Problem, Birkhauser, Progress in mathematics, 1983.

Jérôme Le Rousseau. Laboratoire Analyse, Géométrie et Applications, CNRS UMR 7539, université Paris-Nord, 93430 Villetaneuse, France, Institut universitaire de France.

E-mail address: jlr@math.univ-paris13.fr

Luc Robbiano. Laboratoire de Mathématiques de Versailles, UVSQ, CNRS, Université Paris-Saclay, 78035 Versaillees, France

E-mail address: luc.robbiano@uvsq.fr 